\title{
VARIATIONS IN CLIMATIC \\ CHARACTERISTICS AS RELATED TO \\ EVAPOTRANSPIRATION IN SOUTH PARK, CENTRAL PARK COUNTY, COLORADO
}

\section{U.S. GEOLOGICAL SURVEY}

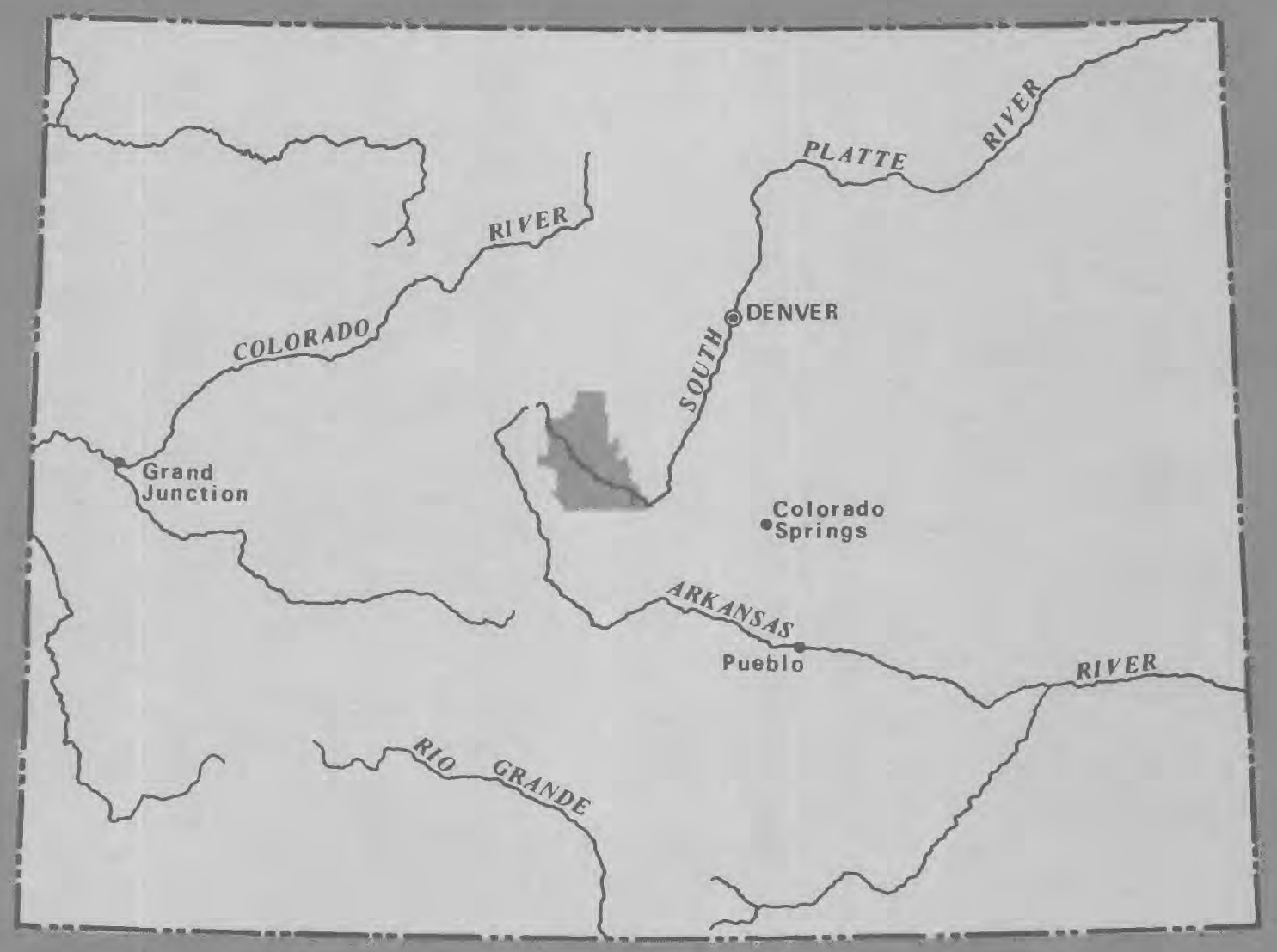

Water-Resources Investigations 80-86

Prepared in cooperation with the City and County of Denver Board of Water Commissioners. 


\begin{tabular}{|c|c|c|}
\hline $\begin{array}{l}\text { REPORT DOCUMENTATION } \\
\text { PAGE }\end{array}$ & 1. REPORT NO. & 3. Recipient's Accession No. \\
\hline \multirow{2}{*}{\multicolumn{2}{|c|}{$\begin{array}{l}\text { 4. Title and subtitle } \\
\text { VARIATIONS IN CLIMATIC CHARACTERISTICS AS RELATED TO EVAPO- } \\
\text { TRANSPIRATION IN SOUTH PARK, CENTRAL PARK COUNTY, COLORADO }\end{array}$}} & \multirow[t]{2}{*}{1981} \\
\hline & & \\
\hline \multicolumn{2}{|l|}{ 7. Author(s) } & 8. Performing Organization Rept. No \\
\hline \multicolumn{2}{|c|}{$\begin{array}{l}\text { U.S. Geological Survey, Water Resources Division } \\
\text { Box 25046, Denver Federal Center, Mail Stop } 415 \\
\text { Lakewood, C0 } 80225\end{array}$} & $\begin{array}{l}\text { 11. Contract(C) or Grant(G) No. } \\
\text { (C) }\end{array}$ \\
\hline \multirow{2}{*}{\multicolumn{2}{|c|}{$\begin{array}{l}\text { 12. Sponsoring Organization Name and Address } \\
\text { U.S. Geological Survey, Water Resources Division } \\
\text { Box 25046, Denver Federal Center, Mail Stop } 415 \\
\text { Lakewood, C0 } 80225\end{array}$}} & $\begin{array}{l}\text { 13. Type of Report \& Period Covered } \\
\text { Final }\end{array}$ \\
\hline & & 14. \\
\hline
\end{tabular}

15. Supplementary Notes

16. Abstract (Limit: 200 words)

Data collected during May through September in 1977, 1978, and 1979 at three stations were analyzed using an analysis of variance technique to determine variations in climatic characteristics in South Park. Knowledge of these climatic characteristics will aid in determining the amount of water that may be transferred from agricultural use in South Park to municipal use in the Denver metropolitan area.

Daily minimum air temperature, daily average air temperature, cumulative wind, daily relative humidity, and daily solar radiaion were statistically different between the three stations at the 1-percent level of significance. Daily maximum air temperature and daily pan evaporation were not significantly different between some stations. Daily precipitation was not significantly different between the three stations.

Estimates of potential evapotranspiration made using the Penman equation were not significantly different between the three stations. The lack of spatial variations in the estimated potential evapotranspiration shows that no one climatic characteristic can be used as an indicator of spatial variation of potential evapotranspiration. Larce variations in solar radiation between the three stations indicate that solar radiation needs to be measured at sites where evapotranspiration is being determined.

17. Document Analysis a. Descriptors

Climatology, Colorado, Evapotranspiration, Statistics, Consumputive use

b. Identifiers/Open-Ended Terms

Analysis of variance, $\mathrm{Cl}$ imatic characteristics, Penman equation, Spatial variation

c. COSATI Field/Group

18. Availability Statement

No restriction on distribution

\begin{tabular}{|r|} 
19. Security Class (This Report) \\
Unclass ified
\end{tabular}

Unclassified 
VARIATIONS IN CLIMATIC CHARACTERISTICS AS

RELATED TO EVAPOTRANSPIRATION IN SOUTH PARK,

CENTRAL PARK COUNTY, COLORADO

By Norman E. Spahr

U.S. GEOLOGICAL SURVEY

Water-Resources Investigations $\mathbf{8 0 - 8 6}$

Prepared in cooperation with the

CITY AND COUNTY OF DENVER,

BOARD OF WATER COMMISSIONERS

Lakewood, Colorado

1981 


\section{UNITED STATES DEPARTMENT OF THE INTERIOR \\ JAMES G. WATT, Secretary GEOLOGICAL SURVEY}

Doyle G. Frederick, Acting Director

For additional information write to:

District Chief

U.S. Geological Survey

Box 25046, Mail Stop 415

Denver Federal Center

Lakewood, CO 80225 
Abstract. .......................

Introduction

Description of South Park

Data collection and conversion

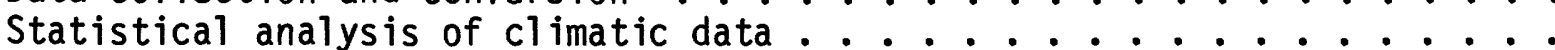

Areal variations in climatic characteristics. .............

Temperature....................

Pan evaporation. ..................

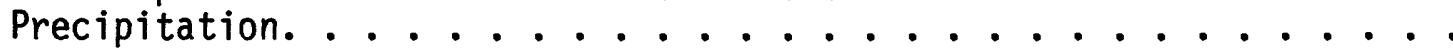

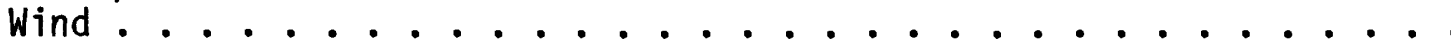

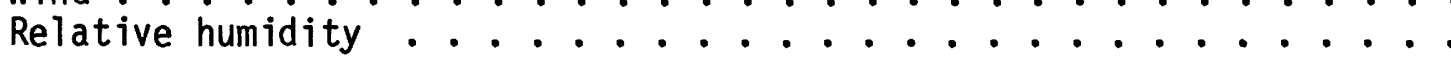

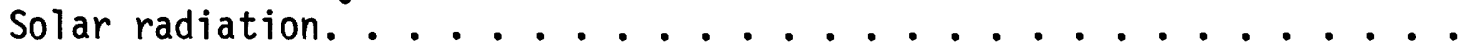

Estimates of potential evapotranspiration . . . . . . . . . . .

Relationship of study data to historic data .............

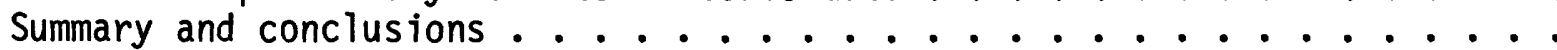

Selected references ...................

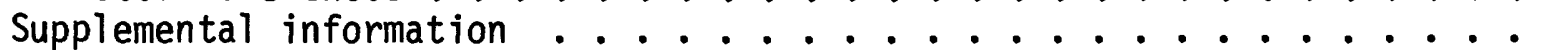

\section{ILLUSTRATIONS}

Figure 1. Map showing location of study area and weather stations. . . .

2-12. Graphs showing:

2. Seven-day mean of daily maximum temperature, Jefferson,

Fairplay, and Antero Reservoir stations, May-

September 1977, 1978, and 1979 .........

3. Seven-day mean of daily minimum temperature, Jefferson,

Fairplay, and Antero Reservoir stations, May-

September 1977, 1978, and 1979 ..........

4. Total May-September precipitation, Hartsel, 1940-64, and Antero Reservoir station, 1962-79........ 15

5. Average May-September air temperature, Antero Reservoir station, 1962-79

6. Seven-day mean of daily pan evaporation, Jefferson,

Fairplay, and Antero Reservoir stations, May-

September 1977, 1978, and 1979 ..........

7. Seven-day total precipitation, Jefferson, Fairplay, and
Antero Reservoir stations, May-September 1977, 1978,

7. Seven-day total precipitation, Jefferson, Fairplay, and
Antero Reservoir stations, May-September 1977, 1978,

and $1979 \ldots \ldots$

8. Seven-day mean of daily cumulative wind, Jefferson,

Fairplay, and Antero Reservoir stations, May-

September 1977, 1978, and 1979 ........... 


\section{CONTENTS}

Figures 2-12. Graphs showing--Continued:

Page

9. Seven-day mean of daily air temperature, Jefferson, Fairplay, and Antero Reservoir stations, May-

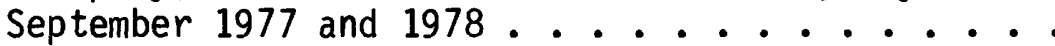

10. Seven-day mean of daily relative humidity, Jefferson, Fairplay, and Antero Reservoir stations, May-September 1977 and 1978 ..........

11. Seven-day mean of vapor pressure, Jefferson, Fairplay, and Antero Reservoir stations, MaySeptember 1977 and $1978 \ldots . . . . . . . .$.

12. Seven-day mean of daily solar radiation, Jefferson, Fairplay, and Antero Reservoir stations, May-

September 1977, 1978, and 1979........

TABLES

Table 1. Descriptions of stations at which climatic data were collected. . . . . . . . .

2. Types of climatic data collected at stations ........

3. Results of analysis of variance and tests of least-significant difference (LSD).

4. Climatic data, Jefferson station, May-September 1977, 1978,

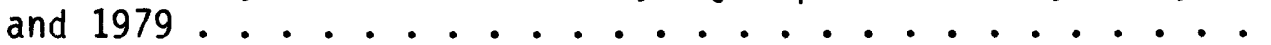

5. Climatic data, Fairplay station, May-September 1977, 1978, and

6. Climatic data, Antero Reservoir station, May-September $1977^{\circ}$

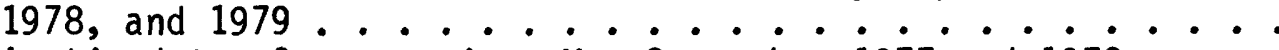

7. Climatic data, Garo station, May-September 1977 and 1978 . . .

Multiply inch-pound unit

inch

mile

square mile

foot

langley

millibar

inches of mercury
By

25.4

1.609

2.590

0.3048

100.

33.8639
To obtain metric unit

millimeter

kilometer

square kilometer meter

joules per square meter pascal

millibars 
VARIATIONS IN CLIMATIC CHARACTERISTICS AS

RELATED TO EVAPOTRANSPIRATION IN SOUTH PARK

CENTRAL PARK COUNTY, COLORADO

By Norman E. Spahr

\section{ABSTRACT}

Data collected during May through September in 1977, 1978, and 1979 at the towns of Jefferson and Fairplay, and at Antero Reservoir were analyzed to determine variations in climatic characteristics in South Park. An analysis of variance technique was used to determine between-station differences in climatic characteristics. Knowledge of these climatic characteristics will aid State and local agencies in determining the amount of water that may be transferred from agricultural use in South Park to municipal use in the Denver metropolitan area.

Results of the analysis of variance of the climatic characteristics can be summarized as follows:

1. Daily maximum temperature was statistically different between the Fairplay and Antero Reservoir stations and between the Jefferson and Antero Reservoir stations at the 1-percent level of significance. Daily maximum air temperature was greater at the Antero Reservoir station than at the Jefferson and Fairplay stations.

2. Daily minimum air temperature was statistically different between the three stations at the 1-percent level of significance. The Antero Reservoir station had the greatest average and the Jefferson. station the lowest average daily minimum air temperature.

3. Daily average air temperature from the hygrothermograph records was statistically different between all stations at the 1-percent level of significance. The Antero Reservoir station had the highest and the Fairplay station the lowest daily average air temperature.

4. Daily pan evaporation was statistically different between the Jefferson and Fairplay stations and between the Jefferson and Antero Reservoir stations at the 1-percent level of significance. The Fairplay and Antero Reservoir stations had greater daily pan evaporation than the Jefferson station.

5. Daily cumulative wind was statistically different between the three stations at the 1-percent level of significance. The cumulative wind was greatest at the Fairplay station and lowest at the Jefferson station. 
6. Daily relative humidity was statistically different between the three stations at the 1-percent level of significance. The Jefferson station had the highest and the Fairplay station the lowest relative humidity.

7. Daily solar radiation was statistically different between the three stations at the 1-percent level of significance. Solar radiation was greatest at the Jefferson station and least at the Antero Reservoir station.

8. Daily precipitation was not significantly different between the three stations.

Estimates of potential evapotranspiration made using the Penman equation were not significantly different between the three stations. The lack of spatial variations in the estimated potential evapotranspiration indicates that no one climatic characteristic can be used as an indicator of spatial variation of potential evapotranspiration. Variations in solar radiation, as much as 101 langleys per day, between the stations at Jefferson, Fairplay, and Antero Reservoir indicate that solar radiation needs to be measured at sites where evapotranspiration is being determined.

With the exception of precipitation during 1977 at the Antero Reservoir station, seasonal precipitation during 1977-78 at all the study stations was less than the 17-year mean seasonal precipitation at Antero Reservoir. In 1979, all stations had greater seasonal precipitation than the 17-year mean seasonal precipitation at Antero Reservoir. During 1977, the seasonal average air temperature at Antero Reservoir was greater than the 17-year mean, during 1978 it was about equal to the 17-year mean, and during 1979 it was slightly below the 17-year mean.

\section{INTRODUCTION}

To provide State and local agencies with data that could be used in determining the amount of water that may be transferred from agricultural use in South Park to municipal use in the Denver metropolitan area, the U.S. Geological Survey, in cooperation with the City and County of Denver. Board of Water Commissioners, conducted a 3-year investigation of climatic characteristics in South Park. Colorado courts have held that the entity purchasing a transfer in water use and point of diversion may obtain only the amount consumptively used by the original right. Therefore, information about the consumptive use is necessary for the reallocation of water resources. Evapotranspiration is one part of the total consumptive use.

The objectives of the study were to determine if spatial variations of climatic characteristics exist and, if so, to determine the magnitude of the variations. Relationships between climatic characteristics and potential evapotranspiration also were investigated to determine if the principal factors controlling the spatial variations of potential evapotranspiration could be identified. 
Appreciation is extended to the station observers, David Paprocki, William Strayer, Denise Moore, Peter Bensen, and Daniel Nyman, for their assistance in collecting the climatic data. Appreciation also is extended to Thomas Kelsay of the National Weather Service for inspecting the station sites and installations to insure that representative data could be collected, and to Brent Troutman of the U.S. Geological Survey for aid in applying analysis of variance to climatic data.

\section{DESCRIPTION OF SOUTH PARK}

South Park is an intermontane valley located in west-central Park County (fig. 1). The park is bounded on the west and north by the Park Range, on the east by the Tarryall Mountains and Puma Hills, and on the south by Black and Thirtynine Mile Mountains. Tributaries of Tarryall Creek drain the northern part of the park and the South Platte River and tributaries drain the remainder of the park. South Park has an area of about 400 square miles. Altitudes within the park range from about 8,800 to about 9,900 feet.

Community and domestic water supply and irrigation of native grasses for use as livestock feed are the principal uses of water in the park. Hay production generally is limited to a single cutting each year because of the short growing season.

\section{DATA COLLECTION AND CONVERSION}

Climatic data suitable for use in this study were available from the National Weather Service only for the precipitation gage at Hartsel operated from 1909 to 1964 and for the precipitation and temperature station at Antero Reservoir operated since 1961. To collect additional data for this study, three stations were established at Jefferson, Fairplay, and Garo, and additional instruments were added to the station at Antero Reservoir. Instruments were installed at the stations during the spring of 1977. Station locations are shown on figure 1 and physical characteristics of the stations are listed in table 1.

Table 1.--Descriptions of stations at which climatic data were collected

\begin{tabular}{|c|c|c|c|c|c|c|}
\hline \multirow{2}{*}{ Station } & \multicolumn{2}{|c|}{ Location 1} & \multirow[b]{2}{*}{$\begin{array}{l}\text { Altitude } \\
\text { (feet) }\end{array}$} & \multirow[b]{2}{*}{$\begin{array}{l}\text { Description } \\
\text { of location }\end{array}$} & \multirow{2}{*}{$\begin{array}{c}\text { Ground cover } \\
\text { at } \\
\text { station }\end{array}$} & \multirow{2}{*}{$\begin{array}{c}\text { Ground cover of } \\
\text { surrounding } \\
\text { area }\end{array}$} \\
\hline & $\begin{array}{l}\text { Latitude } \\
\text { North }\end{array}$ & $\begin{array}{l}\text { Longi tude } \\
\text { West }\end{array}$ & & & & \\
\hline Jefferson-... & $-39^{\circ} 22^{\prime} 32^{\prime \prime}$ & $105^{\circ} 48^{\prime} 12^{\prime \prime}$ & 9,500 & $\begin{array}{l}\text { West edge of } \\
\text { town }\end{array}$ & Grass & $\begin{array}{l}\text { Irrigated ranch- } \\
\text { land. }\end{array}$ \\
\hline Fairplay-.... & $39^{\circ} 13^{\prime} 27^{\prime \prime}$ & $105^{\circ} 58^{\prime} 57^{\prime \prime}$ & 9,870 & $\begin{array}{l}0.2 \text { miles south } \\
\text { of town }\end{array}$ & $\begin{array}{l}\text { Native range- } \\
\text { land }\end{array}$ & Native rangeland. \\
\hline Garo-......... & $39^{\circ} 05^{\prime} 10^{\prime \prime}$ & $105^{\circ} 52^{\prime} 02^{\prime \prime}$ & 9,110 & $\begin{array}{l}2.0 \text { miles south } \\
\text { of town }\end{array}$ & --- do $-\cdots$ & $\begin{array}{l}\text { Irrigated ranch- } \\
\text { land; low ridge } \\
\text { to east. }\end{array}$ \\
\hline $\begin{array}{l}\text { Antero } \\
\text { Reservoir. }\end{array}$ & $38^{\circ} 55^{\prime} 36^{\prime \prime}$ & $105^{\circ} 52^{\prime} 37^{\prime \prime}$ & 8,910 & $\begin{array}{l}0.2 \text { miles south- } \\
\text { east of } \\
\text { reservoir }\end{array}$ & --- do--.- & $\begin{array}{l}\text { Native rangeland; } \\
\text { reservoir to } \\
\text { northwest. }\end{array}$ \\
\hline
\end{tabular}

\footnotetext{
${ }^{1}$ Location shown on figure 1.
} 

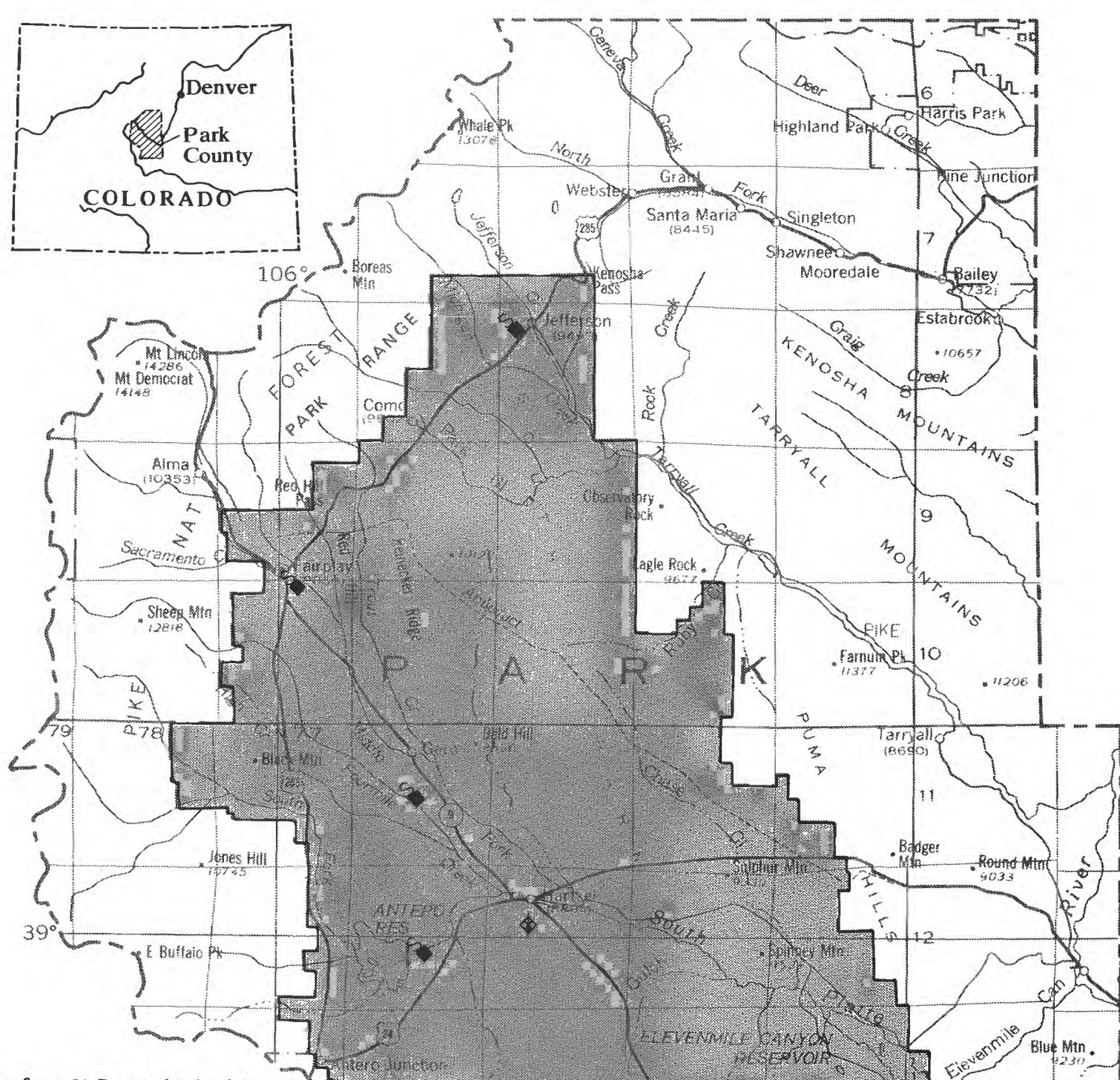

Base from U.S. Geological Survey 1:500 000 State base map, 1969

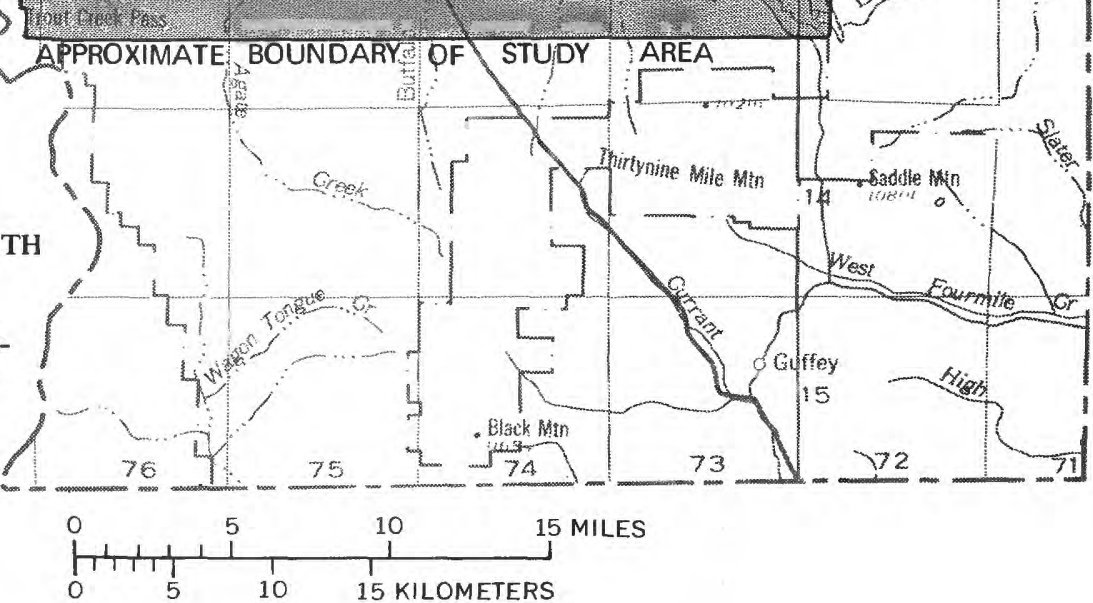

Figure 1.-- Location of study area and weather stations. 
The study originally was to collect data during the 1977 and 1978 growing seasons (May through September) but a third season (1979) of data was collected for some characteristics. Observations were made in the morning at the Jefferson, Garo, and Antero Reservoir stations and in the afternoon at the Fairplay station. All data were collected according to standard procedures of the National Weather Service (1972).

The types of climatic data collected at each station are listed in table 2. Hygrothermograph data were not collected in 1979. Continuous records from the hygrothermographs and radiometers were converted to average daily values by electronically digitizing the areas of the graphical output. With the exception of the daily precipitation data, daily values were combined to determine 7-day (for example, May 1 through May 7) means of each type of data. The combining of daily values to weekly means was done to simplify the data analysis. Daily precipitation values were converted to 7-day total-precipitation values for analysis. Because only a limited amount of data were collected at the Garo station, these data were not used in the statistical analyses. Tabulations of the data collected at the stations are found in the Supplemental Information section (Jefferson station, table 4; Fairplay station, table 5; Antero Reservoir station, table 6; and Garo station, table 7).

\section{STATISTICAL ANALYSIS OF CLIMATIC DATA}

Time graphs for the 7-day means of daily maximum temperature at Jefferson, Fairplay, and Antero Reservoir stations are shown in figure 2 for May-September 1977, 1978, and 1979; and 7-day means of daily minimum temperature at Jefferson, Fairplay, and Antero Reservoir stations are shown in figure 3 for May-September 1977, 1978, and 1979. Time graphs for the other climatic characteristics analyzed (figs. 6-12) are found in the Supplemental Information section. Points are plotted at the median of the 7-day period.

The magnitude of the differences between stations and the week-to-week variations at a single station is apparent in both figures 2 and 3 . To eliminate any effect of the week-to-week variations on the differences between stations, a three-way analysis of variance was used to analyze station differences.

The equation used for the analysis of variance was modified from Graybill (1976) and has the form:

where:

$$
x=\mu+\alpha_{i}+\beta_{j}+\gamma_{k}(j){ }^{+\varepsilon_{i j k^{\prime}}}
$$

$x=$ measured value of variable;

$\mu=$ overal I mean;

$\alpha=f$ ixed effect due to station location;

$\beta=f$ ixed effect due to week of the year:

$\gamma=$ random effect due to year measurements were made; and

$\varepsilon=$ random error term.

Subscripts in equation 1 are standard matrix notations for nested analysis of variance (Graybill, 1976). 


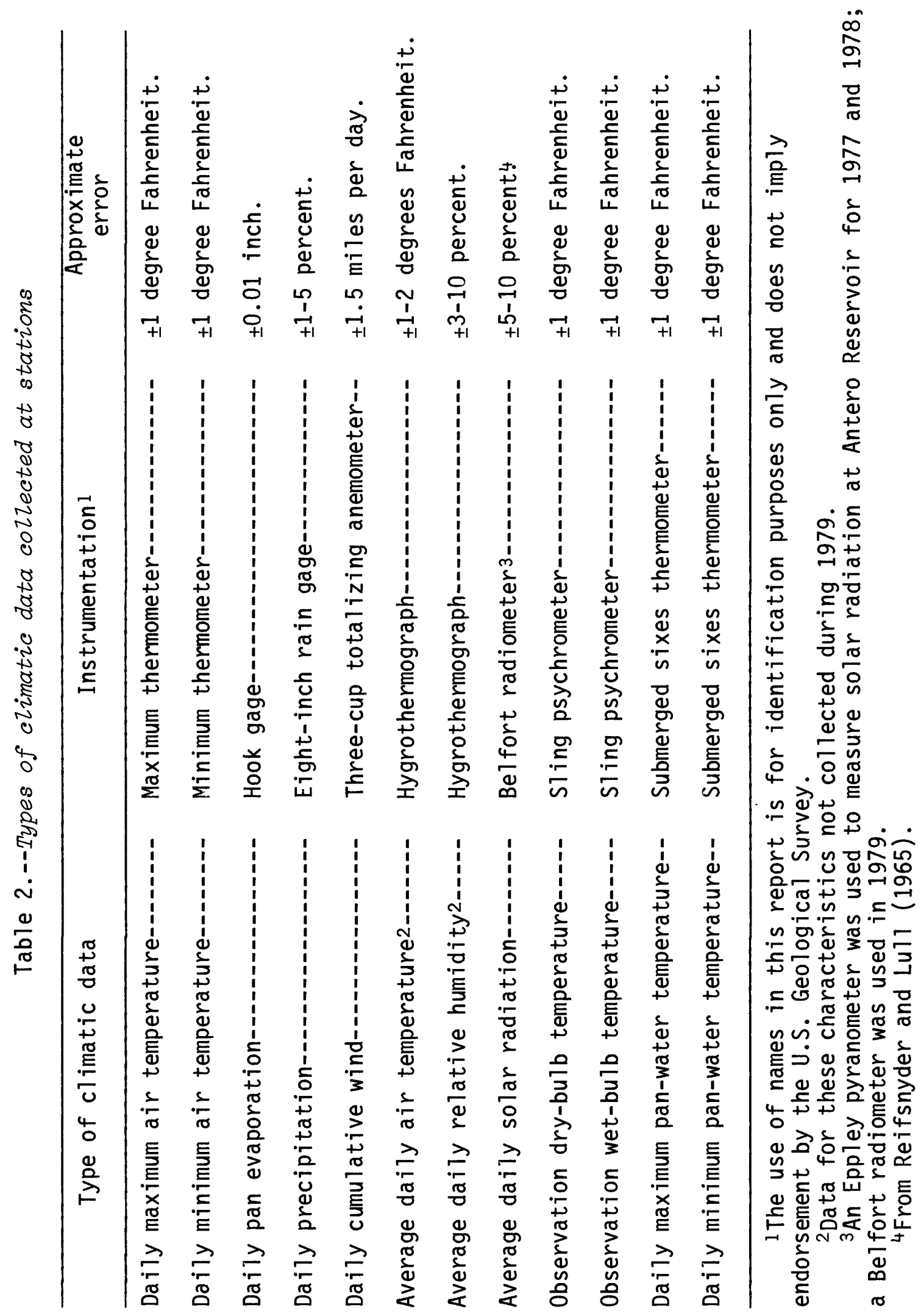




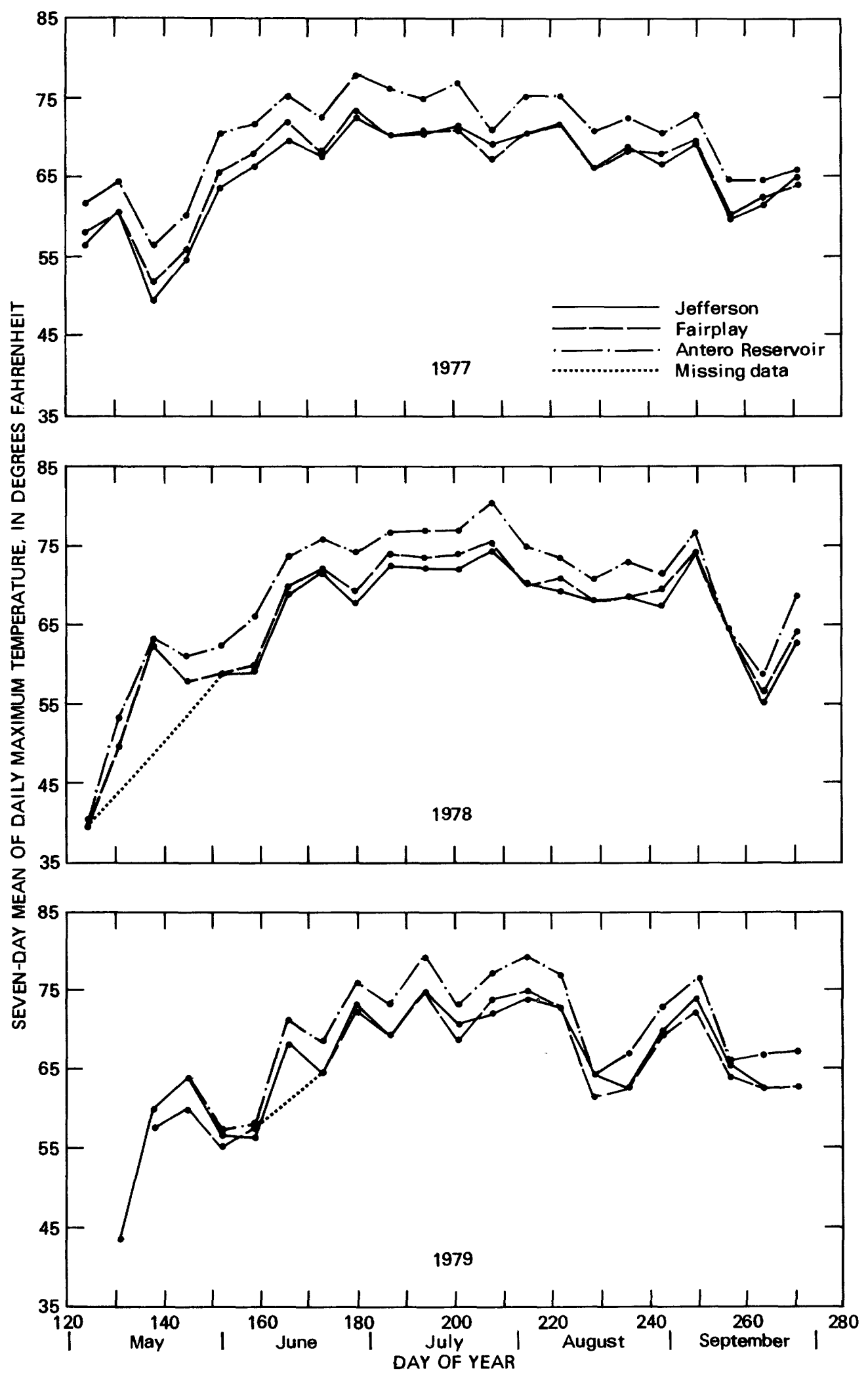

Figure 2.-- Seven-day mean of daily maximum temperature, Jefferson, Fairplay, and Antero Reservoir stations, May-September 1977, 1978, and 1979. 


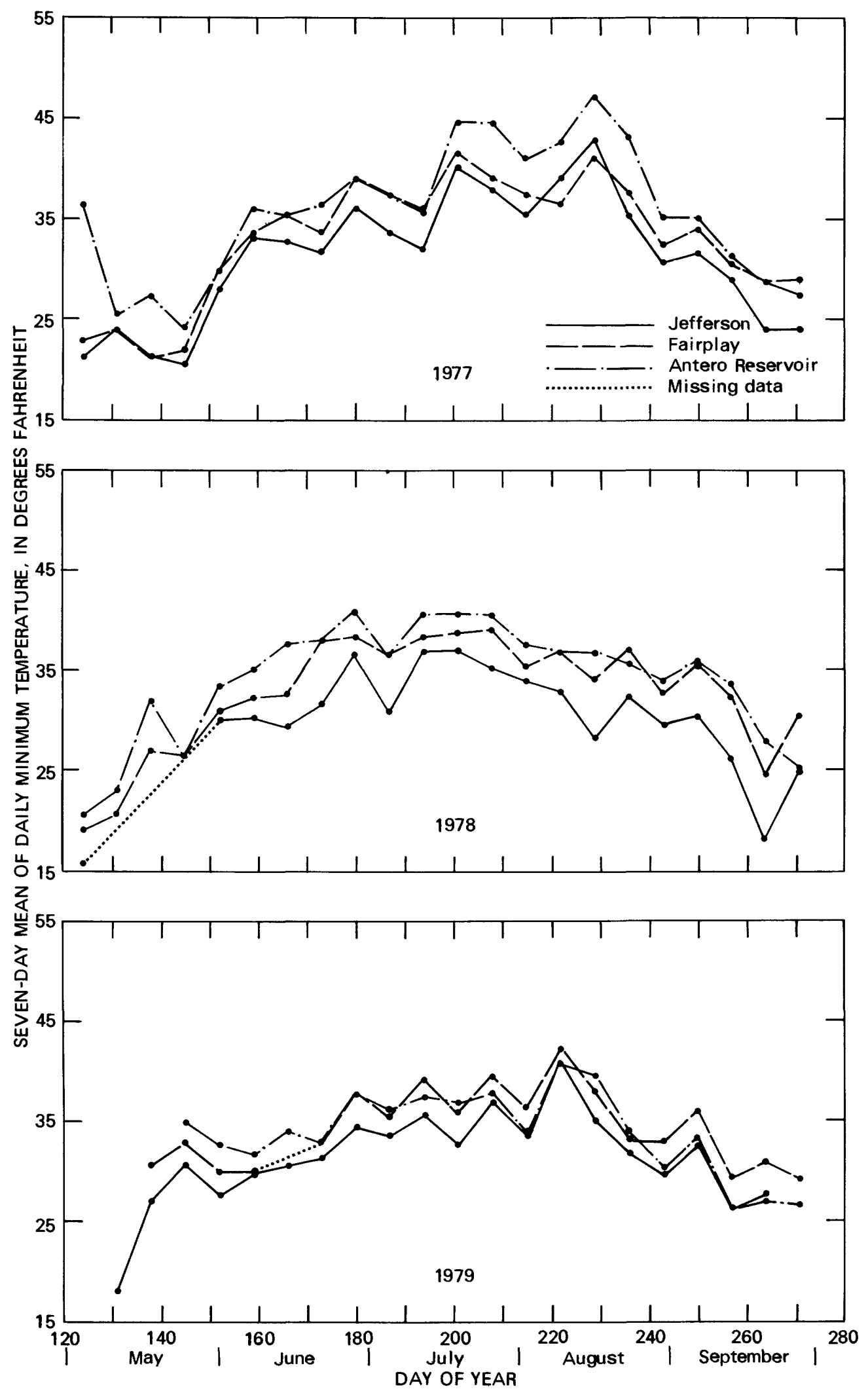

Figure 3.-- Seven-day mean of daily minimum temperature, Jefferson, Fairplay, and Antero Reservoir stations, May-September 1977, 1978, and 1979. 
Equation 1 is known as a mixed three-way analysis of the sources of variation (station location, week of year, year, and error) with nesting of the source year within the source week of year. Equation 1 indicates that any given measured value $(x)$ is a function of the overall mean $(\mu)$ plus the fixed effect due to station location $(\alpha)$ plus the fixed effect due to the week of the year $(\beta)$ plus the random effect due to the year the measurements were made $(\gamma)$ plus a random error term $(\varepsilon)$. By partitioning the total variation, that variation due solely to station location can be analyzed separately. Upon completion of the analysis of variance, an F test (Snedecor and Cochran, 1973) was used to determine if variations between all stations as a group were significant. A least-significantdifference (LSD) test (Snedecor and Cochran, 1973) was then used to analyze the differences between mean values for the climatic characteristics at the different station locations.

The values of the $F$ test are not presented in the report because the analysis between pairs of stations is of more interest. However, in no instance was significance shown for the LSD test when the $F$ test for that characteristic was found not to be significant.

The results of the analyses of variance and the LSD tests are shown in table 3; the first section presents the overall means and the number of weeks analyzed for each climatic characteristic by station, and the second section presents the differences between stations and the corresponding LSD values for each pair of stations. If the difference in overall means is greater than the adjacent LSD value, it can be assumed that the difference in overall means is significant at the 1 -percent level of the LSD test.

The LSD values and the differences in means are estimates of population statistics of the various climatic characteristics and, as such, are based upon numerous point observations. Therefore, it is entirely possible that a particular LSD value may be less than the given instrumentation error because the error is describing a point observation and the LSD value is describing many observations.

\section{AREAL VARIATIONS IN CLIMATIC CHARACTERISTICS}

Using table 3 as a guide, the differences in climatic characteristics between stations can be described. In the following sections, the term "significant" refers to the 1-percent level of significance by the LSD test.

\section{Temperature}

Daily maximum air temperatures were significantly different between the Fairplay and Antero Reservoir stations and between the Jefferson and Antero Reservoir stations but were not significantly different between the Jefferson and Fairplay stations. Both daily minimum air temperatures and average daily air temperatures were significantly different between all three stations. Based upon the hygrothermograph data, daily average air temperatures are related to altitude, with the coldest temperature occurring at Fairplay (altitude, 9,870 feet) and the warmest temperature occurring at Antero Reservoir (altitude, 8,910 feet). 


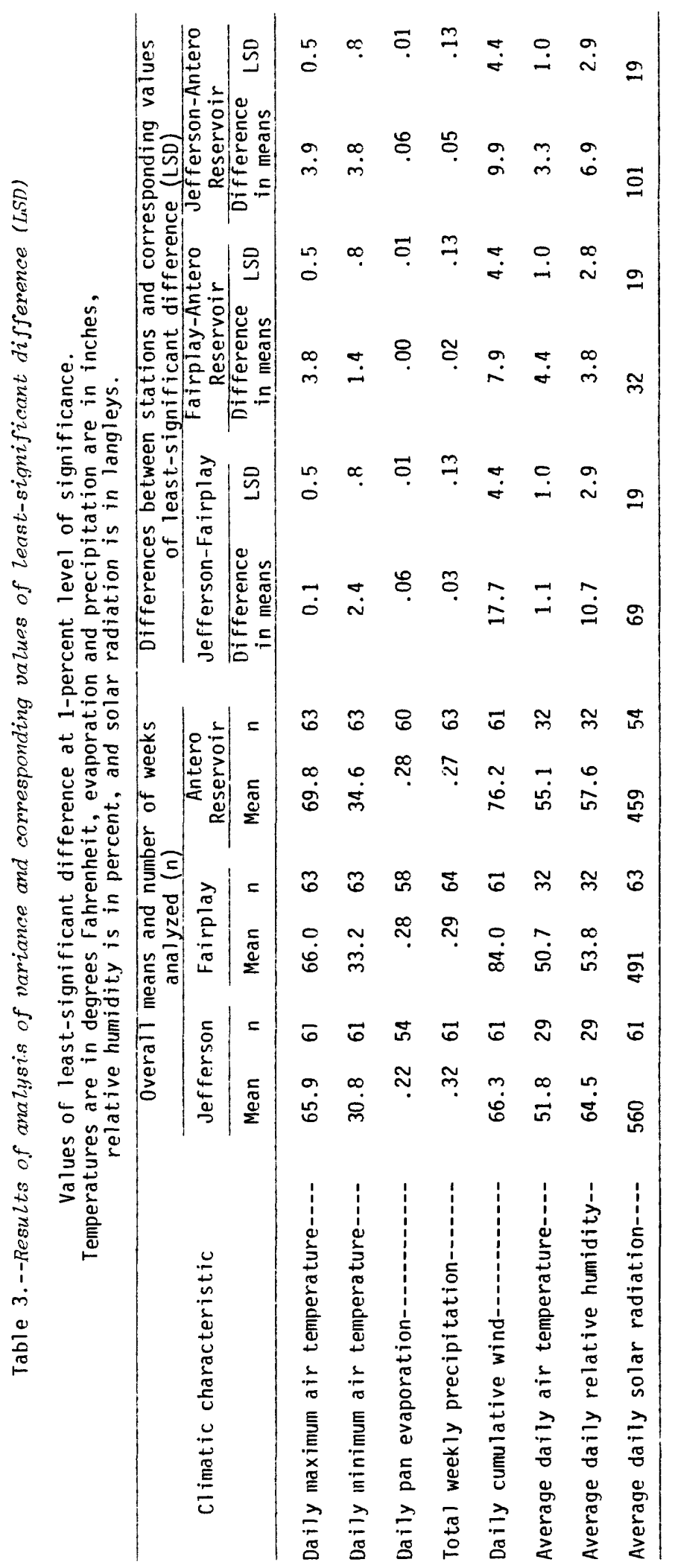


Using the average of daily maximum and daily minimum temperature, the Jefferson station has a lower temperature than the Fairplay station. This apparent contradiction occurs because the hygrothermograph data is an integrated value whereas the average of maximum and minimum temperature is based upon two point values. The closeness of the maximum temperatures between Fairplay and Jefferson also should be noted. More data need to be collected to better define the temperature regime between these two stations.

\section{Pan Evaporation}

Daily pan evaporation was significantly different between the Jefferson and Fairplay stations and between the Jefferson and Antero Reservoir stations but was not significantly different between the Fairplay and Antero Reservoir stations.

\section{Precipitation}

Total weekly precipitation did not differ significantly between stations. The lack of significant differences does not necessarily mean that every storm distributed precipitation uniformly throughout the park. However, the lack of significant differences does indicate that, on the average, the total weekly precipitation resulting from all storms was about the same at all the stations.

\section{Wind}

Daily cumulative wind was significantly different between all stations. The degree of significant difference was the greatest of all the climatic characteristics. This indicates that daily cumulative wind may be a significant individual climatic characteristic affecting the total climatic conditions at all three stations and likely throughout the South Park area.

\section{Relative Humidity}

Average daily relative humidity was significantly different between all stations. However, because of the character of the hygrothermograph instrumentation, the 3.8-percent difference between the overall means of the Fairplay and Antero Reservoir stations may be within instrument accuracy. It should be noted that only two seasons of relative humidity data (1977 and 1978) were collected.

\section{Solar Radiation}

Average daily solar radiation was significantly different between all stations. The difference of 101 langleys per day between the overall means of the Jefferson and Antero Reservoir stations appears to be unusually large, and may be due to the variability in cloud cover as convective storms form and move over the park. The difference in instrumentation also may have an affect on this variability. All 
radiometers were checked with an independent instrument but the Eppley ${ }^{1}$ pyranometer used at the Antero Reservoir station is much more responsive than the mechanical radiometers used at the other stations. In 1979 mechanical radiometers were used at all three stations. The same type of variation was present; therefore, the instrumentation difference in 1977 and 1978 may not play a major role.

\section{ESTIMATES OF POTENTIAL EVAPOTRANSPIRATION}

To show how the climatic characteristics are incorporated into an index value of evapotranspiration and to show the areal variation of the index, potential evapotranspiration values were determined for the Jefferson. Fairplay, and Antero Reservoir stations for 1977 and 1978. Because hygrothermograph data were not collected in 1979, estimates of potential evapotranspiration are not given for the 1979 season.

Potential evapotranspiration can be estimated using an equation derived by Penman (1948). This equation is a combination of the energy budget and the vapor-transport theories of evaporation, and represents potential water use from a well-watered, actively growing, short grass crop.

One form of the equation (American Society of Civil Engineers, 1973) is:

$$
\varepsilon=\frac{\Delta}{\Delta+\gamma}\left(R_{n}+G\right)+\frac{\gamma}{\Delta+\gamma} 15.36\left(1.0+.01 U_{2}\right)\left(e_{o}-e\right) \text {. }
$$

where:

$\varepsilon=$ daily potential evapotranspiration, in langleys per day:

$\Delta=$ slope of saturation vapor pressure curve, in millibars per degrees

Celsius:

$\gamma=$ psychrometric constant, in millibars per degrees Celsius;

$R_{n}=$ net radiation, in langleys per day;

G=heat flux density from ground, in langleys per day;

$\mathrm{U}_{2}=$ horizontal wind speed at 2 meters, in miles per day:

$\mathrm{e}_{\mathrm{o}}=$ saturation vapor pressure at average air temperature, in millibars; and

e=vapor pressure at average dew-po int temperature, in mill ibars.

The slope of the vapor pressure curve $(\Delta)$, the psychrometric constant $(\gamma)$, and the saturation vapor pressure $\left(\mathrm{e}_{\mathrm{o}}\right)$ were calculated using equations by Bosen and Brunt, as presented in American Society of Civil Engineers (1973, p. 123). The values of $\gamma$ are based upon air pressure or defined by the standard pressurealtitude curve. The values of $e_{0}$ and $e$ were based upon hygrothermograph data.

1 The use of the brand name in this report is for identification purposes only and does not imply endorsement by the U.S. Geological Survey. 
In applying this equation to the South Park data, the ground-heat flux term was assumed negligible. The ground-heat flux is a minor contributor to the equation when compared to other heat terms; therefore, neglecting it does not seriously affect results.

Because of the lack of longwave-radiation data, net radiation was estimated from incoming solar radiation by use of a regression equation presented by the American Society of Civil Engineers (1973). The equation is $R_{n}=0.61 R_{s}-24$, where $R_{S}$ is solar radiation in langleys per day. Percentage errors for the equation were not given, but errors in the final potential evapotranspiration values will result from the net radiation estimates. The Penman (1948) formula yields daily estimates which were combined to find weekly mean values of potential evapotranspiration for the Jefferson, Fairplay, and Antero Reservoir stations. The analysis of variance technique was then used to investigate between-station variances.

The overall mean value of daily potential evapotranspiration was found to be 0.16 inch at Jefferson, 0.17 inch at Fairplay, and 0.17 inch at Antero Reservoir. Using the LSD test, a difference of greater than 0.01 inch between stations is necessary for the overall means to be statistically significant at the 1-percent level. Because the maximum difference between any two stations was only 0.01 inch, the estimated potential evapotranspiration was not significantly different between the Jefferson, Fairplay, and Antero Reservoir stations. These estimates of daily potential evapotranspiration are 0.03 inch less than values reported by Kruse and Haise (1974) for a site near Garo for the 1967-69 growing seasons. This small difference may be attributed to normal variation of climatic factors, differences in instrumentation, or differences in applying the Penman equation to measured climatic data.

The Penman equation estimates potential evapotranspiration. This can be thought of as an index. Actual evapotranspiration may be significantly different from the potential values. The derivation of actual evapotranspiration from potential evapotranspiration involves locally calibrating the potential estimates to measured evapotranspiration for the crop in question. The values given and conclusions drawn from these values should not be construed to apply to actual evapotranspiration in the South Park area.

The Penman equation is not the only equation available to determine potential evapotranspiration. It was chosen because most of the climatic characteristics known to affect evapotranspiration are included in the equation. 


\section{RELATIONSHIP OF STUDY DATA TO HISTORIC DATA}

Although the data collected during this study indicate that there is no significant difference in daily pan evaporation between the Fairplay and Antero Reservoir stations, data collected in previous studies indicate that there may be a significant difference in daily pan evaporation between Fairplay, Garo, and Antero Reservoir. According to Ficke and others (1977), pan evaporation at the Antero Reservoir station averaged 0.24 inch per day during May through September 1967-70, while pan evaporation at a site near Garo averaged 0.18 inch per day for the same period according to Kruse and Haise (1974). The apparent differences in daily pan evaporation between Fairplay, Garo, and Antero Reservoir could not be verified during 1977-78 because of insufficient data from the Garo station. Part of this difference could be attributed to non-uniform operation of the evaporation pans. Hansen and others (1978) mapped a slight increase in annual evaporation from Antero Reservoir towards Fairplay but the amou, it of site-specific data available for this area was limited at the time the map was developed.

The relationship between seasonal (May-September) precipitation measured at Hartsel from 1940 through 1964, seasonal precipitation measured at the Antero Reservoir station from 1962 through 1976, and seasonal precipitation measured for this study at the Jefferson, Fairplay, and Antero Reservoir stations from 1977 through 1979 is shown in figure 4. The data for this study at Antero Reservoir are plotted separately from data reported by the U.S. Weather Bureau (1940-79) because the Weather Bureau data reported during 1977 through 1979 covers minor periods not analyzed by analyses of variance.

Mean seasonal precipitation at the Antero Reservoir station has been less than the normal seasonal precipitation (1931-60) at Hartsel. In addition, with the exception of precipitation during 1977 at the Antero Reservoir station, seasonal precipitation during 1977 and 1978 at all stations was less than the 17-year mean seasonal precipitation at Antero Reservoir. In 1979 all stations had greater seasonal precipitation than the 17-year mean seasonal precipitation at Antero Reservoir. Hansen and others (1978) show a mean April-September precipitation value of less than 10 inches for the Antero Reservoir area and 12 to 14 inches for the Jefferson-Fairplay area. The U.S. Weather Bureau (1964) map of normal MaySeptember precipitation shows a value of less than 8 inches for Antero Reservoir, approximately 8 inches for Fairplay, and approximately 9 inches for Jefferson. Therefore, it appears that precipitation during 1977 and 1978 was less than the normal precipitation, and during 1979 might have been slightly above the precipitation normally occurring during the growing season in the area.

Air-temperature data also have been collected at the Antero Reservoir station since 1962 (fig. 5). The average (arithmetic mean of maximum and minimum temperatures) seasonal (May-September) air temperature at the Antero Reservoir station for 1962-79 was 51.2 ${ }^{\circ}$ Fahrenheit. During 1977, the seasonal air temperature was greater than this long-term average, while during 1978 it was about equal to it. Seasonal air temperature during 1979 was slightly below this longterm average. All data shown in figure 5 are from the U.S. Weather Bureau (1940-79). 


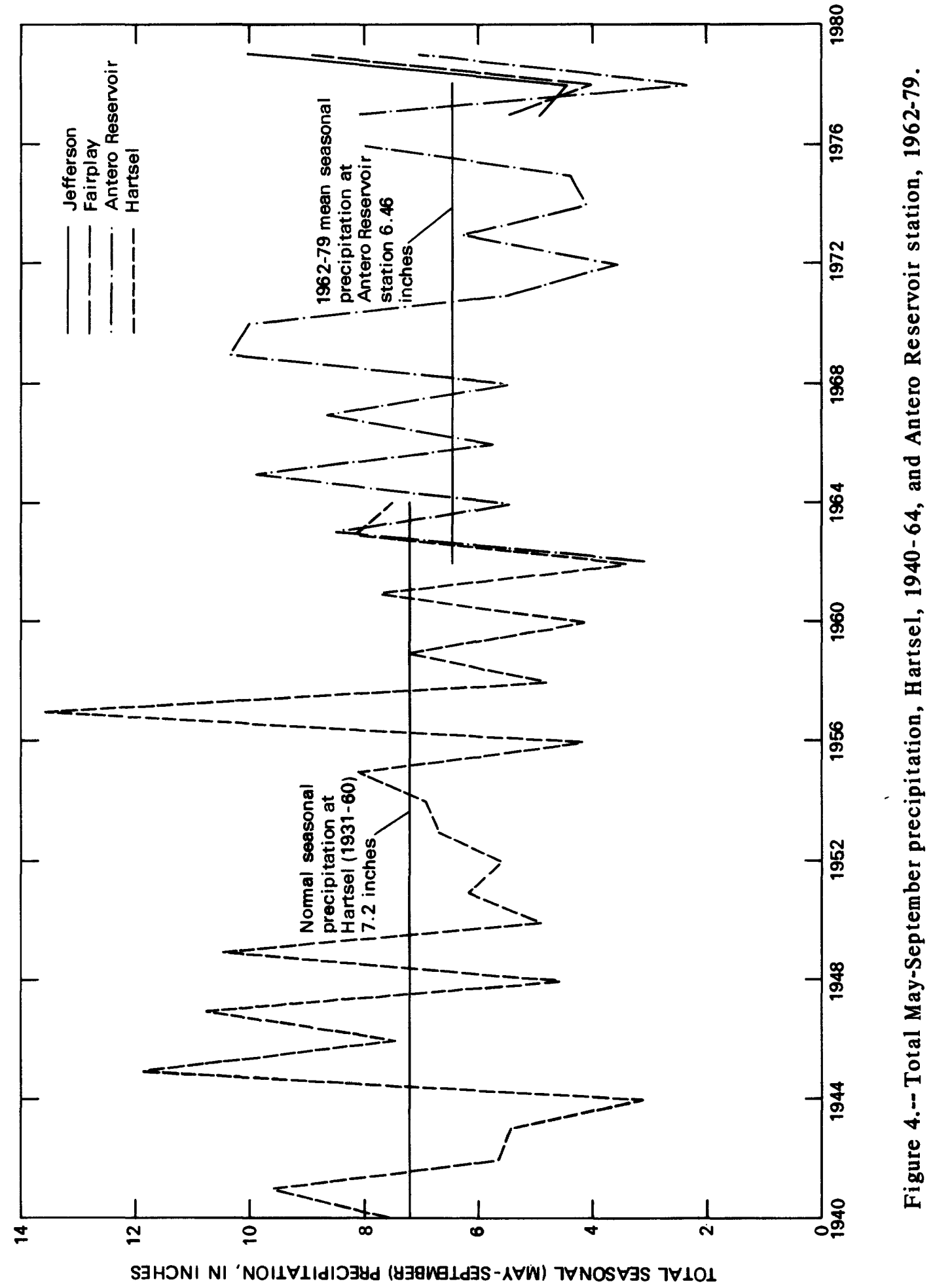




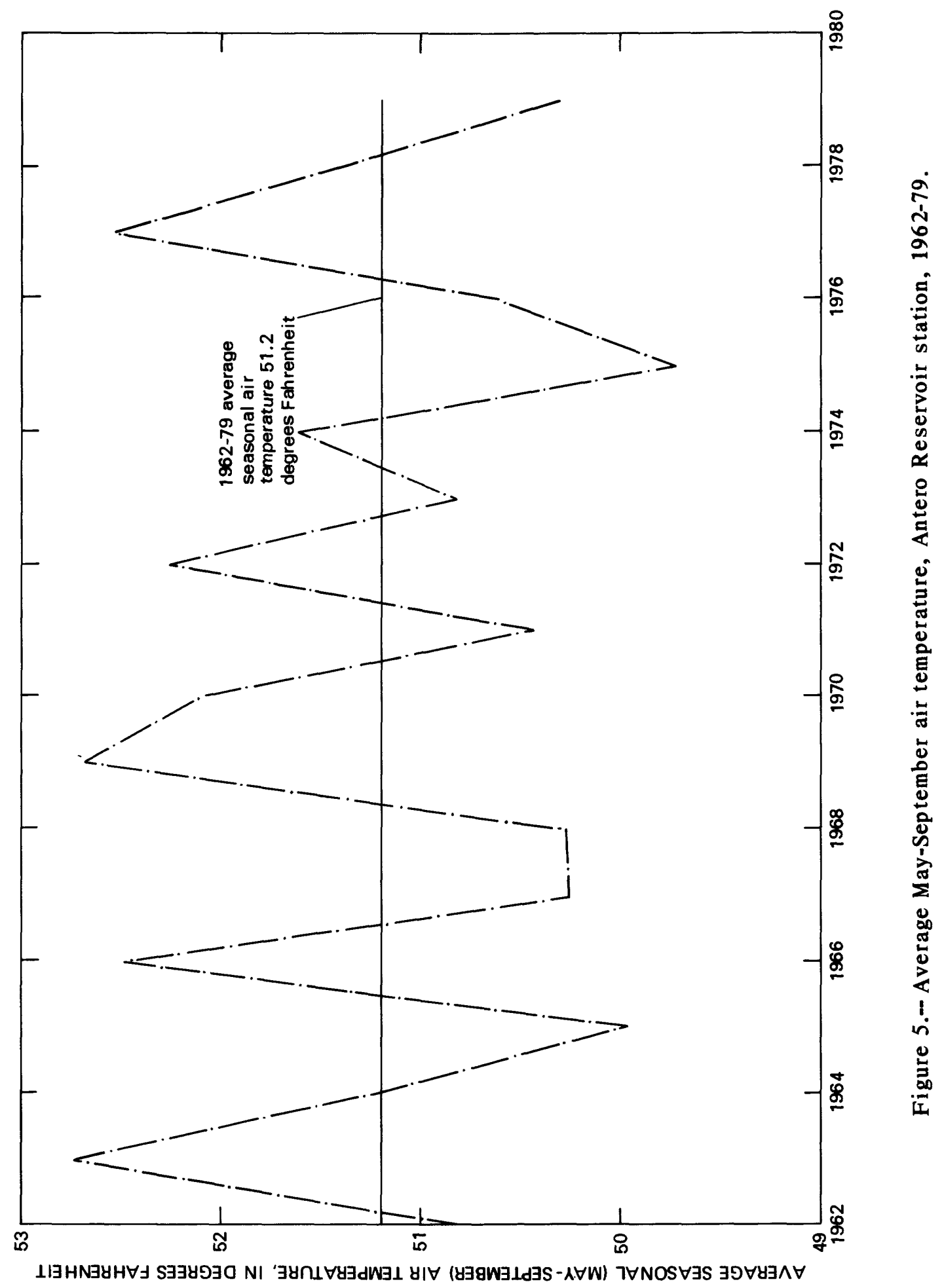




\section{SUMMARY AND CONCLUSIONS}

Maximum and minimum air temperature, daily pan evaporation, daily precipitation, and daily solar radiation were measured at Jefferson, Fairplay, Garo, and Antero Reservoir stations during the growing seasons (May-September) of 1977 , 1978, and 1979. Mean daily air temperature and daily relative humidity were only measured in 1977 and 1978 at these stations. Variations in the climatic characteristics between the Jefferson, Fairplay, and Antero Reservoir stations were analyzed using analysis of variance and a 1-percent least-significant-difference test; insufficient data was obtained at the Garo site for statistical analyses. The analysis can be summarized as follows:

1. Daily maximum air temperature did not differ between the Jefferson and Fairplay stations.

2. Daily pan evaporation was not different between the Fairplay and Antero Reservoir stations.

3. Precipitation did not differ between any of the stations.

4. All other climatic characteristics varied between the Jefferson, Fairplay, and Antero Reservoir stations.

When the study data was compared to historical precipitation data at Hartsel and Antero Reservoir, it was found that the 1977-78 growing seasons were drier than the long-term mean for the area, and that 1979 was wetter than the longterm mean for the area. Also, 1977 and 1978 were slightly warmer and 1979 slightly cooler than the 17-year average seasonal temperature at Antero Reservoir.

Estimated potential evapotranspiration was calculated using the Penman (1948) equation for the Jefferson. Fairplay, and Antero Reservoir stations. The estimates of potential evapotranspiration did not differ significantly between any of the stations. Data for the climatic characteristics--wind, solar radiation, relative humidity, and air temperature--are necessary for use of the Penman equation. Each of these characteristics varied significantly between the Jefferson, Fairplay, and Antero Reservoir stations. When incorporated into the Penman equation, the combined effect of the individual variations in the climatic characteristics was reduced, yielding statistically similar estimates of potential evapotranspiration. Therefore, no single climatic characteristic may be used to indicate spatial variations of potential evapotranspiration in the South Park area. The variations in solar radiation between the Jefferson, Fairplay, and Antero Reservoir stations indicate that solar radiation needs to be measured at any site dealing with evapotranspiration in the South Park area. 


\section{SELECTED REFERENCES}

American Society of Civil Engineers, 1973, Consumptive use of water and irrigation water requirements: New York, American Society of Civil Engineers, 215 p.

Ficke, J. F., Adams, D. B., and Danielson, T. W., 1977, Evaporation from seven reservoirs in the Denver water-supply system, central Colorado: U.S. Geological Survey Water-Resources Investigations 76-114, $170 \mathrm{p}$.

Graybill, F. A.. 1976, Theory and application of the linear model: North Scituate, Mass., Duxbury Press, 704 p.

Hansen, W. R., Chronic, John, and Matelock, John, 1978, Climatography of the Front Range Urban Corridor and vicinity, Colorado: U.S. Geological Survey Professional Paper 1019, $59 \mathrm{p}$.

Kohler, M. A., Nordensen, T. J., and Baker, D. R., 1959, Evaporation maps for the United States: U.S. Weather Bureau Technical Paper 37, $13 \mathrm{p}$.

Kruse, E. G.. and Haise, H. R., 1974, Water use by native grasses in high altitude Colorado meadows: U.S. Department of Agriculture Research Service Paper ARS-W-6, $59 \mathrm{p}$.

National Weather Service, 1972, National Weather Service observing handbook No. 2: Washington, D.C.. U.S. Government Printing Office, $77 \mathrm{p}$.

Penman, H. L., 1948, Natural evaporation from open water, bare soil and grasses: London, England, Proceedings of the Royal Society, Ser. A, v. $193, p .120$.

Reifsnyder, W. E., and Lull, H. W., 1965, Radiant energy in relation to forests: U.S. Department of Agriculture, Forest Service Technical Bulletin 1344, $111 \mathrm{p}$.

Snedecor, G. W., and Cochran, W. G., 1973, Statistical methods: Ames, lowa State University Press, $593 \mathrm{p}$.

U.S. Weather Bureau, 1964, Map of normal May-September precipitation for Colorado: Washington, D.C., U.S. Department of Commerce, scale 1:500,000. 1940-79, Climatological data, Colorado section, annual summaries 1940 through 1979: Washington, D.C., U.S. Government Printing Office. 
SUPPLEMENTAL INFORMATION 


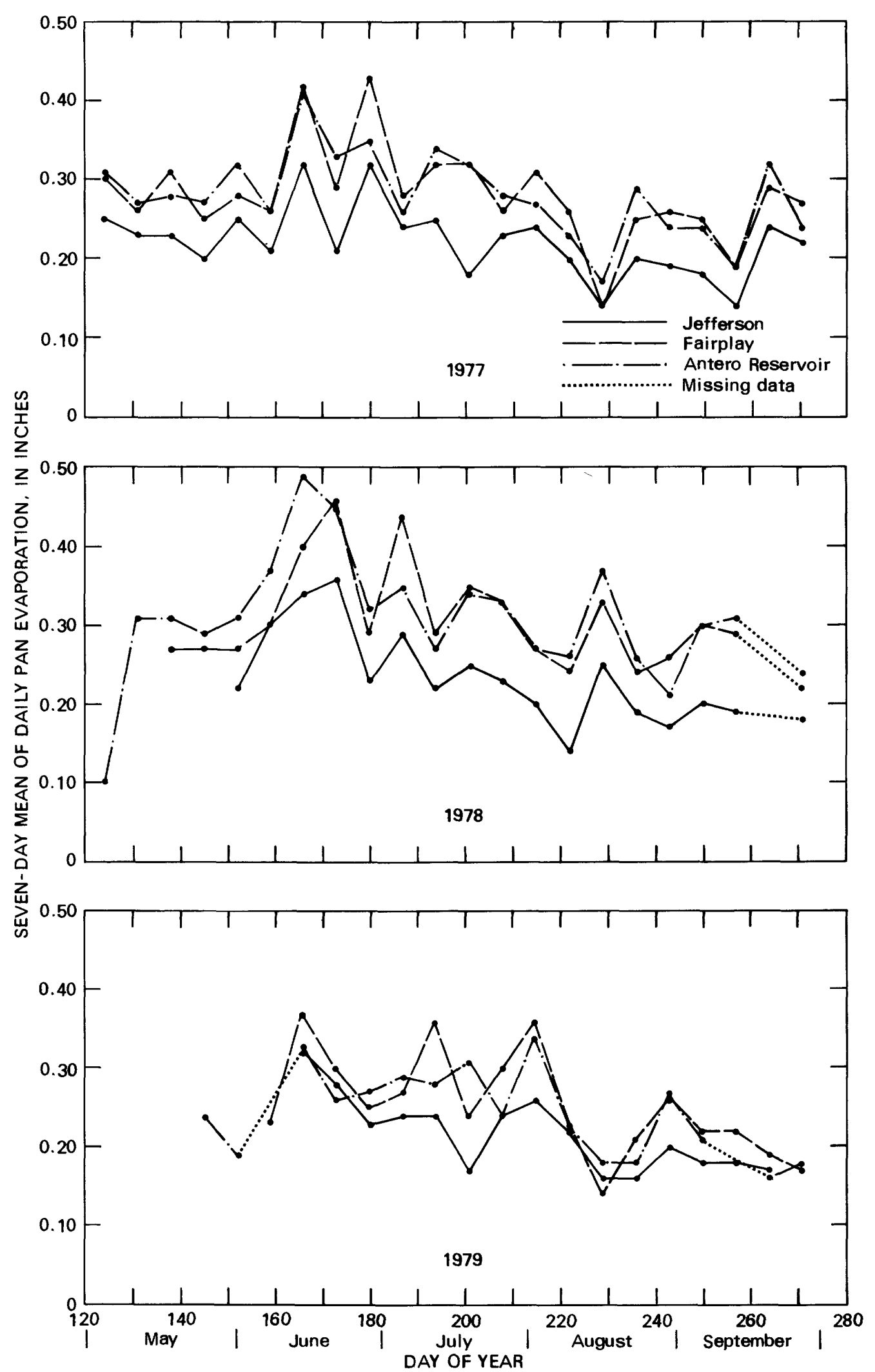

Figure 6.-- Seven-day mean of daily pan evaporation, Jefferson, Fairplay, and Antero Reservoir stations, May-September 1977, 1978, and 1979. 

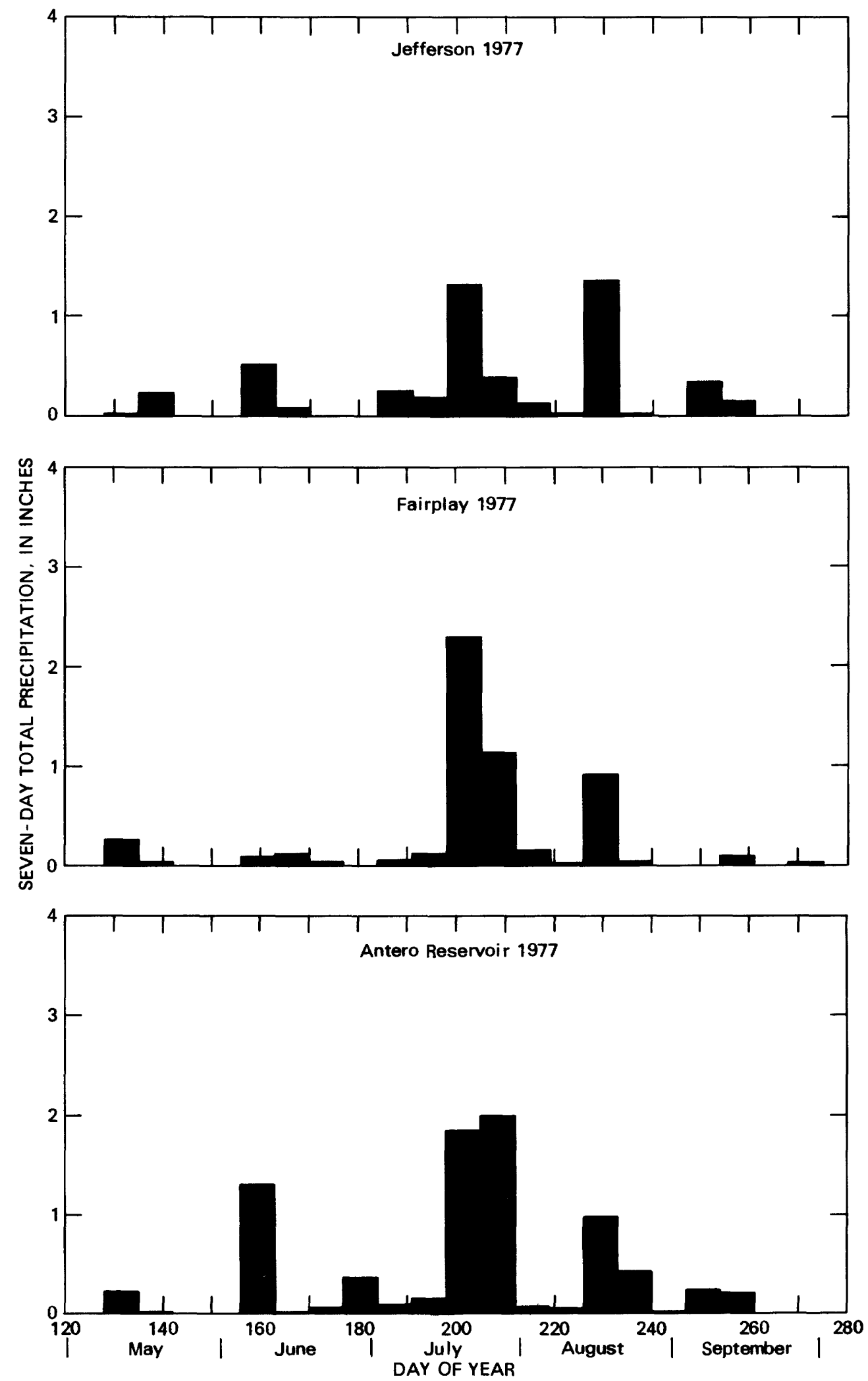

Figure 7.-- Seven-day total precipitation, Jefferson, Fairplay, and Antero Reservoir station s, May-September 1977, 1978, and 1979. 

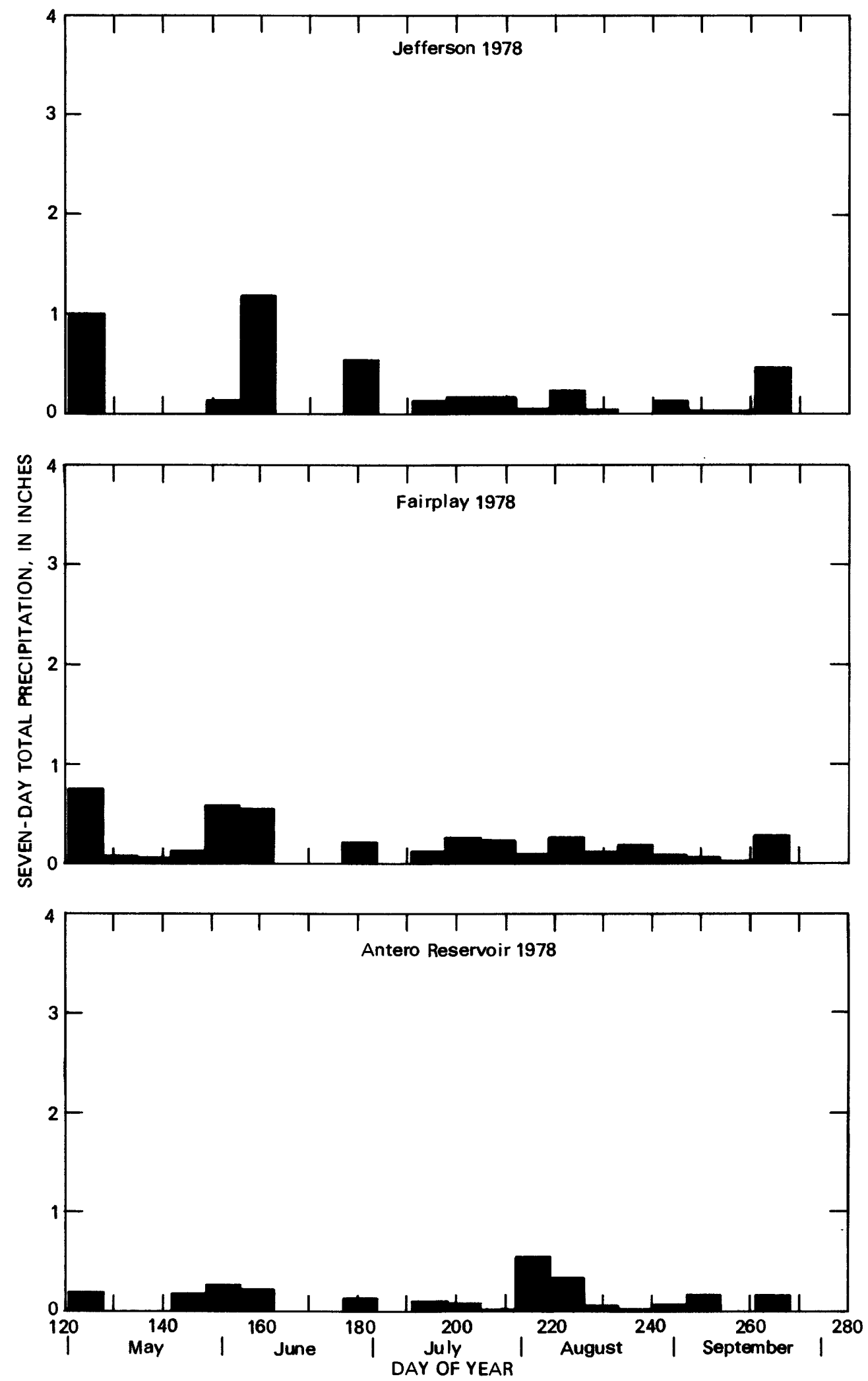

Figure 7.-- Seven-day total precipitation, Jefferson, Fairplay, and An tero Reservoir stations, May-September 1977, 1978, and 1979--Continued. 

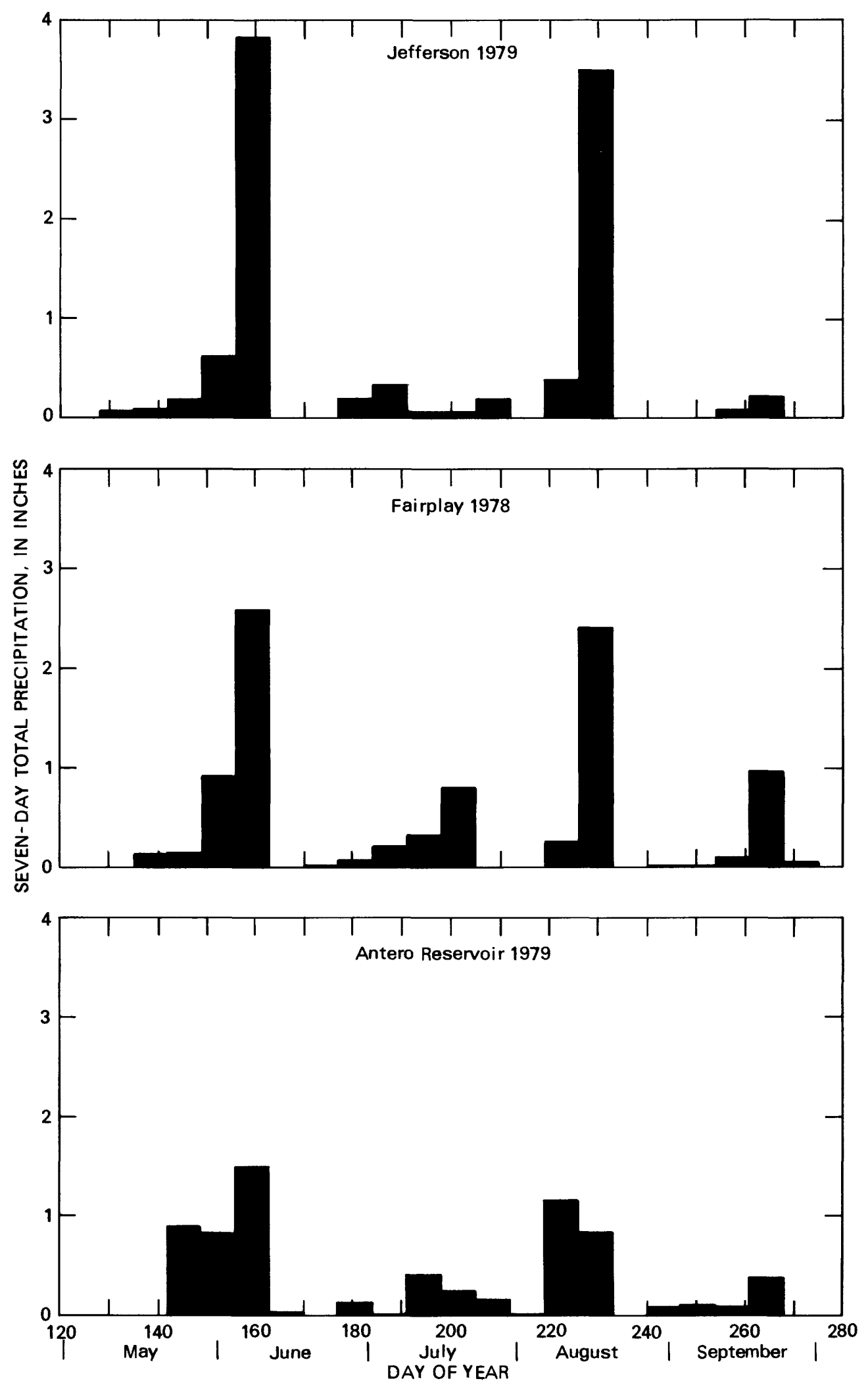

Figure 7.-- Seven-day total precipitation, Jefferson, Fairplay, and Antero Reservoir stations, May-September 1977, 1978, and 1979--Continued. 


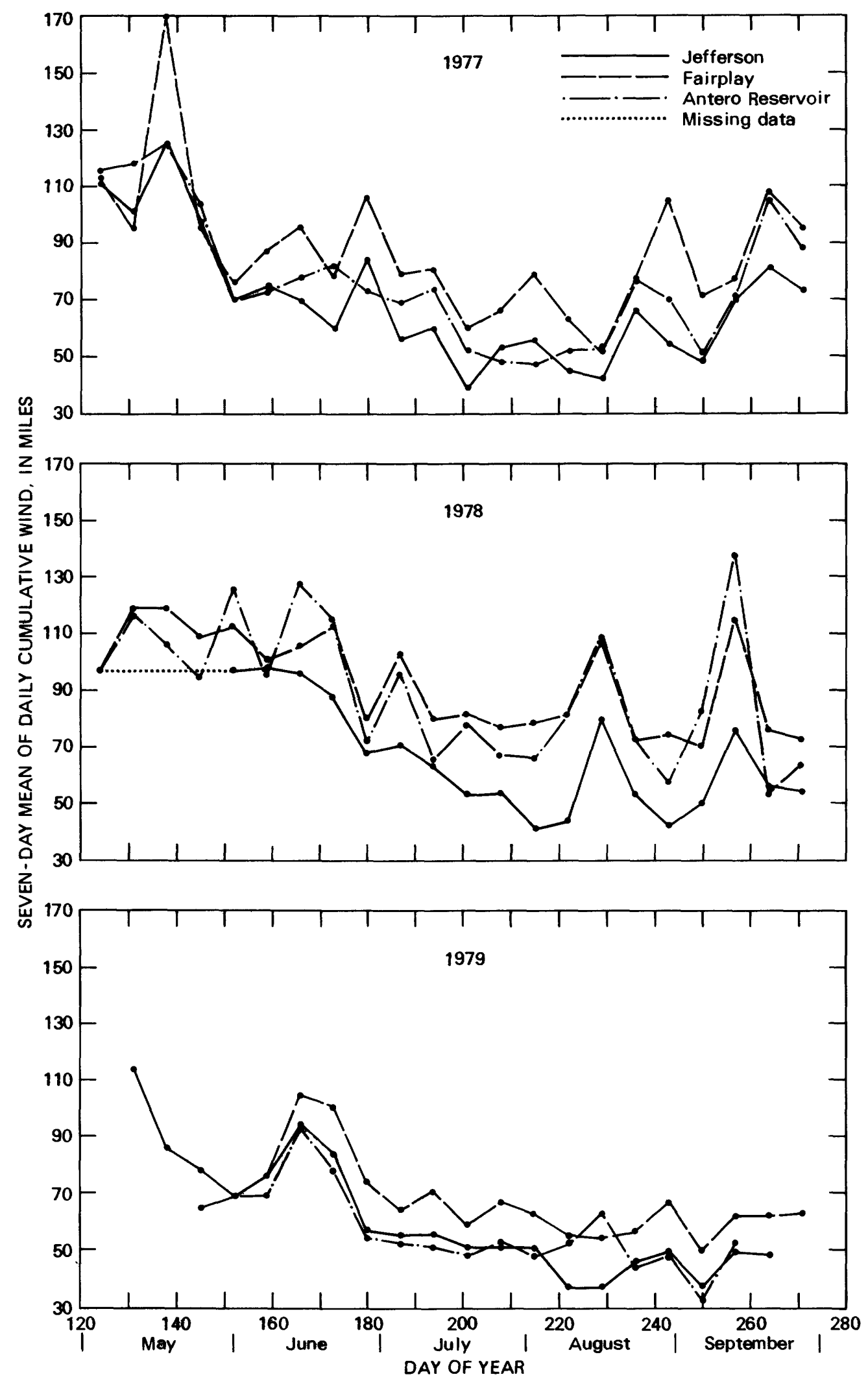

Figure 8.-- Seven-day mean of daily cumulative wind, Jefferson, Fairplay, and Antero Reservoir stations, May-September 1977, 1978 and 1979. 


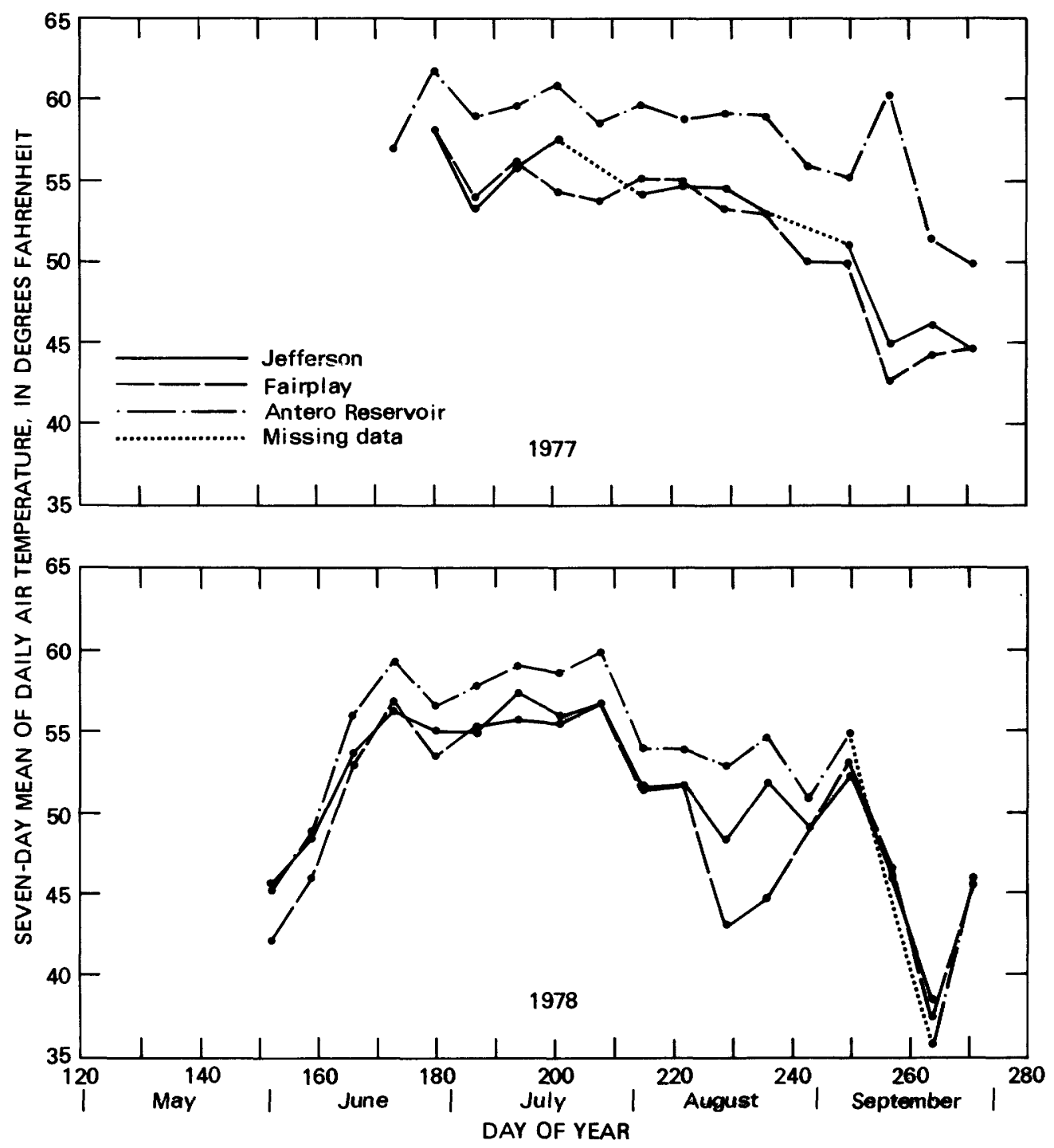

Figure 9.-- Seven-day mean of daily air temperature, Jefferson, Fairplay, and Antero Reservoir station s, May-September 1977 and 1978. 


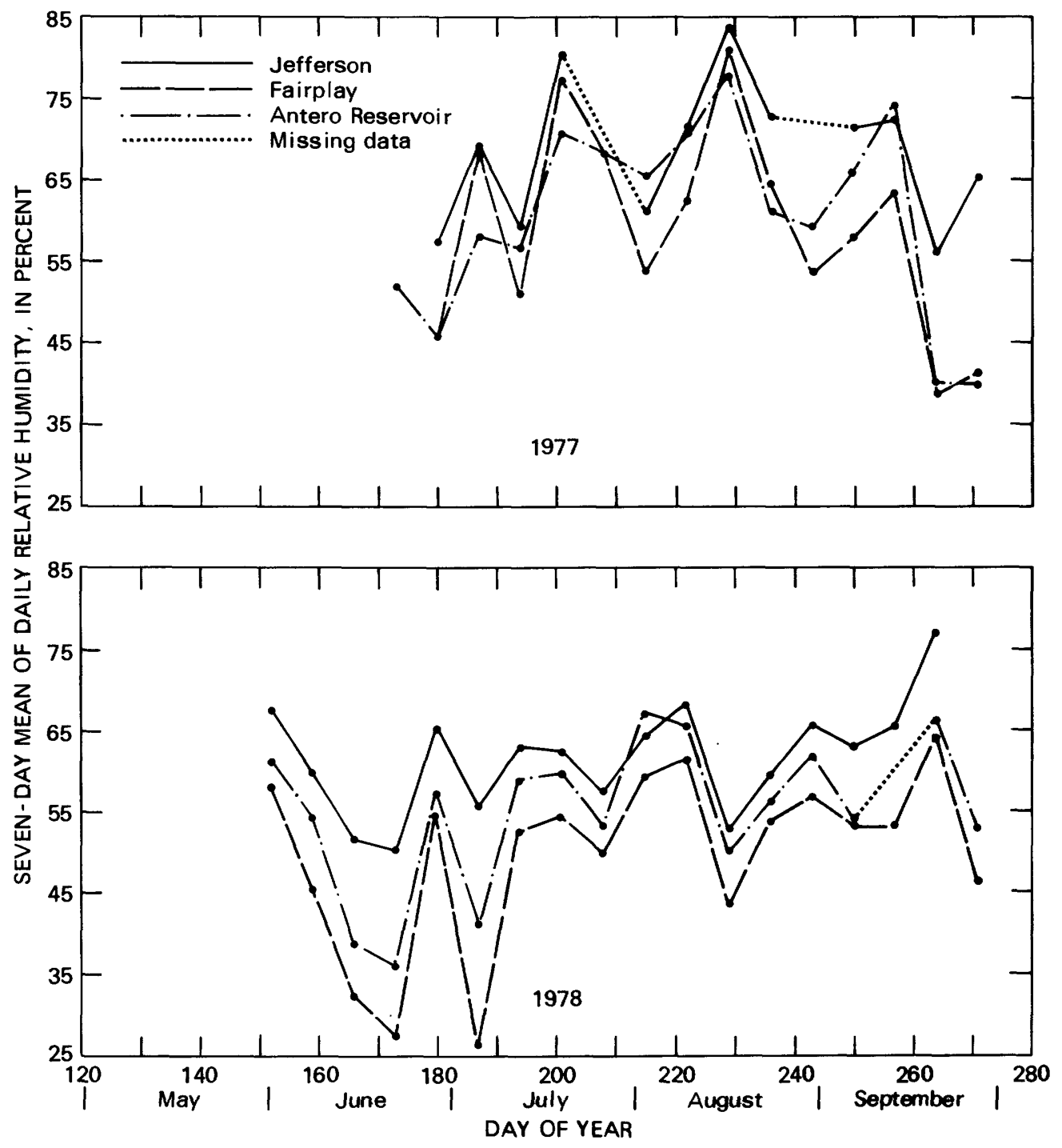

Figure 10.-- Seven-day mean of daily relative humidity, Jefferson, Fairplay, and Antero Reservoir stations, May-September 1977 and 1978. 


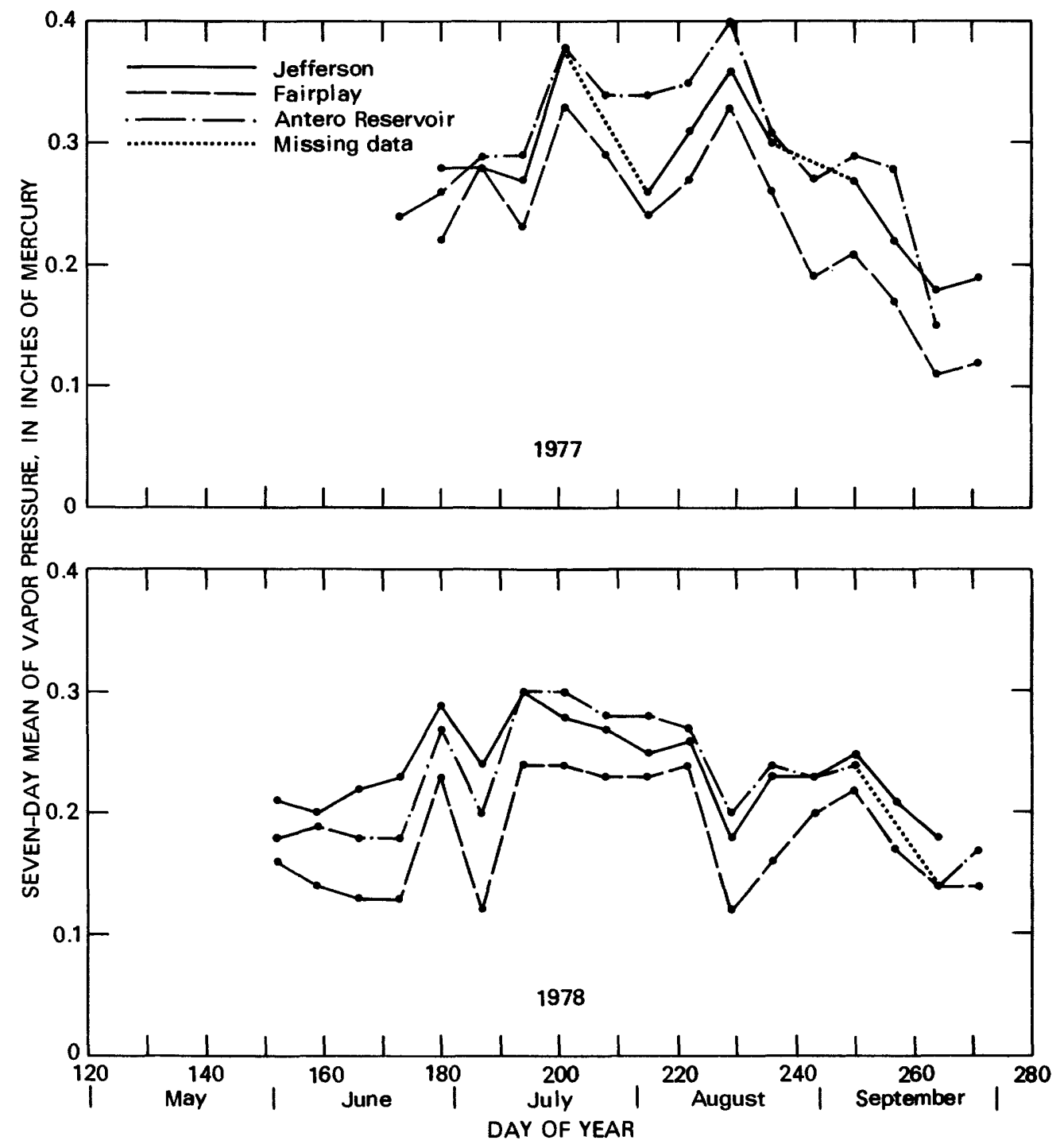

Figure 11.-- Seven-day me an of vapor pressure, Jefferson, Fairplay, and Antero Reservoir stations, May-September 1977 and 1978. 


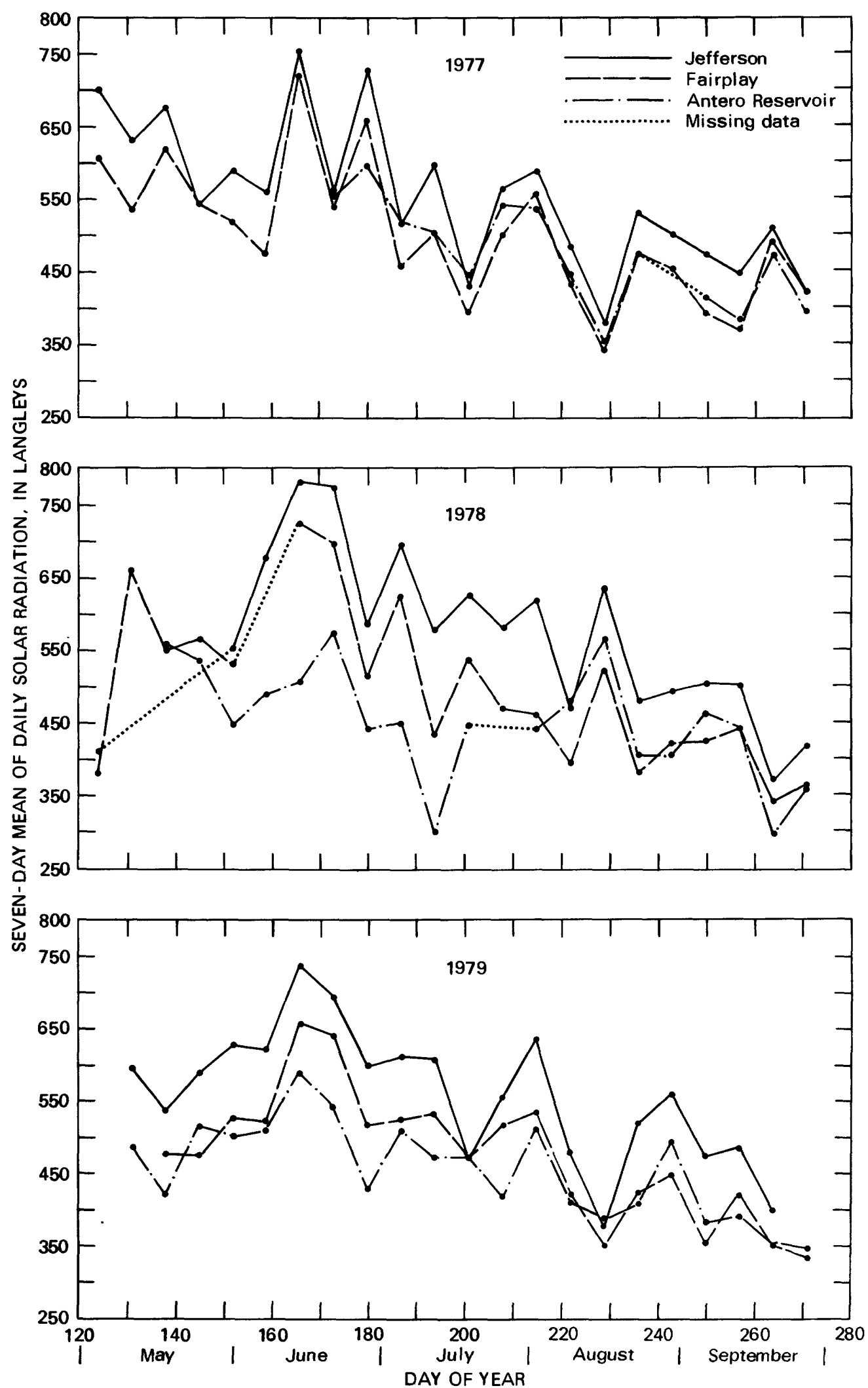

Figure 12.--Seven-day mean of daily solar radiation, Jefferson. Fairplay, and Antero Reservoir stations, May-Sep tember 1977, 1978, and 1979. 
Table 4.--Climatic data, Jefferson station, May-September 1977, 1978, and 1979

TEMPERATURE MAX: Maximum daily air temperature

TEMPERATURE MIN: Minimum daily air temperature

PAN WATER MAX: Maximum daily water temperature of evaporation pan

PAN WATER MIN: Minimum daily water temperature of evaporation pan

PRECIP: Daily precipitation

PAN EVAP: Daily pan evaporation

DAILY AVG TEMP: Daily average air temperature

DAILY AVG REL HUM: Daily average relative humidity

SOLAR RAD: Daily average solar radiation

F: Degrees Fahrenheit

IN: Inches

MI: Mile

LY: Langleys per minute

***: Represents missing data 
Table 4.--Climatic data, Jefferson station,

\begin{tabular}{|c|c|c|c|c|c|c|c|}
\hline $\begin{array}{r}\text { DAY } \\
\text { OF } \\
\text { YEAR }\end{array}$ & MONTH & DAY & YEAR & $\begin{array}{l}\text { TEMP } \\
\text { MAX } \\
\text { (F) }\end{array}$ & $\begin{array}{l}\text { TURE } \\
\text { MIN } \\
\text { (F) }\end{array}$ & $\begin{array}{l}\text { DRY } \\
\text { BULB } \\
\text { (F) }\end{array}$ & $\begin{array}{l}\text { WET } \\
\text { BULB } \\
\text { (F) }\end{array}$ \\
\hline $\begin{array}{l}121 \\
122 \\
123 \\
124 \\
125\end{array}$ & $\begin{array}{l}5 \\
5 \\
5 \\
5 \\
5\end{array}$ & $\begin{array}{l}1 \\
2 \\
3 \\
4 \\
5\end{array}$ & $\begin{array}{l}77 \\
77 \\
17 \\
17 \\
77\end{array}$ & $\begin{array}{l}59 . \\
54 . \\
57 . \\
57 . \\
30 .\end{array}$ & $\begin{array}{l}220^{\circ} \\
200^{\circ} \\
190^{\circ} \\
280^{\circ}\end{array}$ & $\begin{array}{l}40 \\
310^{\circ} \\
380^{\circ} \\
37 \\
35\end{array}$ & $\begin{array}{l}38 . \\
29 . \\
34 . \\
31 . \\
31 .\end{array}$ \\
\hline $\begin{array}{l}126 \\
127 \\
128 \\
129 \\
130\end{array}$ & $\begin{array}{l}5 \\
5 \\
5 \\
5 \\
5\end{array}$ & $\begin{array}{r}6 \\
7 \\
8 \\
9 \\
10\end{array}$ & $\begin{array}{l}77 \\
77 \\
77 \\
77 \\
77\end{array}$ & $\begin{array}{l}58 . \\
60 . \\
63 . \\
64 . \\
65 .\end{array}$ & $\begin{array}{l}200^{\circ} \\
220^{\circ} \\
240^{\circ} \\
230^{\circ}\end{array}$ & $\begin{array}{l}34{ }^{\circ} \\
35{ }^{\circ} \\
40 \\
43 \\
35\end{array}$ & $\begin{array}{l}33 \\
34 \\
38 \\
37 \\
35\end{array}$ \\
\hline $\begin{array}{l}131 \\
132 \\
133 \\
134 \\
135\end{array}$ & $\begin{array}{l}5 \\
5 \\
5 \\
5 \\
5\end{array}$ & $\begin{array}{l}11 \\
12 \\
13 \\
14 \\
15\end{array}$ & $\begin{array}{l}77 \\
77 \\
77 \\
77 \\
77\end{array}$ & $\begin{array}{l}58 . \\
58 . \\
57 . \\
61 . \\
41 .\end{array}$ & $\begin{array}{l}12 . \\
25 . \\
27 . \\
29 . \\
27 .\end{array}$ & $\begin{array}{l}33 \\
31 \\
35 \\
40 \\
35\end{array}$ & $\begin{array}{l}33 \\
310^{\circ} \\
34 \circ^{\circ} \\
38 \\
34\end{array}$ \\
\hline $\begin{array}{l}136 \\
137 \\
138 \\
139 \\
140\end{array}$ & $\begin{array}{l}5 \\
5 \\
5 \\
5 \\
5\end{array}$ & $\begin{array}{l}16 \\
17 \\
18 \\
19 \\
20\end{array}$ & $\begin{array}{l}77 \\
77 \\
77 \\
77 \\
77\end{array}$ & $\begin{array}{l}51 . \\
55{ }^{\circ} \\
56 . \\
53 . \\
48 .\end{array}$ & $\begin{array}{l}210^{\circ} \\
20^{\circ} \\
180^{\circ} \\
14^{\circ} \\
24^{\circ}\end{array}$ & $\begin{array}{l}36 . \\
40 \\
36 . \\
29 \\
29\end{array}$ & $\begin{array}{l}350^{\circ} \\
310^{\circ} \\
330^{\circ} \\
290^{\circ}\end{array}$ \\
\hline $\begin{array}{l}141 \\
142 \\
143 \\
144 \\
145\end{array}$ & $\begin{array}{l}5 \\
5 \\
5 \\
5 \\
5\end{array}$ & $\begin{array}{l}21 \\
22 \\
23 \\
24 \\
25\end{array}$ & $\begin{array}{l}77 \\
77 \\
77 \\
77 \\
77\end{array}$ & $\begin{array}{l}41 . \\
46 . \\
57 \\
63 . \\
57 .\end{array}$ & $\begin{array}{l}25 . \\
18 . \\
20 . \\
24 . \\
25 .\end{array}$ & $\begin{array}{l}33 . \\
24{ }^{\circ} \\
39 \\
350^{\circ} \\
39\end{array}$ & $\begin{array}{l}33 . \\
24 \\
37 \\
33 \\
36\end{array}$ \\
\hline
\end{tabular}


Maý-September 1977, 1978, and 1979--Continued

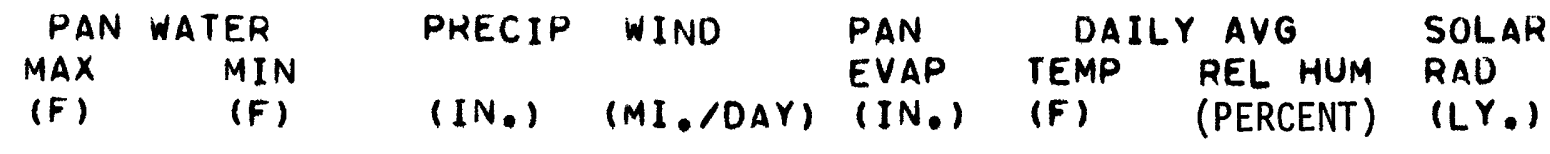

\begin{tabular}{|c|c|c|c|c|c|c|}
\hline $\begin{array}{l}51 . \\
57 . \\
66 . \\
57 \\
57 .\end{array}$ & $\begin{array}{l}37 \\
35{ }^{\circ} \\
32 \\
35 \\
33\end{array}$ & $\begin{array}{ll}0.00 & 0 \\
0.0 & 0 \\
0.0 & 0 \\
0.0 & 0 \\
0.0 & 0\end{array}$ & $\begin{array}{r}115^{\circ} \\
65^{\circ} \\
117^{\circ} \\
144^{\circ} \\
134^{\circ}\end{array}$ & $\begin{array}{r}.21 \\
.23 \\
.21 \\
.26 \\
.28\end{array}$ & 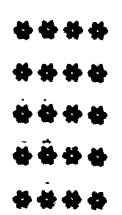 & 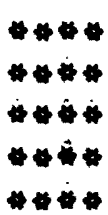 \\
\hline
\end{tabular}

\begin{tabular}{|c|c|c|c|c|c|c|c|}
\hline $\begin{array}{l}61 . \\
66{ }^{\circ} \\
66 . \\
70 . \\
67 .\end{array}$ & $\begin{array}{l}32 . \\
34{ }^{\circ} \\
37{ }^{\circ} \\
37 . \\
38\end{array}$ & $\begin{array}{l}0.00 \\
0.00 \\
0.00 \\
0.00 \\
0.00\end{array}$ & $\begin{array}{r}81 . \\
119 \\
82 \\
95 \\
158\end{array}$ & $\begin{array}{l}.24 \\
.33 \\
.28 \\
.26 \\
.29\end{array}$ & 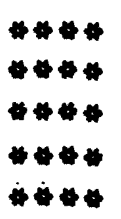 & 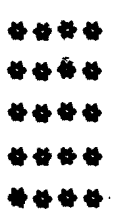 & $\begin{array}{l}752^{\circ} \\
785^{\circ} \\
768^{\circ} \\
674^{\circ} \\
697^{\circ}\end{array}$ \\
\hline
\end{tabular}

\begin{tabular}{|c|c|c|c|c|c|c|c|}
\hline $\begin{array}{l}61 . \\
60 . \\
65 . \\
65 . \\
50 .\end{array}$ & $\begin{array}{l}32 . \\
32 . \\
36 \\
37 \\
32\end{array}$ & $\begin{array}{r}0.00 \\
0.00 \\
0.00 \\
.01 \\
.22\end{array}$ & $\begin{array}{r}105 \\
75^{\circ} \\
74 \\
118 \\
97\end{array}$ & $\begin{array}{r}.29 \\
.20 \\
.21 \\
.07 \\
.18\end{array}$ & 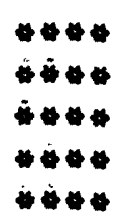 & 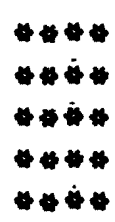 & $\begin{array}{l}729^{\circ} \\
599^{\circ} \\
560^{\circ} \\
386^{\circ} \\
742\end{array}$ \\
\hline
\end{tabular}

\begin{tabular}{|c|c|c|c|c|c|c|}
\hline $\begin{array}{l}63 . \\
60 . \\
60 . \\
58 . \\
62 .\end{array}$ & $\begin{array}{l}33 . \\
35 \circ \\
35 \circ \\
33 . \\
33\end{array}$ & $\begin{array}{l}0.00 \\
0.00 \\
0.00 \\
0.00 \\
0.00\end{array}$ & $\begin{array}{r}141^{\circ} \\
156 . \\
187^{\circ} \\
81 . \\
107^{\circ}\end{array}$ & $\begin{array}{r}.31 \\
.30 \\
.35 \\
.17 \\
.13\end{array}$ & 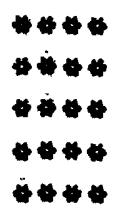 & 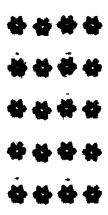 \\
\hline
\end{tabular}

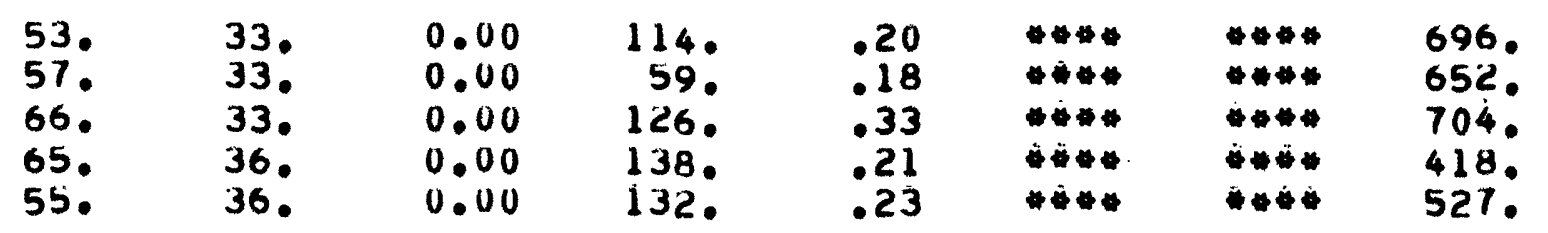


Table 4.--Climatic data, Jefferson station,

\begin{tabular}{|c|c|c|c|c|c|c|c|}
\hline $\begin{array}{r}\text { DAY } \\
\text { OF } \\
\text { YEAR }\end{array}$ & MONTH & DAY & YEAR & $\begin{array}{l}\text { IEMP } \\
\text { MAX } \\
\text { (F) }\end{array}$ & $\begin{array}{l}\text { TURE } \\
\text { MIN } \\
\text { (F) }\end{array}$ & $\begin{array}{l}\text { DRY } \\
\text { BULB } \\
(F)\end{array}$ & $\begin{array}{l}\text { WET } \\
\text { BULB } \\
\text { (F) }\end{array}$ \\
\hline $\begin{array}{l}146 \\
147 \\
148 \\
149 \\
150\end{array}$ & $\begin{array}{l}5 \\
5 \\
5 \\
5 \\
5\end{array}$ & $\begin{array}{l}26 \\
27 \\
28 \\
29 \\
30\end{array}$ & $\begin{array}{l}77 \\
77 \\
77 \\
77 \\
77\end{array}$ & $\begin{array}{l}53 . \\
54 . \\
53 . \\
48 . \\
59 .\end{array}$ & $\begin{array}{l}19 . \\
17{ }^{\circ} \\
20 \\
24{ }^{\circ} \\
27{ }^{\circ}\end{array}$ & $\begin{array}{l}35 \\
38 \\
35 \\
35 \\
38\end{array}$ & $\begin{array}{l}34 \\
36 \\
35 \\
35 \\
38\end{array}$ \\
\hline $\begin{array}{l}151 \\
152 \\
153 \\
154 \\
155\end{array}$ & $\begin{array}{l}5 \\
6 \\
6 \\
6 \\
6\end{array}$ & $\begin{array}{r}31 \\
1 \\
2 \\
3 \\
4\end{array}$ & $\begin{array}{l}77 \\
77 \\
77 \\
77 \\
77\end{array}$ & $\begin{array}{l}64 . \\
69 . \\
69 . \\
10 . \\
67 .\end{array}$ & $\begin{array}{l}31 . \\
27 . \\
29 . \\
28 . \\
29 .\end{array}$ & $\begin{array}{l}50^{\circ} \\
35{ }^{\circ} \\
49^{\circ} \\
49^{\circ} \\
50^{\circ}\end{array}$ & $\begin{array}{l}46 \\
35^{\circ} \\
43{ }^{\circ} \\
43 \\
45^{\circ}\end{array}$ \\
\hline $\begin{array}{l}156 \\
157 \\
158 \\
159 \\
160\end{array}$ & $\begin{array}{l}6 \\
6 \\
6 \\
6 \\
6\end{array}$ & $\begin{array}{l}5 \\
6 \\
7 \\
8 \\
9\end{array}$ & $\begin{array}{l}77 \\
77 \\
77 \\
77 \\
77\end{array}$ & $\begin{array}{l}71 . \\
72 . \\
62 . \\
68 . \\
65 .\end{array}$ & $\begin{array}{l}33 . \\
39 . \\
33 . \\
33 . \\
33 .\end{array}$ & $\begin{array}{l}58^{\circ} \\
45^{\circ} \\
44^{\circ} \\
40^{\circ} \\
47^{\circ}\end{array}$ & $\begin{array}{l}49 . \\
44 . \\
42 . \\
39 \\
44 .\end{array}$ \\
\hline $\begin{array}{l}161 \\
162 \\
163 \\
164 \\
165\end{array}$ & $\begin{array}{l}6 \\
6 \\
6 \\
6 \\
6\end{array}$ & $\begin{array}{l}10 \\
11 \\
12 \\
13 \\
14\end{array}$ & $\begin{array}{l}77 \\
77 \\
77 \\
77 \\
77\end{array}$ & $\begin{array}{l}62 . \\
65 . \\
63 . \\
67 . \\
69 .\end{array}$ & $\begin{array}{l}28 . \\
32 . \\
29 \\
35 . \\
35 .\end{array}$ & $\begin{array}{l}45 . \\
54{ }^{\circ} \\
46 . \\
54{ }^{\circ} \\
54 .\end{array}$ & $\begin{array}{l}410^{\circ} \\
45^{\circ} \\
43 \\
48 \\
49\end{array}$ \\
\hline $\begin{array}{l}166 \\
167 \\
168 \\
169 \\
170\end{array}$ & $\begin{array}{l}6 \\
6 \\
6 \\
6 \\
6\end{array}$ & $\begin{array}{l}15 \\
16 \\
17 \\
18 \\
19\end{array}$ & $\begin{array}{l}77 \\
77 \\
77 \\
77 \\
77\end{array}$ & $\begin{array}{l}10 \\
12 . \\
73 . \\
14 \\
12 .\end{array}$ & $\begin{array}{l}29 . \\
30 . \\
32 . \\
39 . \\
31 .\end{array}$ & $\begin{array}{l}53{ }^{\circ} \\
48{ }^{\circ} \\
51{ }^{\circ} \\
53 . \\
44^{\circ}\end{array}$ & $\begin{array}{l}42 \\
40 \\
41 . \\
50 \\
42\end{array}$ \\
\hline
\end{tabular}


May-September 1977, 1978, and 1979--Continued

\begin{tabular}{|c|c|c|c|c|c|c|c|}
\hline $\begin{array}{l}\text { PAN } \\
\text { MAX } \\
(F)\end{array}$ & $\begin{array}{l}\text { ATER } \\
\text { MIN } \\
(F)\end{array}$ & PRECIP & WIND & $\begin{array}{l}\text { PAN } \\
\text { EVAP } \\
\left(I N_{\bullet}\right)\end{array}$ & $\begin{array}{l}\text { DAILY } \\
\text { TEMP } \\
\text { (F) }\end{array}$ & $\begin{array}{l}\text { YVG } \\
\text { REL HUM } \\
\text { (PERCENT) }\end{array}$ & \\
\hline
\end{tabular}

\begin{tabular}{|c|c|c|c|c|c|c|}
\hline $\begin{array}{l}58 . \\
55 . \\
60 . \\
54 . \\
62 .\end{array}$ & $\begin{array}{l}35 . \\
34 \\
35 \\
35 \\
38\end{array}$ & $\begin{array}{l}0.00 \\
0.00 \\
0.00 \\
0.00 \\
0.00\end{array}$ & $\begin{array}{l}72 . \\
86 . \\
72 . \\
63 . \\
92 .\end{array}$ & $\begin{array}{l}.09 \\
.22 \\
.11 \\
.17 \\
.31\end{array}$ & 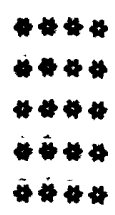 & 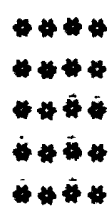 \\
\hline
\end{tabular}

\begin{tabular}{|c|c|c|c|c|c|c|}
\hline $\begin{array}{l}72 . \\
78 \\
70 \\
68 \\
68 .\end{array}$ & $\begin{array}{l}39 . \\
39 . \\
37 . \\
38 . \\
39 .\end{array}$ & $\begin{array}{l}0.00 \\
0.00 \\
0.00 \\
0.00 \\
0.00\end{array}$ & $\begin{array}{l}73 . \\
49 . \\
73 . \\
66 . \\
16 .\end{array}$ & $\begin{array}{l}.33 \\
.23 \\
.24 \\
.20 \\
.25\end{array}$ & 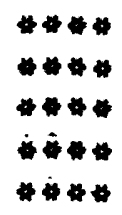 & 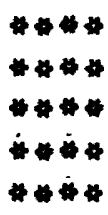 \\
\hline
\end{tabular}

\begin{tabular}{|c|c|c|c|c|c|c|}
\hline $\begin{array}{l}67 . \\
74 \\
68 \\
75 \\
63\end{array}$ & $\begin{array}{l}40 \\
42 \\
42 \\
42 \\
40\end{array}$ & $\begin{array}{r}0.00 \\
.06 \\
0.00 \\
0.00 \\
.40\end{array}$ & $\begin{array}{r}102 \\
55 \\
74 \\
59 \\
68\end{array}$ & $\begin{array}{l}.30 \\
.13 \\
.23 \\
.11 \\
.18\end{array}$ & 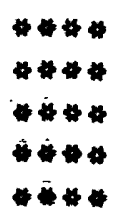 & 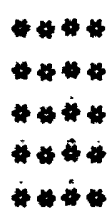 \\
\hline
\end{tabular}

\begin{tabular}{|c|c|c|c|c|c|c|}
\hline $\begin{array}{l}68 . \\
74 . \\
65 . \\
75 . \\
77 .\end{array}$ & $\begin{array}{l}39 . \\
40 . \\
39 . \\
39 . \\
45 .\end{array}$ & $\begin{array}{r}.05 \\
0.00 \\
.05 \\
0.00 \\
.02\end{array}$ & $\begin{array}{l}87 . \\
80 . \\
65 . \\
57 . \\
64 .\end{array}$ & $\begin{array}{r}.34 \\
.20 \\
.30 \\
.26 \\
.34\end{array}$ & 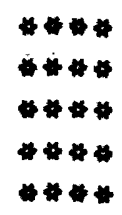 & 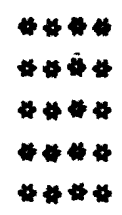 \\
\hline
\end{tabular}

\begin{tabular}{|c|c|c|c|c|c|c|}
\hline $\begin{array}{l}78 . \\
75 . \\
77: \\
76 . \\
74 .\end{array}$ & $\begin{array}{l}39 . \\
39 . \\
39 . \\
39 . \\
45 .\end{array}$ & $\begin{array}{l}0.00 \\
0.00 \\
0.00 \\
0.00 \\
0.00\end{array}$ & $\begin{array}{l}80 . \\
82 . \\
58 . \\
85 . \\
60 .\end{array}$ & $\begin{array}{r}.36 \\
.33 \\
.28 \\
.35 \\
.23\end{array}$ & 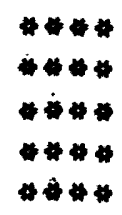 & 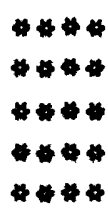 \\
\hline
\end{tabular}


Table 4.--Climatic data, Jefferson station,

\begin{tabular}{|c|c|c|c|c|c|c|c|}
\hline $\begin{array}{r}\text { DAY } \\
\text { OF } \\
\text { YEAR }\end{array}$ & MONTH & DAY & YEAR & $\begin{array}{l}\text { TEMF } \\
\text { MAX } \\
\text { (F) }\end{array}$ & $\begin{array}{l}\text { TUHE } \\
\text { MIN } \\
\text { (F) }\end{array}$ & $\begin{array}{l}\text { DRY } \\
\text { BULB } \\
\text { (F) }\end{array}$ & $\begin{array}{l}\text { WET } \\
\text { BULB } \\
\text { (F) }\end{array}$ \\
\hline $\begin{array}{l}171 \\
172 \\
173 \\
174 \\
175\end{array}$ & $\begin{array}{l}6 \\
6 \\
6 \\
6 \\
6\end{array}$ & $\begin{array}{l}20 \\
21 \\
22 \\
23 \\
24\end{array}$ & $\begin{array}{l}17 \\
17 \\
17 \\
77 \\
77\end{array}$ & $\begin{array}{l}11 \\
68 \\
66 \\
71 \\
63\end{array}$ & $\begin{array}{l}34 \\
27 \\
29 \\
35^{\circ} \\
34\end{array}$ & $\begin{array}{l}53 . \\
49 . \\
47 . \\
47 . \\
50 .\end{array}$ & $\begin{array}{l}50 \\
40 \\
42 . \\
43 . \\
46 .\end{array}$ \\
\hline $\begin{array}{l}176 \\
177 \\
178 \\
179 \\
180\end{array}$ & $\begin{array}{l}6 \\
6 \\
6 \\
6 \\
6\end{array}$ & $\begin{array}{l}25 \\
26 \\
27 \\
28 \\
29\end{array}$ & $\begin{array}{l}77 \\
17 \\
77 \\
77 \\
77\end{array}$ & $\begin{array}{l}63 . \\
71 \\
72 \\
73 . \\
14\end{array}$ & $\begin{array}{l}31 \\
37 \\
33 \\
42 \\
36\end{array}$ & $\begin{array}{l}45 . \\
59 \\
55 . \\
52 \\
48 .\end{array}$ & $\begin{array}{l}43 \\
49^{\circ} \\
46 \\
45^{\circ} \\
46^{\circ}\end{array}$ \\
\hline $\begin{array}{l}181 \\
182 \\
183 \\
184 \\
185\end{array}$ & $\begin{array}{l}6 \\
7 \\
7 \\
7 \\
7\end{array}$ & $\begin{array}{r}30 \\
1 \\
2 \\
3 \\
4\end{array}$ & $\begin{array}{l}77 \\
77 \\
77 \\
77 \\
77\end{array}$ & $\begin{array}{l}13 \\
72 \\
72 \\
75 \\
13\end{array}$ & $\begin{array}{l}35 \\
30 \\
40 \\
36 \\
39\end{array}$ & $\begin{array}{l}45 . \\
46 . \\
57 \\
59 \\
55 .\end{array}$ & $\begin{array}{l}42 \\
42 \\
46 \\
50 \\
52\end{array}$ \\
\hline $\begin{array}{l}186 \\
187 \\
188 \\
189 \\
190\end{array}$ & $\begin{array}{l}7 \\
7 \\
7 \\
7 \\
7\end{array}$ & $\begin{array}{l}5 \\
6 \\
7 \\
8 \\
9\end{array}$ & $\begin{array}{l}17 \\
77 \\
77 \\
77 \\
17\end{array}$ & $\begin{array}{l}73 . \\
69 . \\
66 . \\
69 . \\
67 .\end{array}$ & $\begin{array}{l}37 . \\
30 \\
33 . \\
29 \\
31 .\end{array}$ & $\begin{array}{l}55^{\circ} \\
48^{\circ} \\
49^{\circ} \\
45^{\circ} \\
52^{\circ}\end{array}$ & $\begin{array}{l}48 \\
45 \\
44 \\
42 \\
46\end{array}$ \\
\hline $\begin{array}{l}191 \\
192 \\
193 \\
194 \\
195\end{array}$ & $\begin{array}{l}7 \\
7 \\
7 \\
7 \\
7\end{array}$ & $\begin{array}{l}10 \\
11 \\
12 \\
13 \\
14\end{array}$ & $\begin{array}{l}77 \\
77 \\
77 \\
77 \\
77\end{array}$ & $\begin{array}{l}66 . \\
71 . \\
71 . \\
15 . \\
69 .\end{array}$ & $\begin{array}{l}30 \\
30 \\
33 \\
36 \\
32\end{array}$ & $\begin{array}{l}52 . \\
45 . \\
54 . \\
53 \\
54 .\end{array}$ & $\begin{array}{l}45 \\
38 \\
44 \\
46 \\
47\end{array}$ \\
\hline
\end{tabular}


May-September 1977, 1978, and 1979--Continued

\begin{tabular}{|c|c|c|c|c|c|}
\hline $\begin{array}{l}\text { PAN } \\
\text { MAX } \\
\text { (F) }\end{array}$ & $\begin{array}{l}\text { WATER } \\
\text { MIN } \\
\text { (F) }\end{array}$ & & (MI./DAY) & $\begin{array}{l}\text { PAN } \\
\text { EVAP } \\
\text { (IN.) }\end{array}$ & $\begin{array}{l}\text { DAILY AVG } \\
\text { TEMP REL HUM } \\
\text { (F) } \\
\text { (PERCENT) }\end{array}$ \\
\hline
\end{tabular}

\begin{tabular}{|c|c|c|c|c|c|c|}
\hline $\begin{array}{l}72 . \\
67 . \\
68 . \\
81 . \\
59\end{array}$ & $\begin{array}{l}45 . \\
39 . \\
39 . \\
45 . \\
42 .\end{array}$ & $\begin{array}{l}0.00 \\
0.00 \\
0.00 \\
0.00 \\
0.00\end{array}$ & $\begin{array}{l}96 . \\
62 . \\
49 . \\
42 . \\
54 .\end{array}$ & $\begin{array}{l}.27 \\
.25 \\
.16 \\
.22 \\
.15\end{array}$ & $\begin{array}{l}4 \$ 4 \\
50 . \\
53 . \\
50 . \\
51 .\end{array}$ & $\begin{array}{l}* 4 \% \\
* 4 \% \\
54 . \\
74 . \\
70 .\end{array}$ \\
\hline
\end{tabular}

$\begin{array}{llllllll}62 . & 41 . & 0.00 & 54 . & .22 & 52 . & 64 . & 530 . \\ 71 . & 42 . & 0.00 & 114 . & .36 & 58 . & 56 . & 780 . \\ 77 . & 43 . & 0.00 & 115 . & .41 & 51 . & 58 . & 815 . \\ 78 . & 44 . & 0.00 & 47 . & .25 & 59 . & 61 . & 671 . \\ 81 . & 46 . & 0.00 & 88 . & .25 & 59 . & 56 . & 684 . \\ & & & & & & & \\ 76 . & 40 . & 0.00 & 71 . & .31 & 56 . & 57 . & 795 . \\ 75 . & 41 . & 0.00 & 58 . & .28 & 57 . & 63 . & 608 . \\ 72 . & 43 . & 0.00 & 95 . & .36 & 61 . & 51 . & 723 . \\ 75 . & 45 . & 0.00 & 50 . & .23 & 59 . & 66 . & 511 . \\ 75 . & 47 . & .15 & 66 . & .27 & 58 . & 69 . & 562 . \\ & & & & & & & \\ 73 . & 47 . & 0.00 & 69 . & .20 & 54 . & 72 . & 531 . \\ 75 . & 41 . & .07 & 58 . & .17 & 49 . & 74 . & 428 . \\ 66 . & 40 . & .02 & 44 . & .22 & 53 . & 60 . & 536 . \\ 73 . & 40 . & 0.00 & 46 . & .12 & 50 . & 70 . & 518 . \\ 71 . & 40 . & 0.00 & 58 . & .44 & 51 . & 75 . & 537 . \\ & & & & & & & \\ 65 . & 40 . & .16 & 87 . & .38 & 55 . & 55 . & 815 . \\ 73 . & 38 . & 0.00 & 75 . & .29 & 54 . & 40 . & 583 . \\ 67 . & 39 . & 0.00 & 51 . & .29 & 56 . & 45 . & 708 . \\ 80 . & 40 . & 0.00 & 61 . & .14 & 54 . & 74 . & 412 . \\ 76 . & 42 . & .02 & 45 . & .20 & 56 . & 71 . & 541 .\end{array}$


Table 4.--Climatic data, Jefferson station,

\begin{tabular}{|c|c|c|c|c|c|c|c|}
\hline $\begin{array}{r}\text { DAY } \\
\text { OF } \\
\text { YEAR }\end{array}$ & MONTH & DAY & YEAR & $\begin{array}{l}\text { TEMF } \\
\text { MAX } \\
\text { (F) }\end{array}$ & $\begin{array}{l}\text { URE } \\
\text { MIN } \\
\text { (F) }\end{array}$ & $\begin{array}{l}\text { DRY } \\
\text { BULB } \\
\text { (F) }\end{array}$ & $\begin{array}{l}\text { WET } \\
\text { BULL } \\
\text { (F) }\end{array}$ \\
\hline $\begin{array}{l}196 \\
197 \\
198 \\
199 \\
200\end{array}$ & $\begin{array}{l}7 \\
7 \\
7 \\
7 \\
7\end{array}$ & $\begin{array}{l}15 \\
16 \\
17 \\
18 \\
19\end{array}$ & $\begin{array}{l}77 \\
77 \\
77 \\
77 \\
77\end{array}$ & $\begin{array}{l}10 . \\
72 . \\
16 . \\
77 . \\
16 .\end{array}$ & $\begin{array}{l}31 . \\
31 . \\
36 . \\
390^{\circ} \\
43 .\end{array}$ & $\begin{array}{l}40 . \\
50 \\
58 \\
52 . \\
59 .\end{array}$ & $\begin{array}{l}38 \\
46 . \\
49 . \\
47 \\
50\end{array}$ \\
\hline $\begin{array}{l}201 \\
202 \\
203 \\
204 \\
205\end{array}$ & $\begin{array}{l}7 \\
7 \\
7 \\
7 \\
7\end{array}$ & $\begin{array}{l}20 \\
21 \\
22 \\
23 \\
24\end{array}$ & $\begin{array}{l}77 \\
77 \\
17 \\
17 \\
77\end{array}$ & $\begin{array}{l}13 . \\
68 . \\
64 . \\
67 . \\
10 .\end{array}$ & $\begin{array}{l}31 . \\
450^{\circ} \\
450^{\circ} \\
42 . \\
38 .\end{array}$ & $\begin{array}{l}53 \\
50 . \\
49 . \\
55 \\
55 .\end{array}$ & $\begin{array}{l}50 \\
49 . \\
48 . \\
51 . \\
51 .\end{array}$ \\
\hline $\begin{array}{l}206 \\
207 \\
208 \\
209 \\
210\end{array}$ & $\begin{array}{l}7 \\
7 \\
7 \\
7 \\
7\end{array}$ & $\begin{array}{l}25 \\
26 \\
27 \\
28 \\
29\end{array}$ & $\begin{array}{l}77 \\
77 \\
77 \\
77 \\
77\end{array}$ & $\begin{array}{l}69 . \\
63 . \\
72 . \\
70 . \\
68 .\end{array}$ & $\begin{array}{l}45 . \\
41 . \\
38 . \\
33 . \\
35 .\end{array}$ & $\begin{array}{l}48 \\
52 \\
55 \\
45 \\
49\end{array}$ & $\begin{array}{l}47 \\
50 \\
49 \\
43 \\
46 .\end{array}$ \\
\hline $\begin{array}{l}211 \\
212 \\
213 \\
214 \\
215\end{array}$ & $\begin{array}{l}7 \\
7 \\
8 \\
8 \\
8\end{array}$ & $\begin{array}{r}30 \\
31 \\
1 \\
2 \\
3\end{array}$ & $\begin{array}{l}77 \\
77 \\
77 \\
77 \\
77\end{array}$ & $\begin{array}{l}73 . \\
13 . \\
10 . \\
11 . \\
72 .\end{array}$ & $\begin{array}{l}36 . \\
35 . \\
32 . \\
30 \\
33 .\end{array}$ & $\begin{array}{l}51 . \\
51 . \\
50 . \\
52 . \\
53 .\end{array}$ & $\begin{array}{l}45 \\
43 \\
47 \\
42 \\
45 \\
\end{array}$ \\
\hline $\begin{array}{l}216 \\
217 \\
218 \\
219 \\
220\end{array}$ & $\begin{array}{l}8 \\
8 \\
8 \\
8 \\
8\end{array}$ & $\begin{array}{l}4 \\
5 \\
6 \\
7 \\
8\end{array}$ & $\begin{array}{l}77 \\
77 \\
77 \\
77 \\
17\end{array}$ & $\begin{array}{l}15 . \\
68 . \\
65 . \\
73 . \\
75 .\end{array}$ & $\begin{array}{l}37 . \\
41 . \\
40 . \\
360^{\circ} \\
36 .\end{array}$ & $\begin{array}{l}55 \\
48 \\
51 \\
52 \\
49\end{array}$ & $\begin{array}{l}48 \\
47 \\
49 \\
49 \\
45\end{array}$ \\
\hline
\end{tabular}


May-September 1977, 1978, and 1979--Continued

\begin{tabular}{|c|c|c|c|c|c|c|c|}
\hline $\begin{array}{l}\text { PAN } \\
\text { MAX } \\
(F)\end{array}$ & $\begin{array}{l}\text { WATER } \\
\text { MIN } \\
\text { (F) }\end{array}$ & $\begin{array}{l}\text { PRECIP } \\
(I N \cdot)\end{array}$ & $\begin{array}{l}\text { WINO } \\
\text { (MI./DAY) }\end{array}$ & $\begin{array}{l}\text { PAN } \\
\text { EVAP } \\
(I N .)\end{array}$ & $\begin{array}{l}\text { DAI } \\
\text { TEMP } \\
\text { (F) }\end{array}$ & $\begin{array}{l}\text { Y AVG } \\
\text { REL HUM } \\
\text { (PERCENT) }\end{array}$ & $\begin{array}{l}\text { SOLAR } \\
\text { RAD } \\
(L Y .)\end{array}$ \\
\hline $\begin{array}{l}72 . \\
72 . \\
78 . \\
76 . \\
76 .\end{array}$ & $\begin{array}{l}43 . \\
410^{\circ} \\
42^{\circ} \\
45^{\circ} \\
48 .\end{array}$ & $\begin{array}{l}0.00 \\
0.00 \\
0.00 \\
0.00 \\
0.00\end{array}$ & $\begin{array}{l}48 . \\
52 . \\
45 . \\
54 \\
56 .\end{array}$ & $\begin{array}{l}.18 \\
.27 \\
.23 \\
.24 \\
.25\end{array}$ & $\begin{array}{l}55 . \\
60 . \\
60 . \\
60 . \\
59 .\end{array}$ & $\begin{array}{l}72 . \\
57 \\
60 \\
66 \\
77\end{array}$ & $\begin{array}{l}501 . \\
613 . \\
524 . \\
474 . \\
516^{\circ}\end{array}$ \\
\hline $\begin{array}{l}76 \\
61 \\
65 \\
71 \\
78\end{array}$ & $\begin{array}{l}48 . \\
48 . \\
51 . \\
41 . \\
50\end{array}$ & $\begin{array}{l}.34 \\
.24 \\
.66 \\
.07 \\
.11\end{array}$ & $\begin{array}{l}24 \\
17 \\
36 \\
38 \\
35\end{array}$ & $\begin{array}{l}.09 \\
.08 \\
.12 \\
.25 \\
.14\end{array}$ & $\begin{array}{l}55 . \\
55 . \\
56 . \\
58 . \\
56 .\end{array}$ & $\begin{array}{l}98 . \\
97 . \\
90 . \\
75 \\
86 .\end{array}$ & $\begin{array}{l}311 . \\
255 \\
419 . \\
528 \\
507\end{array}$ \\
\hline $\begin{array}{l}75 . \\
69 \\
79 \\
72 \\
72 .\end{array}$ & $\begin{array}{l}51 . \\
50 \\
47 . \\
45 . \\
45 .\end{array}$ & $\begin{array}{r}.02 \\
.10 \\
.13 \\
.02 \\
0.00\end{array}$ & $\begin{array}{l}17 . \\
57 \\
38 . \\
64 . \\
70\end{array}$ & $\begin{array}{l}.07 \\
.34 \\
.19 \\
.25 \\
.29\end{array}$ & $\begin{array}{r}b 2 . \\
* * * \\
* 4 * * \\
52 . \\
b 5 .\end{array}$ & $\begin{array}{r}97 . \\
404 \\
44 * 4 \\
70 . \\
63 .\end{array}$ & $\begin{array}{l}337 \\
603 \\
505 \\
605 \\
734\end{array}$ \\
\hline $\begin{array}{l}78 . \\
76 . \\
76 . \\
78 \\
77\end{array}$ & $\begin{array}{l}45 . \\
39 . \\
42 . \\
39 . \\
39 .\end{array}$ & $\begin{array}{l}0.00 \\
0.00 \\
0.00 \\
0.00 \\
0.00\end{array}$ & $\begin{array}{l}87 . \\
58 . \\
59 . \\
49 . \\
69 .\end{array}$ & $\begin{array}{r}.34 \\
.31 \\
.34 \\
.23 \\
.28\end{array}$ & $\begin{array}{l}57 . \\
57 . \\
45 . \\
54 . \\
57 .\end{array}$ & $\begin{array}{l}53 . \\
51 . \\
61 . \\
52 . \\
39\end{array}$ & $\begin{array}{l}684 . \\
762 . \\
744 . \\
661 . \\
530\end{array}$ \\
\hline $\begin{array}{l}70 . \\
65^{\circ} \\
64{ }^{\circ} \\
77 . \\
74 .\end{array}$ & $\begin{array}{l}41 . \\
45 . \\
48 . \\
47 . \\
45 .\end{array}$ & $\begin{array}{r}0.00 \\
.12 \\
0.00 \\
0.00 \\
0.00\end{array}$ & $\begin{array}{l}33 . \\
69 . \\
55 . \\
63 . \\
41 .\end{array}$ & $\begin{array}{r}.13 \\
.13 \\
.28 \\
.27 \\
.15\end{array}$ & $\begin{array}{l}54 . \\
54 . \\
59 . \\
57 . \\
57 .\end{array}$ & $\begin{array}{l}81 . \\
76 . \\
68 . \\
65 . \\
73 .\end{array}$ & $\begin{array}{l}405 . \\
359 . \\
660 . \\
559 . \\
386 .\end{array}$ \\
\hline
\end{tabular}


Table 4.--Climatic data, Jefferson station,

\begin{tabular}{|c|c|c|c|c|c|c|c|}
\hline $\begin{array}{c}\text { DAY } \\
\text { OF } \\
\text { YEAR }\end{array}$ & MONTH & DAY & YEAR & $\begin{array}{l}\text { IEMF } \\
\text { MAX } \\
\text { (F) }\end{array}$ & $\begin{array}{l}\text { URE } \\
\text { MIN } \\
\text { (F) }\end{array}$ & $\begin{array}{l}\text { DRY } \\
\text { BULB } \\
\text { (F) }\end{array}$ & $\begin{array}{l}\text { WET } \\
\text { BULB } \\
\text { (F) }\end{array}$ \\
\hline $\begin{array}{l}221 \\
222 \\
223 \\
224 \\
225\end{array}$ & $\begin{array}{l}8 \\
8 \\
8 \\
8 \\
8\end{array}$ & $\begin{array}{l}9 \\
10 \\
11 \\
12 \\
13\end{array}$ & $\begin{array}{l}17 \\
77 \\
77 \\
77 \\
77\end{array}$ & $\begin{array}{l}12 . \\
76 . \\
67 \\
10 \\
11 .\end{array}$ & $\begin{array}{l}35 . \\
48 . \\
37 . \\
35 . \\
46 .\end{array}$ & $\begin{array}{l}480^{\circ} \\
50^{\circ} \\
50^{\circ} \\
48{ }^{\circ} \\
49^{\circ}\end{array}$ & $\begin{array}{l}44{ }^{\circ} \\
48^{\circ} \\
49^{\circ} \\
44 \circ^{\circ}\end{array}$ \\
\hline $\begin{array}{l}226 \\
227 \\
228 \\
229 \\
230\end{array}$ & $\begin{array}{l}8 \\
8 \\
8 \\
8 \\
8\end{array}$ & $\begin{array}{l}14 \\
15 \\
16 \\
17 \\
18\end{array}$ & $\begin{array}{l}77 \\
77 \\
77 \\
77 \\
77\end{array}$ & $\begin{array}{l}68 . \\
70 . \\
640^{\circ} \\
65 . \\
63 .\end{array}$ & $\begin{array}{l}42 . \\
41 . \\
48 . \\
41 . \\
47 .\end{array}$ & $\begin{array}{l}46 . \\
51 . \\
51 . \\
51 . \\
58 .\end{array}$ & $\begin{array}{l}44 \\
48 \\
49 \\
48 \\
53\end{array}$ \\
\hline $\begin{array}{l}231 \\
232 \\
233 \\
234 \\
235\end{array}$ & $\begin{array}{l}8 \\
8 \\
8 \\
8 \\
8\end{array}$ & $\begin{array}{l}19 \\
20 \\
21 \\
22 \\
23\end{array}$ & $\begin{array}{l}77 \\
77 \\
77 \\
77 \\
77\end{array}$ & $\begin{array}{l}67 . \\
67 . \\
68 . \\
64 . \\
68 .\end{array}$ & $\begin{array}{l}41 . \\
40 \\
37 \\
350^{\circ} \\
39 .\end{array}$ & $\begin{array}{l}550^{\circ} \\
510^{\circ} \\
470^{\circ} \\
\end{array}$ & $\begin{array}{l}52 \\
48 \\
44{ }^{\circ} \\
46 \\
45^{\circ}\end{array}$ \\
\hline $\begin{array}{l}236 \\
237 \\
238 \\
239 \\
240\end{array}$ & $\begin{array}{l}8 \\
8 \\
8 \\
8 \\
8\end{array}$ & $\begin{array}{l}24 \\
25 \\
26 \\
27 \\
28\end{array}$ & $\begin{array}{l}77 \\
77 \\
77 \\
77 \\
77\end{array}$ & $\begin{array}{l}11 . \\
61 . \\
68 . \\
730 \\
57 .\end{array}$ & $\begin{array}{l}36 . \\
34 . \\
32 . \\
35 . \\
29 .\end{array}$ & $\begin{array}{l}470^{\circ} \\
470^{\circ} \\
390^{\circ} \\
310^{\circ}\end{array}$ & $\begin{array}{l}43 \\
45 \\
36 \\
38 \\
36\end{array}$ \\
\hline $\begin{array}{l}241 \\
242 \\
243 \\
244 \\
245\end{array}$ & $\begin{array}{l}8 \\
8 \\
8 \\
9 \\
9\end{array}$ & $\begin{array}{r}29 \\
30 \\
31 \\
1 \\
2\end{array}$ & $\begin{array}{l}77 \\
77 \\
77 \\
77 \\
77\end{array}$ & $\begin{array}{l}59 . \\
69 . \\
10 . \\
67 . \\
12 .\end{array}$ & $\begin{array}{l}29 . \\
27 . \\
32 . \\
31 . \\
33 .\end{array}$ & $\begin{array}{l}37 \\
38 \\
43 \\
43 \\
49\end{array}$ & $\begin{array}{l}36 \\
34 \\
38 \\
41 \\
43\end{array}$ \\
\hline
\end{tabular}


May-September 1977, 1978, 1979--Continued.

\begin{tabular}{|c|c|c|c|c|c|c|c|}
\hline $\begin{array}{l}\text { PAN } \\
\text { MAX } \\
\text { (F) }\end{array}$ & $\begin{array}{l}\text { WATER } \\
\text { MIN } \\
\text { (F) }\end{array}$ & $\begin{array}{l}\text { PRECIP } \\
\text { (IN.) }\end{array}$ & $\begin{array}{l}\text { WIND } \\
(M I \bullet / D \wedge Y)\end{array}$ & $\begin{array}{l}\text { PAN } \\
\text { EVAP } \\
(I N \cdot)\end{array}$ & $\begin{array}{l}\text { DAI } \\
\text { TEMP } \\
\text { (F) }\end{array}$ & $\begin{array}{l}\text { Y AVG } \\
\text { REL HUM } \\
\text { (PERCENT) }\end{array}$ & $\begin{array}{l}\text { SOLAR } \\
\text { RAD } \\
(L Y .)\end{array}$ \\
\hline $\begin{array}{l}72 . \\
74 . \\
70 . \\
76 \\
72 .\end{array}$ & $\begin{array}{l}46 . \\
44 \\
48 \\
43 \\
42\end{array}$ & $\begin{array}{r}0.00 \\
0.00 \\
.02 \\
0.00 \\
0.00\end{array}$ & $\begin{array}{l}32 . \\
33 . \\
47 \\
60 \\
41 .\end{array}$ & $\begin{array}{l}.07 \\
.24 \\
.24 \\
.26 \\
.14\end{array}$ & $\begin{array}{l}56 . \\
53 \\
53 \\
54 \\
53\end{array}$ & $\begin{array}{l}68 \\
85 \\
70 \\
62 . \\
70\end{array}$ & $\begin{array}{l}481 . \\
377^{\circ} \\
569 . \\
566 . \\
459 .\end{array}$ \\
\hline $\begin{array}{l}72 \\
67 \\
66 \\
72 \\
65\end{array}$ & $\begin{array}{l}42 . \\
44 \circ \\
49 . \\
50 \\
49 .\end{array}$ & $\begin{array}{l}.01 \\
.12 \\
.59 \\
.27 \\
.14\end{array}$ & $\begin{array}{l}41 . \\
37 . \\
34 . \\
18 . \\
40 .\end{array}$ & $\begin{array}{l}.17 \\
.12 \\
.17 \\
.05 \\
.16\end{array}$ & $\begin{array}{l}54 . \\
53 . \\
55 . \\
54 \\
57 .\end{array}$ & $\begin{array}{l}75 . \\
82 . \\
90 . \\
92 . \\
84 .\end{array}$ & $\begin{array}{l}459 . \\
361 . \\
310^{\circ} \\
264 . \\
379 .\end{array}$ \\
\hline $\begin{array}{l}70 \\
71 \\
73 \\
65 \\
72 .\end{array}$ & $\begin{array}{l}51 . \\
51 . \\
45 . \\
45 . \\
45 .\end{array}$ & $\begin{array}{r}.20 \\
.02 \\
0.00 \\
.02 \\
0.00\end{array}$ & $\begin{array}{l}55 . \\
66 \\
35 . \\
81 \\
54\end{array}$ & $\begin{array}{l}.15 \\
.18 \\
.11 \\
.19 \\
.24\end{array}$ & $\begin{array}{l}56 . \\
54 . \\
53 . \\
54 \\
57 .\end{array}$ & $\begin{array}{l}85 . \\
77 . \\
81 . \\
85 \\
64\end{array}$ & $\begin{array}{l}422 . \\
449 . \\
356 . \\
602 . \\
551 .\end{array}$ \\
\hline $\begin{array}{l}72 . \\
70 \\
70 \\
72 . \\
69\end{array}$ & $\begin{array}{l}45 . \\
43 . \\
40 \\
39 \\
40\end{array}$ & $\begin{array}{l}0.00 \\
0.00 \\
0.00 \\
0.00 \\
0.00\end{array}$ & $\begin{array}{r}53 \\
56 \\
108 \\
76 \\
53\end{array}$ & $\begin{array}{l}.17 \\
.19 \\
.19 \\
.34 \\
.06\end{array}$ & $\begin{array}{l}53 . \\
52 . \\
55 \\
49 . \\
46 .\end{array}$ & $\begin{array}{l}75 . \\
75 \\
60 \\
70 \\
70\end{array}$ & $\begin{array}{l}462 . \\
428 \\
647 \\
654 \\
452 .\end{array}$ \\
\hline $\begin{array}{l}61 . \\
75 \\
66 \\
65 \\
74\end{array}$ & $\begin{array}{l}40 . \\
38 . \\
38 . \\
39 . \\
43 .\end{array}$ & $\begin{array}{l}0.00 \\
0.00 \\
0.00 \\
0.00 \\
0.00\end{array}$ & $\begin{array}{l}59 . \\
74 . \\
48 . \\
61 . \\
34 .\end{array}$ & $\begin{array}{l}.34 \\
.25 \\
.15 \\
.26 \\
.16\end{array}$ & $\begin{array}{c}+4 * \\
+4 * \\
51 . \\
54 \\
53 .\end{array}$ & $\begin{array}{r}* 4 * \\
* \$ 4 \\
71 . \\
65 \\
72 .\end{array}$ & $\begin{array}{l}625 . \\
518^{\circ} \\
426 . \\
624 . \\
492 .\end{array}$ \\
\hline
\end{tabular}


Table 4.--Climatic data, Jefferson station,

\begin{tabular}{|c|c|c|c|c|c|c|c|}
\hline $\begin{array}{r}\text { DAY } \\
\text { OF } \\
\text { YEAR }\end{array}$ & MONTH & DAY & YEAR & $\begin{array}{l}\text { TEMF } \\
\text { MAX } \\
\text { (F) }\end{array}$ & $\begin{array}{l}\text { TURE } \\
\text { MIN } \\
\text { (F) }\end{array}$ & $\begin{array}{l}\text { DRY } \\
\text { BULB } \\
\text { (F) }\end{array}$ & $\begin{array}{l}\text { WET } \\
\text { BULB } \\
\text { (F) }\end{array}$ \\
\hline $\begin{array}{l}246 \\
247 \\
248 \\
249 \\
250\end{array}$ & $\begin{array}{l}9 \\
9 \\
9 \\
9 \\
9\end{array}$ & $\begin{array}{l}3 \\
4 \\
5 \\
6 \\
7\end{array}$ & $\begin{array}{l}77 \\
77 \\
77 \\
77 \\
77\end{array}$ & $\begin{array}{l}12 . \\
69 . \\
62 . \\
70 . \\
13 .\end{array}$ & $\begin{array}{l}330^{\circ} \\
36{ }^{\circ} \\
310^{\circ} \\
35 \circ\end{array}$ & $\begin{array}{l}41 \\
42 \\
42 \\
42 \\
45\end{array}$ & $\begin{array}{l}39 . \\
40 . \\
400^{\circ} \\
390^{\circ} \\
40 .\end{array}$ \\
\hline $\begin{array}{l}251 \\
252 \\
253 \\
254 \\
255\end{array}$ & $\begin{array}{l}9 \\
9 \\
9 \\
9 \\
9\end{array}$ & $\begin{array}{r}8 \\
9 \\
10 \\
11 \\
12\end{array}$ & $\begin{array}{l}77 \\
77 \\
17 \\
77 \\
77\end{array}$ & $\begin{array}{l}13 . \\
11 . \\
67 . \\
68 . \\
55 .\end{array}$ & $\begin{array}{l}310^{\circ} \\
280^{\circ} \\
370^{\circ} \\
41 .\end{array}$ & $\begin{array}{l}43 . \\
30 \\
46 . \\
46 . \\
44 .\end{array}$ & $\begin{array}{l}39 . \\
30 \\
40 . \\
42 . \\
42 .\end{array}$ \\
\hline $\begin{array}{l}256 \\
257 \\
258 \\
259 \\
260\end{array}$ & $\begin{array}{l}9 \\
9 \\
9 \\
9 \\
9\end{array}$ & $\begin{array}{l}13 \\
14 \\
15 \\
16 \\
17\end{array}$ & $\begin{array}{l}17 \\
77 \\
77 \\
77 \\
77\end{array}$ & $\begin{array}{l}39 . \\
31 . \\
65 . \\
60 . \\
61 .\end{array}$ & $\begin{array}{l}28 . \\
20 . \\
30 \circ \\
26 . \\
21 .\end{array}$ & $\begin{array}{l}42 . \\
320^{\circ} \\
390^{\circ} \\
350^{\circ} \\
34 .\end{array}$ & $\begin{array}{l}39 . \\
32 . \\
36 \\
32 \\
31\end{array}$ \\
\hline $\begin{array}{l}261 \\
262 \\
263 \\
264 \\
265\end{array}$ & $\begin{array}{l}9 \\
9 \\
9 \\
9 \\
9\end{array}$ & $\begin{array}{l}18 \\
19 \\
20 \\
21 \\
22\end{array}$ & $\begin{array}{l}77 \\
77 \\
77 \\
17 \\
77\end{array}$ & $\begin{array}{l}61 . \\
64 . \\
68 . \\
67 . \\
62 .\end{array}$ & $\begin{array}{l}22 . \\
23 . \\
27 . \\
26 . \\
21 .\end{array}$ & $\begin{array}{l}36 \\
35 \\
44 \\
40 \\
33\end{array}$ & $\begin{array}{l}32 \\
32 \\
40 \\
36 \\
32\end{array}$ \\
\hline $\begin{array}{l}266 \\
267 \\
268 \\
267 \\
270\end{array}$ & $\begin{array}{l}9 \\
9 \\
9 \\
9 \\
9\end{array}$ & $\begin{array}{l}23 \\
24 \\
25 \\
24 \\
27\end{array}$ & $\begin{array}{l}77 \\
77 \\
77 \\
77 \\
77\end{array}$ & $\begin{array}{l}64 . \\
45 . \\
65 . \\
11 . \\
65 .\end{array}$ & $\begin{array}{l}220^{\circ} \\
260^{\circ} \\
230^{\circ} \\
260^{\circ} \\
24 .\end{array}$ & $\begin{array}{l}28 \\
42 \\
38 \\
41 \\
39\end{array}$ & $\begin{array}{l}28 . \\
33{ }^{\circ} \\
320^{\circ} \\
34{ }^{\circ} \\
34\end{array}$ \\
\hline
\end{tabular}


May-September 1977, 1978, and 1979--Continued

$\begin{array}{ccllllll}\text { PAN WATER } & \text { PRECIP } & \text { WIND } & \text { PAN } & \text { DAILY AVG } & \text { SOLAR } \\ \text { MAX } & \text { MIN } & & & \text { EVAP } & \text { TEMP } & \text { REL HUM RAD } \\ \text { (F) } & \text { (F) } & \text { (IN.) } & \text { (MI./DAY) } & \text { (IN.) } & \text { (F) } & \text { (PERCENT) } & \text { (LY.) }\end{array}$

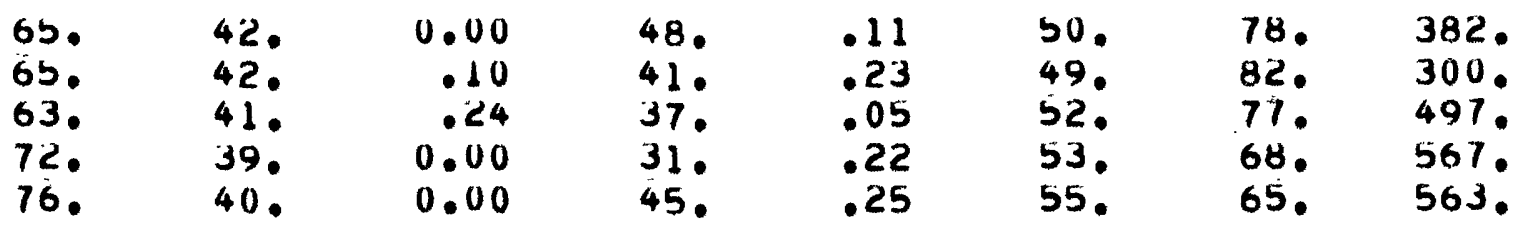

\begin{tabular}{|c|c|c|c|c|c|c|c|}
\hline $\begin{array}{l}75 . \\
63 . \\
61 . \\
61 . \\
55 \circ\end{array}$ & $\begin{array}{l}41{ }^{\circ} \\
38 \circ^{\circ} \\
37 \circ^{\circ} \\
380^{\circ} \\
43 .\end{array}$ & $\begin{array}{l}0.00 \\
0.00 \\
0.00 \\
0.00 \\
.14\end{array}$ & $\begin{array}{r}71 . \\
68 \\
43 \\
37 \\
122\end{array}$ & $\begin{array}{l}.18 \\
.23 \\
.13 \\
.01 \\
.22\end{array}$ & $\begin{array}{l}54 . \\
46 . \\
48 . \\
47 \circ^{\circ} \\
48 .\end{array}$ & $\begin{array}{l}64 . \\
72 . \\
72 . \\
92 . \\
76 .\end{array}$ & $\begin{array}{l}426 . \\
524 . \\
446 . \\
202^{\circ} \\
474^{\circ}\end{array}$ \\
\hline
\end{tabular}

$\begin{array}{llllllll}65 . & 37 . & 0.00 & 41 . & .08 & 43 . & 76 . & 413 . \\ 62 . & 37 . & 0.00 & 48 . & .18 & 45 . & 66 . & 518 . \\ 68 . & 36 . & 0.00 & 61 . & .15 & 45 . & 74 . & 459 . \\ 65 . & 37 . & 0.00 & 70 . & .16 & 43 . & 66 . & 518 . \\ 68 . & 34 . & 0.00 & 110 . & .18 & 44 . & 57 . & 547 .\end{array}$

$\begin{array}{rrrrrrrr}61 . & 35 . & 0.00 & 47 . & .28 & 44 . & 55 . & 553 . \\ 66 . & 35 . & 0.00 & 57 . & .20 & 47 . & 62 . & 465 . \\ 66 . & 35 . & 0.00 & 89 . & .28 & 50 . & 56 . & 546 . \\ 67 . & 37 . & 0.00 & 62 . & .19 & 46 . & 57 . & 513 . \\ 67 . & 35 . & 0.00 & 111 . & .28 & 46 . & 63 . & 511 .\end{array}$

$\begin{array}{llllllll}61 . & 34 . & 0.00 & 113 . & .19 & 40 . & 61 . & 477 . \\ 55 . & 35 . & 0.00 & 88 . & .25 & 51 . & 38 . & 503 . \\ 61 . & 35 . & 0.00 & 82 . & .28 & 47 . & 57 . & 497 . \\ 64 . & 36 . & 0.00 & 98 . & .22 & 46 . & 62 . & 416 . \\ 63 . & 36 . & 0.00 & 46 . & .20 & 47 . & 63 . & 439 .\end{array}$


Table 4.--Climatic data, Jefferson station,

\begin{tabular}{|c|c|c|c|c|c|c|c|}
\hline $\begin{array}{r}\text { DAY } \\
\text { OF } \\
\text { YEAR }\end{array}$ & MONTH & DAY & YEAR & $\begin{array}{l}\text { IEMPI } \\
\text { MAX } \\
\text { (F) }\end{array}$ & $\begin{array}{l}\text { TURE } \\
\text { MIN } \\
\text { (F) }\end{array}$ & $\begin{array}{l}\text { DRY } \\
\text { BULB } \\
\text { (F) }\end{array}$ & $\begin{array}{l}\text { WET } \\
\text { BULB } \\
\text { (F) }\end{array}$ \\
\hline $\begin{array}{l}271 \\
272 \\
273 \\
274 \\
121\end{array}$ & $\begin{array}{r}9 \\
9 \\
9 \\
10 \\
5\end{array}$ & $\begin{array}{r}28 \\
29 \\
30 \\
1 \\
1\end{array}$ & $\begin{array}{l}77 \\
17 \\
17 \\
77 \\
18\end{array}$ & $\begin{array}{l}62 . \\
67 . \\
68 . \\
38 . \\
43 .\end{array}$ & $\begin{array}{l}26 . \\
21 . \\
27 . \\
21 . \\
16 .\end{array}$ & $\begin{array}{l}350^{\circ} \\
380^{\circ} \\
34{ }^{\circ} \\
23{ }^{\circ} \\
30\end{array}$ & $\begin{array}{l}31 . \\
33 . \\
31 . \\
23 . \\
30\end{array}$ \\
\hline $\begin{array}{l}122 \\
123 \\
124 \\
125 \\
126\end{array}$ & $\begin{array}{l}5 \\
5 \\
5 \\
5 \\
5\end{array}$ & $\begin{array}{l}2 \\
3 \\
4 \\
5 \\
6\end{array}$ & $\begin{array}{l}78 \\
78 \\
78 \\
78 \\
78\end{array}$ & $\begin{array}{l}39 . \\
41 . \\
45 \\
45 \\
37\end{array}$ & $\begin{array}{l}22 . \\
21 . \\
20 \\
16 . \\
20 .\end{array}$ & $\begin{array}{l}27 \\
34 \\
30 \\
24 \\
\end{array}$ & $\begin{array}{l}27 \\
340^{\circ} \\
30 \\
24 \\
4\end{array}$ \\
\hline $\begin{array}{l}127 \\
128 \\
129 \\
130 \\
131\end{array}$ & $\begin{array}{l}5 \\
5 \\
5 \\
5 \\
5\end{array}$ & $\begin{array}{r}7 \\
8 \\
9 \\
10 \\
11\end{array}$ & $\begin{array}{l}78 \\
78 \\
78 \\
78 \\
78\end{array}$ & $\begin{array}{l}33 . \\
350^{\circ} \\
36 . \\
530 \\
49 .\end{array}$ & $\begin{array}{l}-5 \\
18 \\
18 \\
18 \\
23 \\
\end{array}$ & $\begin{array}{l}13 \\
26 \\
28 \\
32 \\
\end{array}$ & $\begin{array}{l}13 . \\
26 \\
28 \\
29 \\
*\end{array}$ \\
\hline $\begin{array}{l}132 \\
133 \\
134 \\
135 \\
136\end{array}$ & $\begin{array}{l}5 \\
5 \\
5 \\
5 \\
5\end{array}$ & $\begin{array}{l}12 \\
13 \\
14 \\
15 \\
16\end{array}$ & $\begin{array}{l}78 \\
78 \\
78 \\
78 \\
78\end{array}$ & 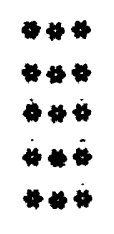 & 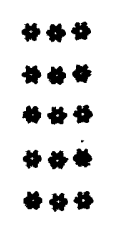 & $\begin{array}{l}* * * \\
* * * \\
* * * \\
* * * \\
* * *\end{array}$ & $\begin{array}{l}* * * \\
* * * \\
* * * \\
* * *\end{array}$ \\
\hline $\begin{array}{l}137 \\
138 \\
139 \\
140 \\
141\end{array}$ & $\begin{array}{l}5 \\
5 \\
5 \\
5 \\
5\end{array}$ & $\begin{array}{l}17 \\
18 \\
19 \\
20 \\
21\end{array}$ & $\begin{array}{l}78 \\
78 \\
78 \\
78 \\
78\end{array}$ & $\begin{array}{l}* * * \\
* * * \\
* * * \\
* * *\end{array}$ & $\begin{array}{l}\# * * \\
* * * \\
* * 4 \\
* * 4 \\
* * *\end{array}$ & $\begin{array}{l}* * * \\
* * * \\
* * * \\
* * * \\
* * *\end{array}$ & $\begin{array}{l}* * * \\
* * * \\
* * * \\
* * *\end{array}$ \\
\hline
\end{tabular}


May-September 1977, 1978, and 1979--Continued

\begin{tabular}{|c|c|c|c|c|c|c|c|}
\hline $\begin{array}{l}\text { PAN } \\
\text { MAX } \\
\text { (F) }\end{array}$ & $\begin{array}{l}\text { WATER } \\
\text { MIN } \\
\text { (F) }\end{array}$ & $\begin{array}{l}\text { PRECIP } \\
(I N \cdot)\end{array}$ & $\begin{array}{l}\text { WIND } \\
\text { (MI./DAY) }\end{array}$ & $\begin{array}{l}\text { PAN } \\
\text { EVAP } \\
(I N \cdot)\end{array}$ & $\begin{array}{l}\text { DAI } \\
\text { TEMP } \\
\text { (F) }\end{array}$ & $\begin{array}{l}\text { Y AVG } \\
\text { REL HUM } \\
\text { (PERCENT) }\end{array}$ & $\begin{array}{l}\text { SOLAR } \\
\text { RAD } \\
(L Y .)\end{array}$ \\
\hline $\begin{array}{l}67 . \\
66 . \\
63 . \\
54 . \\
53 .\end{array}$ & $\begin{array}{l}36 \\
36 . \\
35 \\
32 \\
36\end{array}$ & $\begin{array}{l}0.00 \\
0.00 \\
0.00 \\
0.00 \\
0.00\end{array}$ & $\begin{array}{l}66 . \\
53 . \\
92 . \\
72 . \\
70\end{array}$ & $\begin{array}{l}.12 \\
.29 \\
.05 \\
.39 \\
.17\end{array}$ & $\begin{array}{l}48 . \\
47 \circ^{\circ} \\
41 . \\
37 \\
31 .\end{array}$ & $\begin{array}{l}70 \\
62 \\
71 \\
64 \\
80\end{array}$ & $\begin{array}{l}436 . \\
400^{\circ} \\
314^{\circ} \\
464^{\circ} \\
384^{\circ}\end{array}$ \\
\hline $\begin{array}{l}51 . \\
51 . \\
064 \\
010\end{array}$ & $\begin{array}{l}32 . \\
35 . \\
* \$ 4 \\
+*\end{array}$ & $\begin{array}{r}.05 \\
.10 \\
.12 \\
0.00 \\
0.00\end{array}$ & $\begin{array}{r}57 . \\
155 . \\
81 . \\
103 . \\
04 .\end{array}$ & $\begin{array}{l}.16 \\
.05 \\
* 44 \\
* 4 * \\
.4 \%\end{array}$ & $\begin{array}{l}32 . \\
36 . \\
28 . \\
28 . \\
23 .\end{array}$ & $\begin{array}{l}71 . \\
68 . \\
72 . \\
77 . \\
84 .\end{array}$ & $\begin{array}{l}472 . \\
576 . \\
536 . \\
314 \\
102^{\circ}\end{array}$ \\
\hline $\begin{array}{l}* 40 \\
460 \\
440 \\
530 \\
060\end{array}$ & $\begin{array}{l}* * * \\
* * 4 \\
* * 4 \\
32 \\
* *\end{array}$ & $\begin{array}{r}.13 \\
0.00 \\
0.00 \\
.02 \\
\$ \$ \$ 4\end{array}$ & $\begin{array}{l}149 . \\
161 . \\
68 \\
* \$ 4 \\
* \$ 4\end{array}$ & 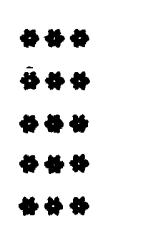 & $\begin{array}{r}22 . \\
23 . \\
+* * * \\
+* * * \\
+* * *\end{array}$ & $\begin{array}{r}68 . \\
8 \overrightarrow{1} . \\
+40 \\
040\end{array}$ & $\begin{array}{l}500 . \\
661 . \\
+* 4 * \\
* \$ 4\end{array}$ \\
\hline 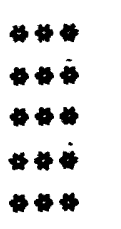 & 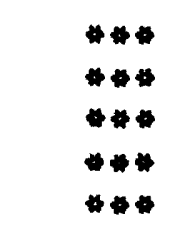 & 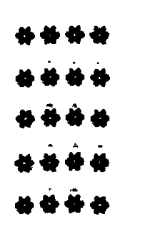 & 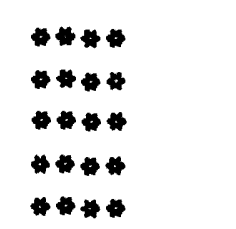 & 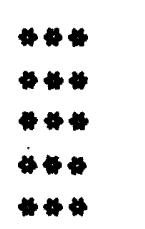 & 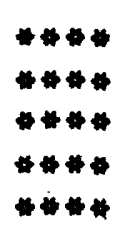 & 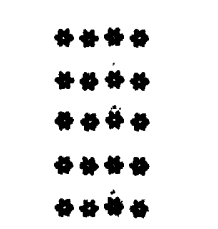 & 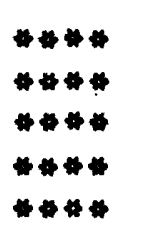 \\
\hline 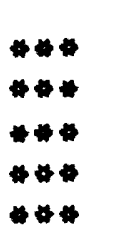 & 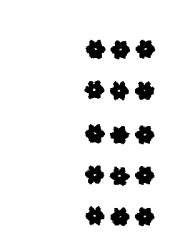 & 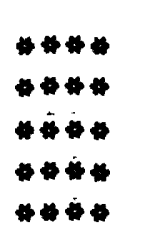 & 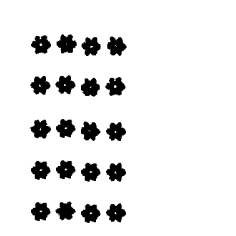 & 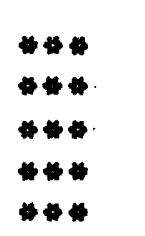 & 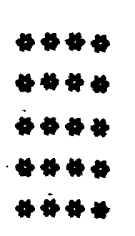 & 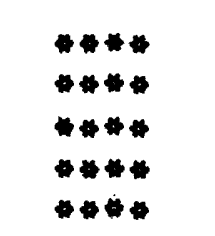 & $\begin{array}{l}* * * * \\
* * * * \\
* * * 4 \\
* * * 4 \\
* * * *\end{array}$ \\
\hline
\end{tabular}


Table 4.--Climatic data, Jefferson station,

\begin{tabular}{|c|c|c|c|c|c|c|c|}
\hline $\begin{array}{r}\text { OAY } \\
\text { OF } \\
\text { YEAR }\end{array}$ & MONTH & DAY & YEAR & $\begin{array}{l}\text { TEMF } \\
\hat{M A X} \\
(F)\end{array}$ & $\begin{array}{l}\text { TURE } \\
\text { MIN } \\
\text { (F) }\end{array}$ & $\begin{array}{l}\text { DRY } \\
\text { BULB } \\
(F)\end{array}$ & $\begin{array}{l}\text { WET } \\
\text { BULB } \\
(F)\end{array}$ \\
\hline $\begin{array}{l}142 \\
143 \\
144 \\
145 \\
146\end{array}$ & $\begin{array}{l}5 \\
5 \\
5 \\
5 \\
5\end{array}$ & $\begin{array}{l}22 \\
23 \\
24 \\
25 \\
26\end{array}$ & $\begin{array}{l}78 \\
78 \\
78 \\
78 \\
78\end{array}$ & $\begin{array}{l}* * * \\
* * * \\
7 * \\
70 . \\
62 .\end{array}$ & $\begin{array}{l}* * * \\
* * * \\
* * 4 \\
20 . \\
22 .\end{array}$ & $\begin{array}{l}* 40 \\
* 4 \\
* 4 \\
53 . \\
44 .\end{array}$ & $\begin{array}{l}* 4 * \\
4 * 4 \\
4 \% \\
40 . \\
39 .\end{array}$ \\
\hline $\begin{array}{l}147 \\
148 \\
149 \\
150 \\
151\end{array}$ & $\begin{array}{l}5 \\
5 \\
5 \\
5 \\
5\end{array}$ & $\begin{array}{l}27 \\
28 \\
29 \\
30 \\
31\end{array}$ & $\begin{array}{l}78 \\
78 \\
78 \\
78 \\
78\end{array}$ & $\begin{array}{l}62 . \\
48 . \\
48 . \\
60 . \\
64 .\end{array}$ & $\begin{array}{l}30 . \\
32 . \\
22 . \\
28 . \\
32 .\end{array}$ & $\begin{array}{l}39 . \\
39 . \\
41 . \\
47 . \\
38 .\end{array}$ & $\begin{array}{l}39 . \\
37{ }^{\circ} \\
36 . \\
41 . \\
37 .\end{array}$ \\
\hline $\begin{array}{l}152 \\
153 \\
154 \\
155 \\
156\end{array}$ & $\begin{array}{l}6 \\
6 \\
6 \\
6 \\
6\end{array}$ & $\begin{array}{l}1 \\
2 \\
3 \\
4 \\
5\end{array}$ & $\begin{array}{l}78 \\
78 \\
78 \\
78 \\
78\end{array}$ & $\begin{array}{l}60 . \\
55 . \\
61 . \\
63 . \\
49 .\end{array}$ & $\begin{array}{l}35 . \\
35 . \\
33 . \\
25 \\
31 .\end{array}$ & $\begin{array}{l}38 . \\
39 . \\
39 . \\
39 . \\
35 .\end{array}$ & $\begin{array}{l}38 . \\
39 . \\
39 . \\
38 . \\
35 .\end{array}$ \\
\hline $\begin{array}{l}157 \\
158 \\
159 \\
160 \\
161\end{array}$ & $\begin{array}{l}6 \\
6 \\
6 \\
6 \\
6\end{array}$ & $\begin{array}{r}6 \\
7 \\
8 \\
9 \\
10\end{array}$ & $\begin{array}{l}78 \\
78 \\
78 \\
78 \\
78\end{array}$ & $\begin{array}{l}49 . \\
58 . \\
58 . \\
60 . \\
69 .\end{array}$ & $\begin{array}{l}23 . \\
35 . \\
27 \\
28 \\
33 .\end{array}$ & $\begin{array}{l}41 . \\
50 . \\
40 . \\
450^{\circ} \\
49 .\end{array}$ & $\begin{array}{l}37 . \\
45 . \\
39 . \\
41 . \\
42 .\end{array}$ \\
\hline $\begin{array}{l}162 \\
163 \\
164 \\
165 \\
166\end{array}$ & $\begin{array}{l}6 \\
6 \\
6 \\
6 \\
6\end{array}$ & $\begin{array}{l}11 \\
12 \\
13 \\
14 \\
15\end{array}$ & $\begin{array}{l}18 \\
78 \\
78 \\
78 \\
78\end{array}$ & $\begin{array}{l}70 . \\
65 . \\
70 . \\
70 . \\
71 .\end{array}$ & $\begin{array}{l}35 . \\
32 . \\
29 . \\
30 . \\
30 .\end{array}$ & $\begin{array}{l}56 . \\
45 . \\
48 . \\
50 \\
42 .\end{array}$ & $\begin{array}{l}43 . \\
45 . \\
41 . \\
38\end{array}$ \\
\hline
\end{tabular}


May-September 1977, 1978, and 1979--Continued

\begin{tabular}{|c|c|c|c|c|c|c|c|}
\hline $\begin{array}{l}\text { PAN } \\
\text { MAX } \\
\text { (F) }\end{array}$ & $\begin{array}{l}\text { WATER } \\
\text { MIN } \\
\text { (F) }\end{array}$ & $\begin{array}{l}\text { PHECIP } \\
(I N \cdot)\end{array}$ & $\begin{array}{l}\text { WIND } \\
\text { (MI./DAY) }\end{array}$ & $\begin{array}{l}\text { PAN } \\
\text { EVAP } \\
(I N \bullet)\end{array}$ & $\begin{array}{l}\text { DAI } \\
\text { TEMP } \\
\text { (F) }\end{array}$ & $\begin{array}{l}\text { AVG } \\
\text { REL HUM } \\
\text { (PERCENT) }\end{array}$ & $\begin{array}{l}\text { SOLAR } \\
\text { RAD } \\
(L Y \cdot)\end{array}$ \\
\hline$* *$ & 44 & $\Delta 4 *$ & $* 4 * 4$ & $\bullet \bullet$ & $* * *$ & $4 \$$ & $4 * *$ \\
\hline $\begin{array}{l}444 \\
444\end{array}$ & $\begin{array}{l}404 \\
4 * 4\end{array}$ & $\begin{array}{l}04 * 4 \\
44 * 4\end{array}$ & $\begin{array}{l}* 4 * 4 \\
\forall * 4 * 4\end{array}$ & $\begin{array}{l}4 * 4 \\
* 4 *\end{array}$ & $\begin{array}{l}* * * 4 \\
* * * *\end{array}$ & 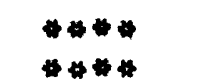 & $\begin{array}{l}4 * 4 \\
4 * 4 * 4\end{array}$ \\
\hline $\begin{array}{l}73 . \\
61\end{array}$ & $\begin{array}{l}30 \\
32 .\end{array}$ & $\begin{array}{l}* * * * \\
0.00\end{array}$ & $\begin{array}{l}102 . \\
98\end{array}$ & $\begin{array}{r}.16 \\
.38\end{array}$ & $\begin{array}{l}* 4 * \\
* * 4\end{array}$ & $\begin{array}{l}* 4 * \\
* * 4 *\end{array}$ & $\begin{array}{l}* * * 4 \\
* * * *\end{array}$ \\
\hline $\begin{array}{l}65 . \\
52 . \\
56 . \\
69 . \\
66 .\end{array}$ & $\begin{array}{l}37 \\
36 . \\
37 \\
36 . \\
38 .\end{array}$ & $\begin{array}{l}.06 \\
.07 \\
0.00 \\
0.00 \\
0.00\end{array}$ & $\begin{array}{l}91 . \\
88 . \\
128 . \\
114 . \\
103 .\end{array}$ & $\begin{array}{r}.07 \\
.11 \\
.34 \\
.38 \\
.21\end{array}$ & $\begin{array}{l}4 * 4 \\
64 \% \\
52 . \\
50 \\
45 .\end{array}$ & $\begin{array}{l}44 \% \\
64 \% \\
38 \\
48 . \\
68\end{array}$ & $\begin{array}{l}* 4 \$ 4 \\
* 44 \\
834 . \\
778 . \\
588 .\end{array}$ \\
\hline $\begin{array}{l}63 . \\
610^{\circ} \\
65^{\circ} \\
66 . \\
47 .\end{array}$ & $\begin{array}{l}38 . \\
39 . \\
39 . \\
38 . \\
36 .\end{array}$ & $\begin{array}{r}.08 \\
0.00 \\
.05 \\
0.00 \\
.02\end{array}$ & $\begin{array}{l}97 . \\
57 . \\
75 . \\
45 . \\
52 .\end{array}$ & $\begin{array}{l}.10 \\
.13 \\
.19 \\
.00 \\
.31\end{array}$ & $\begin{array}{l}43 . \\
45 . \\
47 . \\
38 . \\
40 .\end{array}$ & $\begin{array}{l}82 . \\
79 \\
70 \\
89 \\
82 .\end{array}$ & $\begin{array}{l}435 . \\
510^{\circ} \\
517^{\circ} \\
204 . \\
405^{\circ}\end{array}$ \\
\hline $\begin{array}{l}55 . \\
68 \\
61 . \\
71 . \\
72\end{array}$ & $\begin{array}{l}35 \\
38 \\
37 \\
37 \\
38 .\end{array}$ & $\begin{array}{r}.25 \\
0.00 \\
.31 \\
0.00 \\
0.00\end{array}$ & $\begin{array}{r}126 . \\
83 . \\
81 . \\
83 . \\
159 .\end{array}$ & $\begin{array}{r}.29 \\
.18 \\
.25 \\
.29 \\
.43\end{array}$ & $\begin{array}{l}45 . \\
45 . \\
46 . \\
52 . \\
55 .\end{array}$ & $\begin{array}{l}63 \\
70 \\
60 \\
60 \\
48\end{array}$ & $\begin{array}{l}828 . \\
468 . \\
783 . \\
711^{\circ} \\
744^{\circ}\end{array}$ \\
\hline $\begin{array}{l}68 . \\
73 . \\
75^{\circ} \\
77 . \\
77 .\end{array}$ & $\begin{array}{l}39 . \\
40 . \\
41 . \\
39 . \\
39 .\end{array}$ & $\begin{array}{l}0.00 \\
0.00 \\
0.00 \\
0.00 \\
0.00\end{array}$ & $\begin{array}{r}102 . \\
76 \\
67 \\
71 \\
134\end{array}$ & $\begin{array}{r}.37 \\
.29 \\
.29 \\
.32 \\
.43\end{array}$ & $\begin{array}{l}55 . \\
55 . \\
54 . \\
54 . \\
56 .\end{array}$ & $\begin{array}{l}38 . \\
62 . \\
56 . \\
49 . \\
54 .\end{array}$ & $\begin{array}{l}799 . \\
697^{\circ} \\
808 . \\
768 . \\
821 .\end{array}$ \\
\hline
\end{tabular}


Table 4.--Climatic data, Jefferson station,

\begin{tabular}{|c|c|c|c|c|c|c|c|}
\hline $\begin{array}{l}\text { DAY } \\
\text { OF } \\
\text { YEAR }\end{array}$ & MONTH & DAY & YEAR & $\begin{array}{l}\text { IEMP } \\
\text { MAX } \\
\text { (F) }\end{array}$ & $\begin{array}{l}\text { TURE } \\
\text { MIN } \\
\text { (F) }\end{array}$ & $\begin{array}{l}\text { DRY } \\
\text { BULB } \\
\text { (F) }\end{array}$ & $\begin{array}{l}\text { WET } \\
\text { BULB } \\
\text { (F) }\end{array}$ \\
\hline $\begin{array}{l}167 \\
168 \\
169 \\
170 \\
171\end{array}$ & $\begin{array}{l}6 \\
6 \\
6 \\
6 \\
6\end{array}$ & $\begin{array}{l}16 \\
17 \\
18 \\
19 \\
20\end{array}$ & $\begin{array}{l}78 \\
78 \\
78 \\
78 \\
78\end{array}$ & $\begin{array}{l}11 . \\
70 . \\
64 . \\
68 . \\
68 .\end{array}$ & $\begin{array}{l}28 . \\
30 . \\
27 . \\
30 . \\
25 .\end{array}$ & $\begin{array}{l}410^{\circ} \\
470^{\circ} \\
430^{\circ} \\
34 \circ^{\circ}\end{array}$ & $\begin{array}{l}34 . \\
39 . \\
39 . \\
37 \\
32\end{array}$ \\
\hline $\begin{array}{l}172 \\
173 \\
174 \\
175 \\
176\end{array}$ & $\begin{array}{l}6 \\
6 \\
6 \\
6 \\
6\end{array}$ & $\begin{array}{l}21 \\
22 \\
23 \\
24 \\
25\end{array}$ & $\begin{array}{l}78 \\
78 \\
78 \\
78 \\
78\end{array}$ & $\begin{array}{l}68 \\
72 \\
75 \\
15 \\
75 \\
75\end{array}$ & $\begin{array}{l}31 . \\
31 . \\
33 . \\
35 . \\
36 .\end{array}$ & $\begin{array}{l}39 . \\
41 . \\
43 . \\
52 . \\
50 .\end{array}$ & $\begin{array}{l}37 \\
36 \\
37 \\
44 \\
41\end{array}$ \\
\hline $\begin{array}{l}177 \\
178 \\
179 \\
180 \\
181\end{array}$ & $\begin{array}{l}6 \\
6 \\
6 \\
6 \\
6\end{array}$ & $\begin{array}{l}26 \\
27 \\
28 \\
29 \\
30\end{array}$ & $\begin{array}{l}78 \\
78 \\
78 \\
78 \\
78\end{array}$ & $\begin{array}{l}69 . \\
69 . \\
63 . \\
10 . \\
65 .\end{array}$ & $\begin{array}{l}30 \\
37 \\
40 \\
40 \\
43\end{array}$ & $\begin{array}{l}41 . \\
48 . \\
43 . \\
50 \\
49 .\end{array}$ & $\begin{array}{l}37 \\
460^{\circ} \\
40 \\
47 \\
47^{\circ}\end{array}$ \\
\hline $\begin{array}{l}182 \\
183 \\
184 \\
185 \\
186\end{array}$ & $\begin{array}{l}7 \\
7 \\
7 \\
7 \\
7\end{array}$ & $\begin{array}{l}1 \\
2 \\
3 \\
4 \\
5\end{array}$ & $\begin{array}{l}78 \\
78 \\
78 \\
78 \\
78\end{array}$ & $\begin{array}{l}67 . \\
10 . \\
75 \\
13 . \\
11 .\end{array}$ & $\begin{array}{l}32 . \\
34 . \\
31 . \\
28 . \\
29 .\end{array}$ & $\begin{array}{l}50 . \\
53 . \\
55 . \\
54 . \\
59\end{array}$ & $\begin{array}{l}45 . \\
460^{\circ} \\
440^{\circ} \\
43 .\end{array}$ \\
\hline $\begin{array}{l}187 \\
188 \\
189 \\
190 \\
191\end{array}$ & $\begin{array}{l}7 \\
7 \\
7 \\
7 \\
7\end{array}$ & $\begin{array}{r}6 \\
7 \\
8 \\
9 \\
10\end{array}$ & $\begin{array}{l}78 \\
78 \\
78 \\
78 \\
78\end{array}$ & $\begin{array}{l}71 . \\
69 . \\
74 . \\
74 . \\
68 .\end{array}$ & $\begin{array}{l}28 . \\
30 . \\
33 . \\
37 . \\
39 .\end{array}$ & $\begin{array}{l}55 . \\
50 \\
53 . \\
53 . \\
45\end{array}$ & $\begin{array}{l}42 . \\
46 . \\
46 . \\
44 . \\
43 .\end{array}$ \\
\hline
\end{tabular}


May-September 1977, 1978, and 1979--Continued

\begin{tabular}{|c|c|c|c|c|c|c|c|}
\hline $\begin{array}{l}\text { PAN } \\
\text { MAX } \\
(F)\end{array}$ & $\begin{array}{l}\text { WATER } \\
\text { MIN } \\
\text { (F) }\end{array}$ & $\begin{array}{l}\text { PRECIP } \\
(I N \cdot)\end{array}$ & $\begin{array}{l}\text { WIND } \\
\text { (MI./DAY) }\end{array}$ & $\begin{array}{l}\text { PAN } \\
\text { EVAP } \\
(I N \cdot)\end{array}$ & $\begin{array}{l}\text { DAI } \\
\text { TEMP } \\
\text { (F) }\end{array}$ & $\begin{array}{l}\text { Y AVG } \\
\text { REL HUM } \\
\text { (PERCENT) }\end{array}$ & $\begin{array}{l}\text { SOLAR. } \\
\text { RAO } \\
(L Y .)\end{array}$ \\
\hline $\begin{array}{l}74 . \\
71 . \\
72 . \\
75 \\
74 .\end{array}$ & $\begin{array}{l}37 . \\
36 . \\
38 . \\
38 . \\
37 .\end{array}$ & $\begin{array}{l}0.00 \\
0.00 \\
0.00 \\
0.00 \\
0.00\end{array}$ & $\begin{array}{r}133 . \\
107 . \\
89 . \\
106 \\
77\end{array}$ & $\begin{array}{l}.43 \\
.34 \\
.31 \\
.37 \\
.32\end{array}$ & $\begin{array}{l}56 . \\
51 . \\
51 . \\
53 . \\
52 .\end{array}$ & $\begin{array}{l}39 . \\
43 . \\
58 \\
48 \\
62 .\end{array}$ & $\begin{array}{l}828 . \\
796 . \\
753 . \\
822 . \\
789 .\end{array}$ \\
\hline $\begin{array}{l}73 . \\
76 \\
80 \\
81 \\
73\end{array}$ & $\begin{array}{l}37 . \\
390^{\circ} \\
39 . \\
42^{\circ} \\
43 .\end{array}$ & $\begin{array}{l}0.00 \\
0.00 \\
0.00 \\
0.00 \\
0.00\end{array}$ & $\begin{array}{l}89 . \\
65 . \\
66 . \\
96 . \\
116 .\end{array}$ & $\begin{array}{l}.38 \\
.31 \\
.35 \\
.35 \\
.41\end{array}$ & $\begin{array}{l}55 . \\
58 . \\
60 \\
59 \\
58 .\end{array}$ & $\begin{array}{l}56 . \\
490^{\circ} \\
48 . \\
470^{\circ} \\
43 .\end{array}$ & $\begin{array}{l}805 \\
740 \\
788 \\
662 \\
818 .\end{array}$ \\
\hline $\begin{array}{l}73 . \\
73 . \\
67 \\
75 . \\
65 .\end{array}$ & $\begin{array}{l}39 . \\
39 . \\
46 . \\
45 . \\
45 .\end{array}$ & $\begin{array}{r}0.00 \\
0.00 \\
.31 \\
0.00 \\
.03\end{array}$ & $\begin{array}{l}82 \\
55 \\
49 \\
74 \\
106\end{array}$ & $\begin{array}{l}.32 \\
.18 \\
.20 \\
.12 \\
.26\end{array}$ & $\begin{array}{l}54 . \\
53 . \\
58 . \\
53 . \\
55 .\end{array}$ & $\begin{array}{l}61 . \\
79 . \\
60 . \\
86 . \\
63 .\end{array}$ & $\begin{array}{l}773 \\
464 \\
527 \\
288 \\
720\end{array}$ \\
\hline $\begin{array}{l}75 . \\
75 . \\
80 . \\
72 . \\
74 .\end{array}$ & $\begin{array}{l}40 . \\
40 . \\
39 . \\
38 . \\
39 .\end{array}$ & $\begin{array}{l}0.00 \\
0.00 \\
0.00 \\
0.00 \\
0.00\end{array}$ & $\begin{array}{l}47 . \\
66 . \\
78 . \\
86 . \\
59 .\end{array}$ & $\begin{array}{r}.23 \\
.31 \\
.29 \\
.34 \\
.30\end{array}$ & $\begin{array}{l}55 . \\
58 \\
54 \\
54 \\
55 .\end{array}$ & $\begin{array}{l}57 . \\
54 . \\
50 . \\
49 . \\
51 .\end{array}$ & $\begin{array}{l}631 . \\
700 . \\
648 . \\
851 . \\
683 .\end{array}$ \\
\hline $\begin{array}{l}75 . \\
75 . \\
81 . \\
71 . \\
65 .\end{array}$ & $\begin{array}{l}39 . \\
39 . \\
43 . \\
42 . \\
42 .\end{array}$ & $\begin{array}{l}0.00 \\
0.00 \\
0.00 \\
0.00 \\
.06\end{array}$ & $\begin{array}{r}61 . \\
54 \\
104 \\
55 \\
53 .\end{array}$ & $\begin{array}{l}.25 \\
.30 \\
.39 \\
.17 \\
.16\end{array}$ & $\begin{array}{l}54 . \\
57 . \\
59 . \\
53 . \\
54 .\end{array}$ & $\begin{array}{l}62 . \\
60 . \\
52 . \\
67 . \\
75 .\end{array}$ & $\begin{array}{l}736 . \\
766 . \\
783 \\
405 \\
488\end{array}$ \\
\hline
\end{tabular}


Table 4.--Climatic data, Jefferson station,

\begin{tabular}{|c|c|c|c|c|c|c|c|}
\hline $\begin{array}{r}\text { DAY } \\
\text { OF } \\
\text { YEAR }\end{array}$ & MONTH & DAY & YEAR & $\begin{array}{l}\text { IEMPE } \\
\text { MAX } \\
\text { (F) }\end{array}$ & $\begin{array}{l}\text { TURE } \\
\text { MIN } \\
\text { (F) }\end{array}$ & $\begin{array}{l}\text { DRY } \\
\text { BULB } \\
\text { (F) }\end{array}$ & $\begin{array}{l}\text { WET } \\
\text { BULB } \\
\text { (F) }\end{array}$ \\
\hline $\begin{array}{l}217 \\
218 \\
219 \\
220 \\
221\end{array}$ & $\begin{array}{l}8 \\
8 \\
8 \\
8 \\
8\end{array}$ & $\begin{array}{l}5 \\
6 \\
7 \\
8 \\
9\end{array}$ & $\begin{array}{l}78 \\
78 \\
78 \\
78 \\
78\end{array}$ & $\begin{array}{l}11 . \\
10 . \\
10 . \\
11 . \\
66 .\end{array}$ & $\begin{array}{l}33 \\
35 \\
34 \\
32 \\
32 \\
\end{array}$ & $\begin{array}{l}49 . \\
47 \\
48 \\
53 \\
52\end{array}$ & $\begin{array}{l}42 \\
40 \\
40 \\
47 \\
48\end{array}$ \\
\hline $\begin{array}{l}222 \\
223 \\
224 \\
225 \\
226\end{array}$ & $\begin{array}{l}8 \\
8 \\
8 \\
8 \\
8\end{array}$ & $\begin{array}{l}10 \\
11 \\
12 \\
13 \\
14\end{array}$ & $\begin{array}{l}78 \\
78 \\
78 \\
78 \\
78\end{array}$ & $\begin{array}{l}65 . \\
10 \\
12 \\
11 \\
10 \\
\end{array}$ & $\begin{array}{l}32 \\
31 \\
35 \\
34 \\
32\end{array}$ & $\begin{array}{l}52 . \\
450^{\circ} \\
50 \\
47 \\
46 .\end{array}$ & $\begin{array}{l}47 . \\
410^{\circ} \\
450^{\circ} \\
42 \\
42\end{array}$ \\
\hline $\begin{array}{l}227 \\
228 \\
229 \\
230 \\
231\end{array}$ & $\begin{array}{l}8 \\
8 \\
8 \\
8 \\
8\end{array}$ & $\begin{array}{l}15 \\
16 \\
17 \\
18 \\
19\end{array}$ & $\begin{array}{l}78 \\
78 \\
78 \\
78 \\
18\end{array}$ & $\begin{array}{l}65 . \\
67 . \\
11 . \\
72 . \\
02 .\end{array}$ & $\begin{array}{l}30 \\
28 . \\
26 . \\
31 . \\
22 .\end{array}$ & $\begin{array}{l}42 . \\
450^{\circ} \\
490^{\circ} \\
48 \\
41 .\end{array}$ & $\begin{array}{l}34 . \\
390^{\circ} \\
40 . \\
420^{\circ} \\
39 .\end{array}$ \\
\hline $\begin{array}{l}232 \\
233 \\
234 \\
235 \\
236\end{array}$ & $\begin{array}{l}8 \\
8 \\
8 \\
8 \\
8\end{array}$ & $\begin{array}{l}20 \\
21 \\
22 \\
23 \\
24\end{array}$ & $\begin{array}{l}78 \\
78 \\
78 \\
78 \\
78\end{array}$ & $\begin{array}{l}69 . \\
10 . \\
10 . \\
65 . \\
11 .\end{array}$ & $\begin{array}{l}290^{\circ} \\
34 \circ^{\circ} \\
330^{\circ} \\
290^{\circ} \\
34^{\circ}\end{array}$ & $\begin{array}{l}42 . \\
46 . \\
46 . \\
43 \\
42\end{array}$ & $\begin{array}{l}40 \\
41 . \\
43 \\
40 \\
37\end{array}$ \\
\hline $\begin{array}{l}237 \\
238 \\
239 \\
240 \\
241\end{array}$ & $\begin{array}{l}8 \\
8 \\
8 \\
8 \\
8\end{array}$ & $\begin{array}{l}25 \\
26 \\
27 \\
28 \\
29\end{array}$ & $\begin{array}{l}78 \\
78 \\
18 \\
78 \\
78\end{array}$ & $\begin{array}{l}68 . \\
66 . \\
10 . \\
69 . \\
69 .\end{array}$ & $\begin{array}{l}36 . \\
29 . \\
32 . \\
29 \\
30\end{array}$ & $\begin{array}{l}48 . \\
41 . \\
470 \\
45 . \\
43 .\end{array}$ & $\begin{array}{l}44 . \\
33 \\
42 \\
40 \\
41\end{array}$ \\
\hline
\end{tabular}


May-September 1977, 1978, and 1979--Continued

\begin{tabular}{|c|c|c|c|c|c|c|c|}
\hline $\begin{array}{l}\text { PAN } \\
\text { MAX } \\
\text { (F) }\end{array}$ & $\begin{array}{l}\text { WATER } \\
\text { MIN } \\
\text { (F) }\end{array}$ & $\begin{array}{l}\text { PRECIP } \\
(I N \cdot)\end{array}$ & $\begin{array}{l}\text { WIND } \\
\text { (MI./DAY) }\end{array}$ & $\begin{array}{l}\text { PAN } \\
\text { EVAP } \\
(I N \cdot)\end{array}$ & $\begin{array}{l}\text { DAI } \\
\text { TEMP } \\
\text { (F) }\end{array}$ & $\begin{array}{l}\text { Y AVG } \\
\text { REL HUM } \\
\text { (PERCENT) }\end{array}$ & $\begin{array}{l}\text { SOLAK } \\
\text { RAD } \\
(L Y .)\end{array}$ \\
\hline $\begin{array}{l}76 . \\
74 \\
79 \\
80 \\
62 .\end{array}$ & $\begin{array}{l}43 . \\
43 . \\
42^{\circ} \\
39 . \\
37 .\end{array}$ & $\begin{array}{l}.05 \\
0.00 \\
0.00 \\
0.00 \\
0.00\end{array}$ & $\begin{array}{l}41 . \\
36 . \\
27 \\
21 . \\
44 .\end{array}$ & $\begin{array}{l}.17 \\
.44 \\
.44 \\
.06 \\
.14\end{array}$ & $\begin{array}{r}52 . \\
53 . \\
544 \\
51 . \\
51 .\end{array}$ & $\begin{array}{r}53 . \\
62 . \\
61 \\
70\end{array}$ & $\begin{array}{l}746 . \\
596 . \\
413 . \\
517 . \\
494 .\end{array}$ \\
\hline $\begin{array}{l}68 . \\
67 . \\
66 . \\
68 . \\
67 .\end{array}$ & $\begin{array}{l}41 . \\
39 . \\
40 . \\
43 . \\
41 .\end{array}$ & $\begin{array}{l}0.00 \\
0.00 \\
0.00 \\
.23 \\
0.00\end{array}$ & $\begin{array}{l}66 \\
52 \\
50 \\
51 \\
127\end{array}$ & $\begin{array}{r}.20 \\
.15 \\
.17 \\
.14 \\
.26\end{array}$ & $\begin{array}{l}51 . \\
52 . \\
55 . \\
52 . \\
45 .\end{array}$ & $\begin{array}{l}63 . \\
69 \\
66 . \\
74 \\
68 .\end{array}$ & $\begin{array}{l}573 . \\
456 . \\
428 . \\
412 . \\
572 .\end{array}$ \\
\hline $\begin{array}{l}61 . \\
73 . \\
72 . \\
72 . \\
75 .\end{array}$ & $\begin{array}{l}33 . \\
32 . \\
36 . \\
39 . \\
36 .\end{array}$ & $\begin{array}{l}.04 \\
0.00 \\
0.00 \\
0.00 \\
0.00\end{array}$ & $\begin{array}{l}19 . \\
92 . \\
85 . \\
91 . \\
47 .\end{array}$ & $\begin{array}{l}.25 \\
.23 \\
.43 \\
.24 \\
.17\end{array}$ & $\begin{array}{l}47 . \\
510^{\circ} \\
55 \circ \\
470^{\circ} \\
41 .\end{array}$ & $\begin{array}{l}42 \\
46 . \\
36 \\
60 \\
64 .\end{array}$ & $\begin{array}{l}726 . \\
723 . \\
734 . \\
619 . \\
556 .\end{array}$ \\
\hline $\begin{array}{l}67 . \\
70 \\
71 . \\
68 \\
63 .\end{array}$ & $\begin{array}{l}35 . \\
40 \\
42 . \\
40 \\
39\end{array}$ & $\begin{array}{l}0.00 \\
0.00 \\
0.00 \\
0.00 \\
0.00\end{array}$ & $\begin{array}{l}38 . \\
42 . \\
59 \\
48 \\
49\end{array}$ & $\begin{array}{l}.19 \\
.16 \\
.18 \\
.22 \\
.20\end{array}$ & $\begin{array}{l}4 * 4 \\
4 * 4 \\
52 . \\
52 . \\
54 .\end{array}$ & $\begin{array}{l}444 \\
44 \\
68 . \\
60 \\
58 .\end{array}$ & $\begin{array}{l}514^{\circ} \\
384^{\circ} \\
431^{\circ} \\
579 . \\
425 .\end{array}$ \\
\hline $\begin{array}{l}67 . \\
710^{\circ} \\
71 . \\
68 . \\
74 \circ\end{array}$ & $\begin{array}{l}42 . \\
39 . \\
39 \\
38 \\
41 .\end{array}$ & $\begin{array}{l}0.00 \\
0.000 \\
0.00 \\
0.00 \\
0.00\end{array}$ & $\begin{array}{l}72 . \\
44 . \\
55 . \\
51 . \\
36 .\end{array}$ & $\begin{array}{l}.17 \\
.19 \\
.22 \\
.20 \\
.10\end{array}$ & $\begin{array}{l}51 . \\
51 . \\
52 . \\
49 . \\
47 .\end{array}$ & $\begin{array}{l}70 \\
54 \\
43 . \\
64 . \\
74 .\end{array}$ & $\begin{array}{l}491 . \\
528 . \\
518 . \\
541 . \\
383 .\end{array}$ \\
\hline
\end{tabular}


Table 4.--Climatic data, Jefferson station,

\begin{tabular}{|c|c|c|c|c|c|c|c|}
\hline $\begin{array}{r}\text { DAY } \\
\text { OF } \\
\text { YEAR }\end{array}$ & MONTH & DAY & YEAR & $\begin{array}{l}\text { TEMP } \\
\text { MAX } \\
\text { (F) }\end{array}$ & $\begin{array}{l}\text { URE } \\
\text { MIN } \\
\text { (F) }\end{array}$ & $\begin{array}{l}\text { DRY } \\
\text { BULB } \\
(F)\end{array}$ & $\begin{array}{l}\text { WET } \\
\text { BULB } \\
\text { (F) }\end{array}$ \\
\hline $\begin{array}{l}192 \\
193 \\
194 \\
195 \\
196\end{array}$ & $\begin{array}{l}7 \\
7 \\
7 \\
7 \\
7\end{array}$ & $\begin{array}{l}11 \\
12 \\
13 \\
14 \\
15\end{array}$ & $\begin{array}{l}78 \\
78 \\
78 \\
78 \\
78\end{array}$ & $\begin{array}{l}69 . \\
100 \\
130 \\
15 . \\
77 .\end{array}$ & $\begin{array}{l}40 . \\
35 \\
36 \\
34 . \\
39 .\end{array}$ & $\begin{array}{l}49 . \\
53 . \\
51 . \\
53 . \\
52 .\end{array}$ & $\begin{array}{l}43{ }^{\circ} \\
490^{\circ} \\
49^{\circ} \\
49^{\circ}\end{array}$ \\
\hline $\begin{array}{l}197 \\
198 \\
199 \\
200 \\
201\end{array}$ & $\begin{array}{l}7 \\
7 \\
7 \\
7 \\
7\end{array}$ & $\begin{array}{l}16 \\
17 \\
18 \\
19 \\
20\end{array}$ & $\begin{array}{l}78 \\
78 \\
78 \\
78 \\
78\end{array}$ & $\begin{array}{l}13 . \\
75 \\
71 \\
14 \\
11 .\end{array}$ & $\begin{array}{l}35 . \\
43 \\
39 \\
36 \\
35\end{array}$ & $\begin{array}{l}54 . \\
550^{\circ} \\
470^{\circ} \\
550^{\circ} \\
54 .\end{array}$ & $\begin{array}{l}48 \\
48 \\
42 \\
47 \\
48\end{array}$ \\
\hline $\begin{array}{l}202 \\
203 \\
204 \\
205 \\
206\end{array}$ & $\begin{array}{l}7 \\
7 \\
7 \\
7 \\
7\end{array}$ & $\begin{array}{l}21 \\
22 \\
23 \\
24 \\
25\end{array}$ & $\begin{array}{l}78 \\
78 \\
78 \\
78 \\
78\end{array}$ & $\begin{array}{l}69 . \\
730 \\
70 . \\
72 . \\
76 .\end{array}$ & $\begin{array}{l}34{ }^{\circ} \\
32{ }^{\circ} \\
410^{\circ} \\
35{ }^{\circ} \\
34\end{array}$ & $\begin{array}{l}49 . \\
48 . \\
51 . \\
49 . \\
55 .\end{array}$ & $\begin{array}{l}46 . \\
43{ }^{\circ} \\
480^{\circ} \\
44{ }^{\circ}\end{array}$ \\
\hline $\begin{array}{l}207 \\
208 \\
209 \\
210 \\
211\end{array}$ & $\begin{array}{l}7 \\
7 \\
7 \\
7 \\
7\end{array}$ & $\begin{array}{l}26 \\
27 \\
28 \\
29 \\
30\end{array}$ & $\begin{array}{l}78 \\
78 \\
78 \\
78 \\
78\end{array}$ & $\begin{array}{l}75 . \\
75 . \\
77 \\
16 . \\
10\end{array}$ & $\begin{array}{l}34{ }^{\circ} \\
360^{\circ} \\
340^{\circ} \\
390^{\circ} \\
35{ }^{\circ}\end{array}$ & $\begin{array}{l}52 . \\
55 . \\
52 . \\
57 . \\
50\end{array}$ & $\begin{array}{l}47 \\
490^{\circ} \\
460^{\circ} \\
49\end{array}$ \\
\hline $\begin{array}{l}212 \\
213 \\
214 \\
215 \\
216\end{array}$ & $\begin{array}{l}7 \\
8 \\
8 \\
8 \\
8\end{array}$ & $\begin{array}{r}31 \\
1 \\
2 \\
3 \\
4\end{array}$ & $\begin{array}{l}78 \\
78 \\
78 \\
78 \\
78\end{array}$ & $\begin{array}{l}11 . \\
610 \\
100 \\
71 . \\
69 .\end{array}$ & $\begin{array}{l}37{ }^{\circ} \\
34 \circ^{\circ} \\
33{ }^{\circ} \\
33{ }^{\circ} \\
30^{\circ}\end{array}$ & $\begin{array}{l}49 . \\
500^{\circ} \\
490^{\circ} \\
470^{\circ}\end{array}$ & $\begin{array}{l}470^{\circ} \\
460^{\circ} \\
420^{\circ} \\
450^{\circ} \\
43\end{array}$ \\
\hline
\end{tabular}


May-September 1977, 1978, and 1979--Continued

\begin{tabular}{|c|c|c|c|c|c|c|c|}
\hline $\begin{array}{l}\text { PAN } \\
\text { MAX } \\
(F)\end{array}$ & $\begin{array}{l}\text { WATER } \\
\text { MIN } \\
\text { (F) }\end{array}$ & $\begin{array}{l}\text { PRECIP } \\
(I N \cdot)\end{array}$ & $\begin{array}{l}\text { WIND } \\
\text { (MI•/DAY) }\end{array}$ & $\begin{array}{l}\text { PAN } \\
\text { EVAP } \\
(I N .)\end{array}$ & $\begin{array}{l}\text { DAI } \\
\text { TEMP } \\
\text { (F) }\end{array}$ & $\begin{array}{l}\text { Y AVG } \\
\text { REL HUM } \\
\text { (PERCENT) }\end{array}$ & $\begin{array}{l}\text { SOLAR } \\
\text { RAD } \\
(L Y .)\end{array}$ \\
\hline $\begin{array}{l}73 \\
75 \\
77 \\
79 \\
80\end{array}$ & $\begin{array}{l}47^{\circ} \\
44 \circ^{\circ} \\
44 \circ^{\circ} \\
46{ }^{\circ} \\
45^{\circ}\end{array}$ & $\begin{array}{r}.05 \\
.02 \\
0.00 \\
0.00 \\
0.00\end{array}$ & $\begin{array}{l}75 . \\
61 . \\
54 . \\
56 . \\
67 .\end{array}$ & $\begin{array}{l}.23 \\
.26 \\
.29 \\
.30 \\
.14\end{array}$ & $\begin{array}{l}56 . \\
57 . \\
60 \\
61 . \\
57 .\end{array}$ & $\begin{array}{l}67 . \\
58 . \\
60 \\
5 \overline{8} \\
6 \overline{1} .\end{array}$ & $\begin{array}{l}603 . \\
713 . \\
700 \\
660 \\
373 .\end{array}$ \\
\hline $\begin{array}{l}65 . \\
72 . \\
79 . \\
80 \\
70\end{array}$ & $\begin{array}{l}42^{\circ} \\
43^{\circ} \\
46 \\
45 \\
44\end{array}$ & $\begin{array}{l}0.00 \\
0.00 \\
0.00 \\
0.00 \\
0.00\end{array}$ & $\begin{array}{l}75 \\
78 \\
51 \\
44 \\
50\end{array}$ & $\begin{array}{l}.19 \\
.25 \\
.20 \\
.25 \\
.13\end{array}$ & $\begin{array}{l}57 . \\
60 . \\
57 . \\
56 . \\
54 .\end{array}$ & $\begin{array}{l}63 . \\
52 . \\
60 \\
61 . \\
69 .\end{array}$ & $\begin{array}{l}504 . \\
622^{\circ} \\
734 \\
435^{\circ} \\
518^{\circ}\end{array}$ \\
\hline $\begin{array}{l}68 . \\
74 \\
81 . \\
83 \\
82 .\end{array}$ & $\begin{array}{l}43 \\
44{ }^{\circ} \\
44 \\
45 \\
42^{\circ}\end{array}$ & $\begin{array}{r}0.00 \\
0.00 \\
.16 \\
0.00 \\
0.00\end{array}$ & $\begin{array}{l}68 \\
45 \\
37 \\
48 \\
73\end{array}$ & $\begin{array}{r}.27 \\
.38 \\
.24 \\
.28 \\
.28\end{array}$ & $\begin{array}{l}56 . \\
52 . \\
56 . \\
55 . \\
59 .\end{array}$ & $\begin{array}{l}64 . \\
72 . \\
61 . \\
53 \\
44\end{array}$ & $\begin{array}{l}661 . \\
732 \\
685 \\
655 \\
645 .\end{array}$ \\
\hline $\begin{array}{l}80 . \\
72 . \\
80 . \\
71 . \\
70 .\end{array}$ & $\begin{array}{l}410^{\circ} \\
4 \circ^{\circ} \\
44 \circ^{\circ} \\
440^{\circ} \\
43 .\end{array}$ & $\begin{array}{r}0.00 \\
0.00 \\
0.00 \\
0.00 \\
.17\end{array}$ & $\begin{array}{l}51 . \\
45{ }^{\circ} \\
54 \\
54{ }^{\circ} \\
51 .\end{array}$ & $\begin{array}{l}.22 \\
.24 \\
.16 \\
.19 \\
.24\end{array}$ & $\begin{array}{l}57 . \\
58 . \\
56 . \\
57 . \\
55 .\end{array}$ & $\begin{array}{l}54 \\
58 \\
65 . \\
67 \\
62 .\end{array}$ & $\begin{array}{l}504 . \\
618^{\circ} \\
377 \\
511^{\circ} \\
75 !\end{array}$ \\
\hline $\begin{array}{l}78 \\
76 \\
74 \\
74 \\
75^{\circ}\end{array}$ & $\begin{array}{l}44{ }^{\circ} \\
45^{\circ} \\
410^{\circ} \\
40^{\circ} \\
42^{\circ}\end{array}$ & $\begin{array}{l}0.00 \\
0.00 \\
0.00 \\
0.00 \\
0.00\end{array}$ & $\begin{array}{l}48 . \\
59 . \\
41 . \\
22 . \\
40\end{array}$ & $\begin{array}{l}.22 \\
.23 \\
.17 \\
.16 \\
.22\end{array}$ & $\begin{array}{r}4 * 4 \\
51 . \\
53 . \\
51 . \\
51 .\end{array}$ & $\begin{array}{l}6 \% \\
63 . \\
64 . \\
82 . \\
63 .\end{array}$ & $\begin{array}{l}550 \\
569 . \\
685 . \\
546 \\
636\end{array}$ \\
\hline
\end{tabular}


Table 4.--Climatic data, Jefferson station,

\begin{tabular}{|c|c|c|c|c|c|c|c|}
\hline $\begin{array}{r}\text { DAY } \\
\text { OF } \\
\text { YEAR }\end{array}$ & MONTH. & DAY & YEAR & $\begin{array}{l}\text { TEMF } \\
\text { MAX } \\
\text { (F) }\end{array}$ & $\begin{array}{l}\text { TURE } \\
\text { MIN } \\
\text { (F) }\end{array}$ & $\begin{array}{l}\text { DRY } \\
\text { BULB } \\
\text { (F) }\end{array}$ & $\begin{array}{l}\text { WET } \\
\text { BULE } \\
\text { (F) }\end{array}$ \\
\hline $\begin{array}{l}242 \\
243 \\
244 \\
245 \\
246\end{array}$ & $\begin{array}{l}8 \\
8 \\
9 \\
9 \\
9\end{array}$ & $\begin{array}{r}30 \\
31 \\
1 \\
2 \\
3\end{array}$ & $\begin{array}{l}78 \\
78 \\
78 \\
78 \\
78\end{array}$ & $\begin{array}{l}62 . \\
68 . \\
65 . \\
69 . \\
69 .\end{array}$ & $\begin{array}{l}27 . \\
30 . \\
29 . \\
30 . \\
33 .\end{array}$ & $\begin{array}{l}44 . \\
45 . \\
40 \\
49 \\
44 .\end{array}$ & $\begin{array}{l}41 \\
40 \\
37 \\
41 \\
40\end{array}$ \\
\hline $\begin{array}{l}247 \\
248 \\
249 \\
250 \\
251\end{array}$ & $\begin{array}{l}9 \\
9 \\
9 \\
9 \\
9\end{array}$ & $\begin{array}{l}4 \\
5 \\
6 \\
7 \\
8\end{array}$ & $\begin{array}{l}78 \\
78 \\
78 \\
78 \\
78\end{array}$ & $\begin{array}{l}12 . \\
14 . \\
77 . \\
76 . \\
15 .\end{array}$ & $\begin{array}{l}34 . \\
32 . \\
31 . \\
30 . \\
29 .\end{array}$ & $\begin{array}{l}42 . \\
41 . \\
40 \\
41 . \\
30\end{array}$ & $\begin{array}{l}38 \\
34 . \\
35 \\
34 \\
29\end{array}$ \\
\hline $\begin{array}{l}252 \\
253 \\
254 \\
255 \\
256\end{array}$ & $\begin{array}{l}9 \\
9 \\
9 \\
9 \\
9\end{array}$ & $\begin{array}{r}9 \\
10 \\
11 \\
12 \\
13\end{array}$ & $\begin{array}{l}78 \\
78 \\
78 \\
78 \\
78\end{array}$ & $\begin{array}{l}11 . \\
70 . \\
68 . \\
59 . \\
60 .\end{array}$ & $\begin{array}{l}29 . \\
29 . \\
320^{\circ} \\
27 . \\
170^{\circ}\end{array}$ & $\begin{array}{l}44 \\
44 \\
40 \\
40 \\
36\end{array}$ & $\begin{array}{l}39 \\
40 \\
35 \\
33 \\
33\end{array}$ \\
\hline $\begin{array}{l}257 \\
258 \\
259 \\
260 \\
261\end{array}$ & $\begin{array}{l}9 \\
9 \\
9 \\
9 \\
9\end{array}$ & $\begin{array}{l}14 \\
15 \\
16 \\
17 \\
18\end{array}$ & $\begin{array}{l}78 \\
78 \\
78 \\
78 \\
78\end{array}$ & $\begin{array}{l}38 . \\
66 . \\
74 . \\
67 . \\
61 .\end{array}$ & $\begin{array}{l}20 . \\
26 . \\
30 . \\
32 . \\
24 .\end{array}$ & $\begin{array}{l}40 . \\
41 . \\
47 \\
39 \\
35\end{array}$ & $\begin{array}{l}35 . \\
39 . \\
43 . \\
38 \\
35\end{array}$ \\
\hline $\begin{array}{l}262 \\
263 \\
264 \\
265 \\
266\end{array}$ & $\begin{array}{l}9 \\
9 \\
9 \\
9 \\
9\end{array}$ & $\begin{array}{l}19 \\
20 \\
21 \\
22 \\
23\end{array}$ & $\begin{array}{l}78 \\
78 \\
78 \\
78 \\
78\end{array}$ & $\begin{array}{l}63 . \\
35 . \\
38 . \\
55 . \\
64 .\end{array}$ & $\begin{array}{r}25 . \\
25 . \\
3 . \\
5 . \\
19 .\end{array}$ & $\begin{array}{l}37 . \\
31: \\
4 \% \\
32 .\end{array}$ & $\begin{array}{l}34 . \\
31: \\
* * 4 \\
29 .\end{array}$ \\
\hline $\begin{array}{l}267 \\
268 \\
269 \\
270 \\
271\end{array}$ & $\begin{array}{l}9 \\
9 \\
9 \\
9 \\
9\end{array}$ & $\begin{array}{l}24 \\
25 \\
26 \\
27 \\
28\end{array}$ & $\begin{array}{l}78 \\
78 \\
78 \\
78 \\
78\end{array}$ & $\begin{array}{l}69 . \\
57 . \\
58 . \\
66 . \\
65 .\end{array}$ & $\begin{array}{l}27 . \\
31 . \\
22 . \\
24 . \\
25 .\end{array}$ & $\begin{array}{l}37 \\
35 \\
27 \\
30 \\
28 \\
\end{array}$ & $\begin{array}{l}34 . \\
330^{\circ} \\
270^{\circ} \\
29 . \\
28 .\end{array}$ \\
\hline $\begin{array}{c}272 \\
273\end{array}$ & $\begin{array}{l}9 \\
9\end{array}$ & $\begin{array}{l}.29 \\
30\end{array}$ & $\begin{array}{l}78 \\
78\end{array}$ & $\begin{array}{l}66 . \\
64 .\end{array}$ & $\begin{array}{l}28 . \\
20 .\end{array}$ & $\begin{array}{l}33 . \\
30 .\end{array}$ & $\begin{array}{l}31 . \\
30 .\end{array}$ \\
\hline
\end{tabular}


May-September 1977, 1978, and 1979--Continued

\begin{tabular}{|c|c|c|c|c|c|c|c|}
\hline $\begin{array}{l}\text { PAN } \\
\text { MAX } \\
\text { (F) }\end{array}$ & $\begin{array}{l}\text { WATER } \\
\text { MIN } \\
\text { (F) }\end{array}$ & $\begin{array}{l}\text { PRECIP } \\
(I N \cdot)\end{array}$ & $\begin{array}{l}\text { WIND } \\
\text { (MI./DAY) }\end{array}$ & $\begin{array}{l}\text { PAN } \\
\text { EVAP } \\
(I N .)\end{array}$ & $\begin{array}{l}\text { DAII } \\
\text { TEMP } \\
(F)\end{array}$ & $\begin{array}{l}\text { Y AVG } \\
\text { REL HUM } \\
\text { (PERCENT) }\end{array}$ & $\begin{array}{l}\text { SOLAR } \\
\text { RAD } \\
(L Y .)\end{array}$ \\
\hline $\begin{array}{l}64 . \\
74 . \\
62 . \\
73 \\
64 .\end{array}$ & $\begin{array}{l}39 . \\
39 . \\
37 . \\
37 . \\
38 .\end{array}$ & $\begin{array}{r}0.00 \\
0.00 \\
.13 \\
0.00 \\
0.00\end{array}$ & $\begin{array}{l}43 . \\
23 . \\
56 . \\
51 . \\
37 .\end{array}$ & $\begin{array}{l}.18 \\
.12 \\
.27 \\
.10 \\
.22\end{array}$ & $\begin{array}{l}49 . \\
45 . \\
51 . \\
51 . \\
54 .\end{array}$ & $\begin{array}{l}67 . \\
79 \\
57 \\
63 \\
55^{\circ}\end{array}$ & $\begin{array}{l}564 . \\
369 . \\
631 . \\
351 . \\
613 .\end{array}$ \\
\hline $\begin{array}{l}78 \\
76 \\
80 \\
73 \\
76\end{array}$ & $\begin{array}{l}39 . \\
39 . \\
38 . \\
38 \\
38 .\end{array}$ & $\begin{array}{r}0.00 \\
0.00 \\
0.00 \\
0.00 \\
.03\end{array}$ & $\begin{array}{l}38 \\
45 \circ \\
48 \\
53 \\
50\end{array}$ & $\begin{array}{l}.22 \\
.26 \\
.23 \\
.18 \\
.11\end{array}$ & $\begin{array}{l}54 . \\
55 . \\
54 . \\
51 . \\
52 .\end{array}$ & $\begin{array}{l}63 . \\
52 . \\
59 . \\
73 . \\
60 .\end{array}$ & $\begin{array}{l}481 . \\
603 . \\
494 . \\
510 \\
588\end{array}$ \\
\hline $\begin{array}{l}77 . \\
69 . \\
66 . \\
74 . \\
64 .\end{array}$ & $\begin{array}{l}37 \\
38 \\
37 \\
36 \\
33\end{array}$ & $\begin{array}{l}0.00 \\
0.00 \\
0.00 \\
0.00 \\
0.00\end{array}$ & $\begin{array}{r}45 . \\
70 \\
116 \\
103 \\
74\end{array}$ & $\begin{array}{l}.17 \\
.14 \\
.02 \\
.34 \\
.18\end{array}$ & $\begin{array}{l}52 . \\
490^{\circ} \\
46 . \\
43 \\
40\end{array}$ & $\begin{array}{l}63 . \\
70 \\
49 \\
61 \\
70\end{array}$ & $\begin{array}{l}419^{\circ} \\
442^{\circ} \\
562^{\circ} \\
602^{\circ} \\
513^{\circ}\end{array}$ \\
\hline $\begin{array}{l}62 . \\
68 . \\
74 . \\
71^{\circ} \\
64 .\end{array}$ & $\begin{array}{l}33 . \\
35 \\
37 \\
38 \\
36\end{array}$ & $\begin{array}{r}0.00 \\
0.00 \\
0.00 \\
.02 \\
0.00\end{array}$ & $\begin{array}{l}52 . \\
69 \\
66 \\
50 \\
135\end{array}$ & $\begin{array}{l}.20 \\
.26 \\
.20 \\
.12 \\
.23\end{array}$ & $\begin{array}{l}45 . \\
51{ }^{\circ} \\
52^{\circ} \\
46 . \\
44 .\end{array}$ & $\begin{array}{l}65 . \\
06 . \\
68 . \\
79 . \\
65 .\end{array}$ & $\begin{array}{l}517^{\circ} \\
494^{\circ} \\
485^{\circ} \\
340^{\circ} \\
469^{\circ}\end{array}$ \\
\hline $\begin{array}{l}61 . \\
464 \\
404 \\
656\end{array}$ & $\begin{array}{l}36 . \\
* * \\
+4 \\
32 .\end{array}$ & $\begin{array}{l}0.00 \\
0.00 \\
0.00 \\
.47 \\
0.00\end{array}$ & $\begin{array}{l}32 . \\
58 \\
33 \\
40 \\
46 .\end{array}$ & 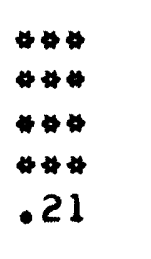 & $\begin{array}{l}31 . \\
30 \\
32 \\
42 . \\
47 .\end{array}$ & $\begin{array}{l}\$ 4 \\
90 . \\
72 . \\
71 . \\
65 .\end{array}$ & $\begin{array}{r}78 \\
285^{\circ} \\
516^{\circ} \\
523 \\
485^{\circ}\end{array}$ \\
\hline $\begin{array}{l}73 . \\
53 . \\
62 . \\
67 . \\
66^{\circ}\end{array}$ & $\begin{array}{l}35 \\
36 \\
38 \\
35 \\
36\end{array}$ & $\begin{array}{l}0.00 \\
0.00 \\
0.00 \\
0.00 \\
0.00\end{array}$ & $\begin{array}{l}46 . \\
33 . \\
60 . \\
41 . \\
63 .\end{array}$ & $\begin{array}{l}.12 \\
.11 \\
.22 \\
.15 \\
.19\end{array}$ & 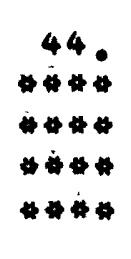 & $\begin{array}{r}79 . \\
* 4 \% \\
* 4 * 4 \\
* 4 * 4\end{array}$ & $\begin{array}{l}238 \\
337 \\
475^{\circ} \\
409 \\
413^{\circ}\end{array}$ \\
\hline $\begin{array}{l}64 . \\
64 .\end{array}$ & $\begin{array}{l}37 . \\
34 .\end{array}$ & $\begin{array}{l}0.00 \\
0.00\end{array}$ & $\begin{array}{l}73 . \\
57 .\end{array}$ & $\begin{array}{l}.21 \\
.19\end{array}$ & $\begin{array}{l}\star \star \star \star \\
\star \star \star \star\end{array}$ & $\begin{array}{l}\star \star \star \star \\
\star \star \star \star\end{array}$ & $\begin{array}{l}446 . \\
435 .\end{array}$ \\
\hline
\end{tabular}


Table 4.---Climatic data, Jefferson station,

\begin{tabular}{|c|c|c|c|c|c|c|}
\hline $\begin{array}{r}\text { DAY } \\
\text { OF } \\
\text { YEAR }\end{array}$ & MONTH & DAY & YEAR & $\begin{array}{c}\text { TEMP } \\
\text { MAX } \\
\text { (F) }\end{array}$ & $\begin{array}{l}\text { TURE } \\
\text { MIN } \\
\text { (F) }\end{array}$ & $\begin{array}{l}\text { DRY } \\
\text { BULB } \\
(F)\end{array}$ \\
\hline $\begin{array}{l}127 \\
128 \\
129 \\
130 \\
131\end{array}$ & $\begin{array}{l}5 \\
5 \\
5 \\
5 \\
5\end{array}$ & $\begin{array}{r}7 \\
8 \\
9 \\
10 \\
11\end{array}$ & $\begin{array}{l}79 \\
79 \\
79 \\
79 \\
79\end{array}$ & $\begin{array}{l}50 . \\
49 . \\
39 . \\
35 . \\
32 .\end{array}$ & $\begin{array}{r}43 . \\
24 \\
23 \\
3 \\
5\end{array}$ & $\begin{array}{l}49 . \\
33{ }^{\circ} \\
28 . \\
20 \\
26 .\end{array}$ \\
\hline $\begin{array}{l}132 \\
133 \\
134 \\
135 \\
136\end{array}$ & $\begin{array}{l}5 \\
5 \\
5 \\
5 \\
5\end{array}$ & $\begin{array}{l}12 \\
13 \\
14 \\
15 \\
16\end{array}$ & $\begin{array}{l}79 \\
79 \\
79 \\
19 \\
79\end{array}$ & $\begin{array}{l}42 . \\
50 \\
58 . \\
62 . \\
64 .\end{array}$ & $\begin{array}{l}20 \\
26 \\
25{ }^{\circ} \\
24 \\
26^{\circ}\end{array}$ & $\begin{array}{l}25 \\
38 \\
46 \\
35 \\
40\end{array}$ \\
\hline $\begin{array}{l}137 \\
138 \\
139 \\
140 \\
141\end{array}$ & $\begin{array}{l}5 \\
5 \\
5 \\
5 \\
5\end{array}$ & $\begin{array}{l}17 \\
18 \\
19 \\
20 \\
21\end{array}$ & $\begin{array}{l}79 \\
79 \\
79 \\
79 \\
79\end{array}$ & $\begin{array}{l}61 . \\
62 . \\
64 . \\
62 . \\
46 .\end{array}$ & $\begin{array}{l}24 . \\
29 . \\
28 . \\
28 . \\
30 .\end{array}$ & $\begin{array}{l}45{ }^{\circ} \\
39{ }^{\circ} \\
44^{\circ} \\
40^{\circ} \\
41^{\circ}\end{array}$ \\
\hline $\begin{array}{l}142 \\
143 \\
144 \\
145 \\
146\end{array}$ & $\begin{array}{l}5 \\
5 \\
5 \\
5 \\
5\end{array}$ & $\begin{array}{l}22 \\
23 \\
24 \\
25 \\
26\end{array}$ & $\begin{array}{l}79 \\
79 \\
79 \\
79 \\
79\end{array}$ & $\begin{array}{l}59 . \\
65 . \\
66 . \\
65 . \\
62 .\end{array}$ & $\begin{array}{l}25 . \\
27{ }^{\circ} \\
37 . \\
29 \\
32 .\end{array}$ & $\begin{array}{l}51 . \\
40 . \\
48 . \\
46 . \\
46 .\end{array}$ \\
\hline $\begin{array}{l}147 \\
148 \\
149 \\
150 \\
151\end{array}$ & $\begin{array}{l}5 \\
5 \\
5 \\
5 \\
5\end{array}$ & $\begin{array}{l}27 \\
28 \\
29 \\
30 \\
31\end{array}$ & $\begin{array}{l}79 \\
79 \\
79 \\
79 \\
79\end{array}$ & $\begin{array}{l}65 . \\
66 . \\
65 . \\
66 . \\
42 .\end{array}$ & $\begin{array}{l}32 . \\
32 \\
29 \\
28 \\
25 .\end{array}$ & $\begin{array}{l}44^{\circ} \\
54{ }^{\circ} \\
45^{\circ} \\
33^{\circ} \\
30\end{array}$ \\
\hline
\end{tabular}


May-September 1977, 1978, and 1979--Continued

\begin{tabular}{|c|c|c|c|c|c|c|}
\hline $\begin{array}{l}\text { WET } \\
\text { BULB } \\
(F)\end{array}$ & $\begin{array}{l}\text { PAN } \\
\text { MAX } \\
\text { (F) }\end{array}$ & $\begin{array}{l}\text { WATER } \\
\text { MIN } \\
\text { (F) }\end{array}$ & $\begin{array}{l}\text { PRECIP } \\
(I N .)\end{array}$ & $\begin{array}{l}\text { WIND } \\
\text { (MI•/DAY) }\end{array}$ & $\begin{array}{l}\text { PAN } \\
\text { EVAP } \\
(I N \bullet)\end{array}$ & $\begin{array}{l}\text { SOLAR } \\
\text { RAD } \\
(L Y .)\end{array}$ \\
\hline $\begin{array}{l}43 . \\
33 . \\
28 . \\
18 \\
28\end{array}$ & $\begin{array}{l}* * 4 \\
* * 4 \\
* 4 * \\
* * 4 \\
* * 4\end{array}$ & 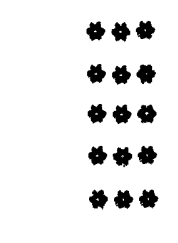 & $\begin{array}{l}0.00 \\
0.00 \\
0.00 \\
0.00 \\
0.00\end{array}$ & $\begin{array}{l}84 . \\
111 . \\
99 . \\
102 . \\
148 .\end{array}$ & $\begin{array}{l}* 40 \\
0 * 4 \\
* 40 \\
* 40\end{array}$ & $\begin{array}{l}14 \\
312 . \\
272 . \\
658 . \\
736 .\end{array}$ \\
\hline $\begin{array}{l}29 \\
34 \\
42 \\
34 \\
37\end{array}$ & $\begin{array}{l}* * * \\
* * 4 \\
* * * \\
* * 4 \\
* * *\end{array}$ & 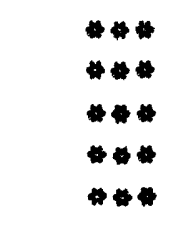 & $\begin{array}{l}.06 \\
0.00 \\
0.00 \\
0.00 \\
0.00\end{array}$ & $\begin{array}{l}118 \\
105 \\
113 \\
85 \\
78\end{array}$ & $\begin{array}{l}* * 4 \\
* * 4 \\
* * 4 \\
* * 4\end{array}$ & $\begin{array}{l}747 \\
766 . \\
685 . \\
658 . \\
541 .\end{array}$ \\
\hline $\begin{array}{l}40 . \\
37 \circ^{\circ} \\
39 . \\
38 . \\
39 .\end{array}$ & 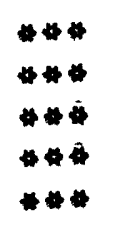 & 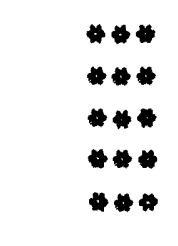 & $\begin{array}{r}0.00 \\
0.00 \\
0.00 \\
.01 \\
.07\end{array}$ & $\begin{array}{r}112 . \\
109 \\
79 \\
52 \\
88\end{array}$ & $\begin{array}{l}* * 4 \\
* * * \\
* * 4 \\
* * 4\end{array}$ & $\begin{array}{l}704 . \\
658^{\circ} \\
396^{\circ} \\
193^{\circ} \\
618^{\circ}\end{array}$ \\
\hline $\begin{array}{l}47 . \\
39{ }^{\circ} \\
45^{\circ} \\
44 \circ^{\circ} \\
43 .\end{array}$ & 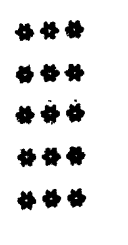 & 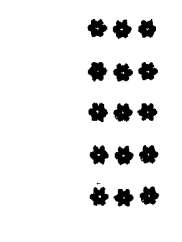 & $\begin{array}{r}0.00 \\
0.00 \\
0.00 \\
0.00 \\
.16\end{array}$ & $\begin{array}{l}97 . \\
76 . \\
74 . \\
52 . \\
51 .\end{array}$ & 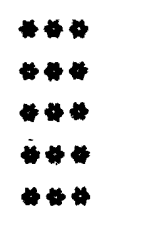 & $\begin{array}{l}734 . \\
688^{\circ} \\
534 . \\
363 . \\
706 .\end{array}$ \\
\hline $\begin{array}{l}43 . \\
49 . \\
44 \\
33 . \\
30\end{array}$ & 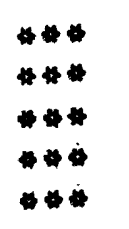 & 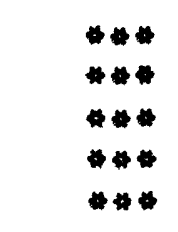 & $\begin{array}{r}0.00 \\
.02 \\
0.00 \\
.28 \\
.49\end{array}$ & $\begin{array}{r}81 . \\
115 . \\
105 . \\
46 . \\
79 .\end{array}$ & $\begin{array}{l}* 40 \\
* 40 \\
* 4 * \\
.40 \\
.22\end{array}$ & $\begin{array}{l}536 . \\
549 . \\
562 . \\
334 . \\
719 .\end{array}$ \\
\hline
\end{tabular}


Table 4.--Climatic data, Jefferson station,

\begin{tabular}{|c|c|c|c|c|c|c|}
\hline $\begin{array}{r}\text { DAY } \\
\text { OF } \\
\text { YEAR }\end{array}$ & MONTH & DAY & YEAR & $\begin{array}{l}\text { TEMF } \\
\text { MAX } \\
\text { (F) }\end{array}$ & $\begin{array}{l}\text { TURE } \\
\text { MIN } \\
\text { (F) }\end{array}$ & $\begin{array}{l}\text { DRY } \\
\text { BULB } \\
\text { (F) }\end{array}$ \\
\hline $\begin{array}{l}152 \\
153 \\
154 \\
155 \\
156\end{array}$ & $\begin{array}{l}6 \\
6 \\
6 \\
6 \\
6\end{array}$ & $\begin{array}{l}1 \\
2 \\
3 \\
4 \\
5\end{array}$ & $\begin{array}{l}79 \\
79 \\
79 \\
79 \\
79\end{array}$ & $\begin{array}{l}48 . \\
52 . \\
60 . \\
63 . \\
65 .\end{array}$ & $\begin{array}{l}24 . \\
29 . \\
28 . \\
30 . \\
32 .\end{array}$ & $\begin{array}{l}290^{\circ} \\
390^{\circ} \\
410^{\circ} \\
48 .\end{array}$ \\
\hline $\begin{array}{l}157 \\
158 \\
159 \\
160 \\
161\end{array}$ & $\begin{array}{l}6 \\
6 \\
6 \\
6 \\
6\end{array}$ & $\begin{array}{r}5 \\
7 \\
8 \\
9 \\
10\end{array}$ & $\begin{array}{l}79 \\
79 \\
79 \\
79 \\
79\end{array}$ & $\begin{array}{l}68 . \\
69 . \\
46 . \\
48 . \\
40 .\end{array}$ & $\begin{array}{l}34 . \\
33 . \\
30 . \\
27 . \\
27 .\end{array}$ & $\begin{array}{l}52 . \\
41 . \\
32 . \\
30 \\
31\end{array}$ \\
\hline $\begin{array}{l}162 \\
163 \\
164 \\
165 \\
166\end{array}$ & $\begin{array}{l}6 \\
6 \\
6 \\
6 \\
6\end{array}$ & $\begin{array}{l}11 \\
12 \\
13 \\
14 \\
15\end{array}$ & $\begin{array}{l}79 \\
79 \\
79 \\
79 \\
79\end{array}$ & $\begin{array}{l}59 . \\
65 . \\
71 . \\
72 . \\
69 .\end{array}$ & $\begin{array}{l}26 . \\
32 . \\
31 . \\
35 . \\
33 .\end{array}$ & $\begin{array}{l}49 . \\
49 . \\
50 \\
50 . \\
45 .\end{array}$ \\
\hline $\begin{array}{l}167 \\
168 \\
169 \\
170 \\
171\end{array}$ & $\begin{array}{l}6 \\
6 \\
6 \\
6 \\
6\end{array}$ & $\begin{array}{l}16 \\
17 \\
18 \\
19 \\
20\end{array}$ & $\begin{array}{l}79 \\
79 \\
79 \\
79 \\
79\end{array}$ & $\begin{array}{l}11 . \\
69 . \\
61 . \\
63 . \\
48 .\end{array}$ & $\begin{array}{l}28 . \\
280^{\circ} \\
270^{\circ} \\
30 . \\
28 .\end{array}$ & $\begin{array}{l}50 . \\
50 . \\
48 . \\
35 . \\
47 .\end{array}$ \\
\hline $\begin{array}{l}172 \\
173 \\
174 \\
175 \\
176\end{array}$ & $\begin{array}{l}6 \\
6 \\
6 \\
6 \\
6\end{array}$ & $\begin{array}{l}21 \\
22 \\
23 \\
24 \\
25\end{array}$ & $\begin{array}{l}79 \\
79 \\
79 \\
79 \\
79\end{array}$ & $\begin{array}{l}65 . \\
70 . \\
12 . \\
67 . \\
68 .\end{array}$ & $\begin{array}{l}27 . \\
30 . \\
31 . \\
430 \\
30\end{array}$ & $\begin{array}{l}40 . \\
50 \\
48 . \\
49 . \\
50 .\end{array}$ \\
\hline
\end{tabular}


May-September 1977, 1978, and 1979--Continued

\begin{tabular}{|c|c|c|c|c|c|c|}
\hline $\begin{array}{l}\text { WET } \\
\text { BULB } \\
(F)\end{array}$ & $\begin{array}{l}\text { PAN } \\
\text { MAX } \\
(F)\end{array}$ & $\begin{array}{l}\text { WATER } \\
\text { MIN } \\
\text { (F) }\end{array}$ & $\begin{array}{l}\text { PRECIP } \\
(I N .)\end{array}$ & $\begin{array}{l}\text { WIND } \\
\text { (MI./OAY) }\end{array}$ & $\begin{array}{l}\text { PAN } \\
\text { EVAP } \\
(I N .)\end{array}$ & $\begin{array}{l}\text { SOLAR } \\
\text { RAD } \\
(L Y .)\end{array}$ \\
\hline $\begin{array}{l}32 \\
35 \\
38 \\
42 \\
42\end{array}$ & $\begin{array}{l}72 . \\
72 . \\
72 . \\
72 . \\
70\end{array}$ & $\begin{array}{l}32 . \\
32 \\
39 \\
45 . \\
39 .\end{array}$ & $\begin{array}{r}0.00 \\
.05 \\
0.00 \\
0.00 \\
0.00\end{array}$ & $\begin{array}{l}48 . \\
60 \\
81 . \\
63 . \\
82 .\end{array}$ & $\begin{array}{l}.16 \\
.11 \\
.30 \\
.24 \\
.32\end{array}$ & $\begin{array}{l}582 \\
182 \\
193 . \\
621 . \\
654 .\end{array}$ \\
\hline $\begin{array}{l}43 . \\
37 . \\
33 . \\
32 . \\
33 .\end{array}$ & $\begin{array}{l}72 \\
75 \\
46 \\
10 \\
10\end{array}$ & $\begin{array}{l}39 \\
39 \\
36 \\
4 \\
4 \%\end{array}$ & $\begin{array}{l}0.00 \\
0.00 \\
2.85 \\
.98 \\
0.00\end{array}$ & $\begin{array}{r}120 \\
45 \\
25 \\
156 \\
38\end{array}$ & $\begin{array}{l}.36 \\
4 * 4 \\
* 4 * \\
* 44 \\
.28\end{array}$ & $\begin{array}{l}766^{\circ} \\
163 . \\
412^{\circ} \\
694 . \\
837 .\end{array}$ \\
\hline $\begin{array}{l}37 . \\
42 \\
42 \\
42 \\
41 .\end{array}$ & $\begin{array}{l}73 . \\
73 . \\
79 . \\
75 . \\
68 .\end{array}$ & $\begin{array}{l}36 . \\
37 . \\
41 . \\
41 . \\
41 .\end{array}$ & $\begin{array}{l}0.00 \\
0.00 \\
0.00 \\
0.00 \\
0.00\end{array}$ & $\begin{array}{l}69 . \\
62 . \\
78 . \\
96 . \\
89 .\end{array}$ & $\begin{array}{r}.26 \\
.31 \\
.36 \\
.29 \\
.33\end{array}$ & $\begin{array}{l}829 . \\
8150^{\circ} \\
821 . \\
609 \\
624^{\circ}\end{array}$ \\
\hline $\begin{array}{l}38 \\
41 . \\
35 \\
32 \\
37\end{array}$ & $\begin{array}{l}68 . \\
70 \\
70 \\
7 \% \\
68 .\end{array}$ & $\begin{array}{l}39 . \\
39 . \\
39 . \\
36 . \\
36 .\end{array}$ & $\begin{array}{r}0.00 \\
0.00 \\
0.00 \\
0.00 \\
.20\end{array}$ & $\begin{array}{r}82 . \\
98 . \\
159 . \\
194^{\circ} \\
85^{\circ}\end{array}$ & $\begin{array}{r}.32 \\
.37 \\
.29 \\
.16 \\
.33\end{array}$ & $\begin{array}{l}766 . \\
180^{\circ} \\
143^{\circ} \\
724 . \\
841^{\circ}\end{array}$ \\
\hline $\begin{array}{l}33 . \\
39 . \\
43 . \\
46 . \\
45 .\end{array}$ & $\begin{array}{l}73 \\
75 \\
75 \\
73 \\
72\end{array}$ & $\begin{array}{l}36 . \\
36 . \\
41 . \\
41 . \\
39 .\end{array}$ & $\begin{array}{l}0.00 \\
0.00 \\
0.00 \\
0.00 \\
0.00\end{array}$ & $\begin{array}{l}67 . \\
58 . \\
58 . \\
74 . \\
54 .\end{array}$ & $\begin{array}{l}.32 \\
.26 \\
.52 \\
.15 \\
.24\end{array}$ & $\begin{array}{l}847 . \\
670 . \\
628 . \\
495 . \\
648 .\end{array}$ \\
\hline
\end{tabular}


Table 4.--Climatic data, Jefferson station,

\begin{tabular}{|c|c|c|c|c|c|c|}
\hline $\begin{array}{r}\text { DAY } \\
\text { OF } \\
\text { YEAR }\end{array}$ & MONTH & DAY & YEAR & $\begin{array}{l}\text { IEMI } \\
\text { MAX } \\
\text { (F) }\end{array}$ & $\begin{array}{l}\text { URE } \\
\text { MIN } \\
\text { (F) }\end{array}$ & $\begin{array}{l}\text { DRY } \\
\text { BULB } \\
(\dot{F})\end{array}$ \\
\hline $\begin{array}{l}177 \\
178 \\
179 \\
180 \\
181\end{array}$ & $\begin{array}{l}6 \\
6 \\
6 \\
6 \\
6\end{array}$ & $\begin{array}{l}26 \\
27 \\
28 \\
29 \\
30\end{array}$ & $\begin{array}{l}19 \\
79 \\
79 \\
79 \\
79\end{array}$ & $\begin{array}{l}74 . \\
73{ }^{\circ} \\
75^{\circ} \\
750^{\circ} \\
16 .\end{array}$ & $\begin{array}{l}32 . \\
33{ }^{\circ} \\
34{ }^{\circ} \\
34 \\
36 .\end{array}$ & $\begin{array}{l}52 . \\
52 . \\
54 . \\
55 . \\
59 .\end{array}$ \\
\hline $\begin{array}{l}182 \\
183 \\
184 \\
185 \\
186\end{array}$ & $\begin{array}{l}7 \\
7 \\
7 \\
7 \\
7\end{array}$ & $\begin{array}{l}1 \\
2 \\
3 \\
4 \\
5\end{array}$ & $\begin{array}{l}79 \\
79 \\
79 \\
79 \\
19\end{array}$ & $\begin{array}{l}12 . \\
68 . \\
68 . \\
11 . \\
65 .\end{array}$ & $\begin{array}{l}41 . \\
31{ }^{\circ} \\
34 \\
32 . \\
34 .\end{array}$ & $\begin{array}{l}50 . \\
61 . \\
49 . \\
49 . \\
50 .\end{array}$ \\
\hline $\begin{array}{l}187 \\
188 \\
189 \\
190 \\
191\end{array}$ & $\begin{array}{l}7 \\
7 \\
7 \\
7 \\
7\end{array}$ & $\begin{array}{r}6 \\
7 \\
8 \\
9 \\
10\end{array}$ & $\begin{array}{l}79 \\
79 \\
79 \\
79 \\
79\end{array}$ & $\begin{array}{l}68 . \\
68 . \\
72 . \\
74 . \\
75 .\end{array}$ & $\begin{array}{l}34 . \\
31{ }^{\circ} \\
35{ }^{\circ} \\
35{ }^{\circ} \\
34\end{array}$ & $\begin{array}{l}52 . \\
47 . \\
54{ }^{\circ} \\
45 . \\
47^{\circ}\end{array}$ \\
\hline $\begin{array}{l}192 \\
193 \\
194 \\
195 \\
196\end{array}$ & $\begin{array}{l}7 \\
7 \\
7 \\
7 \\
7\end{array}$ & $\begin{array}{l}11 \\
12 \\
13 \\
14 \\
15\end{array}$ & $\begin{array}{l}79 \\
79 \\
79 \\
79 \\
79\end{array}$ & $\begin{array}{l}76 . \\
770^{\circ} \\
76 . \\
76 . \\
77 .\end{array}$ & $\begin{array}{l}33{ }^{\circ} \\
37 \circ^{\circ} \\
36 \\
37 \\
36 .\end{array}$ & $\begin{array}{l}57 \\
55 . \\
55 . \\
55 . \\
58\end{array}$ \\
\hline $\begin{array}{l}197 \\
198 \\
199 \\
200 \\
201\end{array}$ & $\begin{array}{l}7 \\
7 \\
7 \\
7 \\
7\end{array}$ & $\begin{array}{l}16 \\
17 \\
18 \\
19 \\
20\end{array}$ & $\begin{array}{l}79 \\
19 \\
79 \\
79 \\
79\end{array}$ & $\begin{array}{l}67 . \\
15 . \\
73 . \\
68 . \\
68 .\end{array}$ & $\begin{array}{l}37 . \\
32 . \\
32 . \\
28 . \\
29 .\end{array}$ & $\begin{array}{l}48 . \\
55^{\circ} \\
47^{\circ} \\
45^{\circ} \\
44^{\circ}\end{array}$ \\
\hline
\end{tabular}


May-September 1977, 1978, and 1979--Continued

WET

BULB

(F)

45.

48 .

48.

48.

47.

52.

44.

45.

45 .

47.

44.

47.

38.

43.

43.

47.

50.

50.

46.

48.

42.

41.

40.
PAN WATER

MAX

(F)

MIN

(F)

75.

68.

77.

77.

81.

73.

61.

70.

75.

70 .

77.

61.

77.

70 .

75.

77.

81.

73.

79.

77.

79.

73.

70.

61.

68.
39.

39.

41.

43.

45.

46.

41.

43.

41.

43.

43.

39.

43.

41.

43.

41.

45.

43.

43.

45.

0.00

0.00

.02

0.00

0.00

45.

41 .

41.

39.

39.
0.04

0.00

0.00

0.00
61.

68.

59.

43.

59.

62.

54.

44.

57.

40.

55.

76.

60.

40.

43.

66.

59.

47.

14.

48 .

59.

48.

43.
PAN SOLAR

EVAP RAD

(IN.) (LY.)

.21

.28

.32

.32

.17

553.

110.

763.

824.

435.

387.

533 .

556.

547.

.21652 .

23

392. 694.

716.

$\begin{array}{rl}.28 & 716 . \\ .41 & 740 . \\ .21 & 727\end{array}$

.21727.

.18

624.

487.

769.

727.

. 11 396.

$.29 \quad 528$.

.16448.

.17429.

.16556.

.20438. 
Table 4.--Climatic data, Jefferson station,

\begin{tabular}{|c|c|c|c|c|c|c|}
\hline $\begin{array}{r}\text { DAY } \\
\text { OF } \\
\text { YEAR }\end{array}$ & MONTH & DAY & YEAR & $\begin{array}{l}\text { TEMP } \\
\text { MAX } \\
\text { (F) }\end{array}$ & $\begin{array}{l}\text { TURE } \\
\text { MIN } \\
(F)\end{array}$ & $\begin{array}{l}\text { DRY } \\
\text { BULB } \\
(F)\end{array}$ \\
\hline $\begin{array}{l}202 \\
203 \\
204 \\
205 \\
206\end{array}$ & $\begin{array}{l}7 \\
7 \\
7 \\
7 \\
7\end{array}$ & $\begin{array}{l}21 \\
22 \\
23 \\
24 \\
25\end{array}$ & $\begin{array}{l}79 \\
79 \\
79 \\
79 \\
79\end{array}$ & $\begin{array}{l}70 . \\
70 . \\
71 . \\
70 . \\
70 .\end{array}$ & $\begin{array}{l}33{ }^{\circ} \\
43^{\circ} \\
32{ }^{\circ} \\
44{ }^{\circ} \\
41 .\end{array}$ & $\begin{array}{l}48 \\
58 \\
52 \\
55 . \\
57\end{array}$ \\
\hline $\begin{array}{l}207 \\
208 \\
209 \\
210 \\
211\end{array}$ & $\begin{array}{l}7 \\
7 \\
7 \\
7 \\
7\end{array}$ & $\begin{array}{l}26 \\
27 \\
28 \\
29 \\
30\end{array}$ & $\begin{array}{l}79 \\
79 \\
79 \\
79 \\
79\end{array}$ & $\begin{array}{l}69 . \\
73 . \\
74 . \\
16 . \\
73 .\end{array}$ & $\begin{array}{l}34 \\
35 \\
37 \\
36 \\
32\end{array}$ & $\begin{array}{l}56 . \\
54{ }^{\circ} \\
57^{\circ} \\
55^{\circ} \\
56 .\end{array}$ \\
\hline $\begin{array}{l}212 \\
213 \\
214 \\
215 \\
216\end{array}$ & $\begin{array}{l}7 \\
8 \\
8 \\
8 \\
8\end{array}$ & $\begin{array}{r}31 \\
1 \\
2 \\
3 \\
4\end{array}$ & $\begin{array}{l}79 \\
79 \\
79 \\
79 \\
79\end{array}$ & $\begin{array}{l}72 . \\
69 \\
72 \\
12 \\
74\end{array}$ & $\begin{array}{l}34 \\
32 \\
31 \\
34 \\
34\end{array}$ & $\begin{array}{l}42 . \\
47 . \\
49 . \\
52 . \\
50\end{array}$ \\
\hline $\begin{array}{l}217 \\
218 \\
219 \\
220 \\
221\end{array}$ & $\begin{array}{l}8 \\
8 \\
8 \\
8 \\
8\end{array}$ & $\begin{array}{l}5 \\
6 \\
7 \\
8 \\
9\end{array}$ & $\begin{array}{l}79 \\
79 \\
79 \\
79 \\
79\end{array}$ & $\begin{array}{l}80 . \\
79 . \\
80 . \\
19 . \\
75 .\end{array}$ & $\begin{array}{l}36 \\
34 \\
41 \\
45 \\
43\end{array}$ & $\begin{array}{l}55 . \\
57 . \\
60 . \\
52^{\circ} \\
58\end{array}$ \\
\hline $\begin{array}{l}222 \\
223 \\
224 \\
225 \\
226\end{array}$ & $\begin{array}{l}8 \\
8 \\
8 \\
8 \\
8\end{array}$ & $\begin{array}{l}10 \\
11 \\
12 \\
13 \\
14\end{array}$ & $\begin{array}{l}79 \\
79 \\
19 \\
79 \\
79\end{array}$ & $\begin{array}{l}69 . \\
63 . \\
11 . \\
13 . \\
69 .\end{array}$ & $\begin{array}{l}43 . \\
42{ }^{\circ} \\
36 \\
38 \\
43 .\end{array}$ & $\begin{array}{l}50 . \\
48 . \\
50 . \\
50 . \\
46 .\end{array}$ \\
\hline
\end{tabular}


May-September 1977, 1978, and 1979--Continued

$\begin{array}{lllllll}\text { WET } & \text { PAN WATER } & \text { PRECIP } & \text { WIND } & \text { PAN } & \text { SOLAR } \\ \text { BULB } & \text { MAX } & \text { MIN } & & & \text { EVAP } & \text { RAD } \\ \text { (F) } & \text { (F) } & \text { (F) } & \text { (IN.) } & \text { (MI./DAY) } & \text { (IN.) } & \text { (LY.) }\end{array}$

\begin{tabular}{|c|c|c|c|c|c|}
\hline $\begin{array}{l}45 . \\
49 \\
450^{\circ} \\
49 \\
52\end{array}$ & $\begin{array}{l}66 . \\
68 . \\
73 . \\
68 . \\
73 .\end{array}$ & $\begin{array}{l}39 \\
43 \\
41 \\
41 \\
48\end{array}$ & $\begin{array}{r}0.00 \\
.06 \\
0.00 \\
.01 \\
0.00\end{array}$ & $\begin{array}{l}52 . \\
60 \\
44 \\
45 . \\
55 .\end{array}$ & $\begin{array}{l}.14 \\
.24 \\
.15 \\
.23 \\
.30\end{array}$ \\
\hline
\end{tabular}

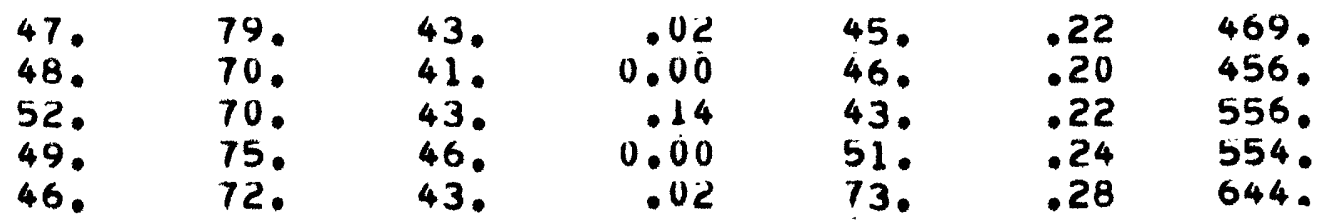

$\begin{array}{lllllll}40 . & 70 . & 43 . & 0.00 & 46 . & .28 & 485 . \\ 43 . & 70 . & 41 . & 0.00 & 69 . & .29 & 746 . \\ 41 . & 75 . & 41 . & 0.00 & 36 . & .22 & 379 . \\ 46 . & 72 . & 39 . & 0.00 & 45 . & .21 & 636 . \\ 40 . & 73 . & 39 . & 0.00 & 50 . & .29 & 113 .\end{array}$

\begin{tabular}{|c|c|c|c|c|c|}
\hline $\begin{array}{l}450^{\circ} \\
470^{\circ} \\
50^{\circ} \\
48 .\end{array}$ & $\begin{array}{l}79 . \\
79 . \\
75 . \\
82 . \\
75 .\end{array}$ & $\begin{array}{l}41 . \\
41 . \\
43 . \\
45 . \\
46 .\end{array}$ & $\begin{array}{r}0.00 \\
0.00 \\
0.00 \\
0.00 \\
.03\end{array}$ & $\begin{array}{l}41 . \\
70 \\
38, \\
41 . \\
28 .\end{array}$ & $\begin{array}{l}.28 \\
.28 \\
.32 \\
.15 \\
.27\end{array}$ \\
\hline
\end{tabular}

$\begin{array}{lllllll}49 . & 68 . & 48 . & .24 & 31 . & .4 & 261 . \\ 47 . & 61 . & 50 . & .08 & 41 . & .25 & 711 . \\ 45 . & 77 . & 48 . & 0.00 & 38 . & .19 & 431 . \\ 47 . & 73 . & 48 . & .04 & 42 . & .17 & 472 . \\ 45 . & 72 . & 48 . & .82 & 22 . & .29 & 163 .\end{array}$


Table 4.--Climatic data, Jefferson station,

\begin{tabular}{|c|c|c|c|c|c|c|}
\hline $\begin{array}{l}\text { DAY } \\
\text { OF } \\
\text { YEAR }\end{array}$ & MONTH & DAY & YEAR & $\begin{array}{l}\text { TEMP } \\
\text { MAX } \\
\text { (F) }\end{array}$ & $\begin{array}{l}\text { TURE } \\
\text { MIN } \\
\text { (F) }\end{array}$ & $\begin{array}{l}\text { DRY } \\
\text { BULB } \\
\text { (F) }\end{array}$ \\
\hline $\begin{array}{l}227 \\
228 \\
229 \\
230 \\
231\end{array}$ & $\begin{array}{l}8 \\
8 \\
8 \\
8 \\
8\end{array}$ & $\begin{array}{l}15 \\
16 \\
17 \\
18 \\
19\end{array}$ & $\begin{array}{l}79 \\
79 \\
79 \\
79 \\
79\end{array}$ & $\begin{array}{l}69 . \\
62 . \\
65 . \\
65 . \\
69 .\end{array}$ & $\begin{array}{l}42 . \\
38 \circ^{\circ} \\
320^{\circ} \\
33{ }^{\circ} \\
30\end{array}$ & $\begin{array}{l}470^{\circ} \\
45^{\circ} \\
44^{\circ}\end{array}$ \\
\hline $\begin{array}{l}232 \\
233 \\
234 \\
235 \\
236\end{array}$ & $\begin{array}{l}8 \\
8 \\
8 \\
8 \\
8\end{array}$ & $\begin{array}{l}20 \\
21 \\
22 \\
23 \\
24\end{array}$ & $\begin{array}{l}79 \\
79 \\
79 \\
79 \\
79\end{array}$ & $\begin{array}{l}61 . \\
59 . \\
59 . \\
61 . \\
66 .\end{array}$ & $\begin{array}{l}28 . \\
30 . \\
30 \\
30 . \\
33 .\end{array}$ & $\begin{array}{l}42 . \\
38 \\
45 \\
47 \\
42 .\end{array}$ \\
\hline $\begin{array}{l}237 \\
238 \\
239 \\
240 \\
241\end{array}$ & $\begin{array}{l}8 \\
8 \\
8 \\
8 \\
8\end{array}$ & $\begin{array}{l}25 \\
26 \\
27 \\
28 \\
29\end{array}$ & $\begin{array}{l}79 \\
79 \\
79 \\
79 \\
79\end{array}$ & $\begin{array}{l}66 . \\
64 . \\
64 . \\
69 \\
72 .\end{array}$ & $\begin{array}{l}39 . \\
32 . \\
28 . \\
31 . \\
29 .\end{array}$ & $\begin{array}{l}48 \\
44 \\
37 \\
38 \\
36\end{array}$ \\
\hline $\begin{array}{l}242 \\
243 \\
244 \\
245 \\
246\end{array}$ & $\begin{array}{l}8 \\
8 \\
9 \\
9 \\
9\end{array}$ & $\begin{array}{r}30 \\
31 \\
1 \\
2 \\
3\end{array}$ & $\begin{array}{l}79 \\
19 \\
79 \\
79 \\
79\end{array}$ & $\begin{array}{l}70 . \\
650^{\circ} \\
69 . \\
72 . \\
12 .\end{array}$ & $\begin{array}{l}32 . \\
30 . \\
27 . \\
29 . \\
29 .\end{array}$ & $\begin{array}{l}45 . \\
36 \\
44 \\
42 . \\
52\end{array}$ \\
\hline $\begin{array}{l}247 \\
248 \\
249 \\
250 \\
251\end{array}$ & $\begin{array}{l}9 \\
9 \\
9 \\
9 \\
9\end{array}$ & $\begin{array}{l}4 \\
5 \\
6 \\
7 \\
8\end{array}$ & $\begin{array}{l}79 \\
79 \\
79 \\
79 \\
79\end{array}$ & $\begin{array}{l}72 . \\
72 \\
75 \\
15 \\
75\end{array}$ & $\begin{array}{l}31 . \\
29 . \\
32 . \\
33 . \\
35 .\end{array}$ & $\begin{array}{l}49 . \\
480^{\circ} \\
350^{\circ} \\
44 . \\
42\end{array}$ \\
\hline
\end{tabular}


May-September 1977, 1978, and 1979--Continued

\begin{tabular}{|c|c|c|c|c|c|c|}
\hline $\begin{array}{l}\text { WET } \\
\text { BULB } \\
(F)\end{array}$ & $\begin{array}{l}\text { PAN } \\
\text { MAX } \\
\text { (F) }\end{array}$ & $\begin{array}{l}\text { WATER } \\
\text { MIN } \\
(F)\end{array}$ & $\begin{array}{l}\text { PRECIP } \\
\left(I N_{\bullet}\right)\end{array}$ & $\begin{array}{l}\text { WINO } \\
\text { (MI./DAY) }\end{array}$ & $\begin{array}{l}\text { PAN } \\
\text { EVAP } \\
(I N .)\end{array}$ & $\begin{array}{l}\text { SOLAR } \\
\text { RAD } \\
(L Y .)\end{array}$ \\
\hline $\begin{array}{l}45 . \\
45^{\circ} \\
34 \circ^{\circ} \\
42 . \\
39 .\end{array}$ & $\begin{array}{l}57 \\
18 \\
66 \\
68 \\
59\end{array}$ & $\begin{array}{l}46 . \\
46 . \\
43 . \\
41 \\
41 .\end{array}$ & $\begin{array}{r}.05 \\
.03 \\
0.00 \\
.84 \\
1.14\end{array}$ & $\begin{array}{l}43 . \\
29 . \\
33 . \\
47 . \\
39 .\end{array}$ & $\begin{array}{l}.11 \\
.13 \\
4 \$ 4 \\
.44 \\
.16\end{array}$ & $\begin{array}{l}367 \\
428 \\
456 \\
344 \\
497^{\circ}\end{array}$ \\
\hline $\begin{array}{l}38 . \\
36 . \\
40 \\
43 \\
37\end{array}$ & $\begin{array}{l}68 \\
61 \\
68 \\
68 \\
68\end{array}$ & $\begin{array}{l}39 . \\
41 . \\
39 . \\
41 . \\
41 .\end{array}$ & $\begin{array}{l}.03 \\
0.00 \\
0.00 \\
0.00 \\
0.00\end{array}$ & $\begin{array}{l}46 . \\
50 \\
45 . \\
40 \\
44 .\end{array}$ & $\begin{array}{l}.16 \\
.21 \\
.13 \\
.19 \\
.17\end{array}$ & $\begin{array}{l}390^{\circ} \\
569 . \\
566 . \\
495 . \\
567^{\circ}\end{array}$ \\
\hline $\begin{array}{l}44 \\
40 \\
34 \\
36 \\
32\end{array}$ & $\begin{array}{l}68 \\
72 \\
73 . \\
70 \\
72 .\end{array}$ & $\begin{array}{l}41 . \\
41 . \\
39 . \\
39 . \\
39 .\end{array}$ & $\begin{array}{l}0.00 \\
0.00 \\
0.00 \\
0.00 \\
0.00\end{array}$ & $\begin{array}{l}63 . \\
30 \\
48 \\
64 . \\
36 .\end{array}$ & $\begin{array}{l}.18 \\
.09 \\
.19 \\
.14 \\
.20\end{array}$ & $\begin{array}{l}481 . \\
390^{\circ} \\
575 . \\
550^{\circ} \\
622^{\circ}\end{array}$ \\
\hline $\begin{array}{l}41 . \\
34 . \\
37 . \\
36 . \\
42 .\end{array}$ & $\begin{array}{l}72 . \\
61 \\
70 \\
73 \\
68\end{array}$ & $\begin{array}{l}37 . \\
37 \circ^{\circ} \\
37 . \\
390^{\circ} \\
37 .\end{array}$ & $\begin{array}{l}0.00 \\
0.00 \\
0.00 \\
0.00 \\
0.00\end{array}$ & $\begin{array}{l}50 . \\
68 . \\
49 . \\
44 . \\
38 .\end{array}$ & $\begin{array}{l}.10 \\
.21 \\
.26 \\
.21 \\
.26\end{array}$ & $\begin{array}{l}392 . \\
611 . \\
649 . \\
444 . \\
598^{\circ} .\end{array}$ \\
\hline $\begin{array}{l}42 . \\
38 \\
33 . \\
39 . \\
39 .\end{array}$ & $\begin{array}{l}75 \\
75 \\
75 \\
75 \\
75\end{array}$ & $\begin{array}{l}39 . \\
41 . \\
37 . \\
37 . \\
39 .\end{array}$ & $\begin{array}{l}0.00 \\
0.00 \\
0.00 \\
0.00 \\
0.00\end{array}$ & $\begin{array}{l}38 . \\
37 \\
35 . \\
38 \\
30\end{array}$ & $\begin{array}{l}.12 \\
.21 \\
.10 \\
.22 \\
.15\end{array}$ & $\begin{array}{l}461 . \\
5670^{\circ} \\
540 . \\
517 . \\
399^{\circ}\end{array}$ \\
\hline
\end{tabular}


Table 4.--Climatic data, Jefferson station,

\begin{tabular}{|c|c|c|c|c|c|c|}
\hline $\begin{array}{l}\text { DAY } \\
\text { OF } \\
\text { YEAR }\end{array}$ & MONTH & DAY & YEAR & $\begin{array}{l}\text { TEMP } \\
\text { MAX } \\
\text { (F) }\end{array}$ & $\begin{array}{l}\text { TURE } \\
\text { MIN } \\
\text { (F) }\end{array}$ & $\begin{array}{l}\text { DRY } \\
\text { BULB } \\
\text { (F) }\end{array}$ \\
\hline $\begin{array}{l}252 \\
253 \\
254 \\
255 \\
256\end{array}$ & $\begin{array}{l}9 \\
9 \\
9 \\
9 \\
9\end{array}$ & $\begin{array}{r}9 \\
10 \\
11 \\
12 \\
13\end{array}$ & $\begin{array}{l}79 \\
79 \\
79 \\
79 \\
79\end{array}$ & $\begin{array}{l}75 . \\
74 . \\
72 . \\
11 . \\
64 .\end{array}$ & $\begin{array}{l}34 \\
34 \\
32 \\
32 \\
35\end{array}$ & $\begin{array}{l}40 . \\
42 . \\
46 . \\
39 \\
38 .\end{array}$ \\
\hline $\begin{array}{l}257 \\
258 \\
259 \\
260 \\
261\end{array}$ & $\begin{array}{l}9 \\
9 \\
9 \\
9 \\
9\end{array}$ & $\begin{array}{l}14 \\
15 \\
16 \\
17 \\
18\end{array}$ & $\begin{array}{l}79 \\
79 \\
79 \\
79 \\
79\end{array}$ & $\begin{array}{l}64 . \\
52 . \\
66 . \\
69 . \\
66 .\end{array}$ & $\begin{array}{l}310^{\circ} \\
10{ }^{\circ} \\
18{ }^{\circ} \\
26{ }^{\circ} \\
27\end{array}$ & $\begin{array}{l}33 . \\
24 . \\
44 . \\
49 \\
41\end{array}$ \\
\hline $\begin{array}{l}262 \\
263 \\
264 \\
265 \\
266\end{array}$ & $\begin{array}{l}9 \\
9 \\
9 \\
9 \\
9\end{array}$ & $\begin{array}{l}19 \\
20 \\
21 \\
22 \\
23\end{array}$ & $\begin{array}{l}79 \\
79 \\
79 \\
79 \\
79\end{array}$ & $\begin{array}{l}62 . \\
62 . \\
55 . \\
62 . \\
65 .\end{array}$ & $\begin{array}{l}24 \circ^{\circ} \\
28 \circ^{\circ} \\
33{ }^{\circ} \\
270^{\circ} \\
26\end{array}$ & $\begin{array}{l}30 \\
44 \\
38 \\
42 \\
38\end{array}$ \\
\hline $\begin{array}{l}267 \\
268 \\
269 \\
270 \\
271\end{array}$ & $\begin{array}{l}9 \\
9 \\
9 \\
9 \\
9\end{array}$ & $\begin{array}{l}24 \\
25 \\
26 \\
27 \\
28\end{array}$ & $\begin{array}{l}79 \\
79 \\
79 \\
79 \\
79\end{array}$ & $\begin{array}{l}68 . \\
68 . \\
650^{\circ} \\
64{ }^{\circ} \\
62 .\end{array}$ & $\begin{array}{l}30 . \\
28 . \\
29 . \\
28 . \\
23 .\end{array}$ & $\begin{array}{l}45 . \\
37 \\
33 \\
35 \\
33 .\end{array}$ \\
\hline 272 & 9 & 29 & 79 & 64. & 28. & \\
\hline
\end{tabular}


May-September 1977, 1978, and 1979--Continued

\begin{tabular}{|c|c|c|c|c|c|c|}
\hline $\begin{array}{l}\text { WET } \\
\text { BULB } \\
(F)\end{array}$ & $\begin{array}{l}\text { PAN } \\
\text { MAX } \\
\text { (F) }\end{array}$ & $\begin{array}{l}\text { WATER } \\
\text { MIN } \\
\text { (F) }\end{array}$ & $\begin{array}{l}\text { PRECIP } \\
(I N \cdot)\end{array}$ & $\begin{array}{l}\text { WIND } \\
\text { (MI./DAY) }\end{array}$ & $\begin{array}{l}\text { PAN } \\
\text { EVAP } \\
(I N \cdot)\end{array}$ & $\begin{array}{l}\text { SOLAK } \\
\text { RAD } \\
(L Y .)\end{array}$ \\
\hline $\begin{array}{l}37 . \\
38 \\
42 \\
32 \\
36\end{array}$ & $\begin{array}{l}70 \\
73 \\
70 \\
64 \\
68\end{array}$ & $\begin{array}{l}39 . \\
39 . \\
41 . \\
39 . \\
37 .\end{array}$ & $\begin{array}{r}0.00 \\
0.00 \\
0.00 \\
.09 \\
0.00\end{array}$ & $\begin{array}{l}31 . \\
53 . \\
47 . \\
49 . \\
51 .\end{array}$ & $\begin{array}{l}.18 \\
.27 \\
.11 \\
.21 \\
.15\end{array}$ & $\begin{array}{l}444 . \\
402^{\circ} \\
412^{\circ} \\
553 . \\
384 .\end{array}$ \\
\hline $\begin{array}{l}31 . \\
20 \\
34 . \\
37 \\
35 .\end{array}$ & $\begin{array}{l}64 . \\
17 \\
20 \\
68 \\
72^{\circ}\end{array}$ & $\begin{array}{l}36 . \\
32{ }^{\circ} \\
32 . \\
36 . \\
36 .\end{array}$ & $\begin{array}{l}0.00 \\
0.00 \\
0.00 \\
0.00 \\
0.00\end{array}$ & $\begin{array}{l}38 . \\
36 . \\
50 \\
73 . \\
47 .\end{array}$ & $\begin{array}{l}.07 \\
.19 \\
.27 \\
.23 \\
.19\end{array}$ & $\begin{array}{l}425 . \\
559 . \\
537 . \\
540 . \\
520 .\end{array}$ \\
\hline $\begin{array}{l}32 . \\
36 . \\
37 \\
37 \\
33 .\end{array}$ & $\begin{array}{l}70 . \\
59 . \\
50 . \\
66 . \\
63 .\end{array}$ & $\begin{array}{l}36 . \\
34{ }^{\circ} \\
37 \\
37 . \\
36 .\end{array}$ & $\begin{array}{r}0.00 \\
.22 \\
0.00 \\
0.00 \\
0.00\end{array}$ & $\begin{array}{l}66 . \\
44 . \\
35 \\
63 . \\
36 .\end{array}$ & $\begin{array}{l}.21 \\
.02 \\
.21 \\
.17 \\
.21\end{array}$ & $\begin{array}{l}409 . \\
190 . \\
462 . \\
429 . \\
380 .\end{array}$ \\
\hline $\begin{array}{l}37 \\
35 \\
32 \\
34 \\
32\end{array}$ & $\begin{array}{l}64 . \\
68 . \\
68 . \\
59 \\
61 .\end{array}$ & $\begin{array}{l}36 . \\
36{ }^{\circ} \\
34{ }^{\circ} \\
34 . \\
34{ }^{\circ}\end{array}$ & $\begin{array}{l}0.00 \\
0.00 \\
0.00 \\
0.00 \\
0.00\end{array}$ & $\begin{array}{l}47 . \\
39 . \\
61 . \\
54 . \\
70 .\end{array}$ & $\begin{array}{l}.16 \\
.05 \\
.24 \\
.16 \\
.18\end{array}$ & $\begin{array}{l}412 . \\
324 . \\
373{ }^{\circ} \\
379 . \\
454^{\circ}\end{array}$ \\
\hline 36. & 63. & 34. & 0.00 & 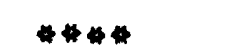 & 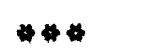 & $\$ 40$ \\
\hline
\end{tabular}



Table 5.--Climatic data, Fairplay station, May-September 1977, 1978 and 1979

TEMPERATURE MAX: Maximum daily air temperature

TEMPERATURE MIN: Minimum daily air temperature

PAN WATER MAX: Maximum daily water temperature of evaporation pan

PAN WATER MIN: Minimum daily water temperature of evaporation pan

PRECIP: Daily precipitation

PAN EVAP: Daily pan evaporation

DAILY AVG TEMP: Daily average air temperature

DAILY AVG REL HUM: Daily average relative humidity

SOLAR RAD: Daily average solar radiation

F: Degrees Fahrenheit

IN: Inches

MI : Mile

LY: Langleys per minute

$\star \star \star$ : Represents missing data 
Table 5.--Climatic data, Fairplay station,

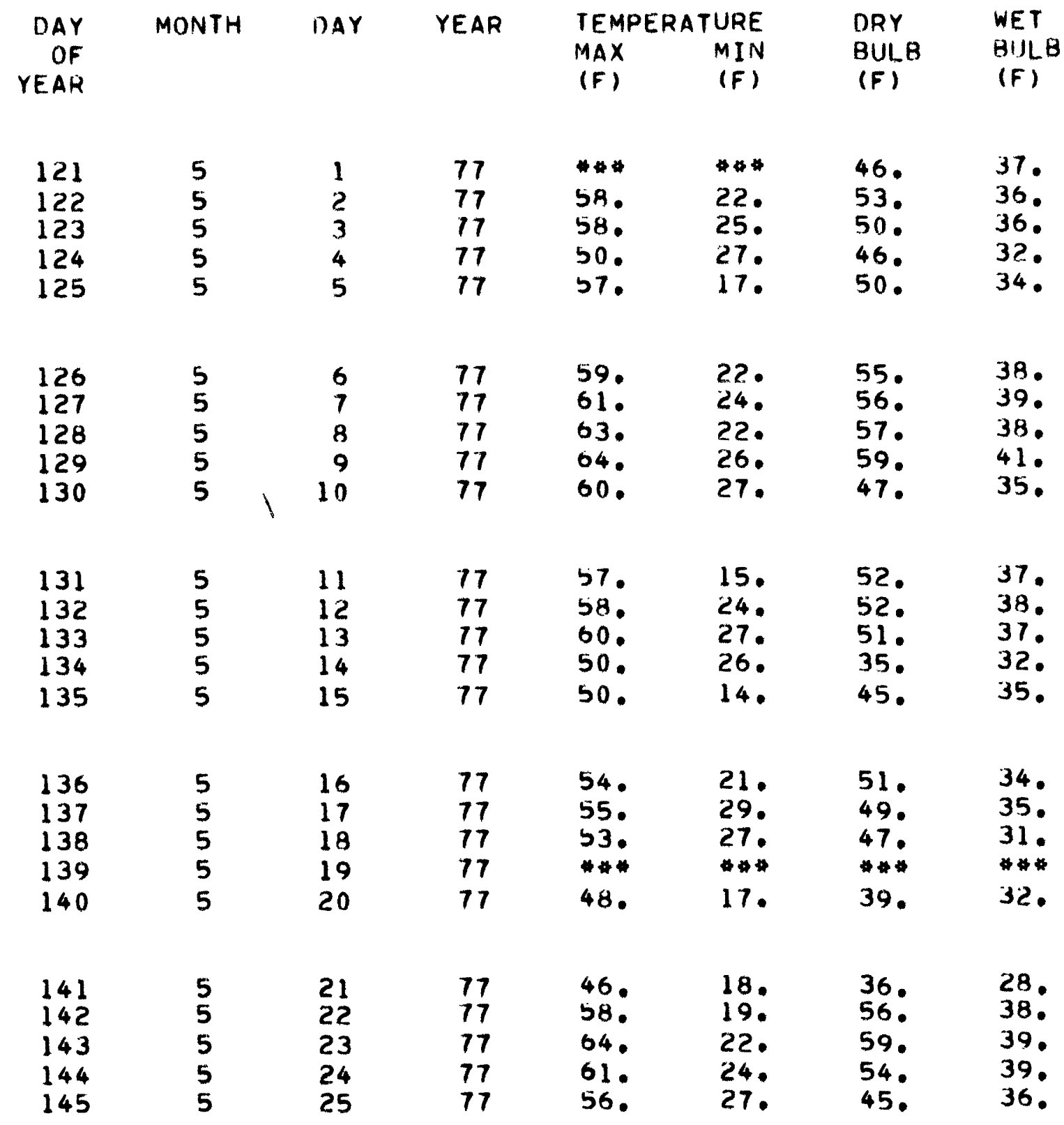


May-September 1977, 1978, and 1979--Continued

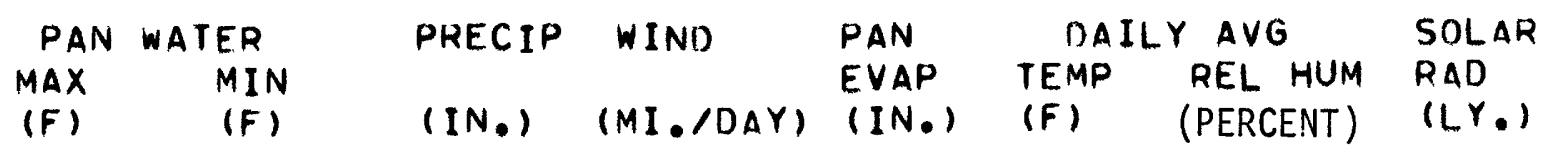

\begin{tabular}{|c|c|c|c|c|c|c|}
\hline $\begin{array}{l}4 \\
66 . \\
62 . \\
58 . \\
61 .\end{array}$ & $\begin{array}{l}36 . \\
36 . \\
36 . \\
34 .\end{array}$ & $\begin{array}{l}0.00 \\
0.00 \\
0.00 \\
0.00 \\
0.00\end{array}$ & $\begin{array}{r}4 \% \\
69 \\
115 . \\
165 \\
135\end{array}$ & $\begin{array}{l}.44 \\
.22 \\
.30 \\
.30 \\
.32\end{array}$ & 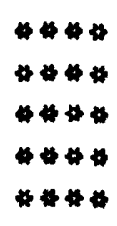 & 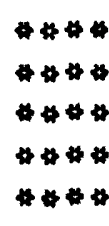 \\
\hline
\end{tabular}

\begin{tabular}{|c|c|c|c|c|c|c|}
\hline $\begin{array}{l}68 . \\
66 . \\
71 . \\
64 . \\
60\end{array}$ & $\begin{array}{l}35 . \\
35 . \\
36 . \\
38 \\
38\end{array}$ & $\begin{array}{l}0.00 \\
0.00 \\
0.00 \\
0.00 \\
0.00\end{array}$ & $\begin{array}{r}84 . \\
108 . \\
80 \\
90 \\
129\end{array}$ & $\begin{array}{l}.27 \\
.36 \\
.27 \\
.33 \\
.33\end{array}$ & 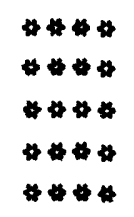 & 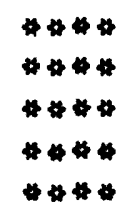 \\
\hline
\end{tabular}

\begin{tabular}{|c|c|c|c|c|c|c|}
\hline $\begin{array}{l}60 . \\
64 . \\
655^{\circ} \\
54 . \\
63 .\end{array}$ & $\begin{array}{l}32 . \\
37 \\
38 \\
38 \\
35\end{array}$ & $\begin{array}{r}0.00 \\
0.00 \\
0.00 \\
.26 \\
0.00\end{array}$ & $\begin{array}{r}117 . \\
86 . \\
90 \\
70 \\
160\end{array}$ & $\begin{array}{l}.30 \\
.26 \\
.24 \\
.06 \\
.22\end{array}$ & 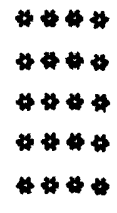 & 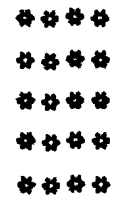 \\
\hline
\end{tabular}

\begin{tabular}{|c|c|c|c|c|c|c|c|}
\hline $\begin{array}{l}62 . \\
58 . \\
57 . \\
54 .\end{array}$ & $\begin{array}{l}38 . \\
35 \\
35 \\
33\end{array}$ & $\begin{array}{l}0.00 \\
0.00 \\
0.00 \\
0.00 \\
.04\end{array}$ & $\begin{array}{l}115 . \\
197 . \\
171 . \\
14 \\
184 .\end{array}$ & $\begin{array}{l}.32 \\
.37 \\
.35 \\
.44 \\
.32\end{array}$ & 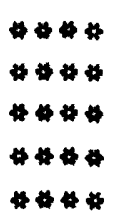 & 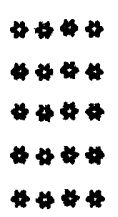 & $\begin{array}{l}619 \\
620^{\circ} \\
747 \\
541 \\
348\end{array}$ \\
\hline 54. & 33. & .04 & $184^{\circ}$ & .32 & 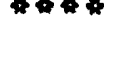 & & \\
\hline $\begin{array}{l}57 . \\
68 . \\
65 . \\
62 . \\
61 .\end{array}$ & $\begin{array}{l}31 . \\
330^{\circ} \\
37 . \\
38 \\
37 .\end{array}$ & $\begin{array}{l}0.00 \\
0.00 \\
0.00 \\
0.00 \\
0.00\end{array}$ & $\begin{array}{r}186 . \\
66 . \\
101 . \\
106 . \\
58 .\end{array}$ & $\begin{array}{r}.28 \\
.20 \\
.36 \\
.28 \\
.28\end{array}$ & 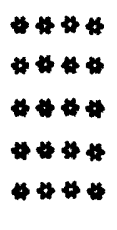 & 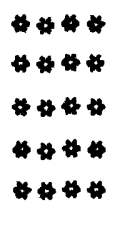 & $\begin{array}{l}785 \\
636 \\
693 \\
441 \\
554\end{array}$ \\
\hline
\end{tabular}


Table 5.--Climatic data, Fairplay station,

\begin{tabular}{|c|c|c|c|c|c|c|c|}
\hline $\begin{array}{r}\text { DAY } \\
\text { OF } \\
\text { YEAR }\end{array}$ & MONTH & DAY & YEAR & $\begin{array}{l}\text { TEMP } \\
\text { MAX } \\
\text { (F) }\end{array}$ & $\begin{array}{l}\text { URE } \\
\text { MIN } \\
\text { (F) }\end{array}$ & $\begin{array}{l}\text { DRY } \\
\text { BULB } \\
\text { (F) }\end{array}$ & $\begin{array}{l}\text { WET } \\
\text { RULB } \\
\text { (F) }\end{array}$ \\
\hline $\begin{array}{l}146 \\
147 \\
148 \\
149 \\
150\end{array}$ & $\begin{array}{l}5 \\
5 \\
5 \\
5 \\
5\end{array}$ & $\begin{array}{l}26 \\
27 \\
28 \\
29 \\
30\end{array}$ & $\begin{array}{l}77 \\
77 \\
77 \\
77 \\
77\end{array}$ & $\begin{array}{l}53 . \\
54 . \\
55 . \\
61 . \\
65 .\end{array}$ & $\begin{array}{l}20 . \\
20 . \\
21 . \\
27 . \\
27 .\end{array}$ & $\begin{array}{l}45 \\
50 \\
51 \\
61 \\
62\end{array}$ & $\begin{array}{l}35 \\
36 \\
36 \\
43 \\
43\end{array}$ \\
\hline $\begin{array}{l}151 \\
152 \\
153 \\
154 \\
155\end{array}$ & $\begin{array}{l}5 \\
6 \\
6 \\
6 \\
6\end{array}$ & $\begin{array}{r}31 \\
1 \\
2 \\
3 \\
4\end{array}$ & $\begin{array}{l}77 \\
77 \\
77 \\
77 \\
77\end{array}$ & $\begin{array}{l}70 . \\
72 . \\
69 . \\
67 . \\
71 .\end{array}$ & $\begin{array}{l}28 . \\
330^{\circ} \\
29 . \\
32 . \\
32 .\end{array}$ & $\begin{array}{l}64 \\
63 \\
55 \\
58 \\
63 \\
\end{array}$ & $\begin{array}{l}45 \\
45 \\
43 \\
44 \\
46\end{array}$ \\
\hline $\begin{array}{l}156 \\
157 \\
158 \\
159 \\
160\end{array}$ & $\begin{array}{l}6 \\
6 \\
6 \\
6 \\
6\end{array}$ & $\begin{array}{l}5 \\
6 \\
7 \\
8 \\
9\end{array}$ & $\begin{array}{l}77 \\
77 \\
77 \\
77 \\
77\end{array}$ & $\begin{array}{l}73 . \\
64 . \\
69 \\
67 . \\
65 .\end{array}$ & $\begin{array}{l}35 . \\
36 . \\
35 . \\
33 . \\
33 .\end{array}$ & $\begin{array}{l}64 . \\
52 . \\
56 . \\
53 . \\
59 .\end{array}$ & $\begin{array}{l}46 \\
46 \\
47 \\
46 \\
46\end{array}$ \\
\hline $\begin{array}{l}161 \\
162 \\
163 \\
164 \\
165\end{array}$ & $\begin{array}{l}6 \\
6 \\
6 \\
6 \\
6\end{array}$ & $\begin{array}{l}10 \\
11 \\
12 \\
13 \\
14\end{array}$ & $\begin{array}{l}77 \\
77 \\
77 \\
77 \\
77\end{array}$ & $\begin{array}{l}68 . \\
65 . \\
10 . \\
74 . \\
73 .\end{array}$ & $\begin{array}{l}29 . \\
35 . \\
31 . \\
35 . \\
39 .\end{array}$ & $\begin{array}{l}62 . \\
56 . \\
66 \\
61 \\
72 .\end{array}$ & $\begin{array}{l}43 . \\
450^{\circ} \\
440^{\circ} \\
50 \\
44 .\end{array}$ \\
\hline $\begin{array}{l}166 \\
167 \\
168 \\
169 \\
170\end{array}$ & $\begin{array}{l}6 \\
6 \\
6 \\
6 \\
6\end{array}$ & $\begin{array}{l}15 \\
16 \\
17 \\
18 \\
19\end{array}$ & $\begin{array}{l}77 \\
77 \\
77 \\
77 \\
77\end{array}$ & $\begin{array}{l}73^{\circ} \\
74^{\circ} \\
76^{\circ} \\
74^{\circ} \\
73^{\circ}\end{array}$ & $\begin{array}{l}36 . \\
29 . \\
39 . \\
39 . \\
31 .\end{array}$ & $\begin{array}{l}70 . \\
72 . \\
64 . \\
61 . \\
65 .\end{array}$ & $\begin{array}{l}45 . \\
45 . \\
50 \\
40 . \\
51\end{array}$ \\
\hline
\end{tabular}


May-September 1977, 1978, and 1979--Continued

\begin{tabular}{|c|c|c|c|c|c|c|}
\hline $\begin{array}{l}\text { PAN } \\
\text { MAX } \\
\text { (F) }\end{array}$ & $\begin{array}{l}\text { WATER } \\
\text { MIN } \\
\text { (F) }\end{array}$ & $\begin{array}{l}\text { PRECIP } \\
(I N \cdot)\end{array}$ & $\begin{array}{l}\text { WIND } \\
\text { (MI./DAY) }\end{array}$ & $\begin{array}{l}\text { PAN } \\
\text { EVAP } \\
\text { (IN.) }\end{array}$ & $\begin{array}{l}\text { DAIL } \\
\text { TEMP } \\
\text { (F) }\end{array}$ & $\begin{array}{l}\text { Y AVG } \\
\text { REL HUM } \\
\text { (PERCENT) }\end{array}$ \\
\hline
\end{tabular}

\begin{tabular}{|c|c|c|c|c|c|c|}
\hline $\begin{array}{l}57 . \\
63 . \\
62 . \\
69 .\end{array}$ & $\begin{array}{l}35 \\
35 \\
35 \\
42 \\
37\end{array}$ & $\begin{array}{l}0.00 \\
0.00 \\
0.00 \\
0.00 \\
0.00\end{array}$ & $\begin{array}{r}157 . \\
88 . \\
92 \\
79 \\
88 .\end{array}$ & $\begin{array}{l}.20 \\
.21 \\
.22 \\
.24 \\
.29\end{array}$ & 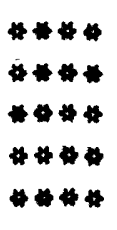 & 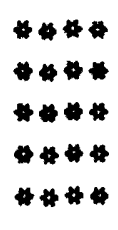 \\
\hline
\end{tabular}

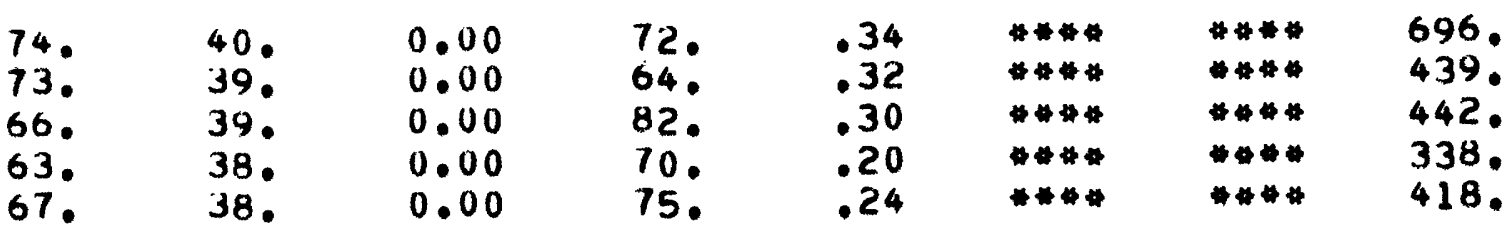

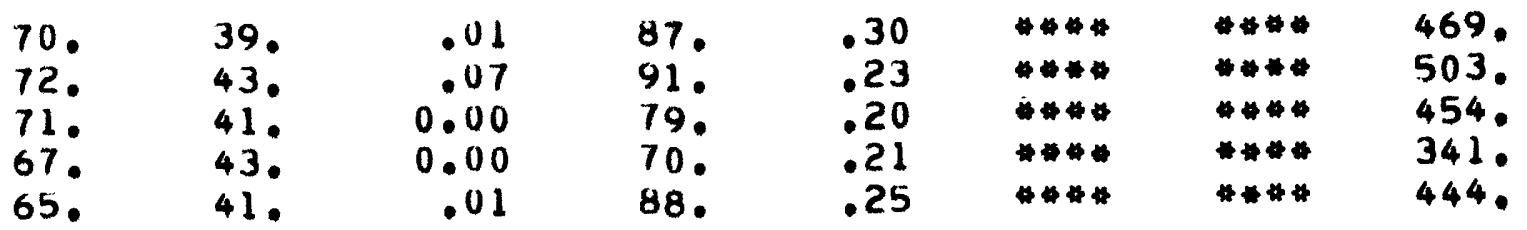

\begin{tabular}{|c|c|c|c|c|c|c|}
\hline $\begin{array}{l}73{ }^{\circ} \\
69^{\circ} \\
74^{\circ} \\
74^{\circ} \\
74^{\circ}\end{array}$ & $\begin{array}{l}38 . \\
39 . \\
38 . \\
41 . \\
47 .\end{array}$ & $\begin{array}{r}0.00 \\
0.00 \\
.05 \\
0.00 \\
.07\end{array}$ & $\begin{array}{r}101 . \\
90 . \\
76 . \\
79 . \\
92 .\end{array}$ & $\begin{array}{l}.34 \\
.30 \\
.26 \\
.40 \\
.41\end{array}$ & 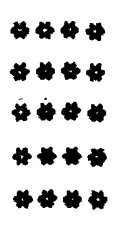 & $\begin{array}{l}* * * * \\
* * * * \\
* * * * \\
* * * * \\
* * * *\end{array}$ \\
\hline $\begin{array}{l}75 . \\
770^{\circ} \\
77 . \\
74 . \\
70\end{array}$ & $\begin{array}{l}38 . \\
38 . \\
38 . \\
49 \\
39\end{array}$ & $\begin{array}{l}0.00 \\
0.00 \\
0.00 \\
0.00 \\
0.00\end{array}$ & $\begin{array}{r}104 . \\
84 . \\
116 . \\
118 . \\
81 .\end{array}$ & $\begin{array}{l}.48 \\
.45 \\
.46 \\
.51 \\
.34\end{array}$ & 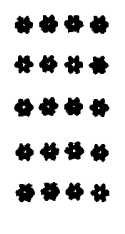 & 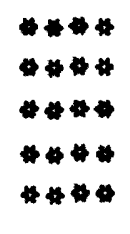 \\
\hline
\end{tabular}


Table 5.--Climatic data, Fairplay station,

\begin{tabular}{|c|c|c|c|c|c|c|c|}
\hline $\begin{array}{r}\text { DAY } \\
\text { OF } \\
\text { YEAR }\end{array}$ & MONTH & DAY & YEAR & $\begin{array}{l}\text { TEMP } \\
\text { MAX } \\
\text { (F) }\end{array}$ & $\begin{array}{l}\text { URE } \\
\text { MIN } \\
\text { (F) }\end{array}$ & $\begin{array}{l}\text { ORY } \\
\text { BiJLB } \\
\text { (F) }\end{array}$ & $\begin{array}{l}\text { WET } \\
\text { BULB } \\
\text { (F) }\end{array}$ \\
\hline $\begin{array}{l}171 \\
172 \\
173 \\
174 \\
175\end{array}$ & $\begin{array}{l}6 \\
6 \\
6 \\
6 \\
6\end{array}$ & $\begin{array}{l}20 \\
21 \\
22 \\
23 \\
24\end{array}$ & $\begin{array}{l}77 \\
77 \\
77 \\
77 \\
77\end{array}$ & $\begin{array}{l}68 . \\
66 . \\
69 . \\
650^{\circ} \\
63 .\end{array}$ & $\begin{array}{l}36 . \\
31 . \\
30 \\
38 \\
36 .\end{array}$ & $\begin{array}{l}64 . \\
64{ }^{\circ} \\
62{ }^{\circ} \\
51 . \\
57^{\circ}\end{array}$ & $\begin{array}{l}46 . \\
44 \circ^{\circ} \\
45^{\circ} \\
44^{\circ} \\
47^{\circ}\end{array}$ \\
\hline $\begin{array}{l}176 \\
177 \\
178 \\
179 \\
180\end{array}$ & $\begin{array}{l}6 \\
6 \\
6 \\
6 \\
6\end{array}$ & $\begin{array}{l}25 \\
26 \\
27 \\
28 \\
29\end{array}$ & $\begin{array}{l}17 \\
77 \\
77 \\
77 \\
77\end{array}$ & $\begin{array}{l}10 . \\
140^{\circ} \\
15 \circ \\
16 . \\
13 .\end{array}$ & $\begin{array}{l}33 . \\
41 . \\
40 . \\
42 . \\
36 .\end{array}$ & $\begin{array}{l}56 . \\
630^{\circ} \\
68 . \\
68 . \\
71 .\end{array}$ & $\begin{array}{l}46 . \\
48 \\
48 \\
55 \\
47\end{array}$ \\
\hline $\begin{array}{l}181 \\
182 \\
183 \\
184 \\
185\end{array}$ & $\begin{array}{l}6 \\
7 \\
7 \\
7 \\
7\end{array}$ & $\begin{array}{r}30 \\
1 \\
2 \\
3 \\
4\end{array}$ & $\begin{array}{l}77 \\
77 \\
77 \\
77 \\
77\end{array}$ & $\begin{array}{l}74 . \\
73 \\
75 \\
75 \\
71 .\end{array}$ & $\begin{array}{l}37 . \\
32{ }^{\circ} \\
430^{\circ} \\
43 . \\
41 .\end{array}$ & $\begin{array}{l}72 . \\
69 . \\
71 . \\
66 . \\
62 .\end{array}$ & $\begin{array}{l}47 \\
48 \\
50 \\
49 \\
50\end{array}$ \\
\hline $\begin{array}{l}186 \\
187 \\
188 \\
189 \\
190\end{array}$ & $\begin{array}{l}7 \\
7 \\
7 \\
7 \\
7\end{array}$ & $\begin{array}{l}5 \\
6 \\
7 \\
8 \\
9\end{array}$ & $\begin{array}{l}77 \\
77 \\
77 \\
77 \\
77\end{array}$ & $\begin{array}{l}67 . \\
66 . \\
71 . \\
67 . \\
67 .\end{array}$ & $\begin{array}{l}40 . \\
35 . \\
36 . \\
34 . \\
32 .\end{array}$ & $\begin{array}{l}58 . \\
59 \\
62 \\
51 \\
60\end{array}$ & $\begin{array}{l}48 \\
47 \\
47 \\
44 \\
48\end{array}$ \\
\hline $\begin{array}{l}191 \\
192 \\
193 \\
194 \\
195\end{array}$ & $\begin{array}{l}7 \\
7 \\
7 \\
7 \\
7\end{array}$ & $\begin{array}{l}10 \\
11 \\
12 \\
13 \\
14\end{array}$ & $\begin{array}{l}77 \\
77 \\
77 \\
77 \\
77\end{array}$ & $\begin{array}{l}13 . \\
12 . \\
14 . \\
67 . \\
10\end{array}$ & $\begin{array}{l}33 . \\
34 \circ^{\circ} \\
350^{\circ} \\
43 . \\
37\end{array}$ & $\begin{array}{l}68 \\
70 \\
66 \\
65 \\
60\end{array}$ & $\begin{array}{l}43 . \\
47^{\circ} \\
470^{\circ} \\
50 \\
49 .\end{array}$ \\
\hline
\end{tabular}


May-September 1977, 1978, and 1979--Continued

\begin{tabular}{|c|c|c|c|c|c|c|c|}
\hline $\begin{array}{l}\text { PAN } \\
\text { MAX } \\
(F)\end{array}$ & $\begin{array}{l}\text { WATER } \\
\text { MIN } \\
\text { (F) }\end{array}$ & $\begin{array}{l}\text { PRECIP } \\
(\text { IN.) }\end{array}$ & $\begin{array}{l}\text { WIND } \\
\text { (MI./DAY) }\end{array}$ & $\begin{array}{l}\text { PAN } \\
\text { EVAP } \\
(I N \cdot)\end{array}$ & $\begin{array}{l}\text { DA } \\
\text { TEMP } \\
(F)\end{array}$ & $\begin{array}{l}\text { Y AVG } \\
\text { REL HUM } \\
\text { (PERCENT) }\end{array}$ & $\begin{array}{l}\text { SOLAR } \\
\text { RAD } \\
\text { (LY.) }\end{array}$ \\
\hline $\begin{array}{l}68 \\
66 \\
71 \\
65 \\
65\end{array}$ & $\begin{array}{l}44 \\
38 \\
39 \\
43 \\
42^{\circ}\end{array}$ & $\begin{array}{r}0.00 \\
0.00 \\
0.00 \\
0.00 \\
.04\end{array}$ & $\begin{array}{l}110 . \\
99 . \\
64 . \\
60 \\
62 .\end{array}$ & $\begin{array}{l}.40 \\
.31 \\
.31 \\
.22 \\
.17\end{array}$ & $\begin{array}{c}440 \\
50 \\
53 \\
49 \\
49\end{array}$ & $\begin{array}{l}4 \% \\
26 \\
45 \\
78 \\
76\end{array}$ & $\begin{array}{l}580^{\circ} \\
567^{\circ} \\
516^{\circ} \\
344^{\circ} \\
465^{\circ}\end{array}$ \\
\hline $\begin{array}{l}73 . \\
77 \\
77 \\
78 \\
75\end{array}$ & $\begin{array}{l}39 \\
45 \\
42 \\
45 \\
48\end{array}$ & $\begin{array}{l}0.00 \\
0.00 \\
0.00 \\
0.00 \\
0.00\end{array}$ & $\begin{array}{r}13 . \\
136 . \\
102 . \\
90 . \\
101 .\end{array}$ & $\begin{array}{l}.27 \\
.41 \\
.38 \\
.44 \\
.41\end{array}$ & $\begin{array}{l}54 . \\
59 . \\
59 . \\
59 . \\
58 .\end{array}$ & $\begin{array}{l}56 . \\
48 . \\
51 . \\
57 \\
45^{\circ}\end{array}$ & $\begin{array}{l}619 . \\
636 . \\
611^{\circ} \\
707^{\circ} \\
667 .\end{array}$ \\
\hline $\begin{array}{l}77 . \\
73 \\
75 \\
73 \\
72\end{array}$ & $\begin{array}{l}40 \\
40 \\
46 . \\
44 \\
48^{\circ}\end{array}$ & $\begin{array}{l}0.00 \\
0.00 \\
0.00 \\
0.00 \\
.02\end{array}$ & $\begin{array}{r}98 . \\
95 . \\
122 \\
87 \\
68\end{array}$ & $\begin{array}{l}.46 \\
.40 \\
.48 \\
.39 \\
.26\end{array}$ & $\begin{array}{l}55 . \\
57 . \\
62 . \\
59 . \\
56 .\end{array}$ & $\begin{array}{l}38 . \\
45 . \\
37 . \\
50 . \\
82 .\end{array}$ & $\begin{array}{l}749 . \\
543^{\circ} \\
706 . \\
393^{\circ} \\
426 .\end{array}$ \\
\hline $\begin{array}{l}68 \\
65 \\
71 . \\
73 \\
68\end{array}$ & $\begin{array}{l}45 . \\
41 \\
40 \\
43 \\
39\end{array}$ & $\begin{array}{r}0.00 \\
0.00 \\
0.00 \\
0.00 \\
.04\end{array}$ & $\begin{array}{l}85 . \\
89 . \\
69 . \\
87 . \\
68 .\end{array}$ & $\begin{array}{l}.24 \\
.29 \\
.27 \\
.36 \\
.17\end{array}$ & $\begin{array}{l}55 . \\
51 . \\
54 . \\
52 . \\
50 .\end{array}$ & $\begin{array}{l}72 . \\
69 . \\
57 . \\
74 . \\
71 .\end{array}$ & $\begin{array}{l}431 . \\
458 \\
487 \\
516 . \\
508\end{array}$ \\
\hline $\begin{array}{l}73 . \\
70 \\
74 . \\
67 . \\
68 .\end{array}$ & $\begin{array}{l}38 \\
37 \\
40 \\
46 \\
43\end{array}$ & $\begin{array}{l}0.00 \\
0.00 \\
0.00 \\
.01 \\
0.00\end{array}$ & $\begin{array}{c}103 \\
108 \\
84 \\
69 \\
82\end{array}$ & $\begin{array}{l}.40 \\
.42 \\
.41 \\
.26 \\
.26\end{array}$ & $\begin{array}{l}56 . \\
54 . \\
60 \\
55 . \\
55 .\end{array}$ & $\begin{array}{l}38 . \\
29 . \\
37 . \\
66 . \\
66 .\end{array}$ & $\begin{array}{l}762 . \\
582 . \\
503 . \\
384 . \\
431 .\end{array}$ \\
\hline
\end{tabular}


Table 5.--Climatic data, Fairplay station,

\begin{tabular}{|c|c|c|c|c|c|c|c|}
\hline $\begin{array}{r}\text { DAY } \\
\text { OF } \\
\text { YEAR }\end{array}$ & MONTH & DAY & YEAR & $\begin{array}{l}\text { TEMF } \\
\text { MAX } \\
\text { (F) }\end{array}$ & $\begin{array}{l}\text { FRE } \\
\text { MIN } \\
\text { (F) }\end{array}$ & $\begin{array}{l}\text { DRY } \\
\text { BULB } \\
\text { (F) }\end{array}$ & $\begin{array}{l}\text { WET } \\
\text { BULB } \\
\text { (F) }\end{array}$ \\
\hline $\begin{array}{l}196 \\
197 \\
198 \\
199 \\
200\end{array}$ & $\begin{array}{l}7 \\
7 \\
7 \\
7 \\
7\end{array}$ & $\begin{array}{l}15 \\
16 \\
17 \\
18 \\
19\end{array}$ & $\begin{array}{l}77 \\
77 \\
77 \\
77 \\
77\end{array}$ & $\begin{array}{l}73 . \\
77 . \\
75 . \\
78 . \\
75 .\end{array}$ & $\begin{array}{l}36 . \\
34 . \\
41 . \\
40 . \\
45 .\end{array}$ & $\begin{array}{l}61 . \\
71 \\
66 \\
64 \\
60\end{array}$ & $\begin{array}{l}50 . \\
50 . \\
51: \\
50 . \\
54 .\end{array}$ \\
\hline $\begin{array}{l}201 \\
202 \\
203 \\
204 \\
205\end{array}$ & $\begin{array}{l}7 \\
7 \\
7 \\
7 \\
7\end{array}$ & $\begin{array}{l}20 \\
21 \\
22 \\
23 \\
24\end{array}$ & $\begin{array}{l}77 \\
77 \\
77 \\
77 \\
77\end{array}$ & $\begin{array}{l}67 . \\
61 . \\
65 . \\
68 . \\
64 .\end{array}$ & $\begin{array}{l}37 . \\
400^{\circ} \\
460^{\circ} \\
420^{\circ} \\
33 .\end{array}$ & $\begin{array}{l}55 . \\
55 . \\
57 \\
60 \\
55\end{array}$ & $\begin{array}{l}52 . \\
52 . \\
53 . \\
53 . \\
51 .\end{array}$ \\
\hline $\begin{array}{l}206 \\
207 \\
208 \\
209 \\
210\end{array}$ & $\begin{array}{l}7 \\
7 \\
7 \\
7 \\
7\end{array}$ & $\begin{array}{l}25 \\
26 \\
27 \\
28 \\
29\end{array}$ & $\begin{array}{l}77 \\
77 \\
77 \\
77 \\
77\end{array}$ & $\begin{array}{l}61 . \\
70 . \\
68 . \\
67 . \\
73 .\end{array}$ & $\begin{array}{l}44^{\circ} \\
44^{\circ} \\
39^{\circ} \\
38^{\circ} \\
38^{\circ}\end{array}$ & $\begin{array}{l}56 . \\
59 \\
64: \\
67 \\
64\end{array}$ & $\begin{array}{l}53 . \\
550^{\circ} \\
51 . \\
50 . \\
47 .\end{array}$ \\
\hline $\begin{array}{l}211 \\
212 \\
213 \\
214 \\
215\end{array}$ & $\begin{array}{l}7 \\
7 \\
8 \\
8 \\
8\end{array}$ & $\begin{array}{r}30 \\
31 \\
1 \\
2 \\
3\end{array}$ & $\begin{array}{l}77 \\
77 \\
77 \\
77 \\
77\end{array}$ & $\begin{array}{l}12 . \\
11 . \\
10 . \\
72 . \\
75 .\end{array}$ & $\begin{array}{l}38 . \\
37 . \\
38 . \\
35 . \\
34 .\end{array}$ & $\begin{array}{l}69 . \\
49 . \\
68 \\
69 \\
72 .\end{array}$ & $\begin{array}{l}47 \\
46 \\
47 \\
47 \\
48\end{array}$ \\
\hline $\begin{array}{l}216 \\
217 \\
218 \\
219 \\
220\end{array}$ & $\begin{array}{l}8 \\
8 \\
8 \\
8 \\
8\end{array}$ & $\begin{array}{l}4 \\
5 \\
6 \\
7 \\
8\end{array}$ & $\begin{array}{l}77 \\
77 \\
77 \\
77 \\
77\end{array}$ & $\begin{array}{l}69 . \\
66 . \\
73 . \\
77 . \\
71 .\end{array}$ & $\begin{array}{l}35 . \\
42 . \\
40 \\
37 \\
35 .\end{array}$ & $\begin{array}{l}52 . \\
60 . \\
69 . \\
68 . \\
61 .\end{array}$ & $\begin{array}{l}49 . \\
49 . \\
50 . \\
49 . \\
51 .\end{array}$ \\
\hline
\end{tabular}


May-September 1977, 1978, and 1979--Continued

\begin{tabular}{|c|c|c|c|c|c|c|}
\hline$a x$ & $\begin{array}{l}\text { ATER } \\
\text { MIN } \\
\text { (F) }\end{array}$ & 11 & $\begin{array}{l}W I \\
\text { (MI }\end{array}$ & $\begin{array}{l}\text { PAN } \\
\text { EVAP } \\
(I N,)\end{array}$ & $\begin{array}{l}\text { DAILY AVG } \\
\text { TEMP } \\
\text { REL HUM } \\
\text { (F) } \\
\text { (PERCENT) }\end{array}$ & \\
\hline
\end{tabular}

\begin{tabular}{|c|c|c|c|c|c|c|c|}
\hline $\begin{array}{l}711^{\circ} \\
74 \circ^{\circ} \\
72 \circ^{\circ} \\
77 \circ^{\circ} \\
76 .\end{array}$ & $\begin{array}{l}43 . \\
42 . \\
45^{\circ} \\
45^{\circ} \\
49 .\end{array}$ & $\begin{array}{r}.11 \\
0.00 \\
0.00 \\
0.00 \\
.10\end{array}$ & $\begin{array}{l}66 . \\
54 . \\
88 . \\
68 . \\
80 .\end{array}$ & $\begin{array}{r}.26 \\
.22 \\
.36 \\
.33 \\
.32\end{array}$ & $\begin{array}{l}54 . \\
60 . \\
60 . \\
50 . \\
59 .\end{array}$ & $\begin{array}{l}71 . \\
49 . \\
55 . \\
59 . \\
76 .\end{array}$ & $\begin{array}{l}415 . \\
451 . \\
431 . \\
429 . \\
439 .\end{array}$ \\
\hline $\begin{array}{l}68 . \\
61 . \\
68 . \\
76 . \\
68 .\end{array}$ & $\begin{array}{l}46 . \\
50 \\
49 . \\
49 . \\
46 .\end{array}$ & $\begin{array}{r}.09 \\
1.70 \\
.38 \\
.03 \\
.52\end{array}$ & $\begin{array}{l}52 . \\
35 . \\
46 . \\
50 . \\
56 .\end{array}$ & $\begin{array}{l}.16 \\
.42 \\
.44 \\
.18 \\
.38\end{array}$ & $\begin{array}{l}53 . \\
51 . \\
54 . \\
55 . \\
52 .\end{array}$ & $\begin{array}{l}87 . \\
96 . \\
91 . \\
78 . \\
85 .\end{array}$ & $\begin{array}{l}304 \\
264 \\
382 \\
518 \\
340\end{array}$ \\
\hline
\end{tabular}

\begin{tabular}{|c|c|c|c|c|c|c|}
\hline $\begin{array}{l}64 . \\
75 . \\
70 \\
70 \\
74 .\end{array}$ & $\begin{array}{l}49 . \\
480^{\circ} \\
46{ }^{\circ} \\
44{ }^{\circ} \\
44 .\end{array}$ & $\begin{array}{r}.04 \\
.42 \\
.08 \\
.07 \\
0.00\end{array}$ & $\begin{array}{l}47 . \\
41 . \\
67 . \\
66 . \\
74 .\end{array}$ & $\begin{array}{l}.18 \\
.05 \\
.29 \\
.21 \\
.35\end{array}$ & $\begin{array}{l}50 . \\
54 . \\
53 . \\
54 . \\
56 .\end{array}$ & $\begin{array}{l}87 . \\
79 . \\
71 . \\
61 . \\
53 .\end{array}$ \\
\hline $\begin{array}{l}72 . \\
74 . \\
75 \\
75 . \\
72 .\end{array}$ & $\begin{array}{l}45^{\circ} \\
38 \circ^{\circ} \\
440^{\circ} \\
40 . \\
41 .\end{array}$ & $\begin{array}{l}.01 \\
0.00 \\
0.00 \\
0.00 \\
0.00\end{array}$ & $\begin{array}{r}112 . \\
106 \\
65 . \\
78 . \\
82 .\end{array}$ & $\begin{array}{r}.38 \\
.46 \\
.28 \\
.37 \\
.35\end{array}$ & $\begin{array}{l}57 . \\
56 . \\
54 . \\
56 . \\
58 .\end{array}$ & $\begin{array}{l}45^{\circ} \\
33 . \\
56 . \\
35^{\circ} \\
45^{\circ}\end{array}$ \\
\hline $\begin{array}{l}72 . \\
65 . \\
76 \\
76 . \\
74 .\end{array}$ & $\begin{array}{l}43 \\
45^{\circ} \\
45^{\circ} \\
43 . \\
46 .\end{array}$ & $\begin{array}{r}.07 \\
.09 \\
0.00 \\
0.00 \\
.01\end{array}$ & $\begin{array}{l}74 . \\
76 . \\
75 . \\
73 . \\
91 .\end{array}$ & $\begin{array}{r}.26 \\
.20 \\
.28 \\
.35 \\
.31\end{array}$ & $\begin{array}{l}53 . \\
52 . \\
58 . \\
59 . \\
57 .\end{array}$ & $\begin{array}{l}75 . \\
77 . \\
55 . \\
49 . \\
65 .\end{array}$ \\
\hline
\end{tabular}


Table 5.--Climatic data, Faimplay station,

\begin{tabular}{|c|c|c|c|c|c|c|c|}
\hline $\begin{array}{r}\text { DAY } \\
\text { OF } \\
\text { YEAR }\end{array}$ & MONTH & DAY & YEAR & $\begin{array}{l}\text { TEMF } \\
\text { MAX } \\
\text { (F) }\end{array}$ & $\begin{array}{l}\text { TURE } \\
\text { MIN } \\
\text { (F) }\end{array}$ & $\begin{array}{l}\text { DRY } \\
\text { BULB } \\
\text { (F) }\end{array}$ & $\begin{array}{l}\text { WET } \\
\text { BULB } \\
\text { (F) }\end{array}$ \\
\hline $\begin{array}{l}221 \\
222 \\
223 \\
224 \\
225\end{array}$ & $\begin{array}{l}8 \\
8 \\
8 \\
8 \\
8\end{array}$ & $\begin{array}{l}9 \\
10 \\
11 \\
12 \\
13\end{array}$ & $\begin{array}{l}77 \\
77 \\
77 \\
77 \\
77\end{array}$ & $\begin{array}{l}73 . \\
11 . \\
68 . \\
71 . \\
68 .\end{array}$ & $\begin{array}{l}36 . \\
39 \\
35 \circ \\
37 \\
36\end{array}$ & $\begin{array}{l}72 . \\
61 . \\
54 . \\
660^{\circ} \\
64 .\end{array}$ & $\begin{array}{l}51 . \\
50 . \\
46 . \\
49 . \\
48 .\end{array}$ \\
\hline $\begin{array}{l}226 \\
227 \\
228 \\
229 \\
230\end{array}$ & $\begin{array}{l}8 \\
8 \\
8 \\
8 \\
8\end{array}$ & $\begin{array}{l}14 \\
15 \\
16 \\
17 \\
18\end{array}$ & $\begin{array}{l}77 \\
77 \\
77 \\
17 \\
77\end{array}$ & $\begin{array}{l}70 . \\
64{ }^{\circ} \\
64 . \\
63 . \\
65^{\circ}\end{array}$ & $\begin{array}{l}34{ }^{\circ} \\
410^{\circ} \\
44{ }^{\circ} \\
43 \\
46\end{array}$ & $\begin{array}{l}60 . \\
59 . \\
59 . \\
56 . \\
60\end{array}$ & $\begin{array}{l}51 . \\
53 . \\
54 . \\
53 . \\
51 .\end{array}$ \\
\hline $\begin{array}{l}231 \\
232 \\
233 \\
234 \\
235\end{array}$ & $\begin{array}{l}8 \\
8 \\
8 \\
8 \\
8\end{array}$ & $\begin{array}{l}19 \\
20 \\
21 \\
22 \\
23\end{array}$ & $\begin{array}{l}77 \\
77 \\
77 \\
77 \\
77\end{array}$ & $\begin{array}{l}68 . \\
68 . \\
62 . \\
68 . \\
70\end{array}$ & $\begin{array}{l}41 . \\
390^{\circ} \\
380^{\circ} \\
37 . \\
40 .\end{array}$ & $\begin{array}{l}56 . \\
58 . \\
57 \\
65 \\
66 .\end{array}$ & $\begin{array}{l}52 . \\
49 . \\
49 . \\
49 . \\
50 .\end{array}$ \\
\hline $\begin{array}{l}236 \\
237 \\
238 \\
239 \\
240\end{array}$ & $\begin{array}{l}8 \\
8 \\
8 \\
8 \\
8\end{array}$ & $\begin{array}{l}24 \\
25 \\
26 \\
27 \\
28\end{array}$ & $\begin{array}{l}77 \\
77 \\
77 \\
77 \\
77\end{array}$ & $\begin{array}{l}70 . \\
69 . \\
72 . \\
69 . \\
60 .\end{array}$ & $\begin{array}{l}38 . \\
36 . \\
34 . \\
40 \\
30\end{array}$ & $\begin{array}{l}58 \\
61 \\
70 \\
47 \\
57\end{array}$ & $\begin{array}{l}48 . \\
47 . \\
50 \\
36 . \\
42 .\end{array}$ \\
\hline $\begin{array}{l}241 \\
242 \\
243 \\
244 \\
245\end{array}$ & $\begin{array}{l}8 \\
8 \\
8 \\
9 \\
9\end{array}$ & $\begin{array}{r}29 \\
30 \\
31 \\
1 \\
2\end{array}$ & $\begin{array}{l}77 \\
77 \\
17 \\
77 \\
77\end{array}$ & $\begin{array}{l}69 . \\
68 . \\
68 . \\
10 . \\
11 .\end{array}$ & $\begin{array}{l}28 . \\
35 . \\
32 . \\
31 . \\
37 .\end{array}$ & $\begin{array}{l}63 . \\
60 \\
57 \\
66 . \\
60\end{array}$ & $\begin{array}{l}45^{\circ} \\
46{ }^{\circ} \\
44{ }^{\circ} \\
48^{\circ}\end{array}$ \\
\hline
\end{tabular}


May-September 1977, 1978, and 1979--Continued

\begin{tabular}{|c|c|c|c|c|c|c|c|}
\hline $\begin{array}{l}\text { PAN } \\
\text { MAX } \\
\text { (F) }\end{array}$ & $\begin{array}{r}\text { WATER } \\
\text { MIN } \\
\text { (F) }\end{array}$ & $\begin{array}{l}\text { PRECIP } \\
(I N .)\end{array}$ & $\begin{array}{l}\text { WIND } \\
\text { (MI./DAY) }\end{array}$ & $\begin{array}{l}\text { PAN } \\
\text { EVAP } \\
\text { (IN.) }\end{array}$ & $\begin{array}{l}\text { DA } \\
\text { TEMP } \\
\text { (F) }\end{array}$ & $\begin{array}{l}\text { Y AVG } \\
\text { REL HUM } \\
\text { (PERCENT) }\end{array}$ & $\begin{array}{l}\text { SOLAR } \\
\text { RAD } \\
(L Y,)\end{array}$ \\
\hline $\begin{array}{l}71 . \\
70 . \\
72 . \\
72 . \\
69 .\end{array}$ & $\begin{array}{l}43 . \\
51 . \\
48 . \\
43 . \\
41 .\end{array}$ & $\begin{array}{l}0.00 \\
.01 \\
0.00 \\
0.00 \\
0.00\end{array}$ & $\begin{array}{l}61 . \\
51 . \\
53 . \\
54 . \\
57 .\end{array}$ & $\begin{array}{l}.25 \\
.25 \\
.22 \\
.20 \\
.23\end{array}$ & $\begin{array}{l}55 . \\
55 . \\
52 . \\
55 . \\
53 .\end{array}$ & $\begin{array}{l}61 . \\
78 . \\
75 . \\
50 . \\
58 .\end{array}$ & $\begin{array}{l}3930^{\circ} \\
382^{\circ} \\
4350^{\circ} \\
474^{\circ}\end{array}$ \\
\hline $\begin{array}{l}69 . \\
64 . \\
67 . \\
69 . \\
68 .\end{array}$ & $\begin{array}{l}41 . \\
490^{\circ} \\
500^{\circ} \\
50^{\circ} \\
53 .\end{array}$ & $\begin{array}{l}.05 \\
.06 \\
.09 \\
.04 \\
.02\end{array}$ & $\begin{array}{l}64 . \\
38 . \\
33 . \\
50 . \\
55 .\end{array}$ & $\begin{array}{l}.22 \\
.09 \\
.10 \\
.14 \\
.11\end{array}$ & $\begin{array}{l}54 . \\
\text { b2: } \\
53 . \\
53 . \\
55 .\end{array}$ & $\begin{array}{l}69 . \\
81 . \\
94 . \\
90 . \\
79 .\end{array}$ & $\begin{array}{l}425 . \\
245 . \\
295^{\circ} \\
305^{\circ} \\
338 .\end{array}$ \\
\hline $\begin{array}{l}70 . \\
68 . \\
64 . \\
72 . \\
73 .\end{array}$ & $\begin{array}{l}450^{\circ} \\
480^{\circ} \\
44{ }^{\circ} \\
45^{\circ} \\
45^{\circ}\end{array}$ & $\begin{array}{r}.01 \\
.05 \\
.03 \\
.01 \\
0.00\end{array}$ & $\begin{array}{l}61 . \\
58 . \\
52 . \\
79 . \\
63 .\end{array}$ & $\begin{array}{l}.14 \\
.19 \\
.15 \\
.22 \\
.26\end{array}$ & $\begin{array}{l}54 . \\
53 . \\
50 . \\
53 . \\
56 .\end{array}$ & $\begin{array}{l}80 . \\
74{ }^{\circ} \\
81^{\circ} \\
67^{\circ} \\
59^{\circ}\end{array}$ & $\begin{array}{l}478 . \\
2950^{\circ} \\
379 \\
5030^{\circ} \\
438\end{array}$ \\
\hline $\begin{array}{l}70 . \\
70 . \\
72 . \\
69 . \\
69 .\end{array}$ & $\begin{array}{l}45 . \\
44{ }^{\circ} \\
410^{\circ} \\
440^{\circ} \\
38 .\end{array}$ & $\begin{array}{l}0.00 \\
0.00 \\
0.00 \\
0.00 \\
0.00\end{array}$ & $\begin{array}{r}70 \\
76 \\
89 . \\
107 \\
78 .\end{array}$ & $\begin{array}{l}.27 \\
.25 \\
.30 \\
.32 \\
.26\end{array}$ & $\begin{array}{l}54 . \\
53 . \\
58 . \\
48 . \\
44 .\end{array}$ & $\begin{array}{l}67 . \\
67 . \\
56 . \\
52 . \\
53 .\end{array}$ & $\begin{array}{l}379 \\
431 . \\
618 \\
595 \\
540\end{array}$ \\
\hline $\begin{array}{l}72 . \\
68 . \\
63 . \\
71 . \\
69\end{array}$ & $\begin{array}{l}38 . \\
380^{\circ} \\
39 . \\
390^{\circ} \\
41 .\end{array}$ & $\begin{array}{l}0.00 \\
0.00 \\
0.00 \\
0.00 \\
0.00\end{array}$ & $\begin{array}{l}85 . \\
110 . \\
71 . \\
90 . \\
53 .\end{array}$ & $\begin{array}{r}.32 \\
.31 \\
.24 \\
.27 \\
.25\end{array}$ & $\begin{array}{l}49 . \\
52 . \\
50 . \\
52 . \\
53 .\end{array}$ & $\begin{array}{l}43 . \\
46 . \\
58 . \\
48 . \\
55^{\circ}\end{array}$ & $\begin{array}{l}634 . \\
459^{\circ} \\
350 . \\
549^{\circ} \\
361^{\circ}\end{array}$ \\
\hline
\end{tabular}


Table 5.--Climatic data, Fairplay station,

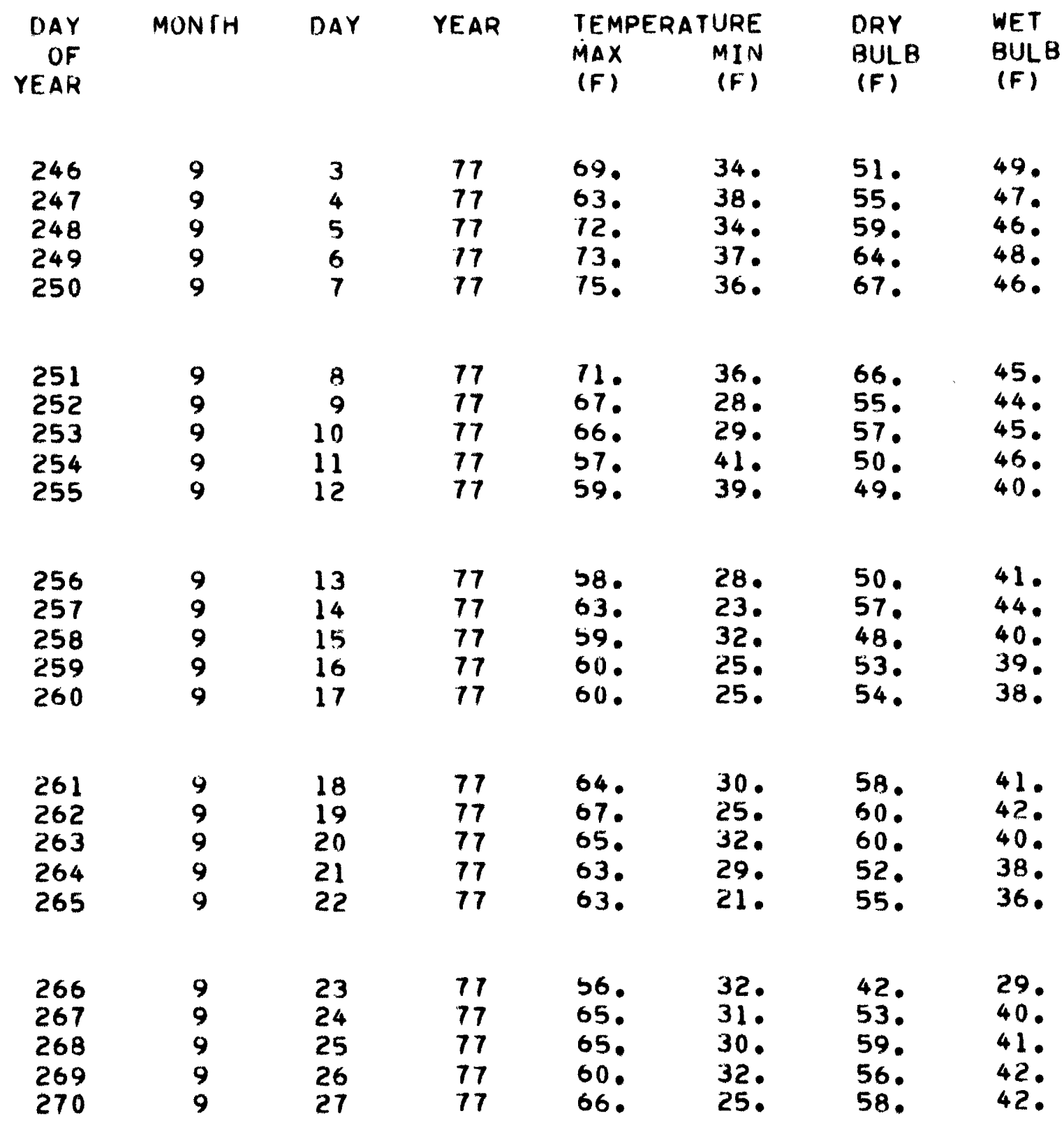


May-September 1977, 1978, and 1979--Continued

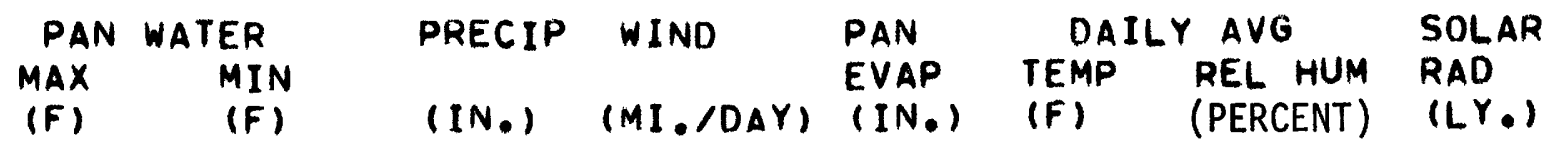

\begin{tabular}{|c|c|c|c|c|c|c|}
\hline $\begin{array}{l}65 . \\
66 . \\
72 . \\
72 . \\
73\end{array}$ & $\begin{array}{l}42 . \\
41 . \\
40 \\
39 \\
42 .\end{array}$ & $\begin{array}{l}0.00 \\
0.00 \\
0.00 \\
0.00 \\
0.00\end{array}$ & $\begin{array}{l}49 . \\
69 . \\
43 . \\
64 . \\
58 .\end{array}$ & $\begin{array}{l}.18 \\
.18 \\
.19 \\
.23 \\
.25\end{array}$ & $\begin{array}{l}50 . \\
\text { 50. } \\
45 . \\
53 . \\
54 .\end{array}$ & $\begin{array}{l}72 . \\
68 . \\
67 . \\
54 . \\
54 .\end{array}$ \\
\hline
\end{tabular}

\begin{tabular}{|c|c|c|c|c|c|c|c|}
\hline $\begin{array}{l}70 . \\
66 . \\
63 . \\
60 \\
64 .\end{array}$ & $\begin{array}{l}41 . \\
390^{\circ} \\
380^{\circ} \\
44 \circ^{\circ} \\
43 .\end{array}$ & $\begin{array}{r}0.00 \\
0.00 \\
0.00 \\
.08 \\
.01\end{array}$ & $\begin{array}{r}105 . \\
89 \\
72 \\
40 \\
94\end{array}$ & $\begin{array}{l}.38 \\
.30 \\
.21 \\
.06 \\
.20\end{array}$ & $\begin{array}{l}53 . \\
48, \\
48, \\
45, \\
39 .\end{array}$ & $\begin{array}{l}49 . \\
55 . \\
58 . \\
95 . \\
68 .\end{array}$ & $\begin{array}{l}504 . \\
458 . \\
278 . \\
194 . \\
456 .\end{array}$ \\
\hline $\begin{array}{l}61 . \\
63 . \\
61 . \\
57 . \\
62 .\end{array}$ & $\begin{array}{l}38 . \\
36 . \\
39 . \\
37^{\circ} \\
37^{\circ}\end{array}$ & $\begin{array}{l}0.00 \\
0.00 \\
0.00 \\
0.00 \\
0.00\end{array}$ & $\begin{array}{r}35 . \\
49 . \\
83 . \\
90 . \\
127 .\end{array}$ & $\begin{array}{l}.16 \\
.17 \\
.23 \\
.20 \\
.31\end{array}$ & $\begin{array}{l}40 . \\
42 . \\
45 . \\
43 . \\
44 .\end{array}$ & $\begin{array}{l}71 . \\
57 . \\
61 . \\
55 \\
38\end{array}$ & $\begin{array}{l}387 . \\
412 . \\
410 \\
363 . \\
369 .\end{array}$ \\
\hline $\begin{array}{l}65 . \\
64 . \\
66 . \\
65 . \\
60 .\end{array}$ & $\begin{array}{l}37 . \\
37 . \\
39 . \\
41 . \\
37 .\end{array}$ & $\begin{array}{l}0.00 \\
0.00 \\
0.00 \\
0.00 \\
0.00\end{array}$ & $\begin{array}{r}92 . \\
73 . \\
107 . \\
87 \\
101 .\end{array}$ & $\begin{array}{l}.29 \\
.28 \\
.32 \\
.27 \\
.31\end{array}$ & $\begin{array}{l}46 . \\
40 . \\
50 . \\
440^{\circ} \\
43 .\end{array}$ & $\begin{array}{l}24 . \\
48 . \\
39 . \\
42 . \\
51 .\end{array}$ & $\begin{array}{l}527 . \\
478 . \\
490 \\
458 . \\
471 .\end{array}$ \\
\hline $\begin{array}{l}55 . \\
63 . \\
66 . \\
63 . \\
65\end{array}$ & $\begin{array}{l}35 . \\
36 \\
38 \\
37 \\
37\end{array}$ & $\begin{array}{l}0.00 \\
0.00 \\
0.00 \\
0.00 \\
0.00\end{array}$ & $\begin{array}{r}188 . \\
106 . \\
120 \\
90^{\circ} \\
70\end{array}$ & $\begin{array}{l}.28 \\
.26 \\
.32 \\
.28 \\
.24\end{array}$ & $\begin{array}{l}39 . \\
490^{\circ} \\
49 . \\
44 . \\
46 .\end{array}$ & $\begin{array}{l}46 . \\
20 . \\
31 . \\
34 . \\
49 .\end{array}$ & $\begin{array}{l}544 . \\
4650^{\circ} \\
480 \\
5640^{\circ} \\
399^{\circ}\end{array}$ \\
\hline
\end{tabular}


Table 5.--Climatic data, Fairplay station,

\begin{tabular}{|c|c|c|c|c|c|c|c|}
\hline $\begin{array}{r}\text { DAY } \\
\text { OF } \\
\text { YEAR }\end{array}$ & MONTH & DAY & YEAR & $\begin{array}{l}\text { TEMF } \\
\text { MAX } \\
\text { (F) }\end{array}$ & $\begin{array}{l}\text { URE } \\
\text { MIN } \\
\text { (F) }\end{array}$ & $\begin{array}{l}\text { DRY } \\
\text { BULB } \\
\text { (F) }\end{array}$ & $\begin{array}{l}\text { WET } \\
\text { BULB } \\
(F)\end{array}$ \\
\hline $\begin{array}{l}271 \\
272 \\
273 \\
274 \\
121\end{array}$ & $\begin{array}{r}9 \\
9 \\
9 \\
10 \\
5\end{array}$ & $\begin{array}{r}28 \\
29 \\
30 \\
1 \\
1\end{array}$ & $\begin{array}{l}77 \\
77 \\
77 \\
77 \\
78\end{array}$ & $\begin{array}{l}67^{\circ} \\
67^{\circ} \\
590^{\circ} \\
54^{\circ} \\
45^{\circ}\end{array}$ & $\begin{array}{l}30 . \\
30 . \\
36 . \\
20 . \\
22 .\end{array}$ & $\begin{array}{l}60 . \\
58 . \\
40 . \\
42 . \\
28 .\end{array}$ & $\begin{array}{l}43 \\
39 \\
33 \\
40 \\
26\end{array}$ \\
\hline $\begin{array}{l}122 \\
123 \\
124 \\
125 \\
126\end{array}$ & $\begin{array}{l}5 \\
5 \\
5 \\
5 \\
5\end{array}$ & $\begin{array}{l}2 \\
3 \\
4 \\
5 \\
6\end{array}$ & $\begin{array}{l}78 \\
78 \\
78 \\
78 \\
78\end{array}$ & $\begin{array}{l}38 . \\
45 . \\
43 \\
35 \\
33\end{array}$ & $\begin{array}{l}20 . \\
22 . \\
20 . \\
11 . \\
19 .\end{array}$ & $\begin{array}{l}39 . \\
410^{\circ} \\
260^{\circ} \\
33 \circ \\
28 .\end{array}$ & $\begin{array}{l}34 . \\
31 . \\
26 . \\
32 . \\
28 .\end{array}$ \\
\hline $\begin{array}{l}127 \\
128 \\
129 \\
130 \\
131\end{array}$ & $\begin{array}{l}5 \\
5 \\
5 \\
5 \\
5\end{array}$ & $\begin{array}{r}7 \\
8 \\
9 \\
10 \\
11\end{array}$ & $\begin{array}{l}78 \\
78 \\
78 \\
78 \\
78\end{array}$ & $\begin{array}{l}39 . \\
41 . \\
49 . \\
53 . \\
53 .\end{array}$ & $\begin{array}{r}8 . \\
8 . \\
11 . \\
21 . \\
27 .\end{array}$ & $\begin{array}{l}28 . \\
290^{\circ} \\
450^{\circ} \\
51 . \\
49 .\end{array}$ & $\begin{array}{l}24 . \\
29^{\circ} \\
31 . \\
36 . \\
37^{\circ}\end{array}$ \\
\hline $\begin{array}{l}132 \\
133 \\
134 \\
135 \\
136\end{array}$ & $\begin{array}{l}5 \\
5 \\
5 \\
5 \\
5\end{array}$ & $\begin{array}{l}12 \\
13 \\
14 \\
15 \\
16\end{array}$ & $\begin{array}{l}78 \\
78 \\
78 \\
78 \\
78\end{array}$ & $\begin{array}{l}60 . \\
60 . \\
66 . \\
69 . \\
67 .\end{array}$ & $\begin{array}{l}21 . \\
29 . \\
27 . \\
32 . \\
28 .\end{array}$ & $\begin{array}{l}38 . \\
57 . \\
63 \\
66 . \\
62 .\end{array}$ & $\begin{array}{l}28 . \\
40 \\
41 . \\
44{ }^{\circ} \\
40\end{array}$ \\
\hline $\begin{array}{l}137 \\
138 \\
139 \\
140 \\
141\end{array}$ & $\begin{array}{l}5 \\
5 \\
5 \\
5 \\
5\end{array}$ & $\begin{array}{l}17 \\
18 \\
19 \\
20 \\
21\end{array}$ & $\begin{array}{l}78 \\
78 \\
78 \\
78 \\
78\end{array}$ & $\begin{array}{l}62 . \\
50 . \\
61 . \\
61 . \\
49 .\end{array}$ & $\begin{array}{l}22 . \\
27^{\circ} \\
180^{\circ} \\
34^{\circ} \\
28^{\circ}\end{array}$ & $\begin{array}{l}35 \\
51 \\
61 \\
46 \\
47\end{array}$ & $\begin{array}{l}32 \\
34 \\
40 \\
40 \\
39\end{array}$ \\
\hline
\end{tabular}


May-September 1977, 1978, and 1979--Continued

\begin{tabular}{|c|c|c|c|c|c|c|c|}
\hline $\begin{array}{l}\text { PAN } \\
\text { MAX } \\
\text { (F) }\end{array}$ & $\begin{array}{l}\text { WATER } \\
\text { MIN } \\
\text { (F) }\end{array}$ & $\begin{array}{l}\text { PRECIP } \\
\text { (IN.) }\end{array}$ & $\begin{array}{l}\text { WIND } \\
\text { (MI./DAY) }\end{array}$ & $\begin{array}{l}\text { PAN } \\
\text { EVAP } \\
\text { (IN.) }\end{array}$ & $\begin{array}{l}\text { DAI } \\
\text { IEMP } \\
\text { (F) }\end{array}$ & $\begin{array}{l}\text { Y AVG } \\
\text { REL HUM } \\
\text { (PERCENT) }\end{array}$ & $\begin{array}{l}\text { SOLAR } \\
\text { RAD } \\
(L Y,)\end{array}$ \\
\hline $\begin{array}{l}66 . \\
64 . \\
57 . \\
55 . \\
51 .\end{array}$ & $\begin{array}{l}38 . \\
37 . \\
38 . \\
30 \\
36 .\end{array}$ & $\begin{array}{r}0.00 \\
0.00 \\
.03 \\
0.00 \\
.25\end{array}$ & $\begin{array}{r}85 . \\
83 . \\
111 . \\
102 . \\
42 .\end{array}$ & $\begin{array}{l}.27 \\
.27 \\
.23 \\
.26 \\
.38\end{array}$ & $\begin{array}{l}48 \\
48 \\
44 \\
36 \\
29\end{array}$ & $\begin{array}{l}51 . \\
44 . \\
47^{\circ} \\
34 . \\
83 .\end{array}$ & $\begin{array}{l}373^{\circ} \\
350^{\circ} \\
325^{\circ} \\
465^{\circ} \\
193\end{array}$ \\
\hline $\begin{array}{l}50 . \\
54 . \\
53 . \\
43 . \\
41 .\end{array}$ & $\begin{array}{l}31 . \\
36 . \\
32 . \\
31 . \\
31 .\end{array}$ & $\begin{array}{r}.02 \\
0.00 \\
.06 \\
.08 \\
.32\end{array}$ & $\begin{array}{r}71 . \\
88 . \\
129 . \\
93 . \\
165^{\circ}\end{array}$ & $\begin{array}{l}.09 \\
.12 \\
.13 \\
.08 \\
\star * \star\end{array}$ & $\begin{array}{r}27 \\
32 \\
4 * 4 \\
* * 4 \\
* \# 4\end{array}$ & 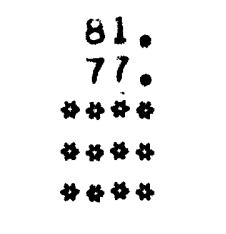 & $\begin{array}{l}464^{\circ} \\
559^{\circ} \\
397^{\circ} \\
288 . \\
154^{\circ}\end{array}$ \\
\hline $\begin{array}{l}40 \\
40 . \\
390^{\circ} \\
440^{\circ} \\
46 .\end{array}$ & $\begin{array}{l}4 \% \\
32 . \\
32 \\
31 \\
32 .\end{array}$ & $\begin{array}{r}.02 \\
.02 \\
0.00 \\
0.00 \\
0.00\end{array}$ & $\begin{array}{r}90 . \\
94{ }^{\circ} \\
106 . \\
770^{\circ} \\
143 .\end{array}$ & $\begin{array}{l}\star \star \star \star \\
\star \star \star \star \\
\star \star \star \star \\
\star \star \star \\
.13\end{array}$ & $\begin{array}{r}\# * 4 \\
* * * 4 \\
* * * 4 \\
* * 4 \\
41 .\end{array}$ & 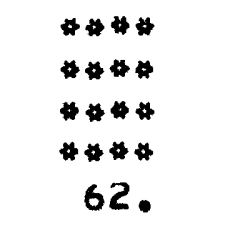 & $\begin{array}{l}602 . \\
5590^{\circ} \\
701 . \\
6970^{\circ} \\
474 .\end{array}$ \\
\hline $\begin{array}{l}55 . \\
67 . \\
69 . \\
71 . \\
70 .\end{array}$ & $\begin{array}{l}33 . \\
35 . \\
37 \\
37 \\
37\end{array}$ & $\begin{array}{l}.05 \\
0.00 \\
0.00 \\
0.00 \\
0.00\end{array}$ & $\begin{array}{l}207 . \\
111 . \\
94 . \\
1170 \\
115 .\end{array}$ & $\begin{array}{r}.27 \\
.25 \\
.33 \\
.38 \\
.46\end{array}$ & $\begin{array}{l}35 . \\
46 \\
48 \\
51 \\
50\end{array}$ & $\begin{array}{l}32 . \\
20 . \\
27 . \\
32 . \\
28 .\end{array}$ & $\begin{array}{l}785 . \\
700 . \\
710 . \\
696 . \\
671 .\end{array}$ \\
\hline $\begin{array}{l}60 . \\
60 . \\
670^{\circ} \\
67 . \\
53 .\end{array}$ & $\begin{array}{l}37 . \\
310^{\circ} \\
34 . \\
40 \\
37 .\end{array}$ & $\begin{array}{l}0.00 \\
0.00 \\
0.00 \\
.01 \\
.05\end{array}$ & $\begin{array}{r}132 . \\
204^{\circ} \\
93 . \\
83 . \\
89 .\end{array}$ & $\begin{array}{l}.24 \\
.26 \\
.26 \\
.19 \\
.09\end{array}$ & $\begin{array}{r}38 \\
\# \# * \\
4 \% \# \\
41 . \\
40\end{array}$ & $\begin{array}{r}58 . \\
* 4 * 4 \\
4 * 4 \\
91 . \\
72 .\end{array}$ & $\begin{array}{l}441 . \\
769^{\circ} \\
7030^{\circ} \\
2490^{\circ} \\
320 .\end{array}$ \\
\hline
\end{tabular}


Table 5.--Climatic data, Fairplay station,

\begin{tabular}{|c|c|c|c|c|c|c|c|}
\hline $\begin{array}{r}\text { DAY } \\
\text { OF } \\
\text { YEAR }\end{array}$ & MONTH & DAY & YEAR & $\begin{array}{l}\text { TEMF } \\
\text { MAX } \\
\text { (F) }\end{array}$ & $\begin{array}{l}\text { TURE } \\
\text { MIN } \\
\text { (F) }\end{array}$ & $\begin{array}{l}\text { DRY } \\
\text { BULB } \\
\text { (F) }\end{array}$ & $\begin{array}{l}\text { WET } \\
\text { BULB } \\
\text { (F) }\end{array}$ \\
\hline $\begin{array}{l}142 \\
143 \\
144 \\
145 \\
146\end{array}$ & $\begin{array}{l}5 \\
5 \\
5 \\
5 \\
5\end{array}$ & $\begin{array}{l}22 \\
23 \\
24 \\
25 \\
26\end{array}$ & $\begin{array}{l}78 \\
78 \\
78 \\
78 \\
78\end{array}$ & $\begin{array}{l}60 . \\
63 . \\
60 . \\
59 . \\
59 .\end{array}$ & $\begin{array}{l}30 . \\
26 . \\
25 . \\
25 . \\
26 .\end{array}$ & $\begin{array}{l}58 \\
55 \\
56 \\
59 \\
55\end{array}$ & $\begin{array}{l}42 . \\
40 \\
37 \\
39 . \\
39 .\end{array}$ \\
\hline $\begin{array}{l}147 \\
148 \\
149 \\
150 \\
151\end{array}$ & $\begin{array}{l}5 \\
5 \\
5 \\
5 \\
5\end{array}$ & $\begin{array}{l}27 \\
28 \\
29 \\
30 \\
31\end{array}$ & $\begin{array}{l}78 \\
78 \\
18 \\
78 \\
78\end{array}$ & $\begin{array}{l}55 . \\
51 . \\
59 . \\
61 . \\
60 .\end{array}$ & $\begin{array}{l}30 . \\
24 . \\
27 . \\
26 . \\
32 .\end{array}$ & $\begin{array}{l}45 . \\
47 \\
57 \\
59 \\
58\end{array}$ & $\begin{array}{l}35 . \\
36 \\
37 \\
39 \\
38\end{array}$ \\
\hline $\begin{array}{l}152 \\
153 \\
154 \\
155 \\
156\end{array}$ & $\begin{array}{l}6 \\
6 \\
6 \\
6 \\
6\end{array}$ & $\begin{array}{l}1 \\
2 \\
3 \\
4 \\
5\end{array}$ & $\begin{array}{l}78 \\
78 \\
78 \\
78 \\
78\end{array}$ & $\begin{array}{l}58 . \\
62 . \\
62 . \\
60 . \\
51 .\end{array}$ & $\begin{array}{l}35 . \\
35 . \\
37 . \\
25 . \\
30 .\end{array}$ & $\begin{array}{l}49 . \\
550 \\
60 \\
37 \\
40\end{array}$ & $\begin{array}{l}42 . \\
44{ }^{\circ} \\
40 \\
36 \\
38\end{array}$ \\
\hline $\begin{array}{l}157 \\
158 \\
159 \\
160 \\
161\end{array}$ & $\begin{array}{l}6 \\
6 \\
6 \\
6 \\
6\end{array}$ & $\begin{array}{r}6 \\
7 \\
8 \\
9 \\
10\end{array}$ & $\begin{array}{l}78 \\
78 \\
78 \\
78 \\
78\end{array}$ & $\begin{array}{l}56 . \\
58 . \\
58 . \\
68 . \\
69 .\end{array}$ & $\begin{array}{l}26 . \\
32 . \\
30 . \\
28 . \\
36 .\end{array}$ & $\begin{array}{l}55 \\
45 \\
55 \\
66 \\
64 .\end{array}$ & $\begin{array}{l}38 \\
38 \\
40 \\
46 \\
41\end{array}$ \\
\hline $\begin{array}{l}162 \\
163 \\
164 \\
165 \\
166\end{array}$ & $\begin{array}{l}6 \\
6 \\
6 \\
6 \\
6\end{array}$ & $\begin{array}{l}11 \\
12 \\
13 \\
14 \\
15\end{array}$ & $\begin{array}{l}78 \\
78 \\
78 \\
78 \\
78\end{array}$ & $\begin{array}{l}65 \\
70 . \\
70 . \\
71 . \\
72 .\end{array}$ & $\begin{array}{l}44 . \\
32 \\
34 \\
35 . \\
34 .\end{array}$ & $\begin{array}{l}60 . \\
67 \\
67 \\
66 . \\
68 .\end{array}$ & $\begin{array}{l}43 . \\
44 \circ^{\circ} \\
43{ }^{\circ} \\
440^{\circ}\end{array}$ \\
\hline
\end{tabular}


May-September 1977, 1978, and 1979--Continued

\begin{tabular}{|c|c|c|c|c|c|}
\hline $\begin{array}{l}\text { PAN } \\
\text { MAX } \\
\text { (F) }\end{array}$ & $\begin{array}{l}\text { ATER } \\
\text { MIN } \\
\text { (F) }\end{array}$ & $\begin{array}{l}\text { PHECIP } \\
(I N \cdot)\end{array}$ & $\begin{array}{l}\text { WIND } \\
\text { (MI./DAY) }\end{array}$ & $\begin{array}{l}\text { PAN } \\
\text { EVAP } \\
(I N \cdot)\end{array}$ & $\begin{array}{l}\text { DAILY AVG } \\
\text { TEMP REL HUM } \\
\text { (F) }\end{array}$ \\
\hline
\end{tabular}

$\begin{array}{llllllll}66 . & 37 . & 0.00 & 115 . & .28 & 48 . & 39 . & 621 . \\ 65 . & 37 \circ & 0.00 & 118 . & .32 & 44 . & 43 . & 554 . \\ 66 . & 37 . & 0.00 & 123 . & .38 & 44 . & 34 . & 747 . \\ 62 . & 36 . & 0.00 & 111 . & .30 & 44 . & 21 . & 595 . \\ 61 . & 36 . & 0.00 & 114 . & .32 & 43 . & 36 . & 539 .\end{array}$

\begin{tabular}{|c|c|c|c|c|c|c|c|}
\hline $\begin{array}{l}60 . \\
60 . \\
67 . \\
65 . \\
62 .\end{array}$ & $\begin{array}{l}38 . \\
37 \circ^{\circ} \\
37{ }^{\circ} \\
37 . \\
38 .\end{array}$ & $\begin{array}{l}.12 \\
0.00 \\
0.00 \\
0.00 \\
0.00\end{array}$ & $\begin{array}{l}76 . \\
104 \\
112 . \\
177 \\
133 .\end{array}$ & $\begin{array}{r}.13 \\
.19 \\
.31 \\
.46 \\
.31\end{array}$ & $\begin{array}{l}38 . \\
37 . \\
42 . \\
47 . \\
43 .\end{array}$ & $\begin{array}{l}74 . \\
53 . \\
28 . \\
25 . \\
59 .\end{array}$ & $\begin{array}{l}347 . \\
572 . \\
723 . \\
678 . \\
654 .\end{array}$ \\
\hline
\end{tabular}

\begin{tabular}{|c|c|c|c|c|c|c|c|}
\hline $\begin{array}{l}62 . \\
66 . \\
65 . \\
63 . \\
55 .\end{array}$ & $\begin{array}{l}38 . \\
39 . \\
42 . \\
38 . \\
36 .\end{array}$ & $\begin{array}{r}.05 \\
0.00 \\
.03 \\
.50 \\
.33\end{array}$ & $\begin{array}{r}143 \\
73 \\
86 \\
68 \\
59\end{array}$ & $\begin{array}{r}.21 \\
.15 \\
.27 \\
.16 \\
.07\end{array}$ & $\begin{array}{l}40 \\
43 \\
45 . \\
36 \\
37 .\end{array}$ & $\begin{array}{l}85 . \\
70 . \\
52 . \\
90 . \\
85 .\end{array}$ & $\begin{array}{l}433 . \\
513 . \\
480 \\
225 \\
377^{\circ}\end{array}$ \\
\hline
\end{tabular}

\begin{tabular}{|c|c|c|c|c|c|c|c|}
\hline $\begin{array}{l}65 . \\
64 . \\
67 . \\
71 . \\
69 .\end{array}$ & $\begin{array}{l}38 . \\
38 \\
37 \\
38 \\
41 .\end{array}$ & $\begin{array}{r}.06 \\
.09 \\
.07 \\
0.00 \\
0.00\end{array}$ & $\begin{array}{r}86 . \\
96 . \\
88 . \\
103 . \\
142 .\end{array}$ & $\begin{array}{r}.25 \\
.25 \\
.24 \\
.35 \\
.45\end{array}$ & $\begin{array}{l}41 . \\
41 . \\
44 . \\
50 \\
55 .\end{array}$ & $\begin{array}{l}51 . \\
63 . \\
52 . \\
43 . \\
22 .\end{array}$ & $\begin{array}{r}734 . \\
431 . \\
* 4 \% \\
7 * 4 \\
706 .\end{array}$ \\
\hline
\end{tabular}

\begin{tabular}{|c|c|c|c|c|c|c|}
\hline $\begin{array}{l}71 . \\
72 . \\
72 . \\
73 . \\
71 .\end{array}$ & $\begin{array}{l}41 . \\
40 . \\
410^{\circ} \\
39 . \\
42 .\end{array}$ & $\begin{array}{l}0.00 \\
0.00 \\
0.00 \\
0.00 \\
0.00\end{array}$ & $\begin{array}{r}130 . \\
80 . \\
95 . \\
101 . \\
143 .\end{array}$ & $\begin{array}{l}.46 \\
.31 \\
.36 \\
.45 \\
.48\end{array}$ & $\begin{array}{l}53 . \\
49^{\circ} \\
54 . \\
54 . \\
57^{\circ}\end{array}$ & $\begin{array}{l}16 . \\
59 . \\
42 . \\
27 . \\
25 .\end{array}$ \\
\hline
\end{tabular}


Table 5.--Climatic data, Fairplay station,

DAY MONTH
OF
YEAR

DAY YEAR

TEMPERATURE

MAX

(F)

MIN

DRY

WET

167

168

169

170

171

$\begin{array}{ll}6 & 16 \\ 6 & 17 \\ 6 & 18 \\ 6 & 19 \\ 6 & 20\end{array}$

78

78

78

78

78

(F)

BULB

BULB

(F)

(F)

172

173

174

175

176

$\begin{array}{ll}6 & 21 \\ 6 & 22 \\ 6 & 23 \\ 6 & 24 \\ 6 & 25\end{array}$

78

78

78

78

78

$\begin{array}{ll}71 . & 31 . \\ 70 . & 33 . \\ 69 . & 30 . \\ 68 . & 36 . \\ 69 . & 28 .\end{array}$

68.

61.

66.

64.

66.

42.

40 .

42.

42 .

44.

177

178

179

180

181

$\begin{array}{ll}6 & 26 \\ 6 & 27 \\ 6 & 28 \\ 6 & 29 \\ 6 & 30\end{array}$

78

78

78

78

73.

33.

36.

69.

44.

76.

35.

75.

47 .

74.

46.

47.

13 .

49.

74.

48.

64.

42 .

182

183

184

185

186

78

70.30 .

68 .

72.

38.

43.

65.

46.

67.

42 .

67.

58.

61 .

53.

56.

43.

49.

48 .

50 .

46.

187

188

189

78

78

69.

34.

36 .

75.

36.

78

72.

37.

73.

33.

65.

71 .

46.

71 .

46.

69.

43.

78

71.

42.

43.

190

191

7
7
7
7
7

78

78

71.

16.

38.

34.

15.

38.

78

13.

41 .

67.

37.

66.

74.

41.

73.

46.

46.

54 .

46.

10

78

58 .

49. 
May-September 1977, 1978, and 1979--Continued

\begin{tabular}{|c|c|c|c|c|c|c|c|}
\hline $\begin{array}{l}\text { PAN } \\
\text { MAX } \\
\text { (F) }\end{array}$ & $\begin{array}{l}\text { WATER } \\
\text { MIN } \\
\text { (F) }\end{array}$ & $\begin{array}{l}\text { PRECIP } \\
(I N \cdot)\end{array}$ & $\begin{array}{l}\text { WIND } \\
\text { (MI./DAY) }\end{array}$ & $\begin{array}{l}\text { PAN } \\
\text { EVAP } \\
\text { (IN.) }\end{array}$ & $\begin{array}{l}\text { DAI } \\
\text { TEMP } \\
\text { (F) }\end{array}$ & $\begin{array}{l}\text { Y AVG } \\
\text { REL HUM } \\
\text { (PERCENT) }\end{array}$ & $\begin{array}{l}\text { SOLAR } \\
R A D \\
\left(L Y_{\cdot}\right)\end{array}$ \\
\hline $\begin{array}{l}71 . \\
71 . \\
71 . \\
72 . \\
72 .\end{array}$ & $\begin{array}{l}38 . \\
38 . \\
38 . \\
41 . \\
38 .\end{array}$ & $\begin{array}{l}0.00 \\
0.00 \\
0.00 \\
0.00 \\
0.00\end{array}$ & $\begin{array}{r}96 . \\
137 . \\
89 . \\
137 . \\
99 .\end{array}$ & $\begin{array}{l}.33 \\
.51 \\
.36 \\
.48 \\
.41\end{array}$ & $\begin{array}{l}54 . \\
52 . \\
51 . \\
56 . \\
50 .\end{array}$ & $\begin{array}{l}19 . \\
190^{\circ} \\
37^{\circ} \\
210^{\circ} \\
43 .\end{array}$ & $\begin{array}{l}778 \\
765 . \\
700 \\
746 . \\
751\end{array}$ \\
\hline $\begin{array}{l}73 . \\
77 . \\
76 . \\
73 . \\
72 .\end{array}$ & $\begin{array}{l}42 . \\
39 . \\
430^{\circ} \\
440^{\circ} \\
46 .\end{array}$ & $\begin{array}{l}0.00 \\
0.00 \\
0.00 \\
0.00 \\
0.00\end{array}$ & $\begin{array}{r}89 . \\
99 . \\
88^{\circ} \\
127^{\circ} \\
152 .\end{array}$ & $\begin{array}{l}.43 \\
.44 \\
.48 \\
.50 \\
.50\end{array}$ & $\begin{array}{l}54 . \\
59 . \\
60 . \\
61 . \\
60 .\end{array}$ & $\begin{array}{l}38 . \\
230 \\
23 . \\
230 \\
20\end{array}$ & $\begin{array}{l}739 . \\
665 \\
723 \\
625 \\
621 .\end{array}$ \\
\hline $\begin{array}{l}70 . \\
69 . \\
71 . \\
68 . \\
73 .\end{array}$ & $\begin{array}{l}38 . \\
45 . \\
46 . \\
47 \\
44 .\end{array}$ & $\begin{array}{r}0.00 \\
.03 \\
.01 \\
.07 \\
.10\end{array}$ & $\begin{array}{r}115 . \\
67 . \\
58 . \\
67 . \\
116 .\end{array}$ & $\begin{array}{l}.48 \\
.25 \\
.23 \\
.18 \\
.32\end{array}$ & $\begin{array}{l}52 . \\
51 . \\
56 . \\
52 . \\
54 .\end{array}$ & $\begin{array}{l}32 . \\
78 . \\
56 . \\
80 . \\
59 .\end{array}$ & $\begin{array}{l}744 \\
420^{\circ} \\
367^{\circ} \\
344 \\
706\end{array}$ \\
\hline $\begin{array}{l}69 . \\
750^{\circ} \\
70 . \\
71 . \\
74 .\end{array}$ & $\begin{array}{l}40 . \\
39 . \\
39 . \\
38 . \\
38 .\end{array}$ & $\begin{array}{l}0.00 \\
0.00 \\
0.00 \\
0.00 \\
0.00\end{array}$ & $\begin{array}{r}51 . \\
83 . \\
106 . \\
136 . \\
82 .\end{array}$ & $\begin{array}{l}.22 \\
.38 \\
.46 \\
.53 \\
.40\end{array}$ & $\begin{array}{l}53 . \\
57 \\
55 \\
55 \\
54\end{array}$ & $\begin{array}{l}45 . \\
330^{\circ} \\
170^{\circ} \\
130^{\circ} \\
17 .\end{array}$ & $\begin{array}{l}392 . \\
621 . \\
644 . \\
744 . \\
606 .\end{array}$ \\
\hline $\begin{array}{l}74 . \\
750^{\circ} \\
74 . \\
72 . \\
62 .\end{array}$ & $\begin{array}{l}37 . \\
420^{\circ} \\
430^{\circ} \\
44{ }^{\circ} \\
45\end{array}$ & $\begin{array}{r}0.00 \\
0.00 \\
0.00 \\
0.00 \\
.02\end{array}$ & $\begin{array}{r}100 . \\
82 . \\
123 . \\
93 . \\
71 .\end{array}$ & $\begin{array}{l}.42 \\
.39 \\
.54 \\
.36 \\
.19\end{array}$ & $\begin{array}{l}54 . \\
55 . \\
59 . \\
55 . \\
50 .\end{array}$ & $\begin{array}{l}22 . \\
470^{\circ} \\
24 \circ^{\circ} \\
44{ }^{\circ} \\
72 .\end{array}$ & $\begin{array}{l}665 . \\
729 . \\
700 . \\
288 . \\
272 .\end{array}$ \\
\hline
\end{tabular}


Table 5.--Climatic data, Faipplay station,

\begin{tabular}{|c|c|c|c|c|c|c|c|}
\hline $\begin{array}{r}\text { DAY } \\
\text { OF } \\
\text { YEAR }\end{array}$ & MONTH & DAY & YEAR & $\begin{array}{l}\text { TEMF } \\
\text { MAX } \\
\text { (F) }\end{array}$ & $\begin{array}{l}\text { TURE } \\
\text { MIN } \\
\text { (F) }\end{array}$ & $\begin{array}{l}\text { DRY } \\
\text { BULR } \\
\text { (F) }\end{array}$ & $\begin{array}{l}\text { WET } \\
\text { BULB } \\
\text { (F) }\end{array}$ \\
\hline $\begin{array}{l}192 \\
193 \\
194 \\
195 \\
196\end{array}$ & $\begin{array}{l}7 \\
7 \\
7 \\
7 \\
7\end{array}$ & $\begin{array}{l}11 \\
12 \\
13 \\
14 \\
15\end{array}$ & $\begin{array}{l}78 \\
78 \\
78 \\
78 \\
78\end{array}$ & $\begin{array}{l}70 . \\
74 . \\
770^{\circ} \\
79 . \\
75 .\end{array}$ & $\begin{array}{l}38 . \\
37 . \\
41 . \\
36 \\
41 .\end{array}$ & $\begin{array}{l}63 . \\
71 . \\
73 . \\
72 . \\
52 .\end{array}$ & $\begin{array}{l}47 \\
47 \\
48 \\
46 \\
47\end{array}$ \\
\hline $\begin{array}{l}197 \\
198 \\
199 \\
200 \\
201\end{array}$ & $\begin{array}{l}7 \\
7 \\
7 \\
7 \\
7\end{array}$ & $\begin{array}{l}16 \\
17 \\
18 \\
19 \\
20\end{array}$ & $\begin{array}{l}78 \\
78 \\
78 \\
78 \\
78\end{array}$ & $\begin{array}{l}15 . \\
75 . \\
75 . \\
73 . \\
73 .\end{array}$ & $\begin{array}{l}39 . \\
46 . \\
43 . \\
38 . \\
35 .\end{array}$ & $\begin{array}{l}58 . \\
65 . \\
69 . \\
63 . \\
62 .\end{array}$ & $\begin{array}{l}53 . \\
46 . \\
50 . \\
49 . \\
50 .\end{array}$ \\
\hline $\begin{array}{l}202 \\
203 \\
204 \\
205 \\
206\end{array}$ & $\begin{array}{l}7 \\
7 \\
7 \\
7 \\
7\end{array}$ & $\begin{array}{l}21 \\
22 \\
23 \\
24 \\
25\end{array}$ & $\begin{array}{l}78 \\
78 \\
78 \\
78 \\
78\end{array}$ & $\begin{array}{l}74 . \\
73 . \\
72 . \\
76 . \\
76 .\end{array}$ & $\begin{array}{l}40 . \\
35 . \\
35 \\
38 . \\
37 .\end{array}$ & $\begin{array}{l}66 . \\
60 . \\
65 . \\
67 . \\
69 .\end{array}$ & $\begin{array}{l}46 . \\
51 \\
53 \\
47 \\
48\end{array}$ \\
\hline $\begin{array}{l}207 \\
208 \\
209 \\
210 \\
211\end{array}$ & $\begin{array}{l}7 \\
7 \\
7 \\
7 \\
7\end{array}$ & $\begin{array}{l}26 \\
27 \\
28 \\
29 \\
30\end{array}$ & $\begin{array}{l}78 \\
78 \\
78 \\
78 \\
78\end{array}$ & $\begin{array}{l}77 . \\
80 . \\
76 . \\
72 . \\
71 .\end{array}$ & $\begin{array}{l}39 . \\
38 . \\
40 . \\
42 . \\
40 .\end{array}$ & $\begin{array}{l}69 . \\
69 . \\
67 . \\
60 . \\
63 .\end{array}$ & $\begin{array}{l}51 . \\
48 . \\
51 . \\
50 . \\
51 .\end{array}$ \\
\hline $\begin{array}{l}212 \\
213 \\
214 \\
215 \\
216\end{array}$ & $\begin{array}{l}7 \\
8 \\
8 \\
8 \\
8\end{array}$ & $\begin{array}{r}31 \\
1 \\
2 \\
3 \\
4\end{array}$ & $\begin{array}{l}78 \\
78 \\
78 \\
78 \\
78\end{array}$ & $\begin{array}{l}73 . \\
11 . \\
12 . \\
62 . \\
67 .\end{array}$ & $\begin{array}{l}37 . \\
35 . \\
36 . \\
37 . \\
34 .\end{array}$ & $\begin{array}{l}59 . \\
50 . \\
49 . \\
55 . \\
53 .\end{array}$ & $\begin{array}{l}47 \\
46 . \\
47 \\
48 \\
45 .\end{array}$ \\
\hline
\end{tabular}


May-September 1977, 1978, and 1979--Continued

\begin{tabular}{|c|c|c|c|c|c|c|c|}
\hline $\begin{array}{l}\text { PAN } \\
\text { MAX } \\
\text { (F) }\end{array}$ & $\begin{array}{l}\text { WATER } \\
\text { MIN } \\
\text { (F) }\end{array}$ & $\begin{array}{l}\text { PRECIP } \\
\left(I N_{\bullet}\right)\end{array}$ & $\begin{array}{l}\text { WIND } \\
\text { (MI./DAY) }\end{array}$ & $\begin{array}{l}\text { PAN } \\
\text { EVAP } \\
\text { (IN.) }\end{array}$ & $\begin{array}{l}\text { DAI } \\
\text { IEMP } \\
\text { (F) }\end{array}$ & $\begin{array}{l}\text { Y AVG } \\
\text { REL HUM } \\
\text { (PERCENT) }\end{array}$ & $\begin{array}{l}\text { SOLAR } \\
\text { RAD } \\
(L Y .)\end{array}$ \\
\hline $\begin{array}{l}69 . \\
76 . \\
77 . \\
76 . \\
69 .\end{array}$ & $\begin{array}{l}43 . \\
42 . \\
46 . \\
46 . \\
45\end{array}$ & $\begin{array}{r}0.00 \\
.05 \\
0.00 \\
0.00 \\
.03\end{array}$ & $\begin{array}{l}95 . \\
81 . \\
73 . \\
71 . \\
80\end{array}$ & $\begin{array}{r}.26 \\
.32 \\
.37 \\
.38 \\
.29\end{array}$ & $\begin{array}{l}54 . \\
56 . \\
60 . \\
62 . \\
56 .\end{array}$ & $\begin{array}{l}60 . \\
450^{\circ} \\
440^{\circ} \\
350^{\circ} \\
50 .\end{array}$ & $\begin{array}{l}436 . \\
618^{\circ} \\
660^{\circ} \\
444 . \\
210^{\circ}\end{array}$ \\
\hline $\begin{array}{l}71 . \\
77 . \\
75 \\
70 \\
69 .\end{array}$ & $\begin{array}{l}42 . \\
46 . \\
470^{\circ} \\
420^{\circ} \\
42 .\end{array}$ & $\begin{array}{r}.02 \\
0.00 \\
0.00 \\
0.00 \\
.10\end{array}$ & $\begin{array}{r}90 . \\
129 . \\
11 . \\
92 . \\
68 .\end{array}$ & $\begin{array}{r}.23 \\
.39 \\
.31 \\
.41 \\
.30\end{array}$ & $\begin{array}{l}55 . \\
58 . \\
58 . \\
56 . \\
54 .\end{array}$ & $\begin{array}{l}64 . \\
450^{\circ} \\
42 . \\
470^{\circ} \\
71 .\end{array}$ & $\begin{array}{l}383 \\
596 \\
665 \\
387 \\
380\end{array}$ \\
\hline $\begin{array}{l}72 . \\
73 \\
77 \\
75 \circ \\
71\end{array}$ & $\begin{array}{l}44 . \\
460^{\circ} \\
460^{\circ} \\
43^{\circ} \\
41 .\end{array}$ & $\begin{array}{r}0.00 \\
0.00 \\
.16 \\
.01 \\
0.00\end{array}$ & $\begin{array}{l}97 . \\
58 . \\
58 . \\
65 . \\
94 .\end{array}$ & $\begin{array}{l}.36 \\
.27 \\
.38 \\
.30 \\
.38\end{array}$ & $\begin{array}{l}56 . \\
53 . \\
55 . \\
56 . \\
58 .\end{array}$ & $\begin{array}{l}57 . \\
62 . \\
59 . \\
50 . \\
32 .\end{array}$ & $\begin{array}{l}632 . \\
467 . \\
636 . \\
520 \\
393 .\end{array}$ \\
\hline $\begin{array}{l}750^{\circ} \\
790^{\circ} \\
71^{\circ} \\
670^{\circ} \\
73 .\end{array}$ & $\begin{array}{l}430^{\circ} \\
45^{\circ} \\
44 \circ^{\circ} \\
45^{\circ} \\
430^{\circ}\end{array}$ & $\begin{array}{r}0.00 \\
.01 \\
0.00 \\
.19 \\
.02\end{array}$ & $\begin{array}{l}93 . \\
58 . \\
72 . \\
81 . \\
74 .\end{array}$ & $\begin{array}{l}.40 \\
.36 \\
.26 \\
.32 \\
.26\end{array}$ & $\begin{array}{l}58 . \\
59 . \\
58 . \\
550^{\circ} \\
55 .\end{array}$ & $\begin{array}{l}42 . \\
480^{\circ} \\
500^{\circ} \\
730^{\circ} \\
54 .\end{array}$ & $\begin{array}{l}625 . \\
487 . \\
302 . \\
455 . \\
501 .\end{array}$ \\
\hline $\begin{array}{l}72 . \\
70 \\
72 . \\
65 \\
68\end{array}$ & $\begin{array}{l}49 . \\
42 . \\
40 . \\
46 . \\
44 .\end{array}$ & $\begin{array}{r}0.00 \\
.03 \\
.01 \\
.06 \\
0.00\end{array}$ & $\begin{array}{r}69 . \\
114 . \\
101 . \\
50 . \\
76 .\end{array}$ & $\begin{array}{l}.26 \\
.19 \\
.38 \\
.15 \\
.26\end{array}$ & $\begin{array}{l}\$ 4 \% \\
51 . \\
54 . \\
48 \\
50\end{array}$ & $\begin{array}{l}64 . \\
69 . \\
59 . \\
80 . \\
62 .\end{array}$ & $\begin{array}{l}405 \\
469 \\
554 \\
392 \\
431\end{array}$ \\
\hline
\end{tabular}


Table 5.--Climatic data, Fairplay station,

$\begin{array}{cc}\text { DAY } & \text { MONTH } \\ \text { OF } & \\ \text { YEAR } & \\ & \\ & \\ 217 & 8 \\ 218 & 8 \\ 219 & 8 \\ 220 & 8 \\ 221 & 8\end{array}$

222

223

224

225

226

227

228

229

230

231

232

233

234

235

236

237

238

239

240

241

5

6

7

8

9

YEAR

TEMP
MAX
(F)

(F)

13.

16.

69.

67.

69.

10

11

12

13

14

8

8

8

8

8

8

8

8

8

8

8

15

16

17

is

19

20

21

22

23

24

$8 \quad 25$

826

$8 \quad 27$

828

$8 \quad 29$
78

78

78

78

78

78

78

78

78

78

78

78

78

78

78

78

78

78

78

78

78

78

78

78
71.

73.

69.

63.

68.

12.

12.

64.

10 .

71.

11.

64 .

13.

68.

65.

68 .

70 .

10.

67.
32.

37.

37.

37.

33.

34.

38 .

40 .

39.

35.

34.

38 .

45.

31 .

23.

33.

36.

36.

35.

40 .

45.

33.

35.

31 .

34.
DRY

RULB

(F)

WET

BULB

(F)

70.

59.

60.

58.

56.

60.

60 .

49.

57.

37.

66.

68.

63.

55.

65.

64.

51 .

61.

65.

61 .

61.

64.

67.

56.

54 .
47. 48 . 48 . 48 .

47. 49. 40 . 48. 37.

46. 45. 43. 41 . 45.

46. 48. 48. 47. 47.

49. 45. 45. 44. 43. 47. 
May-September 1977, 1978, and 1979--Continued

$\begin{array}{ccccccc}\text { PAN WATER } & \text { PRECIP } & \text { WIND } & \text { PAN } & \text { DAILY AVG } & \text { SOLAR } \\ \text { MAX } & \text { MIN } & & & \text { EVAP } & \text { TEMP } & \text { REL HUM RAD } \\ \text { (F) } & \text { (F) } & \text { (IN.) } & \text { (MI./DAY) } & \text { (IN.) } & \text { (F) } & \text { (PERCENT) (LY.) }\end{array}$

\begin{tabular}{|c|c|c|c|c|c|c|c|}
\hline $\begin{array}{l}76 . \\
75 \\
67 \\
64 \\
67\end{array}$ & $\begin{array}{l}38 . \\
41 . \\
43 . \\
39 . \\
41 .\end{array}$ & $\begin{array}{r}0.00 \\
0.00 \\
0.00 \\
0.00 \\
.01\end{array}$ & $\begin{array}{l}63 . \\
81 . \\
97 . \\
70 . \\
69 .\end{array}$ & $\begin{array}{r}.29 \\
.34 \\
.29 \\
.22 \\
.22\end{array}$ & $\begin{array}{r}52 . \\
54 . \\
52 . \\
50 .\end{array}$ & $\begin{array}{r}42 \\
45 \\
61 . \\
67 .\end{array}$ & $\begin{array}{l}661 . \\
314 . \\
396 . \\
354 . \\
422 .\end{array}$ \\
\hline
\end{tabular}

$\begin{array}{rrrrrrrr}65 . & 39 . & 0.00 & 94 . & .26 & 52 . & 53 . & 399 . \\ 67 . & 39 . & 0.00 & 81 . & .23 & 53 . & 60 . & 327 . \\ 70 . & 43 . & .02 & 80 . & .25 & 54 . & 62 . & 380 . \\ 66 . & 44 . & .23 & 85 . & .23 & 51 . & 68 . & 475 . \\ 64 . & 41 . & .08 & 110 . & .26 & .44 . & 78 . & 423 .\end{array}$

$\begin{array}{llllllll}70 . & 36 . & .03 & 128 . & .28 & 49 . & 26 . & 642 . \\ 71 . & 38 . & 0.00 & 116 . & .42 & 55 . & 22 . & 636 . \\ 70 & 40 . & 0.00 & 144 . & .50 & 57 . & 20 . & 631 . \\ 65 . & 39 . & 0.00 & 103 . & .31 & 44 . & 53 . & 544 \\ 67 . & 37 . & 0.00 & 79 . & .27 & 46 . & 56 . & 418 .\end{array}$

$\begin{array}{rrrrrrrr}68 . & 38 . & 0.00 & 67 . & .24 & 50 . & 49 . & 361 . \\ 66 . & 42 . & .02 & 61 . & .22 & .40 & 55 . & 256 \\ 65 . & 45 . & .07 & 74 . & .16 & 52 . & 68 . & 325 \\ 74 . & 39 . & 0.00 & 78 . & .30 & 53 . & 48 . & 464 \\ 64 . & 40 . & 0.00 & 62 . & .19 & 53 . & 53 . & 317 \\ & & & & & & & \\ 64 . & 46 . & .09 & 85 . & .22 & 51 . & 76 . & 357 \\ 66 . & 40 . & 0.00 & 67 . & .21 & 52 . & 46 . & 389 \\ 70 . & 40 . & 0.00 & 85 . & .36 & 52 . & 31 . & 553 \\ 70 . & 37 . & 0.00 & 74 . & .32 & 48 . & 54 . & 485 \\ 64 . & 41 . & .06 & 70 . & .20 & 45 . & 71 . & 295\end{array}$




\begin{tabular}{|c|c|c|c|c|c|c|c|}
\hline & & & & & & & \\
\hline $\begin{array}{r}\text { DAY } \\
\text { OF } \\
\text { YEAR }\end{array}$ & MONTH & DAY & YEAR & $\begin{array}{l}\text { TEMP } \\
\text { MAX } \\
\text { (F) }\end{array}$ & $\begin{array}{l}\text { TURE } \\
\text { MIN } \\
\text { (F) }\end{array}$ & $\begin{array}{l}\text { DRY } \\
\text { BULB } \\
\text { (F) }\end{array}$ & $\begin{array}{l}\text { WET } \\
\text { BULB } \\
(F)\end{array}$ \\
\hline $\begin{array}{l}242 \\
243 \\
244 \\
245 \\
246\end{array}$ & $\begin{array}{l}8 \\
8 \\
9 \\
9 \\
9\end{array}$ & $\begin{array}{r}30 \\
31 \\
1 \\
2 \\
3\end{array}$ & $\begin{array}{l}78 \\
78 \\
78 \\
78 \\
78\end{array}$ & $\begin{array}{l}69 . \\
68 \\
71 \\
12 \\
72\end{array}$ & $\begin{array}{l}27 \\
31 . \\
31 . \\
37 \\
37\end{array}$ & $\begin{array}{l}64 . \\
57^{\circ} \\
65^{\circ} \\
44 . \\
68^{\circ}\end{array}$ & $\begin{array}{l}45 \\
45 \\
46 \\
40 \\
48\end{array}$ \\
\hline $\begin{array}{l}247 \\
248 \\
249 \\
250 \\
251\end{array}$ & $\begin{array}{l}9 \\
9 \\
9 \\
9 \\
9\end{array}$ & $\begin{array}{l}4 \\
5 \\
6 \\
7 \\
8\end{array}$ & $\begin{array}{l}78 \\
78 \\
78 \\
78 \\
78\end{array}$ & $\begin{array}{l}78 \\
77 \\
78 \\
74 \\
70\end{array}$ & $\begin{array}{l}37 \\
36 \\
36 \\
36 \\
31 .\end{array}$ & $\begin{array}{l}68 . \\
69 . \\
72 . \\
51 . \\
61 .\end{array}$ & $\begin{array}{l}40^{\circ} \\
50^{\circ} \\
52^{\circ} \\
40^{\circ} \\
45 .\end{array}$ \\
\hline $\begin{array}{l}252 \\
253 \\
254 \\
255 \\
256\end{array}$ & $\begin{array}{l}9 \\
9 \\
9 \\
9 \\
9\end{array}$ & $\begin{array}{l}9 \\
10 \\
11 \\
12 \\
13\end{array}$ & $\begin{array}{l}78 \\
78 \\
78 \\
78 \\
78\end{array}$ & $\begin{array}{l}71 . \\
67 . \\
63 . \\
59 . \\
57 .\end{array}$ & $\begin{array}{l}37 \\
35 \\
38 \\
39 \\
20\end{array}$ & $\begin{array}{l}46 . \\
60 . \\
52 . \\
51 . \\
54 .\end{array}$ & $\begin{array}{l}40 \\
460^{\circ} \\
37^{\circ} \\
370^{\circ} \\
40^{\circ}\end{array}$ \\
\hline $\begin{array}{l}257 \\
258 \\
259 \\
260 \\
261\end{array}$ & $\begin{array}{l}9 \\
9 \\
9 \\
9 \\
9\end{array}$ & $\begin{array}{l}14 \\
15 \\
16 \\
17 \\
18\end{array}$ & $\begin{array}{l}78 \\
78 \\
78 \\
78 \\
78\end{array}$ & $\begin{array}{l}66 . \\
71 . \\
68 . \\
61 . \\
59 .\end{array}$ & $\begin{array}{l}23 \\
29 \\
43 \\
34 \\
27\end{array}$ & $\begin{array}{l}60 \\
64{ }^{\circ} \\
60 \\
53 \\
46 .\end{array}$ & $\begin{array}{l}42 . \\
46 . \\
45 . \\
42 . \\
37\end{array}$ \\
\hline $\begin{array}{l}262 \\
263 \\
264 \\
265 \\
266\end{array}$ & $\begin{array}{l}9 \\
9 \\
9 \\
9 \\
9\end{array}$ & $\begin{array}{l}19 \\
20 \\
21 \\
22 \\
23\end{array}$ & $\begin{array}{l}78 \\
78 \\
78 \\
78 \\
78\end{array}$ & $\begin{array}{l}49 . \\
40 \\
55^{\circ} \\
65 \\
67^{\circ}\end{array}$ & $\begin{array}{l}26 \\
25 \\
11 \\
22 \\
28\end{array}$ & $\begin{array}{l}28 \\
36 \\
51 \\
58 \\
61\end{array}$ & $\begin{array}{l}28 . \\
32 \\
37 \\
43 \\
43 .\end{array}$ \\
\hline $\begin{array}{l}267 \\
268 \\
269 \\
270 \\
271\end{array}$ & $\begin{array}{l}9 \\
9 \\
9 \\
9 \\
9\end{array}$ & $\begin{array}{l}2.4 \\
25 \\
26 \\
27 \\
28\end{array}$ & $\begin{array}{l}78 \\
78 \\
78 \\
78 \\
78\end{array}$ & $\begin{array}{l}63 . \\
58 \\
66 \\
65 \\
65\end{array}$ & $\begin{array}{l}33 . \\
36 \\
30 \\
31 . \\
29\end{array}$ & $\begin{array}{l}46 . \\
48 . \\
61 . \\
57 . \\
60 .\end{array}$ & $\begin{array}{l}39 \\
41 \\
42 \\
42 \\
43\end{array}$ \\
\hline $\begin{array}{l}272 \\
273\end{array}$ & $\begin{array}{l}9 \\
9\end{array}$ & $\begin{array}{l}29 \\
30\end{array}$ & $\begin{array}{l}78 \\
78\end{array}$ & $\begin{array}{l}66 \\
66\end{array}$ & $\begin{array}{l}33 \\
24\end{array}$ & $\begin{array}{l}59 . \\
45 .\end{array}$ & $\begin{array}{l}39 . \\
33 .\end{array}$ \\
\hline
\end{tabular}


May-September 1977, 1978, and 1979--Continued

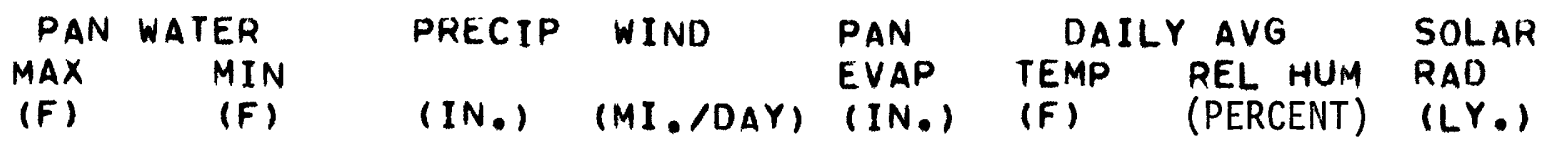

\begin{tabular}{|c|c|c|c|c|c|c|}
\hline $\begin{array}{l}68 \\
66 \\
72 \\
70 \\
75\end{array}$ & $\begin{array}{l}37 \\
39 \\
38 \\
39 \\
38\end{array}$ & $\begin{array}{r}0.00 \\
0.00 \\
0.00 \\
.03 \\
0.00\end{array}$ & $\begin{array}{r}71 . \\
70 \\
93 . \\
104 . \\
44 .\end{array}$ & $\begin{array}{r}.24 \\
.24 \\
.30 \\
.35 \\
.19\end{array}$ & $\begin{array}{l}48 . \\
41 . \\
52 . \\
51 . \\
53 .\end{array}$ & $\begin{array}{l}60 . \\
65^{\circ} \\
49 . \\
51 . \\
49 .\end{array}$ \\
\hline
\end{tabular}

$\begin{array}{llllllll}76 . & 40 . & 0.00 & 64 . & .30 & 53 . & 48 . & 334 . \\ 77 . & 38 . & 0.00 & 60 . & .31 & 56 . & 37 . & 533 . \\ 76 . & 41 . & 0.00 & 63 . & .33 & 57 . & 45 . & 513 . \\ 72 . & 43 . & .06 & 64 . & .32 & 52 . & 68 . & 396 . \\ 68 . & 38 . & 0.00 & 67 . & .24 & 51 . & 56 . & 402 .\end{array}$

$\begin{array}{rrrrrrrr}72 . & 38 . & 0.00 & 99 . & .38 & 53 . & 52 . & 469 . \\ 64 . & 390^{\circ} & 0.00 & 72 . & .21 & 50 . & 66 . & 327 . \\ 62 . & 40 . & 0.00 & 147 . & .34 & 48 . & 53 . & 419 . \\ 60 . & 27 \circ & 0.00 & 176 . & .36 & 44 . & 34 . & 541 . \\ 57 . & 34 . & 0.00 & 95 . & .22 & 39 . & 55 . & 474 .\end{array}$

$\begin{array}{rrrrrrrr}66 . & 35 . & 0.00 & 81 . & .25 & 46 . & 68 . & 442 . \\ 70 . & 38 . & 0.00 & 81 . & .28 & 50 . & 53 . & 435 . \\ 68 . & 40 . & 0.00 & 148 . & .38 & 54 . & 45 . & 426 . \\ 63 . & 43 . & .02 & 77 . & .20 & 46 . & 66 . & 356 . \\ 57 . & 37 . & 0.00 & 170 . & .28 & 42 . & 48 . & 359 .\end{array}$

$\begin{array}{llllllll}50 . & 35 . & .17 & 61 . & .00 & 29 . & 90 . & 88 . \\ 47 . & 33 . & .11 & 54 . & .00 & 28 . & 86 . & 314 . \\ 55 . & 31 . & 0.00 & 58 . & .13 & 31 . & 54 . & 484 . \\ 65 . & 34 . & 0.00 & 58 . & .18 & 41 . & 56 . & 438 . \\ 68 . & 37 . & 0.00 & 53 . & .19 & 47 . & 47 . & 432 . \\ 66 . & 36 . & 0.00 & 82 . & .21 & 42 . & 67 . & 276 . \\ 58 . & 39 . & 0.00 & 42 . & .12 & 43 . & 73 . & 274 . \\ 67 . & 37 . & 0.00 & 74 . & .12 & 45 . & 49 . & 435 . \\ 64 . & 37 . & 0.00 & 74 . & .35 & 45 . & 47 . & 311 . \\ 61 . & 38 . & 0.00 & 68 . & .21 & 47 . & 47 . & 300 \\ & & & & & & & \\ 66 . & 37 . & 0.00 & 90 . & .27 & 47 . & 44 . & 413 . \\ 64 . & 36 . & 0.00 & 92 . & .28 & 42 . & 47 . & 426 .\end{array}$


Table 5.--Climatic data, Fairplay station,

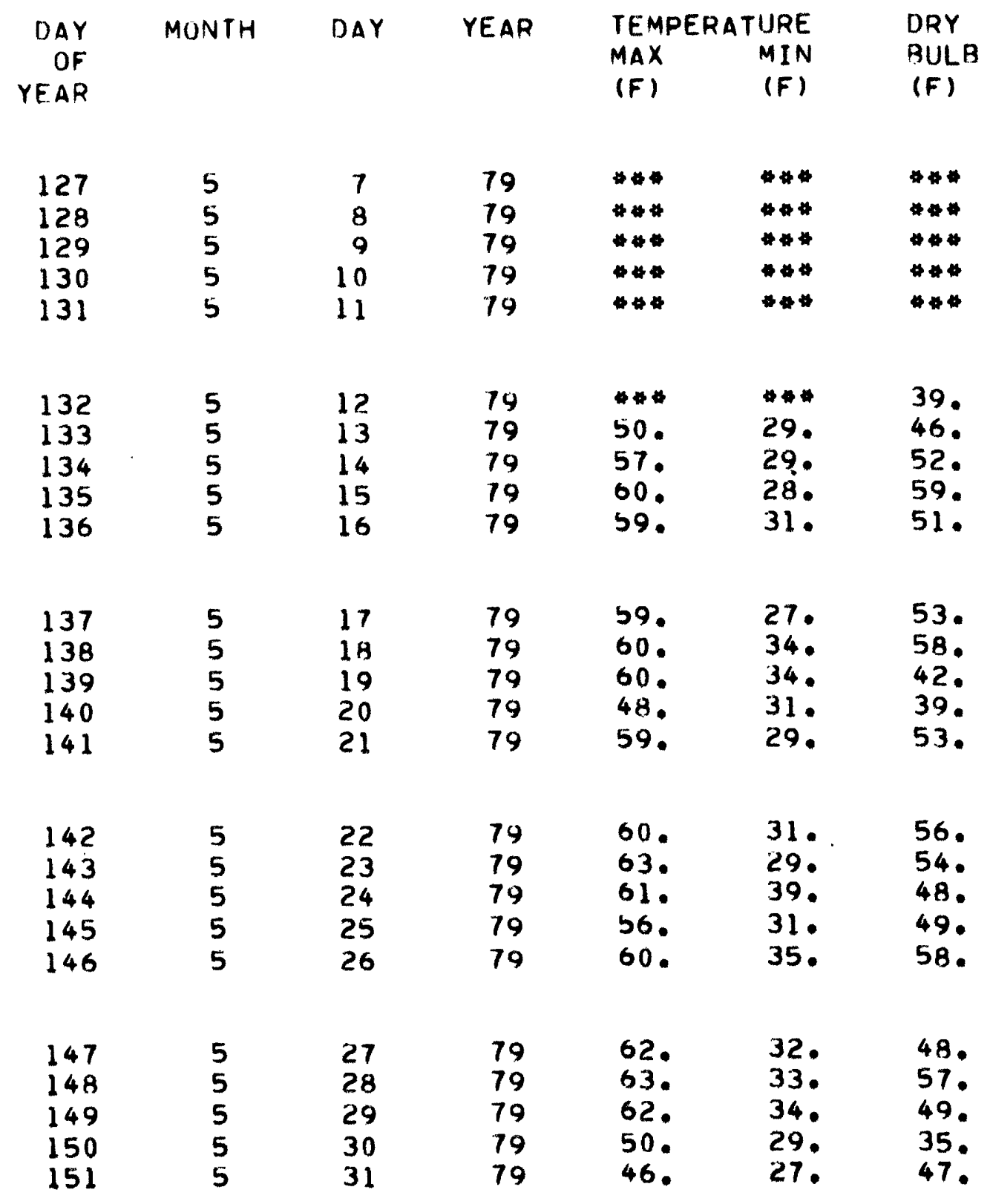


May-September 1977, 1978, and 1979--Continued

\begin{tabular}{|c|c|c|c|c|c|c|}
\hline $\begin{array}{l}\text { WET } \\
\text { BULB } \\
(F)\end{array}$ & $\begin{array}{l}\text { PAN } \\
\text { MAX } \\
\text { (F) }\end{array}$ & $\begin{array}{r}\text { WATER } \\
\text { MIN } \\
\text { (F) }\end{array}$ & $\begin{array}{l}\text { PRECIP } \\
\text { (IN.) }\end{array}$ & $\begin{array}{l}\text { WIND } \\
\text { (MI./DAY) }\end{array}$ & $\begin{array}{l}\text { PAN } \\
\text { EVAP } \\
(I N .)\end{array}$ & $\begin{array}{l}\text { SOLAR } \\
\text { RAD } \\
(L Y .)\end{array}$ \\
\hline
\end{tabular}

\begin{tabular}{|c|c|c|c|c|c|}
\hline $4 * *$ & 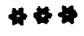 & 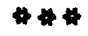 & 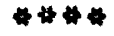 & $\forall * * *$ & $\not \Delta$ \\
\hline $4 * 4$ & * & $4 * 4$ & 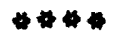 & $\forall * * *$ & 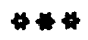 \\
\hline 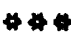 & $4+4$ & 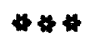 & 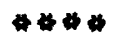 & $4 * 4 * 4$ & $4 \Delta$ \\
\hline $4 * *$ & 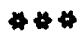 & $\omega *$ & $4 * * *$ & $\forall * 4 *$ & $4 * 4$ \\
\hline 444 & *⿻一从十 $* *$ & $4 * 4$ & 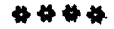 & $4 * 4 *$ & 4 \\
\hline
\end{tabular}

\begin{tabular}{|c|c|c|c|c|c|}
\hline $\begin{array}{l}33 . \\
37 \circ^{\circ} \\
41 . \\
43 . \\
43 .\end{array}$ & 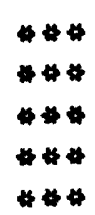 & 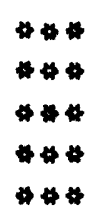 & $\begin{array}{l}0.00 \\
0.00 \\
0.00 \\
0.00 \\
0.00\end{array}$ & 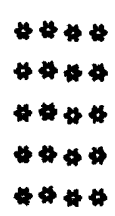 & 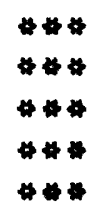 \\
\hline
\end{tabular}

\begin{tabular}{|c|c|c|c|c|c|}
\hline $\begin{array}{l}41 . \\
44{ }^{\circ} \\
40 \\
38 \\
40\end{array}$ & 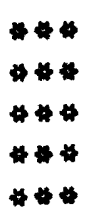 & 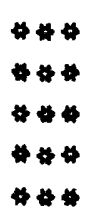 & $\begin{array}{r}0.00 \\
0.00 \\
.12 \\
.01 \\
0.00\end{array}$ & 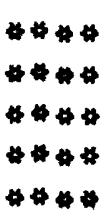 & 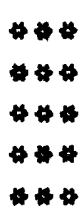 \\
\hline
\end{tabular}

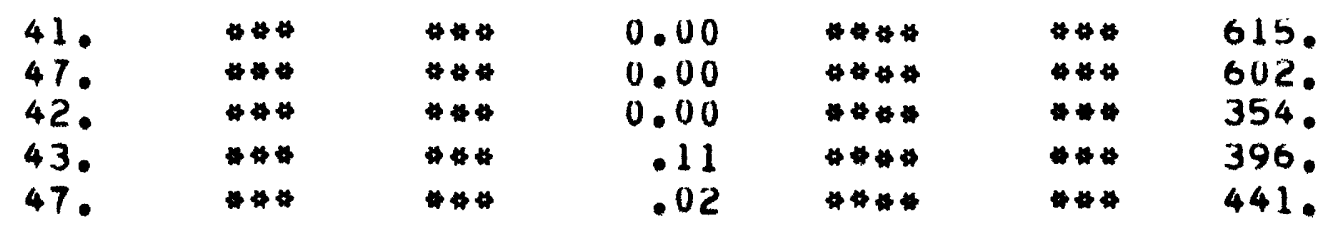

\begin{tabular}{|c|c|c|c|c|c|}
\hline $\begin{array}{l}42 . \\
44 \\
40 \\
35 \\
36\end{array}$ & 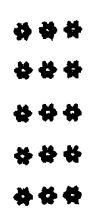 & 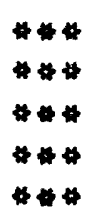 & $\begin{array}{r}.01 \\
0.00 \\
0.00 \\
.36 \\
.21\end{array}$ & 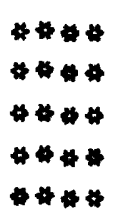 & 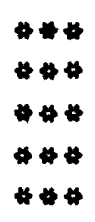 \\
\hline
\end{tabular}


Table 5.--Climatic data, Faimplay station,

\begin{tabular}{|c|c|c|c|c|c|c|}
\hline $\begin{array}{r}\text { DAY } \\
\text { OF } \\
Y E A R\end{array}$ & MONTH & DAY & YEAR & $\begin{array}{l}\text { TEMP } \\
\text { MAX } \\
\text { (F) }\end{array}$ & $\begin{array}{l}\text { TURE } \\
\text { MIN } \\
\text { (F) }\end{array}$ & $\begin{array}{l}\text { DRY } \\
\text { BULR } \\
\text { (F) }\end{array}$ \\
\hline $\begin{array}{l}152 \\
153 \\
154 \\
155 \\
156\end{array}$ & $\begin{array}{l}6 \\
6 \\
6 \\
6 \\
6\end{array}$ & $\begin{array}{l}1 \\
2 \\
3 \\
4 \\
5\end{array}$ & $\begin{array}{l}79 \\
79 \\
79 \\
79 \\
79\end{array}$ & $\begin{array}{l}48 . \\
57 . \\
60 . \\
62 . \\
66 .\end{array}$ & $\begin{array}{l}250^{\circ} \\
320^{\circ} \\
29{ }^{\circ} \\
33{ }^{\circ}\end{array}$ & $\begin{array}{l}40 . \\
53 . \\
60 \\
57 \\
64 .\end{array}$ \\
\hline $\begin{array}{l}157 \\
158 \\
159 \\
160 \\
161\end{array}$ & $\begin{array}{l}6 \\
6 \\
6 \\
6 \\
6\end{array}$ & $\begin{array}{r}6 \\
7 \\
8 \\
9 \\
10\end{array}$ & $\begin{array}{l}79 \\
79 \\
79 \\
79 \\
79\end{array}$ & $\begin{array}{l}68 . \\
650^{\circ} \\
450^{\circ} \\
390^{\circ}\end{array}$ & $\begin{array}{l}35 . \\
350^{\circ} \\
31 . \\
28 . \\
21 .\end{array}$ & $\begin{array}{l}64 . \\
43 . \\
33 . \\
36 . \\
49 .\end{array}$ \\
\hline $\begin{array}{l}162 \\
163 \\
164 \\
165 \\
166\end{array}$ & $\begin{array}{l}6 \\
6 \\
6 \\
6 \\
6\end{array}$ & $\begin{array}{l}11 \\
12 \\
13 \\
14 \\
15\end{array}$ & $\begin{array}{l}79 \\
79 \\
79 \\
79 \\
79\end{array}$ & $\begin{array}{l}63 . \\
70 \\
70 \\
70\end{array}$ & $\begin{array}{l}28 . \\
36 \\
36 \\
36\end{array}$ & $\begin{array}{l}61 . \\
65 \\
4 * * \\
63 .\end{array}$ \\
\hline $\begin{array}{l}161 \\
168 \\
169 \\
170 \\
171\end{array}$ & $\begin{array}{l}6 \\
6 \\
6 \\
6 \\
6\end{array}$ & $\begin{array}{l}16 \\
17 \\
18 \\
19 \\
20\end{array}$ & $\begin{array}{l}79 \\
79 \\
79 \\
79 \\
79\end{array}$ & $\begin{array}{l}69 . \\
67 . \\
60 . \\
51 . \\
64 .\end{array}$ & $\begin{array}{l}29 . \\
30 . \\
30 \\
23 . \\
38 .\end{array}$ & $\begin{array}{l}65 . \\
37 \\
50 \\
43 \\
62 .\end{array}$ \\
\hline $\begin{array}{l}172 \\
173 \\
174 \\
175 \\
176\end{array}$ & $\begin{array}{l}6 \\
6 \\
6 \\
6 \\
6\end{array}$ & $\begin{array}{l}21 \\
22 \\
23 \\
24 \\
25\end{array}$ & $\begin{array}{l}79 \\
79 \\
79 \\
79 \\
79\end{array}$ & $\begin{array}{l}69 . \\
71 . \\
68 . \\
69 . \\
70 .\end{array}$ & $\begin{array}{l}270^{\circ} \\
330^{\circ} \\
32 \\
430^{\circ}\end{array}$ & $\begin{array}{l}68 . \\
59 . \\
55 . \\
59 . \\
66 .\end{array}$ \\
\hline
\end{tabular}


May-September 1977, 1978, and 1979--Continued

\begin{tabular}{|c|c|c|c|c|c|c|}
\hline $\begin{array}{l}\text { WET } \\
\text { BULB } \\
\text { (F) }\end{array}$ & $\begin{array}{l}\text { PAN } \\
\text { MAX } \\
(F)\end{array}$ & $\begin{array}{l}\text { WATER } \\
\text { MIN } \\
\text { (F) }\end{array}$ & $\begin{array}{l}\text { PRECIP } \\
(I N,)\end{array}$ & $\begin{array}{l}\text { WIND } \\
\text { (MI./DAY) }\end{array}$ & $\begin{array}{l}\text { PAN } \\
\text { EVAP } \\
\text { (IN.) }\end{array}$ & $\begin{array}{l}\text { SOLAR } \\
\text { RAD } \\
\left(L Y_{.}\right)\end{array}$ \\
\hline $\begin{array}{l}36 . \\
38^{\circ} \\
43{ }^{\circ} \\
43 . \\
44 .\end{array}$ & $\begin{array}{l}65 . \\
69 \\
70 \\
69 \\
74\end{array}$ & $\begin{array}{l}38 . \\
40 . \\
41 . \\
40 . \\
40 .\end{array}$ & $\begin{array}{r}.01 \\
.33 \\
0.00 \\
0.00 \\
0.00\end{array}$ & $\begin{array}{l}91 . \\
52 . \\
79 . \\
69 . \\
75 .\end{array}$ & $\begin{array}{l}.20 \\
.18 \\
.30 \\
.24 \\
.30\end{array}$ & $\begin{array}{l}472^{\circ} \\
691^{\circ} \\
734^{\circ} \\
4090^{\circ} \\
570^{\circ}\end{array}$ \\
\hline $\begin{array}{l}45 . \\
43 . \\
33 . \\
31 . \\
36 .\end{array}$ & $\begin{array}{l}74 . \\
69 \\
48 \\
54 \\
71\end{array}$ & $\begin{array}{l}41 . \\
42 . \\
36 . \\
37 \\
37 .\end{array}$ & $\begin{array}{r}0.00 \\
.52 \\
1.58 \\
.48 \\
0.00\end{array}$ & $\begin{array}{l}94 . \\
90 . \\
45 . \\
93 . \\
59 .\end{array}$ & $\begin{array}{l}.28 \\
.26 \\
.04 \\
.25 \\
.16\end{array}$ & $\begin{array}{l}644 . \\
2070^{\circ} \\
220{ }^{\circ} \\
575{ }^{\circ} \\
700^{\circ}\end{array}$ \\
\hline $\begin{array}{l}45 \\
47 \\
40 \\
45\end{array}$ & $\begin{array}{l}72 . \\
77 \\
75 \\
75\end{array}$ & $\begin{array}{l}39 . \\
43, \\
44 . \\
40\end{array}$ & $\begin{array}{l}0.00 \\
0.00 \\
* * * 4 \\
0.00 \\
* * * *\end{array}$ & $\begin{array}{r}73 . \\
65 . \\
4 * 4 \\
222 . \\
* 4 *\end{array}$ & $\begin{array}{l}.30 \\
.30 \\
.44 \\
.83 \\
.4\end{array}$ & $\begin{array}{l}732^{\circ} \\
740 . \\
7390^{\circ} \\
595^{\circ} \\
593^{\circ}\end{array}$ \\
\hline $\begin{array}{l}42 . \\
32 . \\
38 \\
36 \\
43 .\end{array}$ & $\begin{array}{l}71 . \\
71 . \\
64: \\
56 . \\
74 .\end{array}$ & $\begin{array}{l}40 . \\
39 . \\
39 . \\
34 . \\
43 .\end{array}$ & $\begin{array}{r}0.00 \\
0.00 \\
0.00 \\
.01 \\
0.00\end{array}$ & $\begin{array}{l}173{ }^{\circ} \\
1400^{\circ} \\
1360^{\circ} \\
168^{\circ} \\
1.30\end{array}$ & $\begin{array}{l}.63 \\
.48 \\
.36 \\
.21 \\
.25\end{array}$ & $\begin{array}{l}645 . \\
734 . \\
562 . \\
698 . \\
708 .\end{array}$ \\
\hline $\begin{array}{l}45 . \\
45 . \\
49 . \\
44 \\
48\end{array}$ & $\begin{array}{l}74 \\
72 \\
71 \\
75 \\
70\end{array}$ & $\begin{array}{l}40 . \\
40 . \\
42 . \\
49 . \\
42 .\end{array}$ & $\begin{array}{l}0.00 \\
0.00 \\
0.00 \\
0.00 \\
0.00\end{array}$ & $\begin{array}{r}87 . \\
107 . \\
64 . \\
90 . \\
60 .\end{array}$ & $\begin{array}{l}.36 \\
.42 \\
.26 \\
.35 \\
.28\end{array}$ & $\begin{array}{l}736 \\
622 \\
573 \\
683 \\
461\end{array}$ \\
\hline
\end{tabular}


Table 5.--Climatic data, Fairplay station,

\begin{tabular}{|c|c|c|c|c|c|c|}
\hline $\begin{array}{r}\text { DAY } \\
\text { OF } \\
\text { YEAR }\end{array}$ & MONTH & DAY & YEAR & $\begin{array}{l}\text { TEMP } \\
\text { MAX } \\
\text { (F) }\end{array}$ & $\begin{array}{l}\text { TURE } \\
\text { MIN } \\
\text { (F) }\end{array}$ & $\begin{array}{l}\text { DRY } \\
\text { BULB } \\
\text { (F) }\end{array}$ \\
\hline $\begin{array}{l}177 \\
178 \\
179 \\
180 \\
181\end{array}$ & $\begin{array}{l}6 \\
6 \\
6 \\
6 \\
6\end{array}$ & $\begin{array}{l}26 \\
27 \\
28 \\
29 \\
30\end{array}$ & $\begin{array}{l}79 \\
79 \\
79 \\
79 \\
79\end{array}$ & $\begin{array}{l}73 . \\
73 \\
75 \\
77 \\
72 .\end{array}$ & $\begin{array}{l}36 . \\
360^{\circ} \\
370^{\circ} \\
370^{\circ}\end{array}$ & $\begin{array}{l}62 \\
71 \\
46 \\
68 \\
51\end{array}$ \\
\hline $\begin{array}{l}182 \\
183 \\
184 \\
185 \\
186\end{array}$ & $\begin{array}{l}7 \\
7 \\
7 \\
7 \\
7\end{array}$ & $\begin{array}{l}1 \\
2 \\
3 \\
4 \\
5\end{array}$ & $\begin{array}{l}79 \\
79 \\
79 \\
79 \\
79\end{array}$ & $\begin{array}{l}67 . \\
67 . \\
70 . \\
66 . \\
68 .\end{array}$ & $\begin{array}{l}46 . \\
36 \\
37 \\
35 \\
37\end{array}$ & $\begin{array}{l}47 \\
56 . \\
59 \\
64 \\
\end{array}$ \\
\hline $\begin{array}{l}187 \\
188 \\
189 \\
190 \\
191\end{array}$ & $\begin{array}{l}7 \\
7 \\
7 \\
7 \\
7\end{array}$ & $\begin{array}{r}6 \\
7 \\
8 \\
9 \\
10\end{array}$ & $\begin{array}{l}79 \\
79 \\
79 \\
79 \\
79\end{array}$ & $\begin{array}{l}68 . \\
4 \$ \\
15 . \\
11 . \\
77 .\end{array}$ & $\begin{array}{l}36 \\
\$ \$ \\
32 . \\
36 \\
35\end{array}$ & $\begin{array}{l}* * * \\
* * * \\
* * * \\
* * * \\
73 .\end{array}$ \\
\hline $\begin{array}{l}192 \\
193 \\
194 \\
195 \\
196\end{array}$ & $\begin{array}{l}7 \\
7 \\
7 \\
7 \\
7\end{array}$ & $\begin{array}{l}11 \\
12 \\
13 \\
14 \\
15\end{array}$ & $\begin{array}{l}79 \\
79 \\
79 \\
79 \\
79\end{array}$ & $\begin{array}{l}76 . \\
760^{\circ} \\
74{ }^{\circ} \\
77\end{array}$ & $\begin{array}{l}41 . \\
410^{\circ} \\
370^{\circ} \\
41 .\end{array}$ & $\begin{array}{l}70 . \\
62 . \\
69 . \\
76 . \\
59 .\end{array}$ \\
\hline $\begin{array}{l}197 \\
198 \\
199 \\
200 \\
201\end{array}$ & $\begin{array}{l}7 \\
7 \\
7 \\
7 \\
7\end{array}$ & $\begin{array}{l}16 \\
17 \\
18 \\
19 \\
20\end{array}$ & $\begin{array}{l}79 \\
79 \\
79 \\
79 \\
79\end{array}$ & $\begin{array}{l}71 . \\
71 . \\
66 . \\
66 . \\
69 .\end{array}$ & $\begin{array}{l}42 . \\
38 . \\
34 \\
33 \\
35\end{array}$ & $\begin{array}{l}62 . \\
56 . \\
52 . \\
52 . \\
53 .\end{array}$ \\
\hline
\end{tabular}


May-September 1977, 1978, and 1979--Continued

\begin{tabular}{|c|c|c|c|c|c|}
\hline $\begin{array}{l}\text { WET } \\
\text { BULB } \\
\text { (F) }\end{array}$ & $\begin{array}{l}\text { PAN } \\
\text { MAX } \\
\text { (F) }\end{array}$ & $\begin{array}{l}\text { WATER } \\
\text { MIN } \\
\text { (F) }\end{array}$ & $\begin{array}{l}\text { PRECIP } \\
(I N .)\end{array}$ & $\begin{array}{l}\text { WIND } \\
\text { (MI./DAY) }\end{array}$ & $\begin{array}{l}\text { PAN } \\
\text { EVAP } \\
(I N .)\end{array}$ \\
\hline $\begin{array}{l}46 . \\
48 . \\
38 \\
50 \\
50\end{array}$ & $\begin{array}{l}70 \\
74 \\
78 \\
79 \\
75 .\end{array}$ & $\begin{array}{l}41 . \\
42 . \\
43 . \\
43 . \\
50\end{array}$ & $\begin{array}{r}0.00 \\
0.00 \\
0.00 \\
0.00 \\
.05\end{array}$ & $\begin{array}{l}75 . \\
74 . \\
80 . \\
54 . \\
74 .\end{array}$ & $\begin{array}{r}.26 \\
.32 \\
.42 \\
.29 \\
.23\end{array}$ \\
\hline $\begin{array}{l}36 . \\
* * 4 \\
* 4 \\
* 4 \\
* 4\end{array}$ & $\begin{array}{l}65 . \\
70 \\
71 . \\
74 \\
73^{\circ}\end{array}$ & $\begin{array}{l}47 \\
42 . \\
44 \\
44 \\
44\end{array}$ & $\begin{array}{r}0.00 \\
.01 \\
.03 \\
.03 \\
.15\end{array}$ & $\begin{array}{l}95 . \\
63 . \\
65 . \\
66 . \\
54 .\end{array}$ & $\begin{array}{r}.05 \\
.19 \\
.26 \\
.26 \\
.27\end{array}$ \\
\hline
\end{tabular}

\begin{tabular}{|c|c|c|c|c|c|}
\hline *4 & 66. & 45. & $\begin{array}{l}0.00 \\
\square \# \%\end{array}$ & 61. & $\begin{array}{r}.19 \\
.25\end{array}$ \\
\hline $\begin{array}{l}* 4 \% \\
46 .\end{array}$ & $\begin{array}{l}76 . \\
75 . \\
78 .\end{array}$ & $\begin{array}{l}40 . \\
41 . \\
41 .\end{array}$ & $\begin{array}{l}0.00 \\
0.00 \\
0.00\end{array}$ & $\begin{array}{l}56 . \\
88 . \\
62 .\end{array}$ & $\begin{array}{r}.25 \\
.40 \\
.36\end{array}$ \\
\hline
\end{tabular}

$\begin{array}{rrrrrrr}45 . & 80 . & 41 . & 0.00 & 76 . & .26 & 530 . \\ 48 . & 76 . & 44 . & .01 & 56 . & .28 & 383 . \\ 47 . & 77 . & 42 . & 0.00 & 90 . & .32 & 711 . \\ 50 . & 80 . & 42 . & 0.00 & 71 . & .67 & 697 . \\ 49 . & 73 . & 47 . & .30 & 60 . & .31 & 382 . \\ & & & & & & \\ 46 . & 70 . & 49 . & .01 & 82 . & .32 & 367 . \\ 48 . & 67 . & 44 . & .08 & 66 . & .15 & 433 . \\ 46 . & 65 . & 43 . & .55 & 63 . & .47 & 392 . \\ 45 . & 73 . & 40 . & .01 & 57 . & .22 & 596 . \\ 45 . & 72 . & 45 . & 0.00 & 47 . & .21 & 458 .\end{array}$


Table 5.--Climatic data, Faimplay station,

\begin{tabular}{|c|c|c|c|c|c|c|}
\hline $\begin{array}{r}\text { DAY } \\
\text { OF } \\
\text { YEAR }\end{array}$ & MONTH & DAY & YEAR & $\begin{array}{l}\text { TEMP } \\
\text { MAX } \\
\text { (F) }\end{array}$ & $\begin{array}{l}\text { TURE } \\
\text { MIN } \\
\text { (F) }\end{array}$ & $\begin{array}{l}\text { DRY } \\
\text { BULB } \\
(F)\end{array}$ \\
\hline $\begin{array}{l}202 \\
203 \\
204 \\
205 \\
206\end{array}$ & $\begin{array}{l}7 \\
7 \\
7 \\
7 \\
7\end{array}$ & $\begin{array}{l}21 \\
22 \\
23 \\
24 \\
25\end{array}$ & $\begin{array}{l}79 \\
79 \\
79 \\
79 \\
79\end{array}$ & $\begin{array}{l}69 . \\
4:{ }^{\circ} \\
73 . \\
71 . \\
75 .\end{array}$ & $\begin{array}{l}39 . \\
36 . \\
43 . \\
40 .\end{array}$ & $\begin{array}{l}49 . \\
61 . \\
70 \\
70\end{array}$ \\
\hline $\begin{array}{l}207 \\
208 \\
209 \\
210 \\
211\end{array}$ & $\begin{array}{l}7 \\
7 \\
7 \\
7 \\
7\end{array}$ & $\begin{array}{l}26 \\
27 \\
28 \\
29 \\
30\end{array}$ & $\begin{array}{l}79 \\
79 \\
79 \\
79 \\
79\end{array}$ & $\begin{array}{l}76 . \\
72^{\circ} \\
76 . \\
74 . \\
74 .\end{array}$ & $\begin{array}{l}37 . \\
40 . \\
38 . \\
40 . \\
39 .\end{array}$ & $\begin{array}{l}60 . \\
54 . \\
74 . \\
68^{\circ} \\
64 .\end{array}$ \\
\hline $\begin{array}{l}212 \\
213 \\
214 \\
215 \\
216\end{array}$ & $\begin{array}{l}7 \\
8 \\
8 \\
8 \\
8\end{array}$ & $\begin{array}{r}31 \\
1 \\
2 \\
3 \\
4\end{array}$ & $\begin{array}{l}79 \\
79 \\
79 \\
79 \\
79\end{array}$ & $\begin{array}{l}69 . \\
74{ }^{\circ} \\
73 . \\
77 . \\
80 .\end{array}$ & $\begin{array}{l}38 \\
31 . \\
34 . \\
37 \\
38\end{array}$ & $\begin{array}{l}48 . \\
500^{\circ} \\
50 \\
610^{\circ} \\
45 .\end{array}$ \\
\hline $\begin{array}{l}217 \\
218 \\
219 \\
220 \\
221\end{array}$ & $\begin{array}{l}8 \\
8 \\
8 \\
8 \\
8\end{array}$ & $\begin{array}{l}5 \\
6 \\
7 \\
8 \\
9\end{array}$ & $\begin{array}{l}79 \\
79 \\
79 \\
79 \\
79\end{array}$ & $\begin{array}{l}78 . \\
80 . \\
79 . \\
77 . \\
69 .\end{array}$ & $\begin{array}{l}38 . \\
38 \\
45 . \\
48 \\
46 .\end{array}$ & $\begin{array}{l}63 . \\
60 . \\
71 . \\
48 . \\
57 .\end{array}$ \\
\hline $\begin{array}{l}222 \\
223 \\
224 \\
225 \\
226\end{array}$ & $\begin{array}{l}8 \\
8 \\
8 \\
8 \\
8\end{array}$ & $\begin{array}{l}10 \\
11 \\
12 \\
13 \\
14\end{array}$ & $\begin{array}{l}79 \\
79 \\
79 \\
79 \\
79\end{array}$ & $\begin{array}{l}60 . \\
12{ }^{\circ} \\
73{ }^{\circ} \\
67{ }^{\circ} \\
64 .\end{array}$ & $\begin{array}{l}390^{\circ} \\
40 . \\
390^{\circ} \\
40 . \\
46 .\end{array}$ & $\begin{array}{l}53 . \\
70 \\
66 . \\
64 . \\
52 .\end{array}$ \\
\hline
\end{tabular}


May-September 1977, 1978, and 1979--Continued

\begin{tabular}{|c|c|c|c|c|c|}
\hline $\begin{array}{l}\text { WET } \\
\text { BULG } \\
\text { (F) }\end{array}$ & $\begin{array}{l}\text { PAN } \\
\text { MAX } \\
\text { (F) }\end{array}$ & $\begin{array}{r}\text { WATER } \\
\text { MI } \\
\text { IF }\end{array}$ & $\begin{array}{l}\text { PRECIP } \\
\left(I N_{0}\right)\end{array}$ & $\begin{array}{l}\text { WIND } \\
\text { (MI./DAY) }\end{array}$ & $\begin{array}{l}\text { PAN } \\
\text { EVAP } \\
(I N,)\end{array}$ \\
\hline
\end{tabular}

$\begin{array}{lllllll}47 . & 65 . & 45 . & .16 & 41 . & .15 & 348 . \\ 44 & 74 . * & 44 . & 0.00 & 70 . & .25 & 603 . \\ 47 . & 74 . & 49 . & 0.00 & 58 . & .25 & 459 . \\ 52 . & 79 . & 49 . & 567 . \\ 49 . & 80 . & 52 . & 0.00 & 60 . & .39 & 556 .\end{array}$

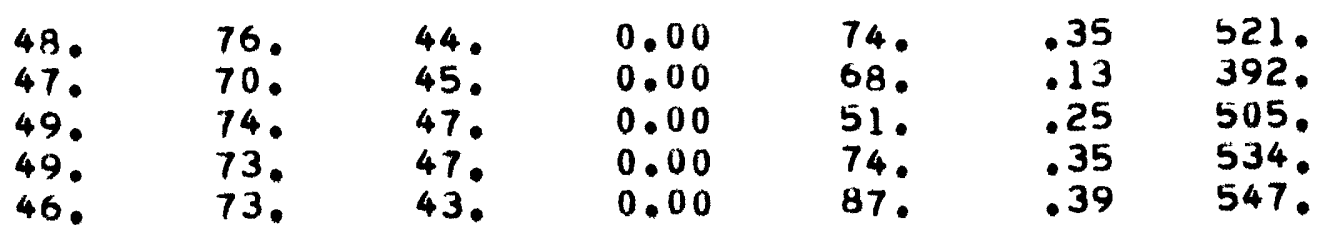

$\begin{array}{lllllll}48 . & 70 . & 47 . & 0.00 & 72 . & .42 & 361 . \\ 43 . & 77 . & 42 . & 0.00 & 67 . & .21 & 632 . \\ 46 . & 72 . & 41 . & 0.00 & 62 . & .30 & 484 . \\ 46 . & 80 . & 44 . & 0.00 & 59 . & .32 & 636 . \\ 42 . & 81 . & 42 . & 0.00 & 57 . & .40 & 592 .\end{array}$

$\begin{array}{rllllll}47 . & 82 . & 42 . & 0.00 & 59 . & .39 & 649 . \\ 44 . & 76 . & 42 . & 0.00 & 67 . & .46 & 374 . \\ 50 . & 80 . & 46 . & 0.00 & 54 . & .30 & 621 . \\ 47 . & 82 . & 50 . & 0.00 & 72 . & .39 & 393 . \\ 52 . & 65 . & 49 . & .20 & 55 . & .14 & 321 .\end{array}$

\begin{tabular}{|c|c|c|c|c|c|}
\hline $\begin{array}{l}50 \\
38 \\
4\end{array}$ & $\begin{array}{l}69 . \\
80 . \\
80 . \\
71 . \\
56 .\end{array}$ & $\begin{array}{l}49 . \\
50 . \\
47 \\
47 \\
50\end{array}$ & $\begin{array}{r}.03 \\
.01 \\
0.00 \\
.01 \\
.75\end{array}$ & $\begin{array}{l}45 . \\
44 . \\
58 . \\
60 \\
30\end{array}$ & $\begin{array}{l}11 \\
.19 \\
.31 \\
.16 \\
.01\end{array}$ \\
\hline
\end{tabular}


Table 5.--Climatic data, Fairplay station,

\begin{tabular}{|c|c|c|c|c|c|c|}
\hline $\begin{array}{l}\text { DAY } \\
\text { OF } \\
\text { YEAR }\end{array}$ & MONTH & DAY & YEAR & $\begin{array}{l}\text { TEMP } \\
\text { MAX } \\
\text { (F) }\end{array}$ & $\begin{array}{l}\text { TURE } \\
\text { MIN } \\
\text { (F) }\end{array}$ & $\begin{array}{l}\text { DRY } \\
\text { BULR } \\
\text { (F) }\end{array}$ \\
\hline $\begin{array}{l}227 \\
228 \\
229 \\
230 \\
231\end{array}$ & $\begin{array}{l}8 \\
8 \\
8 \\
8 \\
8\end{array}$ & $\begin{array}{l}15 \\
16 \\
17 \\
18 \\
19\end{array}$ & $\begin{array}{l}79 \\
79 \\
79 \\
79 \\
79\end{array}$ & $\begin{array}{l}57 . \\
62 . \\
62 . \\
59 . \\
59 .\end{array}$ & $\begin{array}{l}43 . \\
40 . \\
35 . \\
40 . \\
31 .\end{array}$ & $\begin{array}{l}56 . \\
56 . \\
47 \\
46 . \\
54 .\end{array}$ \\
\hline $\begin{array}{l}232 \\
233 \\
234 \\
235 \\
236\end{array}$ & $\begin{array}{l}8 \\
8 \\
8 \\
8 \\
8\end{array}$ & $\begin{array}{l}20 \\
21 \\
22 \\
23 \\
24\end{array}$ & $\begin{array}{l}79 \\
79 \\
79 \\
79 \\
79\end{array}$ & $\begin{array}{l}36 . \\
62 . \\
65 . \\
65 . \\
65 .\end{array}$ & $\begin{array}{l}31 . \\
28 . \\
32 . \\
33 \\
35 .\end{array}$ & $\begin{array}{l}52 . \\
61 . \\
59 . \\
63 . \\
59 .\end{array}$ \\
\hline $\begin{array}{l}237 \\
238 \\
239 \\
240 \\
241\end{array}$ & $\begin{array}{l}8 \\
8 \\
8 \\
8 \\
8\end{array}$ & $\begin{array}{l}25 \\
26 \\
27 \\
28 \\
29\end{array}$ & $\begin{array}{l}79 \\
79 \\
79 \\
79 \\
79\end{array}$ & $\begin{array}{l}63 . \\
63 . \\
07 . \\
10 . \\
72 .\end{array}$ & $\begin{array}{l}38 . \\
34 . \\
32 . \\
34 . \\
34 .\end{array}$ & $\begin{array}{l}56 . \\
54 . \\
63 . \\
63 . \\
52 .\end{array}$ \\
\hline $\begin{array}{l}242 \\
243 \\
244 \\
245 \\
246\end{array}$ & $\begin{array}{l}8 \\
8 \\
9 \\
9 \\
9\end{array}$ & $\begin{array}{r}30 \\
31 \\
1 \\
2 \\
3\end{array}$ & $\begin{array}{l}79 \\
79 \\
79 \\
79 \\
79\end{array}$ & $\begin{array}{l}65 . \\
68 \\
73 \\
69 \\
10\end{array}$ & $\begin{array}{l}36 . \\
32 . \\
29 . \\
30 . \\
35 .\end{array}$ & $\begin{array}{l}50 . \\
59 . \\
66 . \\
67 . \\
66 .\end{array}$ \\
\hline $\begin{array}{l}247 \\
248 \\
249 \\
250 \\
251\end{array}$ & $\begin{array}{l}9 \\
9 \\
9 \\
9 \\
9\end{array}$ & $\begin{array}{l}4 \\
5 \\
6 \\
7 \\
8\end{array}$ & $\begin{array}{l}79 \\
79 \\
79 \\
79 \\
79\end{array}$ & $\begin{array}{l}71 . \\
74 . \\
75 \circ \\
71 . \\
72 .\end{array}$ & $\begin{array}{l}34 . \\
33 . \\
36 . \\
38 . \\
37 .\end{array}$ & $\begin{array}{l}68 \\
63 \\
71 \\
67 \\
58\end{array}$ \\
\hline
\end{tabular}


May-September 1977, 1978, and 1979--Continued

\begin{tabular}{|c|c|c|c|c|c|}
\hline $\begin{array}{l}\text { WET } \\
\text { BULB } \\
(F)\end{array}$ & $\begin{array}{l}\text { PAN } \\
\text { MAX } \\
\text { (F) }\end{array}$ & $\begin{array}{l}\text { WATER } \\
\text { MIN } \\
\text { (F) }\end{array}$ & $\begin{array}{l}\text { PRECIP } \\
(I N \cdot)\end{array}$ & $\begin{array}{l}\text { WIND } \\
\text { (MI./DAY) }\end{array}$ & $\begin{array}{l}\text { PAN } \\
\text { EVAP } \\
(I N .)\end{array}$ \\
\hline
\end{tabular}

\begin{tabular}{|c|c|c|c|c|c|c|}
\hline 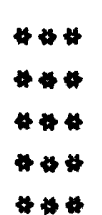 & $\begin{array}{l}61 . \\
68 . \\
66 . \\
61 . \\
64 .\end{array}$ & $\begin{array}{l}48 \\
48 \\
43 \\
46 \\
40\end{array}$ & $\begin{array}{r}.82 \\
.13 \\
.01 \\
.09 \\
0.00\end{array}$ & $\begin{array}{l}58 . \\
48 . \\
63 . \\
46 . \\
50 .\end{array}$ & $\begin{array}{r}.13 \\
.15 \\
.17 \\
.22 \\
.13\end{array}$ & $\begin{array}{l}340 . \\
367 . \\
397 . \\
249 . \\
504 .\end{array}$ \\
\hline
\end{tabular}

$\begin{array}{lllllll}* * & 63 . & 41 . & 0.00 & 87 . & .20 & 436 . \\ * * & 65 . & 42 . & 0.00 & 95 . & .23 & 507 . \\ 46 . & 69 . & 41 . & 0.00 & 53 . & .21 & 472 . \\ 47 . & 68 . & 42 . & 0.00 & 42 . & .20 & 436 . \\ 43 . & 66 . & 42 . & 0.00 & 59 . & .23 & 445 .\end{array}$

$\begin{array}{lllllll}43 . & 67 . & 47 . & 0.00 & 51 . & .20 & 382 . \\ 46 . & 65 . & 42 . & 0.00 & 48 . & .17 & 338 . \\ 44 . & 69 . & 40 . & 0.00 & 54 . & .20 & 387 . \\ 43 . & 69 . & 41 . & 0.00 & 71 . & .28 & 448 . \\ 41 . & 74 . & 40 . & 0.00 & 79 . & .33 & 426 .\end{array}$

$\begin{array}{lllllll}42 . & 61 . & 42 . & .01 & 59 . & .14 & 328 . \\ 41 . & 71 . & 40 . & 0.00 & 74 . & .27 & 553 . \\ 44 . & 73 . & 40 . & 0.00 & 63 . & .28 & 569 . \\ 45 . & 70 . & 41 . & 0.00 & 63 . & .28 & 432 . \\ 45 . & 69 . & 40 . & 0.00 & 60 . & .23 & 376 .\end{array}$

$\begin{array}{rrrrrrr}45 . & 67 . & 43 . & 0.00 & 42 . & .31 & 343 . \\ 45 . & 73 . & 40 . & 0.00 & 47 . & .16 & 433 . \\ 47 . & 75 . & 42 . & 0.00 & 48 . & .15 & 510 . \\ 46 . & 74 . & 48 . & .01 & 45 . & .25 & 298 . \\ 46 . & 64 . & 42 . & 0.00 & 45 . & .18 & 243 .\end{array}$


Table 5.--Climatic data, Faimplay station,

DAY MONTH
OF
YEAR
DAY YEAR

TEMPERATURE

MAX MIN

(F)

(F)

DRY

BULA

(F)

252

253

254

255

256

257

258

259

260

261

262

263

264

265

266

9
9
9
9
9

9
10
11
12
13

79

79

19

79

73.

37.

72.

38 .

68.

35 .

65.

30 .

57 .

35 .

62.

68.

55.

50 .

55 .

9
9
9
9
9

14

79

15

16

17

19

55.

31.

63.

69.

15.

65.

26.

79

61.

33.

28.

45.

59 .

61 .

18

79

$19 \quad 79$

20

21

22

2.3

66.

58.

62.

67.

27.

33.

32.

79
79

79

29.

32.

58.

54.

267

268

269

270

271

272

273

24
25
26
27
28

79

79

79

66.

64.

36.

31 .

30 .

b8.

28.

59.

27.

58.

40.

52.

48 .

59.

79

63.

66.

30.

30.

57.

79

64.

59. 
May-September 1977, 1978, and 1979--Continued

\begin{tabular}{|c|c|c|c|c|c|}
\hline $\begin{array}{l}\text { WET } \\
\text { BULB } \\
\text { (F) }\end{array}$ & $\begin{array}{l}\text { PAN } \\
\text { MAX } \\
\text { (F) }\end{array}$ & $\begin{array}{l}\text { WATER } \\
\text { MIN } \\
\text { (F) }\end{array}$ & $\begin{array}{l}\text { PRECIP } \\
(I N \cdot)\end{array}$ & $\begin{array}{l}\text { WIND } \\
(M I . / D \Delta Y)\end{array}$ & $\begin{array}{l}\text { PAN } \\
\text { EVAP } \\
\text { (IN.) }\end{array}$ \\
\hline $\begin{array}{l}47 . \\
48 \\
48 \\
42 \\
38\end{array}$ & $\begin{array}{l}69 . \\
73 . \\
68 . \\
68 . \\
63 .\end{array}$ & $\begin{array}{l}42 . \\
46 . \\
43 . \\
40 . \\
42 .\end{array}$ & $\begin{array}{l}0.00 \\
0.00 \\
0.00 \\
0.00 \\
0.00\end{array}$ & $\begin{array}{l}48 \\
73 . \\
65 \\
72 . \\
68\end{array}$ & $\begin{array}{l}.20 \\
.29 \\
.24 \\
.25 \\
.21\end{array}$ \\
\hline
\end{tabular}

$\begin{array}{lllllll}36 . & 56 . & 39 . & .10 & 62 . & .10 & 359 . \\ 38 . & 65 . & 37 . & 0.00 & 43 . & .16 & 488 . \\ 40 . & 69 . & 39 . & 0.00 & 49 . & .26 & 465 . \\ 38 . & 67 . & 39 . & 0.00 & 77 . & .30 & 461 . \\ 40 . & 67 . & 39 . & 0.00 & 72 . & .27 & 455 .\end{array}$

\begin{tabular}{|c|c|c|c|c|c|}
\hline $\begin{array}{l}41 . \\
37 . \\
41 . \\
41 . \\
42 .\end{array}$ & $\begin{array}{l}63 . \\
57 . \\
62 . \\
62 . \\
65 .\end{array}$ & $\begin{array}{l}39 . \\
40 \circ \\
40 \circ \\
40 \circ \\
40 .\end{array}$ & $\begin{array}{r}0.00 \\
.60 \\
.35 \\
0.00 \\
0.00\end{array}$ & $\begin{array}{l}71 . \\
56 . \\
35 . \\
60 . \\
58 .\end{array}$ & $\begin{array}{l}.23 \\
.13 \\
.10 \\
.18 \\
.18\end{array}$ \\
\hline $\begin{array}{l}42 . \\
430^{\circ} \\
41 . \\
37 . \\
40 .\end{array}$ & $\begin{array}{l}64 . \\
60 . \\
57 . \\
59 . \\
65 .\end{array}$ & $\begin{array}{l}40 . \\
40 . \\
39 . \\
40 . \\
37 .\end{array}$ & $\begin{array}{r}0.00 \\
.03 \\
0.00 \\
.01 \\
0.00\end{array}$ & $\begin{array}{l}83 . \\
48 . \\
58 . \\
77 . \\
71 .\end{array}$ & $\begin{array}{l}.23 \\
.12 \\
.13 \\
.14 \\
.19\end{array}$ \\
\hline 40. & $\begin{array}{l}69 . \\
66 .\end{array}$ & $\begin{array}{l}38 . \\
39 .\end{array}$ & $\begin{array}{l}0.00 \\
0.00\end{array}$ & $\begin{array}{l}61 . \\
63 .\end{array}$ & $\begin{array}{l}.21 \\
.23\end{array}$ \\
\hline
\end{tabular}




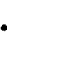


Table 6.--Climatic data, Antero Reservoir station, May-September 1977, 1978, and 1979

TEMPERATURE MAX: Maximum daily air temperature TEMPERATURE MIN: Minimum daily air temperature

PAN WATER MAX: Maximum daily water temperature of evaporation pan

PAN WATER MIN: Minimum daily water temperature of evaporation pan

PRECIP: Daily precipitation

PAN EVAP: Daily pan evaporation

DAILY AVG TEMP: Daily average air temperature DAILY AVG REL HUM: Daily average relative humidity

SOLAR RAD: Daily average solar radiation

F: Degrees Fahrenheit

IN: Inches

MI: Mile

LY: Langleys per minute

***: Represents missing data 
Table 6.--Climatic data, Antero Reservoir station,

\begin{tabular}{|c|c|c|c|c|c|c|c|}
\hline $\begin{array}{r}\text { DAY } \\
\text { OF } \\
\text { YEAR }\end{array}$ & MONTH & DAY & YEAR & $\begin{array}{l}\text { TEMF } \\
\text { MAX } \\
\text { (F) }\end{array}$ & $\begin{array}{l}\text { TURE } \\
\text { MIN } \\
\text { (F) }\end{array}$ & $\begin{array}{l}\text { DRY } \\
\text { BULA } \\
\text { (F) }\end{array}$ & $\begin{array}{l}\text { WET } \\
\text { BULB } \\
\text { (F) }\end{array}$ \\
\hline $\begin{array}{l}121 \\
122 \\
123 \\
124 \\
125\end{array}$ & $\begin{array}{l}5 \\
5 \\
5 \\
5 \\
5\end{array}$ & $\begin{array}{l}1 \\
2 \\
3 \\
4 \\
5\end{array}$ & $\begin{array}{l}77 \\
77 \\
77 \\
77 \\
77\end{array}$ & $\begin{array}{l}4.4 \\
62 . \\
63 . \\
62 . \\
24 .\end{array}$ & $\begin{array}{l}* 4 * \\
33 . \\
33 . \\
31 . \\
41 .\end{array}$ & $\begin{array}{l}* 4 \\
65 . \\
63 . \\
53 . \\
61 .\end{array}$ & $\begin{array}{l}44 \\
33 . \\
49 . \\
41 . \\
49 .\end{array}$ \\
\hline $\begin{array}{l}126 \\
127 \\
128 \\
129 \\
130\end{array}$ & $\begin{array}{l}5 \\
5 \\
5 \\
5 \\
5\end{array}$ & $\begin{array}{r}6 \\
7 \\
8 \\
9 \\
10\end{array}$ & $\begin{array}{l}77 \\
77 \\
77 \\
77 \\
77\end{array}$ & $\begin{array}{l}64 . \\
65 . \\
66 . \\
67 . \\
69 .\end{array}$ & $\begin{array}{l}43 . \\
38 . \\
24 . \\
26 . \\
31 .\end{array}$ & $\begin{array}{l}60 . \\
65 . \\
67 \\
69 \\
60\end{array}$ & $\begin{array}{l}45 . \\
51 . \\
55 . \\
58 . \\
50 .\end{array}$ \\
\hline $\begin{array}{l}131 \\
132 \\
133 \\
134 \\
135\end{array}$ & $\begin{array}{l}5 \\
5 \\
5 \\
5 \\
5\end{array}$ & $\begin{array}{l}11 \\
12 \\
13 \\
14 \\
15\end{array}$ & $\begin{array}{l}77 \\
77 \\
77 \\
77 \\
77\end{array}$ & $\begin{array}{l}62 . \\
61 . \\
62 . \\
65 . \\
54 .\end{array}$ & $\begin{array}{l}14 . \\
20 . \\
32 . \\
31 . \\
32 .\end{array}$ & $\begin{array}{l}60 . \\
62 . \\
62 . \\
40 . \\
54 .\end{array}$ & $\begin{array}{l}50 . \\
50 . \\
44 . \\
38 . \\
32 .\end{array}$ \\
\hline $\begin{array}{l}136 \\
137 \\
138 \\
139 \\
140\end{array}$ & $\begin{array}{l}5 \\
5 \\
5 \\
5 \\
5\end{array}$ & $\begin{array}{l}16 \\
17 \\
18 \\
19 \\
20\end{array}$ & $\begin{array}{l}77 \\
77 \\
77 \\
17 \\
77\end{array}$ & $\begin{array}{l}59 . \\
59 . \\
61 . \\
57 . \\
53 .\end{array}$ & $\begin{array}{l}25 . \\
30 . \\
28 . \\
29 . \\
30 .\end{array}$ & $\begin{array}{l}58 . \\
59 . \\
57 . \\
52 . \\
40 .\end{array}$ & $\begin{array}{l}47 . \\
450^{\circ} \\
41 . \\
40 \\
40\end{array}$ \\
\hline $\begin{array}{l}141 \\
142 \\
143 \\
144 \\
145\end{array}$ & $\begin{array}{l}5 \\
5 \\
5 \\
5 \\
5\end{array}$ & $\begin{array}{l}21 \\
22 \\
23 \\
24 \\
25\end{array}$ & $\begin{array}{l}77 \\
77 \\
77 \\
77 \\
77\end{array}$ & $\begin{array}{l}52 . \\
62 . \\
62 . \\
69 . \\
63 .\end{array}$ & $\begin{array}{l}17 . \\
17 \\
25 \\
27 \\
35 .\end{array}$ & $\begin{array}{l}50 . \\
61 . \\
62 . \\
62 . \\
46 .\end{array}$ & $\begin{array}{l}42 . \\
57 \\
58 \\
45 \\
40\end{array}$ \\
\hline
\end{tabular}


May-September 1977, 1978, and 1979--Continued

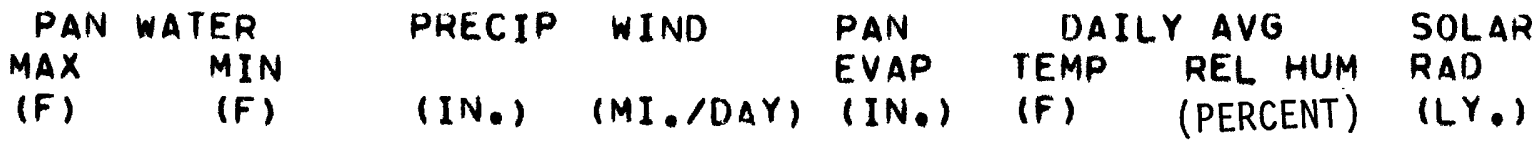

\begin{tabular}{|c|c|c|c|c|c|c|c|}
\hline $\begin{array}{l}6 \\
62 . \\
65 . \\
58 . \\
61 .\end{array}$ & $\begin{array}{l}* 4 \\
33 \\
35 \\
35 \\
44\end{array}$ & $\begin{array}{l}0.00 \\
0.00 \\
0.00 \\
0.00 \\
0.00\end{array}$ & $\begin{array}{r}44 \\
29 . \\
240 . \\
162 . \\
91 .\end{array}$ & $\begin{array}{r}.16 \\
.31 \\
.37 \\
.37 \\
.29\end{array}$ & 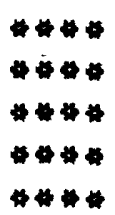 & 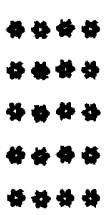 & 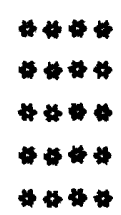 \\
\hline
\end{tabular}

\begin{tabular}{|c|c|c|c|c|c|c|c|}
\hline $\begin{array}{l}66 . \\
66 . \\
68 . \\
68 . \\
64 .\end{array}$ & $\begin{array}{l}35 \\
34 \\
35 \\
35 \\
35\end{array}$ & $\begin{array}{l}0.00 \\
0.00 \\
0.00 \\
0.00 \\
0.00\end{array}$ & $\begin{array}{r}92 . \\
85 . \\
76 \\
120 \\
114^{\circ}\end{array}$ & $\begin{array}{r}.36 \\
.33 \\
.34 \\
.26 \\
.34\end{array}$ & 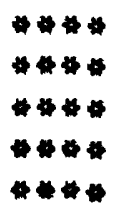 & 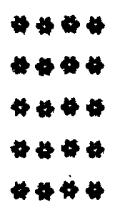 & 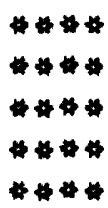 \\
\hline
\end{tabular}

\begin{tabular}{|c|c|c|c|c|c|c|c|}
\hline $\begin{array}{l}51 . \\
62 . \\
67 . \\
65 . \\
54 .\end{array}$ & $\begin{array}{l}31 . \\
350^{\circ} \\
35 . \\
35 \\
32\end{array}$ & $\begin{array}{r}0.00 \\
0.00 \\
0.00 \\
.22 \\
0.00\end{array}$ & $\begin{array}{l}77 . \\
121 . \\
102 . \\
214 . \\
105 .\end{array}$ & $\begin{array}{r}.17 \\
.39 \\
.08 \\
.28 \\
.22\end{array}$ & 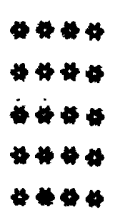 & 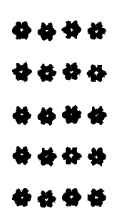 & 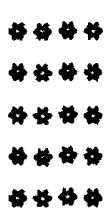 \\
\hline
\end{tabular}

\begin{tabular}{|c|c|c|c|c|c|c|c|}
\hline $\begin{array}{l}62 . \\
64 . \\
61 . \\
59 . \\
57 .\end{array}$ & $\begin{array}{l}31 . \\
33 . \\
34{ }^{\circ} \\
32 . \\
34 .\end{array}$ & $\begin{array}{r}0.00 \\
0.00 \\
0.00 \\
0.00 \\
.01\end{array}$ & $\begin{array}{r}199^{\circ} \\
186^{\circ} \\
144^{\circ} \\
73^{\circ} \\
97^{\circ}\end{array}$ & $\begin{array}{l}.49 \\
.33 \\
.33 \\
.15 \\
.22\end{array}$ & 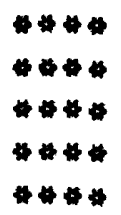 & 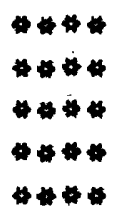 & 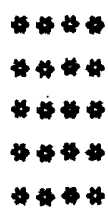 \\
\hline
\end{tabular}

\begin{tabular}{|c|c|c|c|c|c|c|c|}
\hline $\begin{array}{l}55 . \\
66 . \\
67 . \\
66 . \\
53 .\end{array}$ & $\begin{array}{l}31 . \\
32 \\
34 \\
35 . \\
35\end{array}$ & $\begin{array}{l}0.00 \\
0.00 \\
0.00 \\
0.00 \\
0.00\end{array}$ & $\begin{array}{l}74 . \\
111 . \\
105 . \\
101 . \\
114 .\end{array}$ & $\begin{array}{r}.25 \\
.42 \\
.29 \\
.13 \\
.30\end{array}$ & 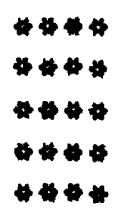 & 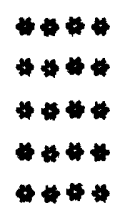 & $\begin{array}{l}* 4 * \\
4 * 4 \\
* * 4 \\
190 \\
527 .\end{array}$ \\
\hline
\end{tabular}


Table 6.--Climatic data, Antero Reservoir station,

\begin{tabular}{|c|c|c|c|c|c|c|c|}
\hline $\begin{array}{r}\text { OAY } \\
\text { OF } \\
\text { YEAR }\end{array}$ & MONTH & DAY & YEAR & $\begin{array}{l}\text { TEMF } \\
\text { MAX } \\
\text { (F) }\end{array}$ & $\begin{array}{l}\text { TURE } \\
\text { MIN } \\
\text { (F) }\end{array}$ & $\begin{array}{l}\text { DRY } \\
\text { BULA } \\
\text { (F) }\end{array}$ & $\begin{array}{l}\text { WET } \\
\text { BULB } \\
\text { (F) }\end{array}$ \\
\hline $\begin{array}{l}146 \\
147 \\
148 \\
149 \\
150\end{array}$ & $\begin{array}{l}5 \\
5 \\
5 \\
5 \\
5\end{array}$ & $\begin{array}{l}26 \\
27 \\
28 \\
29 \\
30\end{array}$ & $\begin{array}{l}77 \\
77 \\
17 \\
77 \\
77\end{array}$ & $\begin{array}{l}61 . \\
58 . \\
58 . \\
60 . \\
65 .\end{array}$ & $\begin{array}{l}22 . \\
21 . \\
21 . \\
25 . \\
25 .\end{array}$ & $\begin{array}{l}42 . \\
38 \\
40 \\
36 \\
36\end{array}$ & $\begin{array}{l}38 \\
35 \\
35 \\
36 \\
36\end{array}$ \\
\hline $\begin{array}{l}151 \\
152 \\
153 \\
154 \\
155\end{array}$ & $\begin{array}{l}5 \\
6 \\
6 \\
6 \\
6\end{array}$ & $\begin{array}{r}31 \\
1 \\
2 \\
3 \\
4\end{array}$ & $\begin{array}{l}77 \\
77 \\
77 \\
77 \\
77\end{array}$ & $\begin{array}{l}10 . \\
75 . \\
75 \circ \\
74 . \\
75 .\end{array}$ & $\begin{array}{l}35 . \\
33 . \\
32 . \\
28 . \\
30 .\end{array}$ & $\begin{array}{l}47 . \\
46 . \\
47 . \\
60 \\
49 .\end{array}$ & $\begin{array}{l}44 \\
44 \\
41 \\
48 \\
45\end{array}$ \\
\hline $\begin{array}{l}156 \\
157 \\
158 \\
159 \\
160\end{array}$ & $\begin{array}{l}6 \\
6 \\
6 \\
6 \\
6\end{array}$ & $\begin{array}{l}5 \\
6 \\
7 \\
8 \\
9\end{array}$ & $\begin{array}{l}77 \\
77 \\
77 \\
77 \\
77\end{array}$ & $\begin{array}{l}18 . \\
18 . \\
69 . \\
70 . \\
70 .\end{array}$ & $\begin{array}{l}29 . \\
41 . \\
40 . \\
37 . \\
39 .\end{array}$ & $\begin{array}{l}48 \\
47 \\
47 \\
49 \\
47\end{array}$ & $\begin{array}{l}450^{\circ} \\
470^{\circ} \\
470^{\circ} \\
47\end{array}$ \\
\hline $\begin{array}{l}161 \\
162 \\
163 \\
164 \\
165\end{array}$ & $\begin{array}{l}6 \\
6 \\
6 \\
6 \\
6\end{array}$ & $\begin{array}{l}10 \\
11 \\
12 \\
13 \\
14\end{array}$ & $\begin{array}{l}77 \\
77 \\
77 \\
77 \\
77\end{array}$ & $\begin{array}{l}68 . \\
10 . \\
69 . \\
12 . \\
16 .\end{array}$ & $\begin{array}{l}32 . \\
34 . \\
33 . \\
33 . \\
40 .\end{array}$ & $\begin{array}{l}46 . \\
53 \\
40 \\
41 . \\
55 .\end{array}$ & $\begin{array}{l}43 . \\
490^{\circ} \\
40 \\
40 \\
50\end{array}$ \\
\hline $\begin{array}{l}166 \\
167 \\
168 \\
169 \\
170\end{array}$ & $\begin{array}{l}6 \\
6 \\
6 \\
6 \\
6\end{array}$ & $\begin{array}{l}15 \\
16 \\
17 \\
18 \\
19\end{array}$ & $\begin{array}{l}77 \\
77 \\
77 \\
77 \\
17\end{array}$ & $\begin{array}{l}77 . \\
77 . \\
77 . \\
80 . \\
77 .\end{array}$ & $\begin{array}{l}31 . \\
32 . \\
34 . \\
45 . \\
33 .\end{array}$ & $\begin{array}{l}51 . \\
49 . \\
52 . \\
58 . \\
52 .\end{array}$ & $\begin{array}{l}44 \\
43 \\
44 \\
53 \\
49\end{array}$ \\
\hline
\end{tabular}


May-September 1977, 1978, and 1979--Continued

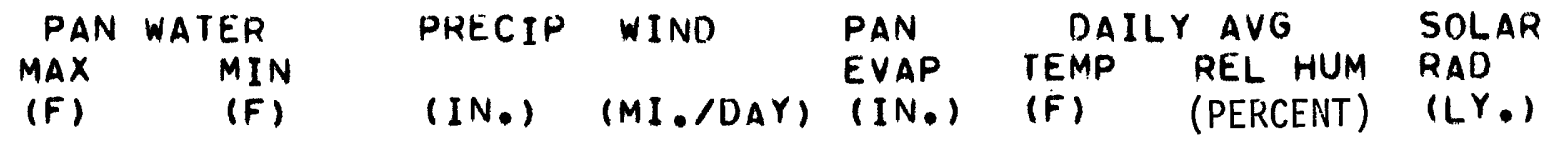

\begin{tabular}{|c|c|c|c|c|c|c|}
\hline $\begin{array}{l}63 . \\
57 \\
62 . \\
65 . \\
65\end{array}$ & $\begin{array}{l}33 \\
36 \\
31 . \\
35^{\circ} \\
34^{\circ}\end{array}$ & $\begin{array}{l}0.00 \\
0.00 \\
0.00 \\
0.00 \\
0.00\end{array}$ & $\begin{array}{r}95 . \\
106 \\
94 \\
85 \\
88\end{array}$ & $\begin{array}{r}.25 \\
.33 \\
.20 \\
.19 \\
.41\end{array}$ & 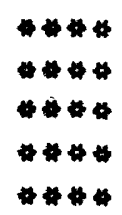 & 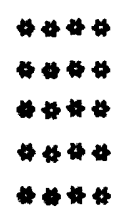 \\
\hline
\end{tabular}

\begin{tabular}{|c|c|c|c|c|c|c|}
\hline $\begin{array}{l}71 . \\
74 . \\
69 . \\
62 . \\
66 .\end{array}$ & $\begin{array}{l}35 \\
38 \\
40 \\
36 \\
38\end{array}$ & $\begin{array}{l}0.00 \\
0.00 \\
0.00 \\
0.00 \\
0.00\end{array}$ & $\begin{array}{l}50 . \\
66 . \\
67 . \\
62 . \\
73 .\end{array}$ & $\begin{array}{l}.32 \\
.40 \\
.22 \\
.26 \\
.42\end{array}$ & 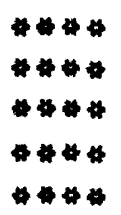 & 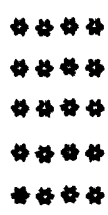 \\
\hline
\end{tabular}

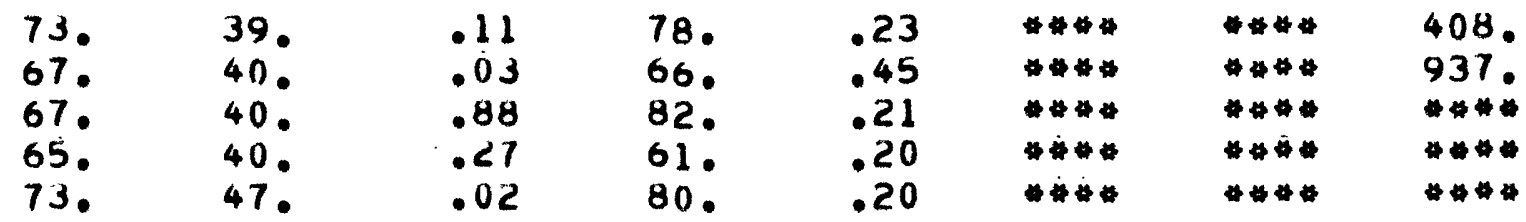

\begin{tabular}{|c|c|c|c|c|c|c|c|}
\hline $\begin{array}{l}66 . \\
69 . \\
61 . \\
73 . \\
75 .\end{array}$ & $\begin{array}{l}38 . \\
40 . \\
37 \circ^{\circ} \\
37 \circ^{\circ} \\
350^{\circ}\end{array}$ & $\begin{array}{r}0.00 \\
0.00 \\
.01 \\
0.00 \\
0.00\end{array}$ & $\begin{array}{l}69 . \\
76 . \\
67 . \\
82^{\circ} \\
57 .\end{array}$ & $\begin{array}{r}.30 \\
.24 \\
.36 \\
.36 \\
.41\end{array}$ & $\begin{array}{r}4 * 4 \\
4 * 4 \\
* 44 \\
4 * 4 \\
61 .\end{array}$ & 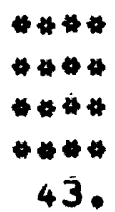 & $\begin{array}{l}4 * 4 \\
\# * 4 \\
4 * 4 \\
0 * 4 \\
632 .\end{array}$ \\
\hline
\end{tabular}

\begin{tabular}{|c|c|c|c|c|c|c|}
\hline $\begin{array}{l}79 . \\
71 . \\
72 . \\
74 . \\
75 .\end{array}$ & $\begin{array}{l}37 \\
38 \\
38 \\
38 \\
40\end{array}$ & $\begin{array}{l}0.00 \\
0.00 \\
0.00 \\
0.00 \\
0.00\end{array}$ & $\begin{array}{l}88 . \\
76 . \\
85 . \\
91 . \\
91 .\end{array}$ & $\begin{array}{r}.44 \\
.44 \\
.40 \\
.45 \\
.21\end{array}$ & $\begin{array}{l}59 . \\
60 . \\
60 . \\
63 . \\
58 .\end{array}$ & $\begin{array}{l}25 . \\
21 . \\
34 . \\
53 . \\
58 .\end{array}$ \\
\hline
\end{tabular}


Table 6.--Climatic data, Antero Reservoir station,

\begin{tabular}{|c|c|c|c|c|c|c|c|}
\hline $\begin{array}{r}\text { DAY } \\
\text { OF } \\
\text { YEAR }\end{array}$ & MONTH & DAY & YEAR & $\begin{array}{l}\text { TEMF } \\
\text { MAX } \\
\text { (F) }\end{array}$ & $\begin{array}{l}\text { TURE } \\
\text { MIN } \\
\text { (F) }\end{array}$ & $\begin{array}{l}\text { DRY } \\
\text { RULB } \\
\text { (F) }\end{array}$ & $\begin{array}{l}\text { WET } \\
\text { BULB } \\
\text { (F) }\end{array}$ \\
\hline $\begin{array}{l}171 \\
172 \\
173 \\
174 \\
175\end{array}$ & $\begin{array}{l}6 \\
6 \\
6 \\
6 \\
6\end{array}$ & $\begin{array}{l}20 \\
21 \\
22 \\
23 \\
24\end{array}$ & $\begin{array}{l}77 \\
77 \\
77 \\
77 \\
77\end{array}$ & $\begin{array}{l}76 . \\
11 . \\
12 . \\
75 . \\
10 .\end{array}$ & $\begin{array}{l}33 \\
33 \\
35 \\
45 \\
42\end{array}$ & $\begin{array}{l}47 . \\
50 . \\
450^{\circ} \\
50 . \\
52^{\circ}\end{array}$ & $\begin{array}{l}46 . \\
410^{\circ} \\
42 \\
48 \\
48\end{array}$ \\
\hline $\begin{array}{l}176 \\
177 \\
178 \\
179 \\
180\end{array}$ & $\begin{array}{l}6 \\
6 \\
6 \\
6 \\
6\end{array}$ & $\begin{array}{l}25 \\
26 \\
27 \\
28 \\
29\end{array}$ & $\begin{array}{l}77 \\
77 \\
77 \\
77 \\
77\end{array}$ & $\begin{array}{l}68 . \\
760^{\circ} \\
76 . \\
190^{\circ} \\
19 .\end{array}$ & $\begin{array}{l}34 . \\
40 \\
38 \\
39 \\
40\end{array}$ & $\begin{array}{l}49^{\circ} \\
44^{\circ} \\
55^{\circ} \\
540^{\circ} \\
50^{\circ}\end{array}$ & $\begin{array}{l}46 \\
44{ }^{\circ} \\
49^{\circ} \\
48 \\
48^{\circ}\end{array}$ \\
\hline $\begin{array}{l}181 \\
182 \\
183 \\
184 \\
185\end{array}$ & $\begin{array}{l}6 \\
7 \\
7 \\
7 \\
7\end{array}$ & $\begin{array}{r}30 \\
1 \\
2 \\
3 \\
4\end{array}$ & $\begin{array}{l}77 \\
77 \\
77 \\
77 \\
77\end{array}$ & $\begin{array}{l}17 . \\
80 . \\
79 . \\
77 . \\
81 .\end{array}$ & $\begin{array}{l}39 . \\
34{ }^{\circ} \\
44^{\circ} \\
38 \\
38^{\circ}\end{array}$ & $\begin{array}{l}49 . \\
460^{\circ} \\
62 . \\
53^{\circ} \\
58 .\end{array}$ & $\begin{array}{l}44 \\
43{ }^{\circ} \\
51 \\
48 \\
51\end{array}$ \\
\hline $\begin{array}{l}186 \\
187 \\
188 \\
189 \\
190\end{array}$ & $\begin{array}{l}7 \\
7 \\
7 \\
7 \\
7\end{array}$ & $\begin{array}{l}5 \\
6 \\
7 \\
8 \\
9\end{array}$ & $\begin{array}{l}77 \\
77 \\
77 \\
77 \\
77\end{array}$ & $\begin{array}{l}77 . \\
75 . \\
71 . \\
78 . \\
75 .\end{array}$ & $\begin{array}{l}41 . \\
36 . \\
33 . \\
41 . \\
34 .\end{array}$ & $\begin{array}{l}58 . \\
50 . \\
50 . \\
54 . \\
50 .\end{array}$ & $\begin{array}{l}53{ }^{\circ} \\
48 \\
46 . \\
49 . \\
44 .\end{array}$ \\
\hline $\begin{array}{l}191 \\
192 \\
193 \\
194 \\
195\end{array}$ & $\begin{array}{l}7 \\
7 \\
7 \\
7 \\
7\end{array}$ & $\begin{array}{l}10 \\
11 \\
12 \\
13 \\
14\end{array}$ & $\begin{array}{l}77 \\
77 \\
77 \\
77 \\
77\end{array}$ & $\begin{array}{l}14 . \\
75 . \\
74 . \\
79 . \\
72 .\end{array}$ & $\begin{array}{l}32 . \\
30 \\
34{ }^{\circ} \\
39 . \\
39 .\end{array}$ & $\begin{array}{l}49 . \\
42 . \\
51 . \\
61 . \\
53 .\end{array}$ & $\begin{array}{l}44{ }^{\circ} \\
39^{\circ} \\
43^{\circ} \\
55^{\circ} \\
49^{\circ}\end{array}$ \\
\hline
\end{tabular}


May-September 1977, 1978, and 1979--Continued

\begin{tabular}{|c|c|c|c|c|c|c|c|}
\hline $\begin{array}{l}\text { PAN } \\
\text { MAX } \\
\text { (F) }\end{array}$ & $\begin{array}{l}\text { WATER } \\
\text { MIN } \\
\text { (F) }\end{array}$ & $\begin{array}{l}\text { PRECIP } \\
\text { (IN.) }\end{array}$ & $\begin{array}{l}\text { WIND } \\
\text { (MI./DAY) }\end{array}$ & $\begin{array}{l}\text { PAN } \\
\text { EVAP } \\
\text { (IN,) }\end{array}$ & $\begin{array}{l}\text { DAI } \\
\text { TEMP } \\
\text { (F) }\end{array}$ & $\begin{array}{l}\text { Y AVG } \\
\text { REL HUM } \\
\text { (PERCENT) }\end{array}$ & $\begin{array}{l}\text { SOLAR } \\
\text { RAD } \\
\text { (LY.) }\end{array}$ \\
\hline $\begin{array}{l}72 . \\
70 \\
68 \\
74 \\
61\end{array}$ & $\begin{array}{l}41 . \\
36 \\
38 \\
39 \\
42\end{array}$ & $\begin{array}{r}0.00 \\
0.00 \\
0.00 \\
.06 \\
0.00\end{array}$ & $\begin{array}{r}138 . \\
72 \\
81 . \\
69 \\
54 .\end{array}$ & $\begin{array}{l}.53 \\
.36 \\
.33 \\
.16 \\
.24\end{array}$ & $\begin{array}{l}58 . \\
58 . \\
59 . \\
53 . \\
56 .\end{array}$ & $\begin{array}{l}45 . \\
29 . \\
34 . \\
75 \\
68 .\end{array}$ & $\begin{array}{l}590 . \\
580 . \\
624 . \\
363 . \\
521 .\end{array}$ \\
\hline $\begin{array}{l}67 . \\
73 . \\
70 . \\
77 . \\
76 .\end{array}$ & $\begin{array}{l}40 . \\
43{ }^{\circ} \\
41 \\
44{ }^{\circ} \\
44 .\end{array}$ & $\begin{array}{l}0.00 \\
.36 \\
0.00 \\
0.00 \\
0.00\end{array}$ & $\begin{array}{l}70 . \\
750^{\circ} \\
51 . \\
76 . \\
87 .\end{array}$ & $\begin{array}{l}.47 \\
.26 \\
.36 \\
.32 \\
.37\end{array}$ & $\begin{array}{l}57 . \\
59 . \\
64 . \\
61 . \\
62 .\end{array}$ & $\begin{array}{l}56 . \\
54 . \\
42 . \\
51 . \\
44 .\end{array}$ & $\begin{array}{l}632 . \\
586 . \\
613 . \\
612 . \\
516 .\end{array}$ \\
\hline $\begin{array}{l}68 . \\
76 . \\
71 . \\
71 . \\
74 .\end{array}$ & $\begin{array}{l}39 . \\
40 \\
41 . \\
430 \\
43 .\end{array}$ & $\begin{array}{l}0.00 \\
0.00 \\
0.00 \\
0.00 \\
0.00\end{array}$ & $\begin{array}{r}65 . \\
81 . \\
78 . \\
63 . \\
127 .\end{array}$ & $\begin{array}{l}.39 \\
.39 \\
.37 \\
.32 \\
.33\end{array}$ & $\begin{array}{l}61 . \\
62 . \\
64 . \\
64 . \\
63 .\end{array}$ & $\begin{array}{l}39 . \\
44 . \\
40 . \\
49 . \\
61 .\end{array}$ & $\begin{array}{l}732^{\circ} \\
613^{\circ} \\
5080^{\circ} \\
4870^{\circ}\end{array}$ \\
\hline $\begin{array}{l}74 . \\
76 . \\
69 . \\
80 . \\
74 .\end{array}$ & $\begin{array}{l}440^{\circ} \\
420^{\circ} \\
380^{\circ} \\
410^{\circ} \\
40\end{array}$ & $\begin{array}{r}0.00 \\
.07 \\
.02 \\
0.00 \\
0.00\end{array}$ & $\begin{array}{l}52 . \\
50 . \\
65 . \\
73 . \\
51 .\end{array}$ & $\begin{array}{l}.22 \\
.20 \\
.27 \\
.32 \\
.19\end{array}$ & $\begin{array}{l}60 . \\
55 . \\
58 . \\
58 . \\
55 .\end{array}$ & $\begin{array}{l}71 . \\
67 . \\
45 . \\
57 . \\
57 .\end{array}$ & $\begin{array}{l}491 . \\
4590^{\circ} \\
6320^{\circ} \\
534 . \\
528^{\circ}\end{array}$ \\
\hline $\begin{array}{l}72 . \\
71 . \\
65 \\
75 \\
68 .\end{array}$ & $\begin{array}{l}40 . \\
36 . \\
35 \circ \\
38 . \\
41 .\end{array}$ & $\begin{array}{r}0.00 \\
0.00 \\
0.00 \\
0.00 \\
.06\end{array}$ & $\begin{array}{r}137 \\
72 \\
71 \\
84 \\
49\end{array}$ & $\begin{array}{l}.61 \\
.31 \\
.35 \\
.25 \\
.19\end{array}$ & $\begin{array}{l}57 . \\
58 . \\
61 . \\
60 . \\
59 .\end{array}$ & $\begin{array}{l}37 . \\
22 . \\
34 . \\
61 . \\
65 .\end{array}$ & $\begin{array}{l}708^{\circ} \\
468^{\circ} \\
5233^{\circ} \\
4160^{\circ}\end{array}$ \\
\hline
\end{tabular}


Table 6.--Climatic data, Antero Reservoir station,

\begin{tabular}{|c|c|c|c|c|c|c|c|}
\hline $\begin{array}{l}\text { DAY } \\
\text { OF } \\
\text { YEAR }\end{array}$ & MONTH & DAY & YEAR & $\begin{array}{l}\text { TEMF } \\
\text { MAX } \\
\text { (F) }\end{array}$ & $\begin{array}{l}\text { TURE } \\
\text { MIN } \\
\text { (F) }\end{array}$ & $\begin{array}{l}\text { DRY } \\
\text { BULA } \\
\text { (F) }\end{array}$ & $\begin{array}{l}\text { WET } \\
\text { BULB } \\
\text { (F) }\end{array}$ \\
\hline $\begin{array}{l}196 \\
197 \\
198 \\
199 \\
200\end{array}$ & $\begin{array}{l}7 \\
7 \\
7 \\
7 \\
7\end{array}$ & $\begin{array}{l}15 \\
16 \\
17 \\
18 \\
19\end{array}$ & $\begin{array}{l}77 \\
77 \\
17 \\
77 \\
17\end{array}$ & $\begin{array}{l}15 . \\
16 . \\
83 . \\
83 . \\
83 .\end{array}$ & $\begin{array}{l}37 \\
380^{\circ} \\
410^{\circ} \\
43{ }^{\circ}\end{array}$ & $\begin{array}{l}47 . \\
51 . \\
54 . \\
58 . \\
57 .\end{array}$ & $\begin{array}{l}46 . \\
48, \\
50 \\
54 . \\
53 .\end{array}$ \\
\hline $\begin{array}{l}201 \\
202 \\
203 \\
204 \\
205\end{array}$ & $\begin{array}{l}7 \\
7 \\
7 \\
7 \\
7\end{array}$ & $\begin{array}{l}20 \\
21 \\
22 \\
23 \\
24\end{array}$ & $\begin{array}{l}77 \\
77 \\
77 \\
77 \\
77\end{array}$ & $\begin{array}{l}80 . \\
69, \\
69 . \\
73 . \\
72 .\end{array}$ & $\begin{array}{l}45^{\circ} \\
460^{\circ} \\
44 \circ^{\circ} \\
40^{\circ}\end{array}$ & $\begin{array}{l}56 . \\
53 . \\
57 \\
550^{\circ} \\
50\end{array}$ & $\begin{array}{l}54 . \\
51 . \\
54 . \\
53 . \\
50 .\end{array}$ \\
\hline $\begin{array}{l}206 \\
207 \\
208 \\
209 \\
210\end{array}$ & $\begin{array}{l}7 \\
7 \\
7 \\
7 \\
7\end{array}$ & $\begin{array}{l}25 \\
26 \\
27 \\
28 \\
29\end{array}$ & $\begin{array}{l}77 \\
77 \\
77 \\
77 \\
77\end{array}$ & $\begin{array}{l}10 . \\
65 . \\
71 . \\
72 . \\
12 .\end{array}$ & $\begin{array}{l}460^{\circ} \\
480^{\circ} \\
440^{\circ} \\
42{ }^{\circ}\end{array}$ & $\begin{array}{l}54 . \\
52 . \\
50 . \\
53 . \\
52 .\end{array}$ & $\begin{array}{l}54 . \\
51 . \\
49 . \\
50 . \\
49 .\end{array}$ \\
\hline $\begin{array}{l}211 \\
212 \\
213 \\
214 \\
215\end{array}$ & $\begin{array}{l}7 \\
7 \\
8 \\
8 \\
8\end{array}$ & $\begin{array}{r}30 \\
31 \\
1 \\
2 \\
3\end{array}$ & $\begin{array}{l}17 \\
17 \\
17 \\
17 \\
17\end{array}$ & $\begin{array}{l}16 . \\
16 . \\
75 . \\
76 . \\
77 .\end{array}$ & $\begin{array}{l}45{ }^{\circ} \\
35 \circ^{\circ} \\
44^{\circ} \\
37{ }^{\circ}\end{array}$ & $\begin{array}{l}57 . \\
470^{\circ} \\
470^{\circ} \\
50 .\end{array}$ & $\begin{array}{l}52 . \\
44{ }^{\circ} \\
47 \\
45 \\
45\end{array}$ \\
\hline $\begin{array}{l}216 \\
217 \\
218 \\
219 \\
220\end{array}$ & $\begin{array}{l}8 \\
8 \\
8 \\
8 \\
8\end{array}$ & $\begin{array}{l}4 \\
5 \\
6 \\
7 \\
8\end{array}$ & $\begin{array}{l}77 \\
77 \\
77 \\
77 \\
77\end{array}$ & $\begin{array}{l}18 . \\
14 . \\
12 . \\
77 . \\
78 .\end{array}$ & $\begin{array}{l}400^{\circ} \\
47 \circ^{\circ} \\
39^{\circ} \\
4{ }^{\circ}\end{array}$ & $\begin{array}{l}53 . \\
52 . \\
56 . \\
49 . \\
50 .\end{array}$ & $\begin{array}{l}46 . \\
51 \\
53 \\
46 \\
48\end{array}$ \\
\hline
\end{tabular}


May-September 1977, 1978, and 1979--Continued

\begin{tabular}{|c|c|c|c|c|c|c|c|}
\hline $\begin{array}{l}\text { PAN } \\
\text { MAX } \\
\text { (F) }\end{array}$ & $\begin{array}{l}\text { WATER } \\
\text { MIN } \\
\text { (F) }\end{array}$ & $\begin{array}{l}\text { PKECIP } \\
\text { (IN.) }\end{array}$ & $\begin{array}{l}\text { WIND } \\
\text { (MI./DAY) }\end{array}$ & $\begin{array}{l}\text { PAN } \\
\text { EVAP } \\
\text { (IN.) }\end{array}$ & $\begin{array}{l}\text { DAII } \\
\text { TEMP } \\
\text { (F) }\end{array}$ & $\begin{array}{l}\text { Y AVG } \\
\text { REL HUM } \\
\text { (PERCENT) }\end{array}$ & $\begin{array}{l}\text { SOLAR } \\
\text { RAD } \\
\text { (LY.) }\end{array}$ \\
\hline $\begin{array}{l}70 . \\
71 . \\
77 \\
74 . \\
81 .\end{array}$ & $\begin{array}{l}41 . \\
40 \\
43 \\
46 \\
46 .\end{array}$ & $\begin{array}{l}.09 \\
0.00 \\
0.00 \\
0.00 \\
0.00\end{array}$ & $\begin{array}{l}49 . \\
56 . \\
54 . \\
51 . \\
68 .\end{array}$ & $\begin{array}{l}.21 \\
.49 \\
.25 \\
.39 \\
.37\end{array}$ & $\begin{array}{l}58 . \\
64 . \\
64 . \\
67 . \\
65 .\end{array}$ & $\begin{array}{l}95 . \\
83 . \\
49 . \\
41 . \\
61 .\end{array}$ & $\begin{array}{l}431 . \\
560 . \\
446 . \\
661 . \\
510 .\end{array}$ \\
\hline $\begin{array}{l}77 . \\
66 . \\
62 . \\
74 . \\
74 .\end{array}$ & $\begin{array}{l}46 . \\
46 . \\
470^{\circ} \\
48 \\
46 .\end{array}$ & $\begin{array}{r}.18 \\
1.38 \\
.23 \\
.06 \\
.33\end{array}$ & $\begin{array}{l}40 . \\
62 . \\
39 . \\
53 . \\
41 .\end{array}$ & $\begin{array}{l}.49 \\
.18 \\
.11 \\
.46 \\
.40\end{array}$ & $\begin{array}{l}55 . \\
56 . \\
60 . \\
60 . \\
58 .\end{array}$ & $\begin{array}{l}89 . \\
91 . \\
81 . \\
77 . \\
85 .\end{array}$ & $\begin{array}{l}253^{\circ} \\
2744^{\circ} \\
495^{\circ} \\
480^{\circ} \\
372^{\circ}\end{array}$ \\
\hline $\begin{array}{l}70 . \\
66 . \\
75 . \\
70 . \\
71 .\end{array}$ & $\begin{array}{l}46 . \\
47 . \\
46 . \\
45 . \\
46 .\end{array}$ & $\begin{array}{r}.74 \\
.07 \\
.19 \\
.07 \\
0.00\end{array}$ & $\begin{array}{l}46 . \\
51 . \\
56 . \\
41 . \\
37 .\end{array}$ & $\begin{array}{l}.17 \\
.21 \\
.22 \\
.23 \\
.27\end{array}$ & $\begin{array}{l}56 . \\
56 . \\
57 . \\
58 . \\
61 .\end{array}$ & $\begin{array}{l}89 . \\
79 . \\
69 . \\
61 . \\
53 .\end{array}$ & $\begin{array}{l}245 . \\
474 . \\
456 . \\
550^{\circ} \\
639^{\circ}\end{array}$ \\
\hline $\begin{array}{l}79 . \\
74 \circ^{\circ} \\
75 \circ^{\circ} \\
78 . \\
76 .\end{array}$ & $\begin{array}{l}47 . \\
41 . \\
41 . \\
42 . \\
42 .\end{array}$ & $\begin{array}{l}0.00 \\
0.00 \\
0.00 \\
0.00 \\
0.00\end{array}$ & $\begin{array}{l}67 . \\
500^{\circ} \\
370^{\circ} \\
34 \circ^{\circ} \\
43 .\end{array}$ & $\begin{array}{l}.43 \\
.32 \\
.24 \\
.36 \\
.25\end{array}$ & $\begin{array}{l}64 . \\
58 . \\
61 . \\
60 \\
61 .\end{array}$ & $\begin{array}{l}40 . \\
570^{\circ} \\
49 . \\
88 . \\
72 .\end{array}$ & $\begin{array}{l}658 . \\
672 . \\
647 . \\
629 . \\
409 .\end{array}$ \\
\hline $\begin{array}{l}78 . \\
69 . \\
73 . \\
73 . \\
75 .\end{array}$ & $\begin{array}{l}47 . \\
430^{\circ} \\
46 . \\
440^{\circ} \\
43 .\end{array}$ & $\begin{array}{r}0.00 \\
.07 \\
0.00 \\
0.00 \\
0.00\end{array}$ & $\begin{array}{l}45 . \\
570^{\circ} \\
62 . \\
49 . \\
79 .\end{array}$ & $\begin{array}{l}.18 \\
.22 \\
.34 \\
.35 \\
.17\end{array}$ & $\begin{array}{l}57 . \\
59 . \\
62 . \\
62 . \\
62 .\end{array}$ & $\begin{array}{l}71 . \\
67 . \\
53 . \\
45 . \\
69 .\end{array}$ & $\begin{array}{l}382 . \\
464^{\circ} \\
560^{\circ} \\
575^{\circ} \\
419^{\circ}\end{array}$ \\
\hline
\end{tabular}


Table 6.--Climatic data, Antero Reservoir station,

\begin{tabular}{|c|c|c|c|c|c|c|c|}
\hline $\begin{array}{r}\text { DAY } \\
\text { OF } \\
\text { YEAR }\end{array}$ & MONTH & DAY & YEAR & $\begin{array}{l}\text { TEM } \\
\text { MAX } \\
\text { (F) }\end{array}$ & $\begin{array}{l}\text { URE } \\
\text { MIN } \\
\text { (F) }\end{array}$ & $\begin{array}{l}\text { DRY } \\
\text { BULB } \\
\text { (F) }\end{array}$ & $\begin{array}{l}\text { WET } \\
\text { BULB } \\
\text { (F) }\end{array}$ \\
\hline $\begin{array}{l}221 \\
222 \\
223 \\
224 \\
225\end{array}$ & $\begin{array}{l}8 \\
8 \\
8 \\
8 \\
8\end{array}$ & $\begin{array}{r}9 \\
10 \\
11 \\
12 \\
13\end{array}$ & $\begin{array}{l}77 \\
77 \\
77 \\
77 \\
77\end{array}$ & $\begin{array}{l}16 . \\
78 . \\
72 . \\
71 . \\
15 .\end{array}$ & $\begin{array}{l}43 . \\
490^{\circ} \\
420^{\circ} \\
40 . \\
40 .\end{array}$ & $\begin{array}{l}48 \\
54 \\
50 \\
49 \\
52\end{array}$ & $\begin{array}{l}47 . \\
51 . \\
490^{\circ} \\
47^{\circ} \\
47 .\end{array}$ \\
\hline $\begin{array}{l}226 \\
227 \\
228 \\
229 \\
230\end{array}$ & $\begin{array}{l}8 \\
8 \\
8 \\
8 \\
8\end{array}$ & $\begin{array}{l}14 \\
15 \\
16 \\
17 \\
18\end{array}$ & $\begin{array}{l}77 \\
77 \\
77 \\
77 \\
77\end{array}$ & $\begin{array}{l}74 . \\
15 . \\
70 . \\
70 . \\
64 .\end{array}$ & $\begin{array}{l}39 . \\
48 . \\
50 . \\
49 . \\
52 .\end{array}$ & $\begin{array}{l}52 . \\
51 \\
54 \\
53 \\
58 \\
\end{array}$ & $\begin{array}{l}49 . \\
51 . \\
53 . \\
53 . \\
54 .\end{array}$ \\
\hline $\begin{array}{l}231 \\
232 \\
233 \\
234 \\
235\end{array}$ & $\begin{array}{l}8 \\
8 \\
8 \\
8 \\
8\end{array}$ & $\begin{array}{l}19 \\
20 \\
21 \\
22 \\
23\end{array}$ & $\begin{array}{l}77 \\
77 \\
77 \\
77 \\
77\end{array}$ & $\begin{array}{l}70 . \\
73 . \\
74 . \\
69 . \\
13 .\end{array}$ & $\begin{array}{l}450^{\circ} \\
480^{\circ} \\
460^{\circ} \\
440^{\circ} \\
41 .\end{array}$ & $\begin{array}{l}50 . \\
51 . \\
470^{\circ} \\
530^{\circ} \\
48 .\end{array}$ & $\begin{array}{l}50 . \\
50 . \\
47 \\
50 \\
47 .\end{array}$ \\
\hline $\begin{array}{l}236 \\
237 \\
238 \\
239 \\
240\end{array}$ & $\begin{array}{l}8 \\
8 \\
8 \\
8 \\
8\end{array}$ & $\begin{array}{l}24 \\
25 \\
26 \\
27 \\
28\end{array}$ & $\begin{array}{l}77 \\
77 \\
77 \\
77 \\
17\end{array}$ & $\begin{array}{l}15 . \\
12 . \\
70 . \\
15 . \\
62 .\end{array}$ & $\begin{array}{l}40 . \\
420^{\circ} \\
460^{\circ} \\
440^{\circ} \\
33 .\end{array}$ & $\begin{array}{l}49 . \\
55 . \\
58 . \\
51 . \\
37 .\end{array}$ & $\begin{array}{l}47 \\
51 \\
51 \\
48 \\
37\end{array}$ \\
\hline $\begin{array}{l}241 \\
242 \\
243 \\
244 \\
245\end{array}$ & $\begin{array}{l}8 \\
8 \\
8 \\
9 \\
9\end{array}$ & $\begin{array}{r}29 \\
30 \\
31 \\
1 \\
2\end{array}$ & $\begin{array}{l}77 \\
77 \\
77 \\
77 \\
77\end{array}$ & $\begin{array}{l}64 . \\
13 . \\
12 . \\
14 . \\
15 .\end{array}$ & $\begin{array}{l}32 . \\
34{ }^{\circ} \\
350^{\circ} \\
35 . \\
38 .\end{array}$ & $\begin{array}{l}40 . \\
390^{\circ} \\
470^{\circ} \\
540^{\circ}\end{array}$ & $\begin{array}{l}39 \\
39 \\
42 \\
48 \\
45\end{array}$ \\
\hline
\end{tabular}


May-September 1977, 1978, and 1979--Continued

\begin{tabular}{|c|c|c|c|c|c|c|c|}
\hline $\begin{array}{l}\text { PAN } \\
\text { MAX } \\
\text { (F) }\end{array}$ & $\begin{array}{l}\text { WATER } \\
\text { MIN } \\
\text { (F) }\end{array}$ & $\begin{array}{l}\text { PRECIP } \\
\text { (IN.) }\end{array}$ & $\begin{array}{l}\text { WIND } \\
\text { (MI./DAY) }\end{array}$ & $\begin{array}{l}\text { PAN } \\
\text { EVAP } \\
\text { (IN.) }\end{array}$ & $\begin{array}{l}\text { DAIL } \\
\text { TEMP } \\
\text { (F) }\end{array}$ & $\begin{array}{l}\text { YVG } \\
\text { REL HUM } \\
\text { (PERCENT) }\end{array}$ & $\begin{array}{l}\text { SOLAR } \\
\text { RAD } \\
\text { (LY, })\end{array}$ \\
\hline $\begin{array}{l}69 . \\
76 . \\
69 . \\
68 . \\
73 .\end{array}$ & $\begin{array}{l}44 \\
44 \\
45 \\
43 \\
43\end{array}$ & $\begin{array}{l}.05 \\
0.00 \\
0.00 \\
0.00 \\
0.00\end{array}$ & $\begin{array}{l}48 . \\
61 \\
43 \\
38 \\
45\end{array}$ & $\begin{array}{l}.23 \\
.25 \\
.15 \\
.24 \\
.19\end{array}$ & $\begin{array}{l}60 . \\
59 . \\
56 . \\
56 . \\
58 .\end{array}$ & $\begin{array}{l}94 . \\
85 . \\
75 . \\
56 . \\
69 .\end{array}$ & $\begin{array}{l}459^{\circ} \\
444^{\circ} \\
348^{\circ} \\
500 \\
399^{\circ}\end{array}$ \\
\hline $\begin{array}{l}69 . \\
75 . \\
70 . \\
68 . \\
62 .\end{array}$ & $\begin{array}{l}42 . \\
430^{\circ} \\
450^{\circ} \\
490^{\circ} \\
49 .\end{array}$ & $\begin{array}{l}.09 \\
.03 \\
.52 \\
.26 \\
.05\end{array}$ & $\begin{array}{l}47 . \\
62 . \\
40 . \\
56 . \\
57 .\end{array}$ & $\begin{array}{l}.22 \\
.21 \\
.10 \\
.06 \\
.15\end{array}$ & $\begin{array}{l}57 . \\
62 . \\
57 . \\
58 . \\
62 .\end{array}$ & $\begin{array}{l}68 . \\
73 . \\
95 . \\
86 . \\
72 .\end{array}$ & $\begin{array}{l}511 . \\
300 . \\
239 \\
161 . \\
305 .\end{array}$ \\
\hline $\begin{array}{l}66 . \\
72 . \\
74 . \\
67 . \\
73 .\end{array}$ & $\begin{array}{l}46 . \\
460^{\circ} \\
470^{\circ} \\
430^{\circ}\end{array}$ & $\begin{array}{r}.02 \\
.01 \\
0.00 \\
.02 \\
.07\end{array}$ & $\begin{array}{l}57 . \\
50 . \\
52 . \\
69 . \\
52 .\end{array}$ & $\begin{array}{l}.19 \\
.28 \\
.20 \\
.45 \\
.16\end{array}$ & $\begin{array}{l}59 . \\
60 . \\
56 . \\
61 . \\
62 .\end{array}$ & $\begin{array}{l}83 . \\
68 . \\
75 . \\
58 . \\
68 .\end{array}$ & $\begin{array}{l}405 . \\
5470^{\circ} \\
425^{\circ} \\
5930^{\circ}\end{array}$ \\
\hline $\begin{array}{l}71 . \\
67 . \\
67 . \\
71 . \\
64 .\end{array}$ & $\begin{array}{l}43 . \\
42 . \\
430^{\circ} \\
41 . \\
34 .\end{array}$ & $\begin{array}{l}0.00 \\
.03 \\
0.00 \\
.04 \\
0.00\end{array}$ & $\begin{array}{r}86 . \\
81 . \\
119 . \\
71 . \\
56 .\end{array}$ & $\begin{array}{r}.31 \\
.25 \\
.37 \\
.31 \\
.18\end{array}$ & $\begin{array}{l}56 . \\
60 . \\
62 . \\
57 . \\
52 .\end{array}$ & $\begin{array}{l}74 . \\
63 . \\
50 . \\
39 . \\
59 .\end{array}$ & $\begin{array}{l}419 . \\
492 . \\
467 \\
*\end{array}$ \\
\hline $\begin{array}{l}64 . \\
72 . \\
67 . \\
66 . \\
71\end{array}$ & $\begin{array}{l}34 . \\
35 . \\
37 \\
37 \\
42 .\end{array}$ & $\begin{array}{r}0.00 \\
0.00 \\
.02 \\
0.00 \\
0.00\end{array}$ & $\begin{array}{l}69 . \\
97 . \\
92 . \\
69 . \\
48 .\end{array}$ & $\begin{array}{r}.32 \\
.25 \\
.24 \\
.31 \\
.16\end{array}$ & $\begin{array}{l}56 . \\
55 . \\
51 . \\
59 . \\
57 .\end{array}$ & $\begin{array}{l}50 . \\
60 . \\
58 . \\
51 . \\
63 .\end{array}$ & $\begin{array}{l}* * * * \\
* * * \\
408 . \\
330 .\end{array}$ \\
\hline
\end{tabular}


Table 6.--Climatic data, Antero Reservoir station,

\begin{tabular}{|c|c|c|c|c|c|c|c|}
\hline $\begin{array}{r}\text { DAY } \\
\text { OF } \\
\text { YEAR }\end{array}$ & MONTH & DAY & YEAR & $\begin{array}{l}\text { TEMF } \\
\text { MAX } \\
\text { (F) }\end{array}$ & $\begin{array}{l}\text { URE } \\
\text { MIN } \\
\text { (F) }\end{array}$ & $\begin{array}{l}\text { DRY } \\
\text { BULB } \\
\text { (F) }\end{array}$ & $\begin{array}{l}\text { WET } \\
\text { BULB } B \\
\text { (F) }\end{array}$ \\
\hline $\begin{array}{l}246 \\
247 \\
248 \\
249 \\
250\end{array}$ & $\begin{array}{l}9 \\
9 \\
9 \\
9 \\
9\end{array}$ & $\begin{array}{l}3 \\
4 \\
5 \\
6 \\
7\end{array}$ & $\begin{array}{l}77 \\
77 \\
77 \\
77 \\
77\end{array}$ & $\begin{array}{l}14 . \\
12 . \\
680^{\circ} \\
74 . \\
15 .\end{array}$ & $\begin{array}{l}390^{\circ} \\
380^{\circ} \\
34{ }^{\circ} \\
34^{\circ} \\
36 .\end{array}$ & $\begin{array}{l}47 . \\
48 . \\
45 \circ \\
40 . \\
45 .\end{array}$ & $\begin{array}{l}45 \\
46 \\
44{ }^{\circ} \\
40 \\
45\end{array}$ \\
\hline $\begin{array}{l}251 \\
252 \\
253 \\
254 \\
255\end{array}$ & $\begin{array}{l}9 \\
9 \\
9 \\
9 \\
9\end{array}$ & $\begin{array}{r}8 \\
9 \\
10 \\
11 \\
12\end{array}$ & $\begin{array}{l}17 \\
77 \\
17 \\
77 \\
77\end{array}$ & $\begin{array}{l}78 . \\
15 . \\
70 . \\
12 . \\
61 .\end{array}$ & $\begin{array}{l}33 . \\
39 . \\
32 . \\
31 . \\
43 .\end{array}$ & $\begin{array}{l}43{ }^{\circ} \\
44 \circ^{\circ} \\
460^{\circ} \\
480^{\circ} \\
35^{\circ}\end{array}$ & $\begin{array}{l}42 \\
41 \\
43 \\
46 \\
33\end{array}$ \\
\hline $\begin{array}{l}256 \\
257 \\
258 \\
259 \\
260\end{array}$ & $\begin{array}{l}9 \\
9 \\
9 \\
9 \\
9\end{array}$ & $\begin{array}{l}13 \\
14 \\
15 \\
16 \\
17\end{array}$ & $\begin{array}{l}77 \\
17 \\
77 \\
77 \\
17\end{array}$ & $\begin{array}{l}63 . \\
63 . \\
67 . \\
65 . \\
63 .\end{array}$ & $\begin{array}{l}32 . \\
23 . \\
28 . \\
29 . \\
33 .\end{array}$ & $\begin{array}{l}35 . \\
290^{\circ} \\
510^{\circ} \\
36 . \\
49 .\end{array}$ & $\begin{array}{l}33 . \\
29 . \\
45 . \\
35 . \\
42 .\end{array}$ \\
\hline $\begin{array}{l}261 \\
262 \\
263 \\
264 \\
265\end{array}$ & $\begin{array}{l}9 \\
9 \\
9 \\
9 \\
9\end{array}$ & $\begin{array}{l}18 \\
19 \\
20 \\
21 \\
22\end{array}$ & $\begin{array}{l}77 \\
77 \\
77 \\
77 \\
77\end{array}$ & $\begin{array}{l}62 . \\
66 . \\
69 . \\
70 . \\
65 .\end{array}$ & $\begin{array}{l}23 . \\
26 . \\
31 . \\
35 . \\
24 .\end{array}$ & $\begin{array}{l}33 . \\
32 . \\
40 . \\
42 . \\
32 .\end{array}$ & $\begin{array}{l}31 \\
30 \\
40 \\
38 \\
32\end{array}$ \\
\hline $\begin{array}{l}266 \\
267 \\
268 \\
269 \\
270\end{array}$ & $\begin{array}{l}9 \\
9 \\
9 \\
9 \\
9\end{array}$ & $\begin{array}{l}23 \\
24 \\
25 \\
26 \\
27\end{array}$ & $\begin{array}{l}77 \\
77 \\
77 \\
77 \\
77\end{array}$ & $\begin{array}{l}66 . \\
55 . \\
66 . \\
68 . \\
63 .\end{array}$ & $\begin{array}{l}31 . \\
31 . \\
36 . \\
32 . \\
23 .\end{array}$ & $\begin{array}{l}45 . \\
48 . \\
50 \\
48 . \\
32 .\end{array}$ & $\begin{array}{l}38 \\
38 \\
40 \\
38 \\
32\end{array}$ \\
\hline
\end{tabular}


May-September 1977, 1978, and 1979--Continued

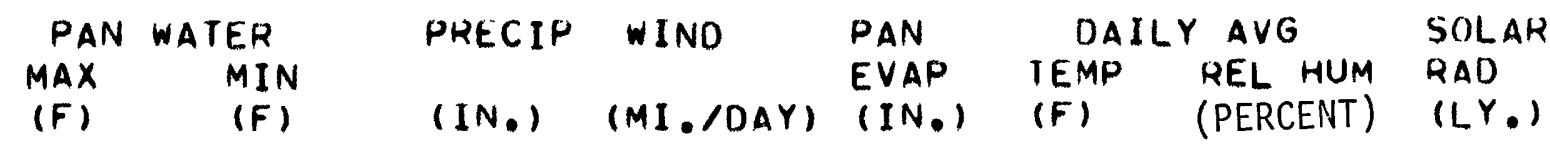

\begin{tabular}{|c|c|c|c|c|c|c|c|}
\hline $\begin{array}{l}66 . \\
65 . \\
63 . \\
63 . \\
75 .\end{array}$ & $\begin{array}{l}40 . \\
40 . \\
40 \\
38 \\
38 .\end{array}$ & $\begin{array}{r}0.00 \\
.07 \\
.05 \\
.12 \\
0.00\end{array}$ & $\begin{array}{l}56 . \\
43 . \\
40 . \\
33 . \\
28 .\end{array}$ & $\begin{array}{l}.21 \\
.12 \\
.27 \\
.25 \\
.21\end{array}$ & $\begin{array}{l}56 . \\
53 . \\
56 . \\
54 . \\
56 .\end{array}$ & $\begin{array}{l}71 . \\
73 . \\
63 \\
54 . \\
04\end{array}$ & $\begin{array}{l}372 . \\
369 . \\
461 . \\
501 .\end{array}$ \\
\hline
\end{tabular}

\begin{tabular}{|c|c|c|c|c|c|c|c|}
\hline $\begin{array}{l}75 . \\
64 . \\
64 . \\
63 . \\
52 .\end{array}$ & $\begin{array}{l}39 . \\
37 . \\
35 . \\
35 . \\
42 .\end{array}$ & $\begin{array}{r}0.00 \\
0.00 \\
0.00 \\
.17 \\
0.00\end{array}$ & $\begin{array}{l}97 . \\
52 . \\
67 . \\
49 . \\
52 .\end{array}$ & $\begin{array}{l}.26 \\
.21 \\
.36 \\
.08 \\
.18\end{array}$ & $\begin{array}{l}58 . \\
57 . \\
53 . \\
56 . \\
50 .\end{array}$ & $\begin{array}{l}41 . \\
60 . \\
64 . \\
94 . \\
85 .\end{array}$ & $\begin{array}{l}397^{\circ} \\
396 . \\
323 . \\
181 . \\
251 .\end{array}$ \\
\hline $\begin{array}{l}57 . \\
65 . \\
64 . \\
64 . \\
60 .\end{array}$ & $\begin{array}{l}35 . \\
31 . \\
30 \\
34^{\circ} \\
34^{\circ}\end{array}$ & $\begin{array}{l}0.00 \\
0.00 \\
.04 \\
0.00 \\
0.00\end{array}$ & $\begin{array}{r}41 . \\
55 . \\
91 . \\
89 . \\
119 .\end{array}$ & $\begin{array}{l}.10 \\
.22 \\
.27 \\
.25 \\
.24\end{array}$ & $\begin{array}{l}46 . \\
47 . \\
53 . \\
48 . \\
53 .\end{array}$ & $\begin{array}{l}84 . \\
98 . \\
66 . \\
56 . \\
40 .\end{array}$ & $\begin{array}{l}449^{\circ} \\
420 \\
456 \\
449 \\
481\end{array}$ \\
\hline $\begin{array}{l}62 . \\
64 . \\
64 . \\
66 . \\
63 .\end{array}$ & $\begin{array}{l}32 . \\
31 . \\
33 . \\
34 . \\
33 .\end{array}$ & $\begin{array}{l}0.00 \\
0.00 \\
0.00 \\
0.00 \\
0.00\end{array}$ & $\begin{array}{r}62 . \\
66 . \\
107 . \\
68 . \\
113 .\end{array}$ & $\begin{array}{r}.36 \\
.26 \\
.32 \\
.31 \\
.30\end{array}$ & $\begin{array}{l}49 . \\
51 . \\
65 . \\
53 . \\
49 .\end{array}$ & $\begin{array}{l}37 . \\
53 . \\
48 . \\
30 . \\
52 .\end{array}$ & $\begin{array}{l}472 . \\
492 . \\
491 \\
472 . \\
459\end{array}$ \\
\hline $\begin{array}{l}62 \\
64 \\
61\end{array}$ & $\begin{array}{l}32 . \\
31 . \\
36 . \\
34 \\
33 .\end{array}$ & $\begin{array}{l}0.00 \\
0.00 \\
0.00 \\
0.00 \\
0.00\end{array}$ & $\begin{array}{r}184 . \\
132 \\
112 . \\
85 \\
81 .\end{array}$ & $\begin{array}{l}.31 \\
.36 \\
.29 \\
.21 \\
.27\end{array}$ & $\begin{array}{l}50 . \\
53 . \\
54 . \\
51 . \\
49 .\end{array}$ & $\begin{array}{l}35 . \\
25 . \\
39 . \\
52 . \\
54 .\end{array}$ & $\begin{array}{l}471 \\
472 \\
387 \\
469 \\
395\end{array}$ \\
\hline
\end{tabular}


Table 6.--Climatic data, Antero Reservoir station,

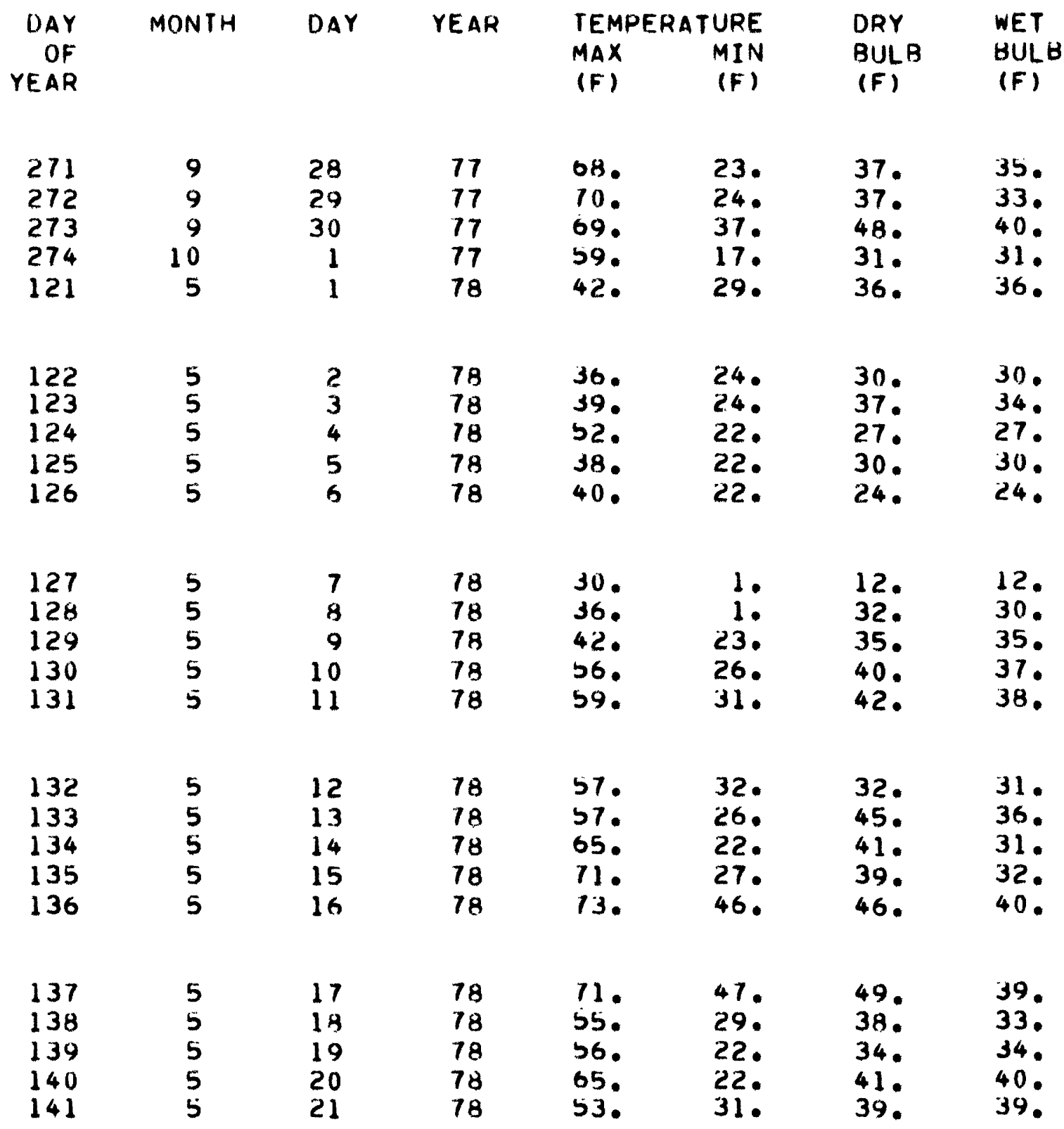


May-September 1977, 1978, and 1979--Continued

\begin{tabular}{|c|c|c|c|c|c|c|c|}
\hline $\begin{array}{l}\text { PAN } \\
\text { MAX } \\
\text { (F) }\end{array}$ & $\begin{array}{l}\text { WATER } \\
\text { MIN } \\
\text { (F) }\end{array}$ & $\begin{array}{l}\text { PRECIP } \\
\text { (IN.) }\end{array}$ & $\begin{array}{l}\text { WIND } \\
(M I . / D \Delta Y)\end{array}$ & $\begin{array}{l}\text { PAN } \\
\text { EVAP } \\
\text { (IN.) }\end{array}$ & $\begin{array}{l}\text { DA } \\
\text { TEMP } \\
\text { (F) }\end{array}$ & $\begin{array}{l}\text { Y AVG } \\
\text { REL HUM } \\
\text { (PERCENT) }\end{array}$ & $\begin{array}{l}\text { SOLAR } \\
\text { RAD } \\
(L Y,)\end{array}$ \\
\hline $\begin{array}{l}60 . \\
640^{\circ} \\
600^{\circ} \\
55^{\circ} \\
39 .\end{array}$ & $\begin{array}{l}33 . \\
32 . \\
32 . \\
29 . \\
37 .\end{array}$ & $\begin{array}{r}0.00 \\
0.00 \\
0.00 \\
0.00 \\
.01\end{array}$ & $\begin{array}{r}33 . \\
125 . \\
120 . \\
58 . \\
93 .\end{array}$ & $\begin{array}{l}.16 \\
.34 \\
.22 \\
.22 \\
.08\end{array}$ & $\begin{array}{r}53 . \\
51 . \\
51 . \\
41 . \\
504\end{array}$ & $\begin{array}{l}51 . \\
48 . \\
47 \\
57 \\
\$ 4\end{array}$ & $\begin{array}{l}366 \\
376 \\
330 \\
452 \\
\end{array}$ \\
\hline $\begin{array}{l}37 . \\
50 . \\
59 . \\
48 . \\
50 .\end{array}$ & $\begin{array}{l}32 . \\
34 . \\
32 \\
32 \\
32 .\end{array}$ & $\begin{array}{r}.05 \\
0.00 \\
.05 \\
0.00 \\
.04\end{array}$ & $\begin{array}{r}47 . \\
96 . \\
78 . \\
132 . \\
101 .\end{array}$ & $\begin{array}{l}.06 \\
.26 \\
.02 \\
.04 \\
.03\end{array}$ & $\begin{array}{l}26 . \\
35 . \\
24 . \\
25 . \\
20 .\end{array}$ & $\begin{array}{l}75 . \\
57 . \\
70 . \\
68 . \\
80 .\end{array}$ & $\begin{array}{l}* * * * \\
* * * 4 \\
* * * * \\
* * * * \\
* * * *\end{array}$ \\
\hline $\begin{array}{l}41 . \\
50 . \\
52 . \\
66 . \\
68 .\end{array}$ & $\begin{array}{l}27 . \\
27 . \\
37 . \\
36 . \\
36 .\end{array}$ & $\begin{array}{l}.03 \\
0.00 \\
0.00 \\
0.00 \\
0.00\end{array}$ & $\begin{array}{l}137 . \\
105 . \\
66 . \\
150 . \\
209 .\end{array}$ & $\begin{array}{r}.20 \\
.99 \\
.33 \\
.39 \\
.30\end{array}$ & $\begin{array}{l}20 . \\
29 . \\
36 . \\
41 . \\
41 .\end{array}$ & $\begin{array}{l}56 . \\
51 . \\
31 . \\
39 . \\
50 .\end{array}$ & $\begin{array}{l}* 4 * \\
4 \% * \\
700 . \\
580 .\end{array}$ \\
\hline $\begin{array}{l}640^{\circ} \\
610^{\circ} \\
730^{\circ} \\
770^{\circ} \\
75 .\end{array}$ & $\begin{array}{l}34 . \\
32 . \\
37 . \\
36 . \\
37 .\end{array}$ & $\begin{array}{l}0.00 \\
0.00 \\
0.00 \\
0.00 \\
0.00\end{array}$ & $\begin{array}{r}145 . \\
78 \\
68^{\circ} \\
80 \\
132^{\circ}\end{array}$ & $\begin{array}{l}.35 \\
.32 \\
.15 \\
.49 \\
.50\end{array}$ & $\begin{array}{l}38 . \\
45 . \\
48 . \\
50 \\
40 .\end{array}$ & $\begin{array}{l}36 . \\
26 . \\
34 . \\
38 . \\
32 .\end{array}$ & $\begin{array}{l}724{ }^{\circ} \\
729^{\circ} \\
726^{\circ} \\
719^{\circ}\end{array}$ \\
\hline $\begin{array}{l}73 . \\
57 . \\
68 . \\
72 \\
55 .\end{array}$ & $\begin{array}{l}39 . \\
34: \\
32 \\
37 \\
34\end{array}$ & $\begin{array}{l}0.00 \\
0.00 \\
0.00 \\
0.00 \\
0.00\end{array}$ & $\begin{array}{l}163 . \\
107 . \\
99 . \\
66 . \\
95 .\end{array}$ & $\begin{array}{l}.25 \\
.39 \\
.16 \\
.23 \\
.14\end{array}$ & $\begin{array}{l}40 . \\
40 . \\
45 . \\
40 . \\
41 .\end{array}$ & $\begin{array}{l}39 . \\
40 . \\
31 . \\
78 . \\
74 .\end{array}$ & $\begin{array}{l}478^{\circ} \\
733^{\circ} \\
657^{\circ} \\
236 \\
359^{\circ}\end{array}$ \\
\hline
\end{tabular}


Table 6.--Climatic data, Antero Reservoir station,

\begin{tabular}{|c|c|c|c|c|c|c|c|}
\hline $\begin{array}{r}\text { DAY } \\
\text { OF } \\
\text { YEAR }\end{array}$ & MONTH & DAY & YEAR & $\begin{array}{l}\text { TEMF } \\
\text { MAX } \\
\text { (F) }\end{array}$ & $\begin{array}{l}\text { TURE } \\
\text { MIN } \\
\text { (F) }\end{array}$ & $\begin{array}{l}\text { DRY } \\
\text { BULB } \\
\text { (F) }\end{array}$ & $\begin{array}{l}\text { WET } \\
\text { BULB } \\
\text { (F) }\end{array}$ \\
\hline $\begin{array}{l}142 \\
143 \\
144 \\
145 \\
146\end{array}$ & $\begin{array}{l}5 \\
5 \\
5 \\
5 \\
5\end{array}$ & $\begin{array}{l}22 \\
23 \\
24 \\
25 \\
26\end{array}$ & $\begin{array}{l}78 \\
78 \\
78 \\
78 \\
78\end{array}$ & $\begin{array}{l}55 . \\
64 . \\
10 . \\
63 . \\
61 .\end{array}$ & $\begin{array}{l}280^{\circ} \\
24 \circ^{\circ} \\
270^{\circ} \\
250^{\circ}\end{array}$ & $\begin{array}{l}44 \circ^{\circ} \\
390^{\circ} \\
420^{\circ} \\
40\end{array}$ & $\begin{array}{l}38 \\
34 \\
36 \\
35 . \\
38\end{array}$ \\
\hline $\begin{array}{l}147 \\
148 \\
149 \\
150 \\
151\end{array}$ & $\begin{array}{l}5 \\
5 \\
5 \\
5 \\
5\end{array}$ & $\begin{array}{l}27 \\
28 \\
29 \\
30 \\
31\end{array}$ & $\begin{array}{l}78 \\
78 \\
78 \\
78 \\
78\end{array}$ & $\begin{array}{l}63 . \\
52 . \\
66 . \\
63 . \\
65 .\end{array}$ & $\begin{array}{l}34{ }^{\circ} \\
24{ }^{\circ} \\
220^{\circ} \\
350^{\circ}\end{array}$ & $\begin{array}{l}40 . \\
350^{\circ} \\
34{ }^{\circ} \\
53{ }^{\circ}\end{array}$ & $\begin{array}{l}40 \\
35 \\
33 \\
42 \\
38\end{array}$ \\
\hline $\begin{array}{l}152 \\
153 \\
154 \\
155 \\
156\end{array}$ & $\begin{array}{l}6 \\
6 \\
6 \\
6 \\
6\end{array}$ & $\begin{array}{l}1 \\
2 \\
3 \\
4 \\
5\end{array}$ & $\begin{array}{l}78 \\
78 \\
78 \\
78 \\
78\end{array}$ & $\begin{array}{l}63 . \\
66 . \\
65 . \\
59 . \\
57 .\end{array}$ & $\begin{array}{l}37 . \\
390^{\circ} \\
37 \\
350^{\circ}\end{array}$ & $\begin{array}{l}40 . \\
430^{\circ} \\
430^{\circ} \\
38 \\
35\end{array}$ & $\begin{array}{l}40 \\
43 \\
41 \\
38 \\
35\end{array}$ \\
\hline $\begin{array}{l}157 \\
158 \\
159 \\
160 \\
161\end{array}$ & $\begin{array}{l}6 \\
6 \\
6 \\
6 \\
6\end{array}$ & $\begin{array}{r}6 \\
7 \\
8 \\
9 \\
10\end{array}$ & $\begin{array}{l}18 \\
78 \\
78 \\
78 \\
78\end{array}$ & $\begin{array}{l}62 . \\
64 . \\
65 . \\
14 . \\
72 .\end{array}$ & $\begin{array}{l}33 . \\
31 . \\
29 . \\
41 . \\
50 .\end{array}$ & $\begin{array}{l}43 . \\
41 . \\
390^{\circ} \\
53 . \\
61 .\end{array}$ & $\begin{array}{l}43 \\
40 \\
39 \\
49 \\
47\end{array}$ \\
\hline $\begin{array}{l}162 \\
163 \\
164 \\
165 \\
166\end{array}$ & $\begin{array}{l}6 \\
6 \\
6 \\
6 \\
6\end{array}$ & $\begin{array}{l}11 \\
12 \\
13 \\
14 \\
15\end{array}$ & $\begin{array}{l}78 \\
78 \\
78 \\
78 \\
78\end{array}$ & $\begin{array}{l}72 . \\
72 . \\
75 . \\
76 \\
75 .\end{array}$ & $\begin{array}{l}32 . \\
33 \\
32 \\
40 \\
48\end{array}$ & $\begin{array}{l}49 . \\
50 \\
39 \\
50 \\
49\end{array}$ & $\begin{array}{l}450^{\circ} \\
460^{\circ} \\
50 \\
48\end{array}$ \\
\hline
\end{tabular}


May-September 1977, 1978, and 1979--Continued

\begin{tabular}{|c|c|c|c|c|c|c|c|}
\hline $\begin{array}{l}\text { PAN } \\
\text { MAX } \\
\text { (F) }\end{array}$ & $\begin{array}{l}\text { WATER } \\
\text { MIN } \\
\text { (F) }\end{array}$ & $\begin{array}{l}\text { PRECIP } \\
\left(I N_{0}\right)\end{array}$ & $\begin{array}{l}\text { WIND } \\
\text { (MI./DAY) }\end{array}$ & $\begin{array}{l}\text { PAN } \\
\text { EVAP } \\
\text { (IN.) }\end{array}$ & $\begin{array}{l}\text { DAI } \\
\text { IEMP } \\
\text { (F) }\end{array}$ & $\begin{array}{l}\text { Y AVG } \\
\text { REL HUM } \\
\text { (PERCENT) }\end{array}$ & $\begin{array}{l}\text { SOLAR } \\
\text { RAD } \\
(L Y,)\end{array}$ \\
\hline $\begin{array}{l}59 . \\
70 . \\
64 . \\
70 . \\
64 .\end{array}$ & $\begin{array}{l}37 . \\
37 . \\
39 . \\
37 . \\
36 .\end{array}$ & $\begin{array}{l}0.00 \\
0.00 \\
0.00 \\
0.00 \\
0.00\end{array}$ & $\begin{array}{r}90 . \\
106 . \\
121 . \\
107 . \\
89 .\end{array}$ & $\begin{array}{r}.34 \\
.31 \\
.43 \\
.35 \\
.24\end{array}$ & $\begin{array}{l}46 . \\
45 . \\
46 . \\
43 \\
41\end{array}$ & $\begin{array}{l}49 . \\
47 . \\
34 . \\
25 . \\
39 .\end{array}$ & $\begin{array}{l}595 . \\
520 . \\
719 . \\
592 . \\
\end{array}$ \\
\hline $\begin{array}{l}66 . \\
70 . \\
79 . \\
72 . \\
70 .\end{array}$ & $\begin{array}{l}37 . \\
36 . \\
36 . \\
36 . \\
37 .\end{array}$ & $\begin{array}{r}.15 \\
.01 \\
0.00 \\
0.00 \\
0.00\end{array}$ & $\begin{array}{l}74{ }^{\circ} \\
710^{\circ} \\
122^{\circ} \\
159^{\circ} \\
260^{\circ}\end{array}$ & $\begin{array}{l}.19 \\
.19 \\
.39 \\
.40 \\
.52\end{array}$ & $\begin{array}{l}40 . \\
39 . \\
43 . \\
51 . \\
45 .\end{array}$ & $\begin{array}{l}68 . \\
55 . \\
42 . \\
28 . \\
63 .\end{array}$ & $\begin{array}{l}324 . \\
472 . \\
631^{\circ} \\
564 . \\
530^{\circ}\end{array}$ \\
\hline $\begin{array}{l}66 . \\
73 . \\
70 . \\
57 . \\
64 .\end{array}$ & $\begin{array}{l}37 . \\
41 . \\
41 . \\
39 . \\
37 .\end{array}$ & $\begin{array}{r}.04 \\
0.00 \\
0.00 \\
.21 \\
.16\end{array}$ & $\begin{array}{r}96 . \\
104 . \\
71 . \\
59 . \\
67 .\end{array}$ & $\begin{array}{l}.28 \\
.33 \\
.09 \\
.15 \\
.28\end{array}$ & $\begin{array}{l}41 . \\
45 . \\
49 \\
42 . \\
38\end{array}$ & $\begin{array}{l}81 . \\
74 . \\
63 . \\
79 . \\
87 .\end{array}$ & $\begin{array}{l}320^{\circ} \\
449^{\circ} \\
4490^{\circ} \\
1945^{\circ}\end{array}$ \\
\hline $\begin{array}{l}72 . \\
68 . \\
75 \\
77 . \\
72 .\end{array}$ & $\begin{array}{l}37 . \\
37 . \\
39 . \\
41 . \\
45 .\end{array}$ & $\begin{array}{l}0.00 \\
.05 \\
0.00 \\
0.00 \\
0.00\end{array}$ & $\begin{array}{r}18 . \\
49 . \\
91 . \\
174 \\
120\end{array}$ & $\begin{array}{l}.20 \\
.30 \\
.41 \\
.34 \\
.70\end{array}$ & $\begin{array}{l}42 . \\
44 . \\
47 . \\
52 . \\
59 .\end{array}$ & $\begin{array}{l}64 . \\
630^{\circ} \\
58 . \\
55 \\
32 .\end{array}$ & $\begin{array}{l}550^{\circ} \\
363^{\circ} \\
608 \\
583^{\circ} \\
497^{\circ}\end{array}$ \\
\hline $\begin{array}{l}73 . \\
73 . \\
77 . \\
79 . \\
72 .\end{array}$ & $\begin{array}{l}43 . \\
430^{\circ} \\
41 . \\
41 . \\
43 .\end{array}$ & $\begin{array}{l}0.00 \\
0.00 \\
0.00 \\
0.00 \\
0.00\end{array}$ & $\begin{array}{r}87 . \\
102 . \\
85 . \\
162 . \\
170\end{array}$ & $\begin{array}{l}.37 \\
.37 \\
.43 \\
.59 \\
.62\end{array}$ & $\begin{array}{l}60 . \\
55 . \\
54 . \\
56 . \\
58 .\end{array}$ & $\begin{array}{l}22 . \\
54 . \\
48 . \\
45 . \\
36 .\end{array}$ & $\begin{array}{l}530 \\
458 \\
492 \\
487 \\
562\end{array}$ \\
\hline
\end{tabular}


Table 6.--Climatic data, Antero Reservoir station,

\begin{tabular}{|c|c|c|c|c|c|c|c|}
\hline $\begin{array}{l}\text { DAY } \\
\text { OF } \\
\text { YEAR }\end{array}$ & MONTH & DAY & YEAR & $\begin{array}{l}\text { TEMF } \\
\text { MAX } \\
\text { (F) }\end{array}$ & $\begin{array}{l}\text { TURE } \\
\text { MIN } \\
\text { (F) }\end{array}$ & $\begin{array}{l}\text { DRY } \\
\text { BULB } \\
\text { (F) }\end{array}$ & $\begin{array}{l}\text { WET } \\
\text { BULB } \\
\text { (F) }\end{array}$ \\
\hline $\begin{array}{l}167 \\
168 \\
169 \\
170 \\
171\end{array}$ & $\begin{array}{l}6 \\
6 \\
6 \\
6 \\
6\end{array}$ & $\begin{array}{l}16 \\
17 \\
18 \\
19 \\
20\end{array}$ & $\begin{array}{l}78 \\
78 \\
78 \\
78 \\
78\end{array}$ & $\begin{array}{l}73 . \\
73 . \\
11 . \\
72 . \\
75 .\end{array}$ & $\begin{array}{l}40 . \\
32 . \\
39 . \\
31 . \\
40 .\end{array}$ & $\begin{array}{l}45 \\
41 \\
61 \\
38 \\
46\end{array}$ & $\begin{array}{l}42 . \\
39{ }^{\circ} \\
47^{\circ} \\
38 \\
46\end{array}$ \\
\hline $\begin{array}{l}172 \\
173 \\
174 \\
175 \\
176\end{array}$ & $\begin{array}{l}6 \\
6 \\
6 \\
6 \\
6\end{array}$ & $\begin{array}{l}21 \\
22 \\
23 \\
24 \\
25\end{array}$ & $\begin{array}{l}78 \\
78 \\
78 \\
78 \\
78\end{array}$ & $\begin{array}{l}77 . \\
77 . \\
79 . \\
78 . \\
73 .\end{array}$ & $\begin{array}{l}35 . \\
37 \\
37 \\
55 \\
32\end{array}$ & $\begin{array}{l}47 . \\
440^{\circ} \\
460^{\circ} \\
50^{\circ} \\
4\end{array}$ & $\begin{array}{l}41 . \\
44 \\
46 . \\
64 \\
40\end{array}$ \\
\hline $\begin{array}{l}177 \\
178 \\
179 \\
180 \\
181\end{array}$ & $\begin{array}{l}6 \\
6 \\
6 \\
6 \\
6\end{array}$ & $\begin{array}{l}26 \\
27 \\
28 \\
29 \\
30\end{array}$ & $\begin{array}{l}78 \\
78 \\
78 \\
78 \\
78\end{array}$ & $\begin{array}{l}75 . \\
71 . \\
74^{\circ} \\
74^{\circ} \\
72 .\end{array}$ & $\begin{array}{l}41 . \\
45 . \\
49 \\
45 . \\
35 .\end{array}$ & $\begin{array}{l}48 \\
52 \\
54 \\
48 \\
44 .\end{array}$ & $\begin{array}{l}48 \\
49 \\
52 \\
48 \\
40\end{array}$ \\
\hline $\begin{array}{l}182 \\
183 \\
184 \\
185 \\
186\end{array}$ & $\begin{array}{l}7 \\
7 \\
7 \\
7 \\
7\end{array}$ & $\begin{array}{l}1 \\
2 \\
3 \\
4 \\
5\end{array}$ & $\begin{array}{l}78 \\
78 \\
78 \\
78 \\
78\end{array}$ & $\begin{array}{l}75 . \\
78 . \\
79 . \\
77 . \\
16 .\end{array}$ & $\begin{array}{l}38 . \\
33 . \\
34 . \\
30 \\
30\end{array}$ & $\begin{array}{l}47 \\
43 \\
45 \\
51 \\
45\end{array}$ & $\begin{array}{l}45 \\
41 \\
40 \\
45 \\
42\end{array}$ \\
\hline $\begin{array}{l}187 \\
188 \\
189 \\
190 \\
191\end{array}$ & $\begin{array}{l}7 \\
7 \\
7 \\
7 \\
7\end{array}$ & $\begin{array}{r}6 \\
7 \\
8 \\
9 \\
10\end{array}$ & $\begin{array}{l}78 \\
78 \\
78 \\
78 \\
78\end{array}$ & $\begin{array}{l}74 . \\
19 . \\
77 . \\
75 . \\
70 .\end{array}$ & $\begin{array}{l}36 \\
37 \\
45 \\
43 \\
45\end{array}$ & $\begin{array}{l}46 . \\
60 . \\
62 . \\
50 . \\
54 .\end{array}$ & $\begin{array}{l}44 . \\
48 . \\
51 . \\
46 . \\
50\end{array}$ \\
\hline
\end{tabular}


May-September 1977, 1978, and 1979--Continued

\begin{tabular}{|c|c|c|c|c|c|c|c|}
\hline $\begin{array}{l}\text { PAN } \\
\text { MAX } \\
\text { (F) }\end{array}$ & $\begin{array}{l}\text { WATER } \\
\text { MIN } \\
\text { (F) }\end{array}$ & $\begin{array}{l}\text { PRECIP } \\
\left(I N_{0}\right)\end{array}$ & $\begin{array}{l}\text { WIND } \\
\text { (MI./DAY) }\end{array}$ & $\begin{array}{l}\text { PAN } \\
\text { EVAP } \\
\text { (IN.) }\end{array}$ & $\begin{array}{l}\text { DAII } \\
\text { TEMP } \\
\text { (F) }\end{array}$ & $\begin{array}{l}\text { AVG } \\
\text { REL HUM } \\
\text { (PERCENT) }\end{array}$ & $\begin{array}{l}\text { SOLAR } \\
\text { RAD } \\
\text { (LY.) }\end{array}$ \\
\hline $\begin{array}{l}75 . \\
73 . \\
73 \\
77 \\
75 .\end{array}$ & $\begin{array}{l}41 . \\
39 \\
43 \\
37 \\
39\end{array}$ & $\begin{array}{l}0.00 \\
0.00 \\
0.00 \\
0.00 \\
0.00\end{array}$ & $\begin{array}{l}1150^{\circ} \\
1200^{\circ} \\
1430^{\circ} \\
1040^{\circ}\end{array}$ & $\begin{array}{l}.48 \\
.41 \\
.50 \\
.39 \\
.45\end{array}$ & $\begin{array}{l}61 . \\
56 . \\
53 . \\
57 . \\
53 .\end{array}$ & $\begin{array}{l}18 . \\
21 . \\
50 . \\
39 . \\
40 .\end{array}$ & $\begin{array}{l}588^{\circ} \\
554 . \\
415 . \\
569 . \\
626 .\end{array}$ \\
\hline $\begin{array}{l}77 . \\
75 . \\
82 . \\
50 \\
73 .\end{array}$ & $\begin{array}{l}45 . \\
37 \\
45 \\
50 \\
41\end{array}$ & $\begin{array}{l}0.00 \\
0.00 \\
0.00 \\
0.00 \\
0.00\end{array}$ & $\begin{array}{r}944^{\circ} \\
77^{\circ} \\
177^{\circ} \\
178^{\circ} \\
97^{\circ}\end{array}$ & $\begin{array}{l}.40 \\
.49 \\
.55 \\
.49 \\
.41\end{array}$ & $\begin{array}{l}57 . \\
58 \\
62 . \\
65 \\
64\end{array}$ & $\begin{array}{l}48 . \\
29 . \\
31 . \\
32 . \\
33 .\end{array}$ & $\begin{array}{l}588^{\circ} \\
492^{\circ} \\
593^{\circ} \\
554^{\circ} \\
605^{\circ}\end{array}$ \\
\hline $\begin{array}{l}75 . \\
73 . \\
75 \\
72 . \\
77 .\end{array}$ & $\begin{array}{l}41 \\
48 \\
48 \\
46 \\
45\end{array}$ & $\begin{array}{r}0.00 \\
0.00 \\
.04 \\
.08 \\
0.00\end{array}$ & $\begin{array}{l}92 . \\
69 . \\
77 . \\
63 . \\
39 .\end{array}$ & $\begin{array}{l}.33 \\
.19 \\
.25 \\
.22 \\
.37\end{array}$ & $\begin{array}{l}55 . \\
56 . \\
58 . \\
56 . \\
56 .\end{array}$ & $\begin{array}{l}35 . \\
60 . \\
65 . \\
79 . \\
64 .\end{array}$ & $\begin{array}{l}588^{\circ} \\
429 \\
310^{\circ} \\
317^{\circ} \\
482\end{array}$ \\
\hline $\begin{array}{l}81 . \\
79 . \\
750 \\
77 . \\
75 .\end{array}$ & $\begin{array}{l}41 . \\
43 \\
41 \\
41 \\
41\end{array}$ & $\begin{array}{l}0.00 \\
0.00 \\
0.00 \\
0.00 \\
0.00\end{array}$ & $\begin{array}{r}67 . \\
98 . \\
102 . \\
81 . \\
100 .\end{array}$ & $\begin{array}{l}.43 \\
.47 \\
.48 \\
.48 \\
.42\end{array}$ & $\begin{array}{l}56 . \\
59 . \\
59 . \\
56 . \\
55 .\end{array}$ & $\begin{array}{l}54 . \\
390^{\circ} \\
320^{\circ} \\
27 \\
28 .\end{array}$ & $\begin{array}{l}468 . \\
501 . \\
573 . \\
608 . \\
608 .\end{array}$ \\
\hline $\begin{array}{l}79 . \\
79 . \\
77 . \\
72 . \\
64 .\end{array}$ & $\begin{array}{l}410^{\circ} \\
450^{\circ} \\
460^{\circ} \\
46 .\end{array}$ & $\begin{array}{l}0.00 \\
0.00 \\
0.00 \\
0.00 \\
.00\end{array}$ & $\begin{array}{r}94 . \\
111 . \\
105 . \\
76 . \\
79 .\end{array}$ & $\begin{array}{l}.04 \\
.48 \\
.38 \\
.15 \\
.27\end{array}$ & $\begin{array}{l}55 . \\
59 . \\
63 . \\
58 . \\
54 .\end{array}$ & $\begin{array}{l}33 . \\
55^{\circ} \\
39 . \\
46 . \\
73 .\end{array}$ & $\begin{array}{l}573 \\
608^{\circ} \\
530 \\
346^{\circ} \\
226 .\end{array}$ \\
\hline
\end{tabular}


Table 6.--Climatic data, Antero Reservoir station,

\begin{tabular}{|c|c|c|c|c|c|c|c|}
\hline $\begin{array}{r}\text { DAY } \\
\text { OF } \\
\text { YEAR }\end{array}$ & MONTH & DAY & YEAR & $\begin{array}{l}\text { IEMF } \\
\text { MAX } \\
\text { (F) }\end{array}$ & $\begin{array}{l}\text { TURE } \\
\text { MIN } \\
\text { (F) }\end{array}$ & $\begin{array}{l}\text { DRY } \\
\text { BULB } \\
\text { (F) }\end{array}$ & $\begin{array}{l}\text { WET } \\
\text { BULB } \\
(F)\end{array}$ \\
\hline $\begin{array}{l}192 \\
193 \\
194 \\
195 \\
196\end{array}$ & $\begin{array}{l}7 \\
7 \\
7 \\
7 \\
7\end{array}$ & $\begin{array}{l}11 \\
12 \\
13 \\
14 \\
15\end{array}$ & $\begin{array}{l}78 \\
78 \\
78 \\
78 \\
78\end{array}$ & $\begin{array}{l}74{ }^{\circ} \\
78 . \\
78 . \\
83{ }^{\circ} \\
19 .\end{array}$ & $\begin{array}{l}38 \\
41 . \\
40 \\
41 . \\
39 .\end{array}$ & $\begin{array}{l}51 . \\
51 . \\
52 . \\
52 . \\
48 .\end{array}$ & $\begin{array}{l}48 \\
46 \\
49 \\
47 \\
45\end{array}$ \\
\hline $\begin{array}{l}197 \\
198 \\
199 \\
200 \\
201\end{array}$ & $\begin{array}{l}7 \\
7 \\
7 \\
7 \\
7\end{array}$ & $\begin{array}{l}16 \\
17 \\
18 \\
19 \\
20\end{array}$ & $\begin{array}{l}78 \\
78 \\
78 \\
78 \\
78\end{array}$ & $\begin{array}{l}17 . \\
77 . \\
16 . \\
79 . \\
76 .\end{array}$ & $\begin{array}{l}410^{\circ} \\
420^{\circ} \\
39{ }^{\circ} \\
37{ }^{\circ}\end{array}$ & $\begin{array}{l}53 . \\
51 . \\
51 . \\
48 . \\
56 .\end{array}$ & $\begin{array}{l}50 . \\
49 . \\
46 . \\
45 . \\
51 .\end{array}$ \\
\hline $\begin{array}{l}202 \\
203 \\
204 \\
205 \\
206\end{array}$ & $\begin{array}{l}7 \\
7 \\
7 \\
7 \\
7\end{array}$ & $\begin{array}{l}21 \\
22 \\
23 \\
24 \\
25\end{array}$ & $\begin{array}{l}78 \\
78 \\
78 \\
78 \\
78\end{array}$ & $\begin{array}{l}76 . \\
76 . \\
18 . \\
80 . \\
82 .\end{array}$ & $\begin{array}{l}40^{\circ} \\
400^{\circ} \\
360^{\circ} \\
39^{\circ}\end{array}$ & $\begin{array}{l}550^{\circ} \\
48 . \\
470^{\circ} \\
510^{\circ}\end{array}$ & $\begin{array}{l}51 \\
470^{\circ} \\
450^{\circ} \\
46\end{array}$ \\
\hline $\begin{array}{l}207 \\
208 \\
209 \\
210 \\
211\end{array}$ & $\begin{array}{l}7 \\
7 \\
7 \\
7 \\
7\end{array}$ & $\begin{array}{l}26 \\
27 \\
28 \\
29 \\
30\end{array}$ & $\begin{array}{l}78 \\
78 \\
78 \\
78 \\
78\end{array}$ & $\begin{array}{l}81 . \\
83 . \\
81 . \\
19 . \\
18 .\end{array}$ & $\begin{array}{l}38 \\
41 . \\
39 \\
40 \\
48\end{array}$ & $\begin{array}{l}50 . \\
51 . \\
53 . \\
50 . \\
50 .\end{array}$ & $\begin{array}{l}47 \\
46 \\
49 \\
48 \\
48\end{array}$ \\
\hline $\begin{array}{l}212 \\
213 \\
214 \\
215 \\
216\end{array}$ & $\begin{array}{l}7 \\
8 \\
8 \\
8 \\
8\end{array}$ & $\begin{array}{r}31 \\
1 \\
2 \\
3 \\
4\end{array}$ & $\begin{array}{l}78 \\
78 \\
78 \\
78 \\
78\end{array}$ & $\begin{array}{l}78 . \\
18 . \\
77 . \\
68 \\
68 .\end{array}$ & $\begin{array}{l}35 . \\
35 \\
41 \\
41 . \\
34\end{array}$ & $\begin{array}{l}490^{\circ} \\
430^{\circ} \\
480^{\circ} \\
410^{\circ}\end{array}$ & $\begin{array}{l}46 . \\
420^{\circ} \\
470^{\circ} \\
46 .\end{array}$ \\
\hline
\end{tabular}


May-September 1977, 1978, and 1979--Continued

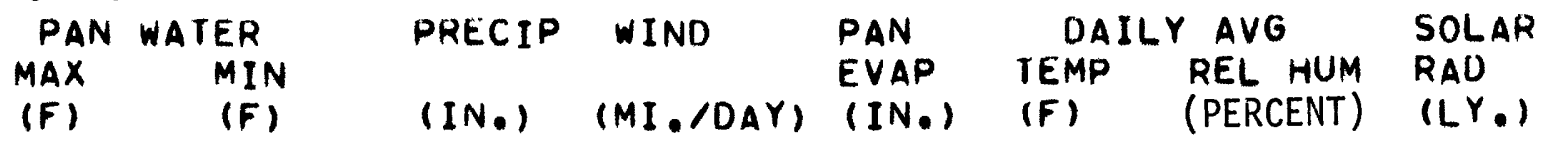

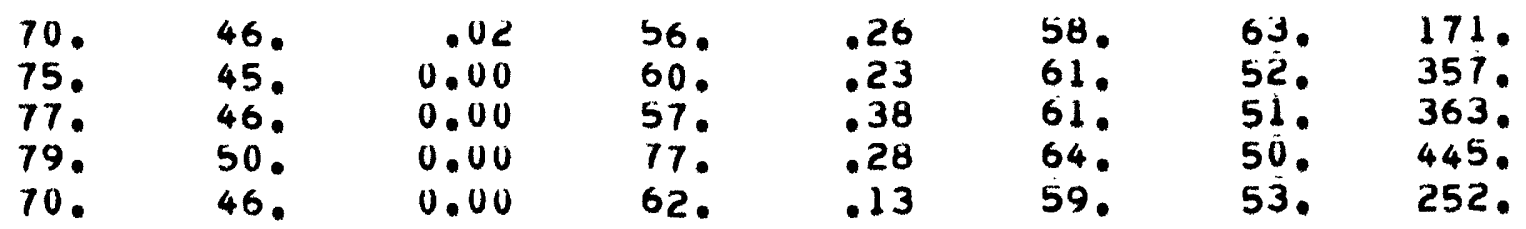

\begin{tabular}{|c|c|c|c|c|c|c|}
\hline $\begin{array}{l}70 . \\
73 . \\
77 . \\
77 . \\
75 .\end{array}$ & $\begin{array}{l}46 . \\
48 \\
48 \\
46 \\
46\end{array}$ & $\begin{array}{l}.01 \\
.05 \\
0.00 \\
0.00 \\
0.00\end{array}$ & $\begin{array}{r}69 . \\
63 . \\
52, \\
104 . \\
124 .\end{array}$ & $\begin{array}{r}.34 \\
.30 \\
.32 \\
.29 \\
.42\end{array}$ & $\begin{array}{l}57 . \\
61 \text {. } \\
60 . \\
59 . \\
57 .\end{array}$ & $\begin{array}{l}72 . \\
63 . \\
56 . \\
49 . \\
64 .\end{array}$ \\
\hline
\end{tabular}

\begin{tabular}{|c|c|c|c|c|c|c|}
\hline $\begin{array}{l}75 \\
86 . \\
84 . \\
82 . \\
81\end{array}$ & $\begin{array}{l}48 . \\
46 . \\
46 . \\
46\end{array}$ & $\begin{array}{l}0.00 \\
.01 \\
0.00 \\
0.00 \\
0.00\end{array}$ & $\begin{array}{l}74 . \\
640^{\circ} \\
620^{\circ} \\
45^{\circ}\end{array}$ & $\begin{array}{l}.36 \\
.32 \\
.39 \\
.25 \\
.49\end{array}$ & $\begin{array}{l}61 . \\
56 . \\
57 . \\
58 . \\
63 .\end{array}$ & $\begin{array}{l}61 . \\
63 . \\
63 . \\
53 \\
39 .\end{array}$ \\
\hline
\end{tabular}

\begin{tabular}{|c|c|c|c|c|c|c|}
\hline $\begin{array}{l}75 . \\
86 . \\
73 . \\
75 . \\
82 .\end{array}$ & $\begin{array}{l}45 . \\
46 . \\
46 . \\
46\end{array}$ & $\begin{array}{r}0.00 \\
0.00 \\
0.00 \\
0.00 \\
.01\end{array}$ & $\begin{array}{l}86 . \\
85 . \\
82 . \\
68 . \\
51 .\end{array}$ & $\begin{array}{r}.35 \\
.34 \\
.31 \\
.37 \\
.21\end{array}$ & $\begin{array}{l}60 . \\
61: \\
61 . \\
58 . \\
59 .\end{array}$ & $\begin{array}{l}42 . \\
56 . \\
51 . \\
61 . \\
66 .\end{array}$ \\
\hline
\end{tabular}

\begin{tabular}{|c|c|c|c|c|c|c|}
\hline $\begin{array}{l}73 . \\
70 . \\
77 . \\
79 . \\
72 .\end{array}$ & $\begin{array}{l}46 . \\
43 . \\
45 . \\
46 . \\
43 .\end{array}$ & $\begin{array}{r}.02 \\
.12 \\
0.00 \\
.37 \\
.01\end{array}$ & $\begin{array}{l}93 . \\
69 . \\
55 . \\
54 . \\
50 .\end{array}$ & $\begin{array}{l}.27 \\
.29 \\
.32 \\
.25 \\
.34\end{array}$ & $\begin{array}{l}66 . \\
53 . \\
53 . \\
48 . \\
51 .\end{array}$ & $\begin{array}{l}68 . \\
66 \\
67 \\
87 \\
68\end{array}$ \\
\hline
\end{tabular}


Table 6.--Climatic data, Antero Reservoir station,

\begin{tabular}{|c|c|c|c|c|c|c|c|}
\hline $\begin{array}{r}\text { DAY } \\
\text { OF } \\
\text { YEAR }\end{array}$ & MONTH & DAY & YEAR & $\begin{array}{l}\text { TEMF } \\
\text { MAX } \\
\text { (F) }\end{array}$ & $\begin{array}{l}\text { TURE } \\
\text { MIN } \\
\text { (F) }\end{array}$ & $\begin{array}{l}\text { DRY } \\
\text { BULB } \\
\text { (F) }\end{array}$ & $\begin{array}{l}\text { WET } \\
\text { BULB } \\
\text { (F) }\end{array}$ \\
\hline $\begin{array}{l}217 \\
218 \\
219 \\
220 \\
221\end{array}$ & $\begin{array}{l}8 \\
8 \\
8 \\
8 \\
8\end{array}$ & $\begin{array}{l}5 \\
6 \\
7 \\
8 \\
9\end{array}$ & $\begin{array}{l}78 \\
78 \\
78 \\
78 \\
78\end{array}$ & $\begin{array}{l}76 . \\
79 . \\
73 . \\
72 . \\
71 .\end{array}$ & $\begin{array}{l}37 . \\
40 . \\
350^{\circ} \\
37 . \\
37 .\end{array}$ & $\begin{array}{l}48 \\
48 \\
45 \\
47 \\
44 \circ\end{array}$ & $\begin{array}{l}47 . \\
47 \circ^{\circ} \\
44 \circ^{\circ} \\
45^{\circ} \\
40^{\circ}\end{array}$ \\
\hline $\begin{array}{l}222 \\
223 \\
224 \\
225 \\
226\end{array}$ & $\begin{array}{l}8 \\
8 \\
8 \\
8 \\
8\end{array}$ & $\begin{array}{l}10 \\
11 \\
12 \\
13 \\
14\end{array}$ & $\begin{array}{l}78 \\
78 \\
78 \\
78 \\
78\end{array}$ & $\begin{array}{l}14 . \\
16 . \\
77 . \\
12 . \\
67 .\end{array}$ & $\begin{array}{l}36 . \\
38 . \\
39 . \\
36 . \\
32 .\end{array}$ & $\begin{array}{l}45 . \\
49 . \\
50 \\
46 . \\
41 .\end{array}$ & $\begin{array}{l}420^{\circ} \\
480^{\circ} \\
490^{\circ} \\
320^{\circ}\end{array}$ \\
\hline $\begin{array}{l}227 \\
228 \\
229 \\
230 \\
231\end{array}$ & $\begin{array}{l}8 \\
8 \\
8 \\
8 \\
8\end{array}$ & $\begin{array}{l}15 \\
16 \\
17 \\
18 \\
19\end{array}$ & $\begin{array}{l}78 \\
78 \\
78 \\
78 \\
78\end{array}$ & $\begin{array}{l}72 . \\
74 . \\
12 . \\
64 . \\
12 .\end{array}$ & $\begin{array}{l}36 . \\
49 . \\
37 . \\
29 . \\
36 .\end{array}$ & $\begin{array}{l}53 \\
60 \\
40 \\
39 \\
42\end{array}$ & $\begin{array}{l}47 . \\
50 . \\
39 . \\
37 . \\
42 .\end{array}$ \\
\hline $\begin{array}{l}232 \\
233 \\
234 \\
235 \\
236\end{array}$ & $\begin{array}{l}8 \\
8 \\
8 \\
8 \\
8\end{array}$ & $\begin{array}{l}20 \\
21 \\
22 \\
23 \\
24\end{array}$ & $\begin{array}{l}78 \\
78 \\
78 \\
78 \\
78\end{array}$ & $\begin{array}{l}75 . \\
77 . \\
68 . \\
75 . \\
73 .\end{array}$ & $\begin{array}{l}37 . \\
36 . \\
36 . \\
38 . \\
42 .\end{array}$ & $\begin{array}{l}46 . \\
52 . \\
46 . \\
45 . \\
51 .\end{array}$ & $\begin{array}{l}45 \\
46 \\
45 \\
41 \\
47\end{array}$ \\
\hline $\begin{array}{l}237 \\
238 \\
239 \\
240 \\
241\end{array}$ & $\begin{array}{l}8 \\
8 \\
8 \\
8 \\
8\end{array}$ & $\begin{array}{l}25 \\
26 \\
27 \\
28 \\
29\end{array}$ & $\begin{array}{l}78 \\
78 \\
78 \\
78 \\
78\end{array}$ & $\begin{array}{l}70 . \\
13 . \\
15 . \\
72 . \\
67 .\end{array}$ & $\begin{array}{l}34 . \\
36 . \\
28 \\
38 . \\
35 .\end{array}$ & $\begin{array}{l}41 . \\
470^{\circ} \\
43 . \\
440^{\circ} \\
41 .\end{array}$ & $\begin{array}{l}39 \\
45 \\
40 \\
44{ }^{\circ} \\
40\end{array}$ \\
\hline
\end{tabular}




\begin{tabular}{|c|c|c|c|c|c|c|c|}
\hline $\begin{array}{l}\text { May-Sez } \\
\text { PAN } \\
\text { MAX } \\
\text { (F) }\end{array}$ & $\begin{array}{l}\text { tember } 1977, \\
\text { WATER } \\
\text { MIN } \\
\text { (F) }\end{array}$ & $\begin{array}{l}\text { 1978, an } \\
\text { PRECIP } \\
(\text { IN.) }\end{array}$ & $\begin{array}{l}\text { 1979--Con } \\
\text { WIND } \\
\text { (MI./DAY) }\end{array}$ & $\begin{array}{l}\text { inued } \\
\text { PAN } \\
\text { EVAP } \\
\text { (IN.) }\end{array}$ & $\begin{array}{l}\text { DAILY } \\
\text { TEMP } \\
\text { (F) }\end{array}$ & $\begin{array}{l}\text { AVG } \\
\text { REL HUM } \\
\text { (PERCENT) }\end{array}$ & $\begin{array}{l}\text { SOLAR } \\
\text { RAD } \\
(L Y .)\end{array}$ \\
\hline $\begin{array}{l}81 . \\
73 \\
73 \\
72 \\
72\end{array}$ & $\begin{array}{l}43 . \\
45 . \\
41 . \\
43 . \\
43 .\end{array}$ & $\begin{array}{r}0.00 \\
.02 \\
0.00 \\
.02 \\
.05\end{array}$ & $\begin{array}{l}58 . \\
83 . \\
71 . \\
73 . \\
63 .\end{array}$ & $\begin{array}{r}.22 \\
.18 \\
.23 \\
.32 \\
.22\end{array}$ & $\begin{array}{l}53 . \\
54 . \\
54 . \\
53 . \\
53 .\end{array}$ & $\begin{array}{l}54 . \\
61 . \\
58 \\
60 \\
71 .\end{array}$ & $\begin{array}{l}645 . \\
412 . \\
494 . \\
471 \\
* 40\end{array}$ \\
\hline $\begin{array}{l}68 . \\
70 . \\
70 \\
73 . \\
72 .\end{array}$ & $\begin{array}{l}45 . \\
45 . \\
46 . \\
41 . \\
37 .\end{array}$ & $\begin{array}{r}0.00 \\
.05 \\
.20 \\
.01 \\
.04\end{array}$ & $\begin{array}{r}57 . \\
72 . \\
75 . \\
159 . \\
67 .\end{array}$ & $\begin{array}{l}.20 \\
.24 \\
.24 \\
.40 \\
.29\end{array}$ & $\begin{array}{l}53 . \\
54 . \\
56 . \\
54 . \\
49 .\end{array}$ & $\begin{array}{l}67 . \\
67 . \\
66 . \\
70 . \\
67 .\end{array}$ & $\begin{array}{l}340^{\circ} \\
363^{\circ} \\
392^{\circ} \\
451^{\circ} \\
562^{\circ}\end{array}$ \\
\hline $\begin{array}{l}75 . \\
75 . \\
72 . \\
66 . \\
73 .\end{array}$ & $\begin{array}{l}37 . \\
43 . \\
43 . \\
37 . \\
37 .\end{array}$ & $\begin{array}{l}0.00 \\
0.00 \\
0.00 \\
0.00 \\
0.00\end{array}$ & $\begin{array}{l}170 \\
170^{\circ} \\
146^{\circ} \\
85^{\circ} \\
56 .\end{array}$ & $\begin{array}{r}.48 \\
.46 \\
.51 \\
.29 \\
.31\end{array}$ & $\begin{array}{l}51 . \\
56 . \\
60 . \\
50 . \\
49 .\end{array}$ & $\begin{array}{l}39 . \\
40 . \\
29 . \\
52 . \\
65 .\end{array}$ & $\begin{array}{l}612 . \\
593 . \\
603 . \\
533 . \\
566 .\end{array}$ \\
\hline $\begin{array}{l}77 . \\
77 . \\
70 \\
77 . \\
72 .\end{array}$ & $\begin{array}{l}41 . \\
45 \circ \\
41 . \\
43 . \\
45 .\end{array}$ & $\begin{array}{r}0.00 \\
.01 \\
0.00 \\
0.00 \\
0.00\end{array}$ & $\begin{array}{l}59 . \\
130 \\
69 \\
49 \\
65 .\end{array}$ & $\begin{array}{r}.23 \\
.29 \\
.24 \\
.23 \\
.21\end{array}$ & $\begin{array}{l}55 . \\
55 . \\
55 . \\
56 . \\
53 .\end{array}$ & $\begin{array}{l}58 . \\
55 . \\
71 . \\
59 . \\
51 .\end{array}$ & $\begin{array}{l}494^{\circ} \\
431^{\circ} \\
356^{\circ} \\
490^{\circ} \\
297^{\circ}\end{array}$ \\
\hline $\begin{array}{l}73 \\
75 \\
75 \\
70 \\
68 .\end{array}$ & $\begin{array}{l}45 . \\
43 \\
39 \\
39 \\
39 .\end{array}$ & $\begin{array}{r}0.00 \\
0.00 \\
0.00 \\
.05 \\
0.00\end{array}$ & $\begin{array}{l}41 . \\
690^{\circ} \\
82 . \\
72 . \\
55 .\end{array}$ & $\begin{array}{r}.26 \\
.38 \\
.22 \\
.22 \\
.24\end{array}$ & $\begin{array}{l}54 . \\
53 . \\
57 . \\
50 \\
47 .\end{array}$ & $\begin{array}{l}69 . \\
58 . \\
32 \\
63 . \\
75 .\end{array}$ & $\begin{array}{l}312 . \\
4360^{\circ} \\
524{ }^{\circ} \\
410^{\circ} \\
389^{\circ}\end{array}$ \\
\hline
\end{tabular}


Table 6.--Climatic data, Antero Reservoir station,

\begin{tabular}{|c|c|c|c|c|c|c|c|}
\hline $\begin{array}{l}\text { DAY } \\
\text { OF } \\
\text { YEAR }\end{array}$ & MONTH & DAY & YEAR & $\begin{array}{l}\text { TEMP } \\
\text { MAX } \\
\text { (F) }\end{array}$ & $\begin{array}{l}\text { TURE } \\
\text { MIN } \\
\text { (F) }\end{array}$ & $\begin{array}{l}\text { DRY } \\
\text { BULB } \\
\text { (F) }\end{array}$ & $\begin{array}{l}\text { WET } \\
\text { BULB } \\
\text { (F) }\end{array}$ \\
\hline $\begin{array}{l}242 \\
243 \\
244 \\
245 \\
246\end{array}$ & $\begin{array}{l}8 \\
8 \\
9 \\
9 \\
9\end{array}$ & $\begin{array}{r}30 \\
31 \\
1 \\
2 \\
3\end{array}$ & $\begin{array}{l}78 \\
78 \\
78 \\
78 \\
78\end{array}$ & $\begin{array}{l}10 . \\
12 . \\
12 . \\
11 . \\
16 .\end{array}$ & $\begin{array}{l}330^{\circ} \\
340^{\circ} \\
320^{\circ} \\
310^{\circ}\end{array}$ & $\begin{array}{l}40 . \\
430^{\circ} \\
420^{\circ} \\
370^{\circ}\end{array}$ & $\begin{array}{l}40 . \\
40 \\
39 . \\
36 . \\
42 .\end{array}$ \\
\hline $\begin{array}{l}247 \\
248 \\
249 \\
250 \\
251\end{array}$ & $\begin{array}{l}9 \\
9 \\
9 \\
9 \\
9\end{array}$ & $\begin{array}{l}4 \\
5 \\
6 \\
7 \\
8\end{array}$ & $\begin{array}{l}78 \\
78 \\
18 \\
78 \\
78\end{array}$ & $\begin{array}{l}19 . \\
810^{\circ} \\
830^{\circ} \\
16 .\end{array}$ & $\begin{array}{l}34 \\
35{ }^{\circ} \\
37 \\
36 \\
33\end{array}$ & $\begin{array}{l}420^{\circ} \\
420^{\circ} \\
410^{\circ} \\
420^{\circ}\end{array}$ & $\begin{array}{l}41 . \\
410 \\
410 \\
410 \\
38\end{array}$ \\
\hline $\begin{array}{l}252 \\
253 \\
254 \\
255 \\
256\end{array}$ & $\begin{array}{l}9 \\
9 \\
9 \\
9 \\
9\end{array}$ & $\begin{array}{l}9 \\
10 \\
11 \\
12 \\
13\end{array}$ & $\begin{array}{l}78 \\
78 \\
78 \\
78 \\
78\end{array}$ & $\begin{array}{l}15 . \\
10 . \\
60 . \\
61 . \\
69 .\end{array}$ & $\begin{array}{l}35 . \\
42 . \\
38 \\
23 \\
22 \\
\end{array}$ & $\begin{array}{l}45{ }^{\circ} \\
53{ }^{\circ} \\
45^{\circ} \\
33 \\
28\end{array}$ & $\begin{array}{l}44 . \\
470^{\circ} \\
410^{\circ} \\
30\end{array}$ \\
\hline $\begin{array}{l}257 \\
258 \\
259 \\
260 \\
261\end{array}$ & $\begin{array}{l}9 \\
9 \\
9 \\
9 \\
9\end{array}$ & $\begin{array}{l}14 \\
15 \\
16 \\
17 \\
18\end{array}$ & $\begin{array}{l}18 \\
78 \\
78 \\
78 \\
78\end{array}$ & $\begin{array}{l}68 . \\
12 . \\
11 . \\
62 . \\
63 .\end{array}$ & $\begin{array}{l}28{ }^{\circ} \\
42{ }^{\circ} \\
430^{\circ} \\
40 \\
30^{\circ}\end{array}$ & $\begin{array}{l}42 . \\
520^{\circ} \\
470^{\circ} \\
490^{\circ}\end{array}$ & $\begin{array}{l}39 . \\
480^{\circ} \\
46{ }^{\circ} \\
46 \\
35\end{array}$ \\
\hline $\begin{array}{l}262 \\
263 \\
264 \\
265 \\
266\end{array}$ & $\begin{array}{l}9 \\
9 \\
9 \\
9 \\
9\end{array}$ & $\begin{array}{l}19 \\
20 \\
21 \\
22 \\
23\end{array}$ & $\begin{array}{l}78 \\
78 \\
78 \\
78 \\
78\end{array}$ & $\begin{array}{l}45 . \\
43 \\
60 \\
66 \\
71 .\end{array}$ & $\begin{array}{l}28, \\
200^{\circ} \\
190^{\circ} \\
24{ }^{\circ}\end{array}$ & $\begin{array}{l}32 . \\
22{ }^{\circ} \\
28 \\
30 \\
36\end{array}$ & $\begin{array}{l}32{ }^{\circ} \\
22{ }^{\circ} \\
27 \\
30 \\
36\end{array}$ \\
\hline $\begin{array}{l}267 \\
268 \\
269 \\
270 \\
271\end{array}$ & $\begin{array}{l}9 \\
9 \\
9 \\
9 \\
9\end{array}$ & $\begin{array}{l}24 \\
25 \\
26 \\
27 \\
28\end{array}$ & $\begin{array}{l}78 \\
78 \\
78 \\
78 \\
78\end{array}$ & $\begin{array}{l}62 . \\
61 . \\
10 . \\
69 . \\
10 .\end{array}$ & $\begin{array}{l}410^{\circ} \\
25{ }^{\circ} \\
25{ }^{\circ} \\
26 \\
\text {. }\end{array}$ & $\begin{array}{l}430^{\circ} \\
30 \\
30 \\
310^{\circ} \\
37\end{array}$ & $\begin{array}{l}410^{\circ} \\
300^{\circ} \\
30 \\
310^{\circ}\end{array}$ \\
\hline $\begin{array}{l}272 \\
273\end{array}$ & $\begin{array}{l}9 \\
9\end{array}$ & $\begin{array}{l}29 \\
30\end{array}$ & $\begin{array}{l}78 \\
78\end{array}$ & $\begin{array}{l}70 . \\
72 .\end{array}$ & $\begin{array}{l}22 \\
25\end{array}$ & $\begin{array}{l}27 \\
30\end{array}$ & $\begin{array}{l}22 . \\
30\end{array}$ \\
\hline
\end{tabular}


May-September 1977, 1978, and 1979--Continued

\begin{tabular}{|c|c|c|c|c|c|c|c|}
\hline $\begin{array}{l}\text { PAN } \\
\text { MAX } \\
\text { (F) }\end{array}$ & $\begin{array}{l}\text { WATER } \\
\text { MIN } \\
\text { (F) }\end{array}$ & $\begin{array}{l}\text { PRECIP } \\
(I N \cdot)\end{array}$ & $\begin{array}{l}\text { WIND } \\
\text { (MI./DAY) }\end{array}$ & $\begin{array}{l}\text { PAN } \\
\text { EVAP } \\
\text { (IN.) }\end{array}$ & $\begin{array}{l}\text { DAI } \\
\text { TEMP } \\
\text { (F) }\end{array}$ & $\begin{array}{l}\text { Y AVG } \\
\text { REL HUM } \\
\text { (PERCENT) }\end{array}$ & $\begin{array}{l}\text { SOLAR } \\
\text { RAD } \\
(L Y .)\end{array}$ \\
\hline $\begin{array}{l}68 . \\
70 . \\
70 . \\
70 . \\
75 .\end{array}$ & $\begin{array}{l}39 . \\
41 . \\
41 . \\
39 \\
39\end{array}$ & $\begin{array}{l}0.00 \\
0.00 \\
0.00 \\
0.00 \\
0.00\end{array}$ & $\begin{array}{r}99 . \\
* 4 \\
65 . \\
53 . \\
54 .\end{array}$ & $\begin{array}{l}.23 \\
.00 \\
.28 \\
.26 \\
.27\end{array}$ & $\begin{array}{l}50 . \\
49 . \\
54 . \\
52 . \\
54 .\end{array}$ & $\begin{array}{l}63 . \\
62 . \\
54 . \\
59 . \\
58 .\end{array}$ & $\begin{array}{l}393 . \\
419^{\circ} \\
341 . \\
392 . \\
491 .\end{array}$ \\
\hline $\begin{array}{l}79 . \\
81 . \\
81 . \\
77 . \\
77 .\end{array}$ & $\begin{array}{l}43 \\
43 \\
43 \\
41 \\
41 \\
\end{array}$ & $\begin{array}{r}0.00 \\
0.00 \\
0.00 \\
.15 \\
0.00\end{array}$ & $\begin{array}{l}43 . \\
55 . \\
61 . \\
39 . \\
65 .\end{array}$ & $\begin{array}{r}.33 \\
.34 \\
.25 \\
.19 \\
.35\end{array}$ & $\begin{array}{l}51 . \\
58 . \\
58 . \\
52 . \\
53 .\end{array}$ & $\begin{array}{l}50 . \\
41 . \\
48 . \\
69 . \\
57 .\end{array}$ & $\begin{array}{l}5311^{\circ} \\
5210^{\circ} \\
480^{\circ} \\
4030^{\circ}\end{array}$ \\
\hline $\begin{array}{l}75 . \\
72 . \\
66 . \\
61 . \\
61 .\end{array}$ & $\begin{array}{l}41 . \\
41 . \\
39 \\
36 \\
36\end{array}$ & $\begin{array}{l}0.00 \\
0.00 \\
0.00 \\
0.00 \\
0.00\end{array}$ & $\begin{array}{l}127 . \\
1920^{\circ} \\
160 \circ \\
108^{\circ} \\
76 .\end{array}$ & $\begin{array}{r}.27 \\
.39 \\
.38 \\
.31 \\
.28\end{array}$ & $\begin{array}{l}54 . \\
54 . \\
52 . \\
46 . \\
40\end{array}$ & $\begin{array}{l}55 . \\
590^{\circ} \\
47 . \\
43 . \\
55 .\end{array}$ & $\begin{array}{l}469^{\circ} \\
393^{\circ} \\
490^{\circ} \\
508^{\circ}\end{array}$ \\
\hline $\begin{array}{l}70 . \\
72 . \\
70 . \\
66 . \\
61 .\end{array}$ & $\begin{array}{l}36 \\
41 . \\
43 \\
39 \\
36 \\
\end{array}$ & $\begin{array}{l}0.00 \\
0.00 \\
0.00 \\
0.00 \\
0.00\end{array}$ & $\begin{array}{l}108 . \\
105^{\circ} \\
2100^{\circ} \\
1970^{\circ}\end{array}$ & $\begin{array}{l}.26 \\
.35 \\
.29 \\
.32 \\
.00\end{array}$ & $\begin{array}{r}47 . \\
53 . \\
* * 4 * \\
* * * *\end{array}$ & $\begin{array}{r}47 . \\
520 \\
* 4 * 4 \\
* * 4 *\end{array}$ & $\begin{array}{l}4755^{\circ} \\
475^{\circ} \\
363^{\circ} \\
403 \\
369^{\circ}\end{array}$ \\
\hline $\begin{array}{l}46 . \\
54 . \\
66 . \\
70 . \\
72 .\end{array}$ & $\begin{array}{l}34 \\
32 \\
32 \\
34 \\
36\end{array}$ & $\begin{array}{l}.15 \\
0.00 \\
0.00 \\
0.00 \\
0.00\end{array}$ & $\begin{array}{l}52 . \\
52 . \\
40 . \\
13 . \\
100 .\end{array}$ & $\begin{array}{l}.07 \\
.20 \\
.03 \\
.44 \\
.10\end{array}$ & $\begin{array}{l}33 \\
29 \\
32 \\
40 \\
47\end{array}$ & $\begin{array}{l}75 . \\
88 . \\
61 . \\
56 . \\
52 .\end{array}$ & $\begin{array}{l}105 . \\
190 \\
408 \\
423 . \\
433 .\end{array}$ \\
\hline $\begin{array}{l}54 . \\
66 . \\
70 \\
66 . \\
68 .\end{array}$ & $\begin{array}{l}39 \\
37 \\
37 \\
36 \\
37\end{array}$ & $\begin{array}{l}0.00 \\
0.00 \\
0.00 \\
0.00 \\
0.00\end{array}$ & $\begin{array}{l}41 . \\
52 . \\
59 . \\
65 . \\
71 .\end{array}$ & $\begin{array}{l}.18 \\
.24 \\
.13 \\
.33 \\
.28\end{array}$ & $\begin{array}{l}43 \\
45 \\
46 . \\
45 \\
47\end{array}$ & $\begin{array}{l}61 . \\
80 . \\
56 . \\
51 . \\
52 .\end{array}$ & $\begin{array}{l}151 . \\
360 \\
392 \\
343 \\
372\end{array}$ \\
\hline $\begin{array}{l}68 . \\
70 .\end{array}$ & $\begin{array}{l}36 . \\
36 .\end{array}$ & $\begin{array}{l}0.00 \\
0.00\end{array}$ & $\begin{array}{l}45 . \\
89 .\end{array}$ & $\begin{array}{l}.29 \\
.16\end{array}$ & $\begin{array}{l}49 . \\
44 .\end{array}$ & $\begin{array}{l}41 . \\
54 .\end{array}$ & $\begin{array}{l}410^{\circ} \\
285^{\circ}\end{array}$ \\
\hline
\end{tabular}


Table 6.--Climatic data, Antero Reservoir station,

\begin{tabular}{|c|c|c|c|c|c|c|}
\hline $\begin{array}{r}\text { OAY } \\
\text { OF } \\
\text { YEAR }\end{array}$ & MONTH & DAY & YEAR & $\begin{array}{l}\text { TEMF } \\
\text { MAX } \\
\text { (F) }\end{array}$ & $\begin{array}{l}\text { URE } \\
\text { MIN } \\
\text { (F) }\end{array}$ & $\begin{array}{l}\text { DRY } \\
\text { BULB } \\
(F)\end{array}$ \\
\hline $\begin{array}{l}127 \\
128 \\
129 \\
130 \\
131\end{array}$ & $\begin{array}{l}5 \\
5 \\
5 \\
5 \\
5\end{array}$ & $\begin{array}{r}7 \\
8 \\
9 \\
10 \\
11\end{array}$ & $\begin{array}{l}79 \\
79 \\
79 \\
79 \\
79\end{array}$ & 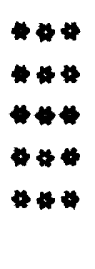 & 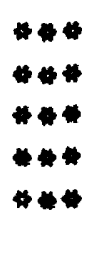 & $\begin{array}{l}* * * \\
* * 4 \\
* * 4 \\
* * 4 \\
* 4 *\end{array}$ \\
\hline $\begin{array}{l}132 \\
133 \\
134 \\
135 \\
136\end{array}$ & $\begin{array}{l}5 \\
5 \\
5 \\
5 \\
5\end{array}$ & $\begin{array}{l}12 \\
13 \\
14 \\
15 \\
16\end{array}$ & $\begin{array}{l}79 \\
79 \\
79 \\
79 \\
79\end{array}$ & 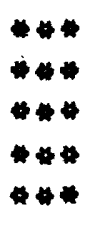 & 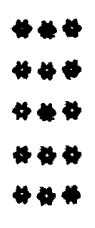 & 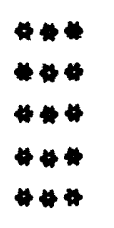 \\
\hline $\begin{array}{l}137 \\
138 \\
139 \\
140 \\
141\end{array}$ & $\begin{array}{l}5 \\
5 \\
5 \\
5 \\
5\end{array}$ & $\begin{array}{l}17 \\
18 \\
19 \\
20 \\
21\end{array}$ & $\begin{array}{l}79 \\
79 \\
19 \\
79 \\
79\end{array}$ & $\begin{array}{l}* \# * \\
* \# \% \\
65 . \\
64 . \\
46 .\end{array}$ & $\begin{array}{l}* * 4 \\
* * \\
29 . \\
34 . \\
32 .\end{array}$ & $\begin{array}{l}40 \\
40 \\
40 \\
44 . \\
36 .\end{array}$ \\
\hline $\begin{array}{l}142 \\
143 \\
144 \\
145 \\
146\end{array}$ & $\begin{array}{l}5 \\
5 \\
5 \\
5 \\
5\end{array}$ & $\begin{array}{l}22 \\
23 \\
24 \\
25 \\
26\end{array}$ & $\begin{array}{l}79 \\
79 \\
79 \\
79 \\
19\end{array}$ & $\begin{array}{l}63 . \\
65 . \\
69 . \\
60 . \\
60 .\end{array}$ & $\begin{array}{l}29 . \\
32{ }^{\circ} \\
39{ }^{\circ} \\
32{ }^{\circ} \\
39 .\end{array}$ & $\begin{array}{l}39 . \\
38 \\
46 . \\
41 . \\
48 .\end{array}$ \\
\hline $\begin{array}{l}147 \\
148 \\
149 \\
150 \\
151\end{array}$ & $\begin{array}{l}5 \\
5 \\
5 \\
5 \\
5\end{array}$ & $\begin{array}{l}27 \\
28 \\
29 \\
30 \\
31\end{array}$ & $\begin{array}{l}79 \\
79 \\
79 \\
79 \\
79\end{array}$ & $\begin{array}{l}65 . \\
66 . \\
67 . \\
66 . \\
41 .\end{array}$ & $\begin{array}{l}40 . \\
34{ }^{\circ} \\
35^{\circ} \\
32^{\circ} \\
30\end{array}$ & $\begin{array}{l}48 \\
450^{\circ} \\
48 \\
34 . \\
34 .\end{array}$ \\
\hline
\end{tabular}


May-September 1977, 1978, and 1979--Continued

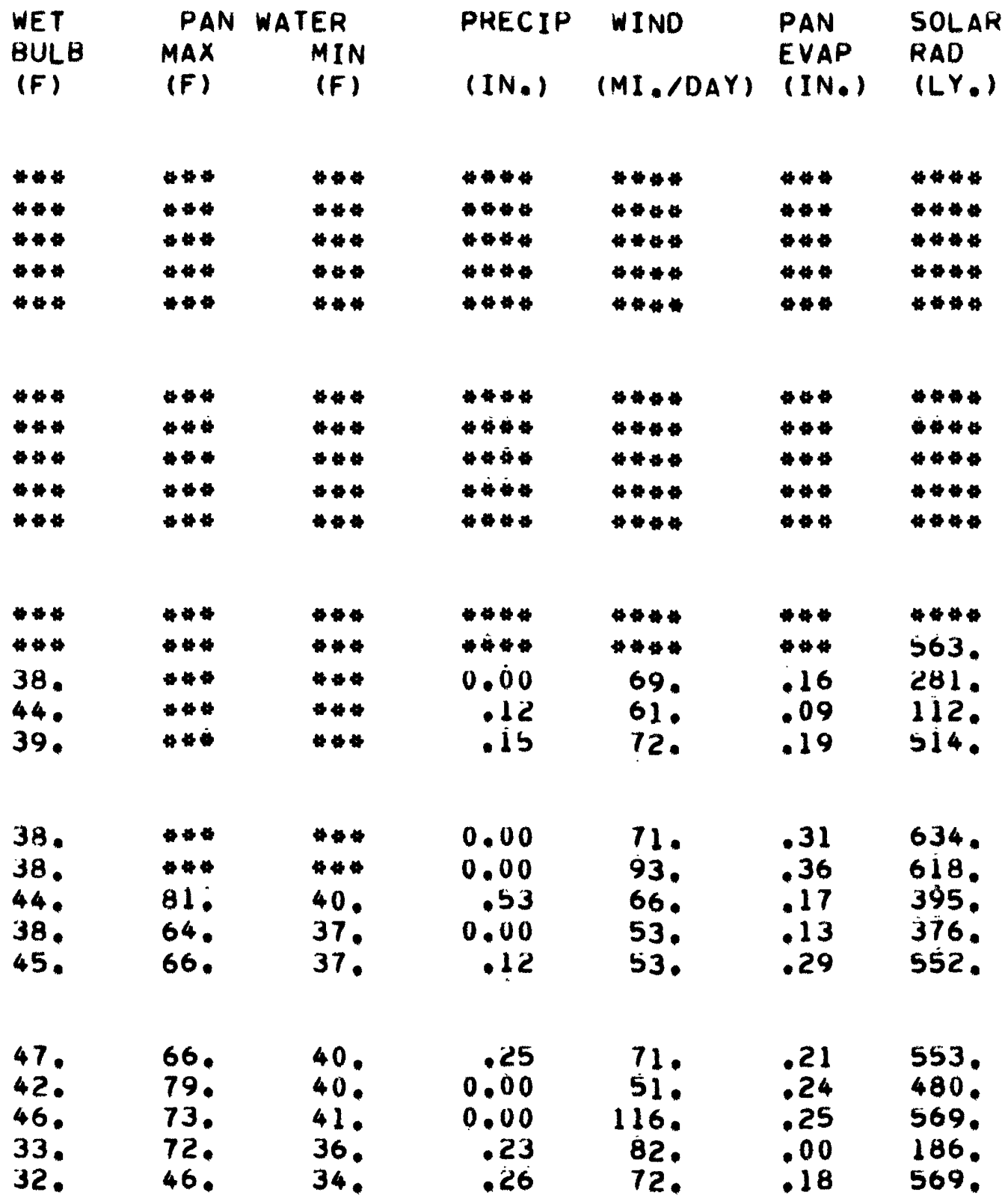


Table 6.--Climatic data, Antero Reservoir station,

\begin{tabular}{|c|c|c|c|c|c|c|}
\hline $\begin{array}{c}\text { DAY } \\
\text { OF }\end{array}$ & MONTH & DAY & YEAR & $\begin{array}{l}\text { TEMP } \\
\text { MAX }\end{array}$ & TEMPERATURE & $\begin{array}{l}\text { ORY } \\
\text { BULB }\end{array}$ \\
\hline YEAR & & & & (F) & (F) & (F) \\
\hline $\begin{array}{l}152 \\
153 \\
154 \\
155 \\
156\end{array}$ & $\begin{array}{l}6 \\
6 \\
6 \\
6 \\
6\end{array}$ & $\begin{array}{l}1 \\
2 \\
3 \\
4 \\
5\end{array}$ & $\begin{array}{l}79 \\
79 \\
79 \\
79 \\
79\end{array}$ & $\begin{array}{l}b 1 . \\
62 . \\
60 . \\
64 . \\
66 .\end{array}$ & $\begin{array}{l}32 . \\
33 . \\
33 \\
34 . \\
33 .\end{array}$ & $\begin{array}{l}36 . \\
40 . \\
35 \\
39 \\
40\end{array}$ \\
\hline $\begin{array}{l}157 \\
158 \\
159 \\
160 \\
161\end{array}$ & $\begin{array}{l}6 \\
6 \\
6 \\
6 \\
6\end{array}$ & $\begin{array}{r}6 \\
7 \\
8 \\
9 \\
10\end{array}$ & $\begin{array}{l}79 \\
79 \\
79 \\
79 \\
79\end{array}$ & $\begin{array}{l}71 . \\
71 . \\
34 . \\
38 . \\
45 .\end{array}$ & $\begin{array}{l}36 . \\
35 . \\
31 . \\
31 . \\
26 .\end{array}$ & $\begin{array}{l}41 . \\
42 \\
34 \\
32 \\
31\end{array}$ \\
\hline $\begin{array}{l}162 \\
163 \\
164 \\
165 \\
166\end{array}$ & $\begin{array}{l}6 \\
6 \\
6 \\
6 \\
6\end{array}$ & $\begin{array}{l}11 \\
12 \\
13 \\
14 \\
15\end{array}$ & $\begin{array}{l}79 \\
79 \\
79 \\
79 \\
79\end{array}$ & $\begin{array}{l}62 . \\
10 . \\
130 \\
750 \\
70 .\end{array}$ & $\begin{array}{l}30 . \\
32 . \\
35 . \\
36 . \\
36 .\end{array}$ & $\begin{array}{l}37 \\
55 \\
42 \\
43 \\
50\end{array}$ \\
\hline $\begin{array}{l}167 \\
168 \\
169 \\
170 \\
171\end{array}$ & $\begin{array}{l}6 \\
6 \\
6 \\
6 \\
6\end{array}$ & $\begin{array}{l}16 \\
17 \\
18 \\
19 \\
20\end{array}$ & $\begin{array}{l}79 \\
79 \\
79 \\
79 \\
79\end{array}$ & $\begin{array}{l}71 . \\
71 . \\
69 . \\
68 . \\
530\end{array}$ & $\begin{array}{l}40 . \\
290^{\circ} \\
31 . \\
24 . \\
36 .\end{array}$ & $\begin{array}{l}59 . \\
35 . \\
49 . \\
40 . \\
50 .\end{array}$ \\
\hline $\begin{array}{l}172 \\
173 \\
174 \\
175 \\
176\end{array}$ & $\begin{array}{l}6 \\
6 \\
6 \\
6 \\
6\end{array}$ & $\begin{array}{l}21 \\
22 \\
23 \\
24 \\
25\end{array}$ & $\begin{array}{l}79 \\
79 \\
79 \\
79 \\
19\end{array}$ & $\begin{array}{l}67 . \\
73 . \\
14 . \\
72 . \\
73 .\end{array}$ & $\begin{array}{l}29 . \\
31 . \\
33 . \\
45 . \\
32 .\end{array}$ & $\begin{array}{l}50 . \\
46 . \\
43 . \\
51 \\
36 .\end{array}$ \\
\hline
\end{tabular}


May-September 1977, 1978, and 1979--Continued

\begin{tabular}{|c|c|c|c|c|c|c|}
\hline $\begin{array}{l}\text { WET } \\
\text { BULL } \\
\text { (F) }\end{array}$ & $\begin{array}{l}\text { PAN } \\
\text { MAX } \\
\text { (F) }\end{array}$ & $\begin{array}{l}\text { WATER } \\
\text { MIN } \\
\text { (F) }\end{array}$ & $\begin{array}{l}\text { PRECIP } \\
(\text { IN.) }\end{array}$ & $\begin{array}{l}\text { WIND } \\
\text { (MI./DAY) }\end{array}$ & $\begin{array}{l}\text { PAN } \\
\text { EVAP } \\
\text { (IN.) }\end{array}$ & $\begin{array}{l}\text { SOLAR } \\
\text { RAD } \\
\text { (LY.) }\end{array}$ \\
\hline $\begin{array}{l}35 \\
39 \\
35 \\
35 \\
37\end{array}$ & $\begin{array}{l}68 . \\
66 . \\
86 . \\
77 . \\
77 .\end{array}$ & $\begin{array}{l}32 . \\
36 . \\
37 \\
41 \\
41\end{array}$ & $\begin{array}{r}0.00 \\
.26 \\
.08 \\
0.00 \\
0.00\end{array}$ & $\begin{array}{l}65 . \\
47 \\
47 \\
56 . \\
45\end{array}$ & $\begin{array}{l}.15 \\
.22 \\
.35 \\
.20 \\
.29\end{array}$ & $\begin{array}{l}459 . \\
625 . \\
590 . \\
518 . \\
612 .\end{array}$ \\
\hline $\begin{array}{l}39 . \\
42 . \\
32 . \\
32 \\
30\end{array}$ & $\begin{array}{l}77 . \\
79 . \\
75 . \\
43 . \\
75 .\end{array}$ & $\begin{array}{l}41 . \\
41 . \\
36 \\
32 \\
32 \\
\end{array}$ & $\begin{array}{r}0.00 \\
0.00 \\
.67 \\
.57 \\
.26\end{array}$ & $\begin{array}{r}99 . \\
118 . \\
55 . \\
76 . \\
37 .\end{array}$ & $\begin{array}{l}.41 \\
\$ 4 * \\
.44 \\
.40 \\
.24\end{array}$ & $\begin{array}{l}642 . \\
282 . \\
160^{\circ} \\
553^{\circ} \\
703^{\circ}\end{array}$ \\
\hline $\begin{array}{l}34 \\
51 . \\
38 \\
42 \\
44 .\end{array}$ & $\begin{array}{l}79 . \\
860^{\circ} \\
86 . \\
79 . \\
75 .\end{array}$ & $\begin{array}{l}34 \\
39 \\
45 \\
43 \\
41\end{array}$ & $\begin{array}{l}0.00 \\
0.00 \\
0.00 \\
0.00 \\
0.00\end{array}$ & $\begin{array}{l}55 . \\
16 . \\
57 . \\
90 \\
82 .\end{array}$ & $\begin{array}{l}.32 \\
.19 \\
.35 \\
.26 \\
.21\end{array}$ & $\begin{array}{l}624 \\
602 . \\
580 \\
471 \\
475\end{array}$ \\
\hline $\begin{array}{l}43 . \\
30 \\
40 \\
39 \\
40\end{array}$ & $\begin{array}{l}81 . \\
77 . \\
73 . \\
68 . \\
63 .\end{array}$ & $\begin{array}{l}39 . \\
370^{\circ} \\
370^{\circ} \\
36 . \\
36 .\end{array}$ & $\begin{array}{l}0.00 \\
0.00 \\
.03 \\
0.00 \\
0.00\end{array}$ & $\begin{array}{l}90 . \\
1110^{\circ} \\
207 . \\
166 . \\
71 .\end{array}$ & $\begin{array}{l}.41 \\
.50 \\
.41 \\
.29 \\
.30\end{array}$ & $\begin{array}{l}652 . \\
729 . \\
632 . \\
588 . \\
670 .\end{array}$ \\
\hline $\begin{array}{l}46 . \\
39 \\
39 \\
49 \\
40\end{array}$ & $\begin{array}{l}75 . \\
81 . \\
79 . \\
75 . \\
73 .\end{array}$ & $\begin{array}{l}37 . \\
39 . \\
39 . \\
37 . \\
41 .\end{array}$ & $\begin{array}{l}0.00 \\
0.00 \\
0.00 \\
0.00 \\
0.00\end{array}$ & $\begin{array}{l}55 . \\
64 . \\
81 . \\
53 . \\
57 .\end{array}$ & $\begin{array}{l}.36 \\
.19 \\
.10 \\
.28 \\
.27\end{array}$ & $\begin{array}{l}706 . \\
495 . \\
441 . \\
393 . \\
514 .\end{array}$ \\
\hline
\end{tabular}


Table 6.--Climatic data, Antero Reservoir station,

\begin{tabular}{|c|c|c|c|c|c|c|}
\hline $\begin{array}{r}\text { DAY } \\
\text { OF } \\
\text { YEAR }\end{array}$ & MONTH & DAY & YEAR & $\begin{array}{l}\text { TEMI } \\
\text { MAX } \\
\text { (F) }\end{array}$ & $\begin{array}{l}\text { TURE } \\
\text { MIN } \\
\text { (F) }\end{array}$ & $\begin{array}{l}\text { DRY } \\
\text { BULB } \\
\text { (F) }\end{array}$ \\
\hline $\begin{array}{l}177 \\
178 \\
179 \\
180 \\
181\end{array}$ & $\begin{array}{l}6 \\
6 \\
6 \\
6 \\
6\end{array}$ & $\begin{array}{l}26 \\
27 \\
28 \\
29 \\
30\end{array}$ & $\begin{array}{l}79 \\
79 \\
79 \\
79 \\
79\end{array}$ & $\begin{array}{l}75 . \\
77 . \\
16 . \\
17 . \\
80 .\end{array}$ & $\begin{array}{l}31 . \\
36 . \\
37 . \\
37 . \\
40 .\end{array}$ & $\begin{array}{l}48 . \\
49 . \\
49 \\
47 \\
57\end{array}$ \\
\hline $\begin{array}{l}182 \\
183 \\
184 \\
185 \\
186\end{array}$ & $\begin{array}{l}7 \\
7 \\
7 \\
7 \\
7\end{array}$ & $\begin{array}{l}1 \\
2 \\
3 \\
4 \\
5\end{array}$ & $\begin{array}{l}79 \\
79 \\
79 \\
79 \\
79\end{array}$ & $\begin{array}{l}75 . \\
72 . \\
72 . \\
73 . \\
72 .\end{array}$ & $\begin{array}{l}48 . \\
35 . \\
38 . \\
35 . \\
39 .\end{array}$ & $\begin{array}{l}520^{\circ} \\
41^{\circ} \\
43{ }^{\circ} \\
45{ }^{\circ}\end{array}$ \\
\hline $\begin{array}{l}187 \\
188 \\
189 \\
190 \\
191\end{array}$ & $\begin{array}{l}7 \\
7 \\
7 \\
7 \\
7\end{array}$ & $\begin{array}{r}6 \\
7 \\
8 \\
9 \\
10\end{array}$ & $\begin{array}{l}79 \\
79 \\
79 \\
79 \\
79\end{array}$ & $\begin{array}{l}72 . \\
72 . \\
77 . \\
75 . \\
77 .\end{array}$ & $\begin{array}{l}37 . \\
33 . \\
37 \\
35 \\
30\end{array}$ & $\begin{array}{l}50 \\
42 . \\
44{ }^{\circ} \\
42 \\
42 .\end{array}$ \\
\hline $\begin{array}{l}192 \\
193 \\
194 \\
195 \\
196\end{array}$ & $\begin{array}{l}7 \\
7 \\
7 \\
7 \\
7\end{array}$ & $\begin{array}{l}11 \\
12 \\
13 \\
14 \\
15\end{array}$ & $\begin{array}{l}79 \\
79 \\
79 \\
79 \\
79\end{array}$ & $\begin{array}{l}80 . \\
82 . \\
81 . \\
81 . \\
82 .\end{array}$ & $\begin{array}{l}35 . \\
38 \\
35 \\
40 \\
43\end{array}$ & $\begin{array}{l}43 . \\
47 . \\
48 . \\
55 \\
52 .\end{array}$ \\
\hline $\begin{array}{l}197 \\
198 \\
199 \\
200 \\
201\end{array}$ & $\begin{array}{l}7 \\
7 \\
7 \\
7 \\
7\end{array}$ & $\begin{array}{l}16 \\
17 \\
18 \\
19 \\
20\end{array}$ & $\begin{array}{l}79 \\
79 \\
79 \\
79 \\
79\end{array}$ & $\begin{array}{l}72 . \\
74 . \\
15 . \\
68 . \\
72 .\end{array}$ & $\begin{array}{l}42 . \\
38 . \\
35 . \\
33 . \\
33 .\end{array}$ & $\begin{array}{l}46 . \\
45 \circ^{\circ} \\
470^{\circ} \\
38 \\
46 .\end{array}$ \\
\hline
\end{tabular}


May-September 1977, 1978, and 1979--Continued

$\begin{array}{lllllll}\text { WET } & \text { PAN WATER } & \text { PRECIP } & \text { WIND } & \text { PAN } & \text { SOLAR } \\ \text { BULB } & \text { MAX } & \text { MIN } & & & \text { EVAP } & \text { RAD } \\ \text { (F) } & \text { (F) } & \text { (F) } & \text { (IN.) } & \text { (MI.IDAY) } & \text { (IN.) } & \text { (LY.) }\end{array}$

\begin{tabular}{|c|c|c|c|c|c|}
\hline $\begin{array}{l}43 . \\
48^{\circ} \\
450^{\circ} \\
49 .\end{array}$ & $\begin{array}{l}77 . \\
75 . \\
770 \\
770 \\
81 .\end{array}$ & $\begin{array}{l}41 . \\
43 . \\
43 . \\
43 . \\
43 .\end{array}$ & $\begin{array}{l}0.00 \\
0.00 \\
0.00 \\
0.00 \\
0.00\end{array}$ & $\begin{array}{l}52 \\
51 \\
42 \\
47 \\
82\end{array}$ & $\begin{array}{l}.25 \\
.38 \\
.24 \\
.29 \\
.30\end{array}$ \\
\hline
\end{tabular}

$\begin{array}{lllllll}48 . & 77 . & 46 . & 0.00 & 48 . & .12 & 264 . \\ 41 . & 70 . & 41 . & .12 & 56 . & .29 & 517 . \\ 45 . & 75 . & 41 . & 0.00 & 52 . & .21 & 396 . \\ 42 . & 77 . & 43 . & 0.00 & 49 . & .41 & 539 . \\ 45 . & 84 . & 43 . & 0.00 & 50 . & .15 & 494 .\end{array}$

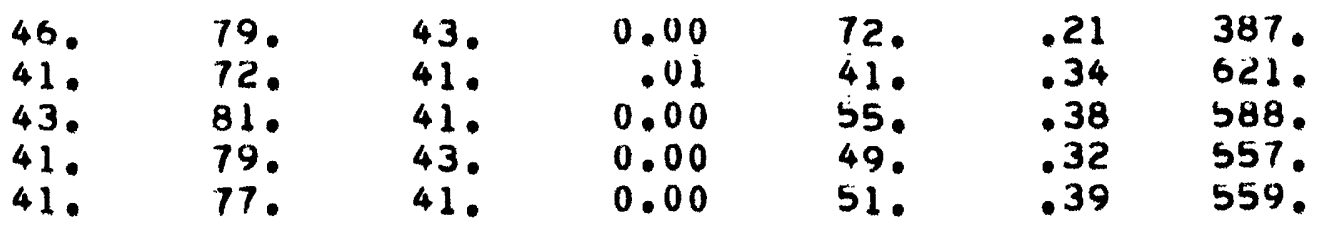

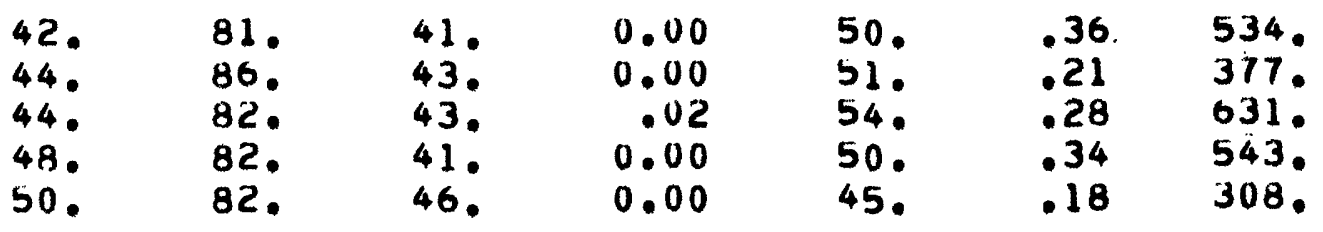

$\begin{array}{lllllll}45 . & 72 . & 46 . & .24 & 56 . & .23 & 354 . \\ 44 . & 72 . & 45 . & .15 & 55 . & .23 & 433 . \\ 44 . & 73 . & 43 . & .05 & 48 . & .15 & 317 . \\ 35 . & 66 . & 39 . & .15 & 47 . & .24 & 559 . \\ 44 . & 79 . & 39 . & .04 & 41 . & .20 & 480 .\end{array}$


Table 6.--Climatic data, Antero Reservoir station,

\begin{tabular}{|c|c|c|c|c|c|c|}
\hline $\begin{array}{l}\text { DAY } \\
\text { OF } \\
\text { YEAR }\end{array}$ & MONTH & DAY & YEAR & $\begin{array}{l}\text { TEMP } \\
\text { MAX } \\
\text { (F) }\end{array}$ & $\begin{array}{l}\text { IURE } \\
\text { MIN } \\
\text { (F) }\end{array}$ & $\begin{array}{l}\text { DRY } \\
\text { BULB } \\
\text { (F) }\end{array}$ \\
\hline $\begin{array}{l}202 \\
203 \\
204 \\
205 \\
206\end{array}$ & $\begin{array}{l}7 \\
7 \\
7 \\
7 \\
7\end{array}$ & $\begin{array}{l}21 \\
22 \\
23 \\
24 \\
25\end{array}$ & $\begin{array}{l}79 \\
79 \\
79 \\
79 \\
79\end{array}$ & $\begin{array}{l}74 . \\
75 \\
74 \\
77 \\
75\end{array}$ & $\begin{array}{l}37 \circ^{\circ} \\
48 \circ^{\circ} \\
34 \circ^{\circ} \\
35 \circ^{\circ} \\
43 .\end{array}$ & $\begin{array}{l}47 . \\
56 . \\
42 . \\
48 \\
54 .\end{array}$ \\
\hline $\begin{array}{l}207 \\
208 \\
209 \\
210 \\
211\end{array}$ & $\begin{array}{l}7 \\
7 \\
7 \\
7 \\
7\end{array}$ & $\begin{array}{l}26 \\
27 \\
28 \\
29 \\
30\end{array}$ & $\begin{array}{l}79 \\
79 \\
79 \\
79 \\
79\end{array}$ & $\begin{array}{l}77 . \\
78 . \\
78 . \\
81 \\
75 .\end{array}$ & $\begin{array}{l}360^{\circ} \\
360^{\circ} \\
370^{\circ} \\
350^{\circ}\end{array}$ & $\begin{array}{l}51 . \\
50 . \\
47 . \\
55 . \\
44 .\end{array}$ \\
\hline $\begin{array}{l}212 \\
213 \\
214 \\
215 \\
216\end{array}$ & $\begin{array}{l}7 \\
8 \\
8 \\
8 \\
8\end{array}$ & $\begin{array}{r}31 \\
1 \\
2 \\
3 \\
4\end{array}$ & $\begin{array}{l}79 \\
79 \\
79 \\
79 \\
79\end{array}$ & $\begin{array}{l}77 . \\
750 \\
78 . \\
79 . \\
80 .\end{array}$ & $\begin{array}{l}36 . \\
31 . \\
33 \circ \\
39 \\
32 .\end{array}$ & $\begin{array}{l}46 . \\
41 \\
47 \\
46 . \\
49\end{array}$ \\
\hline $\begin{array}{l}217 \\
218 \\
219 \\
220 \\
221\end{array}$ & $\begin{array}{l}8 \\
8 \\
8 \\
8 \\
8\end{array}$ & $\begin{array}{l}5 \\
6 \\
7 \\
8 \\
9\end{array}$ & $\begin{array}{l}79 \\
79 \\
79 \\
79 \\
79\end{array}$ & $\begin{array}{l}83 . \\
830^{\circ} \\
850^{\circ} \\
84 \circ^{\circ} \\
80 .\end{array}$ & $\begin{array}{l}34{ }^{\circ} \\
330^{\circ} \\
360^{\circ} \\
40^{\circ} \\
44 \circ^{\circ}\end{array}$ & $\begin{array}{l}49 . \\
43{ }^{\circ} \\
470^{\circ} \\
51 . \\
55 .\end{array}$ \\
\hline $\begin{array}{l}222 \\
223 \\
224 \\
225 \\
226\end{array}$ & $\begin{array}{l}8 \\
8 \\
8 \\
8 \\
8\end{array}$ & $\begin{array}{l}10 \\
11 \\
12 \\
13 \\
14\end{array}$ & $\begin{array}{l}79 \\
79 \\
79 \\
79 \\
79\end{array}$ & $\begin{array}{l}13 . \\
65 . \\
75 . \\
77 \\
13 .\end{array}$ & $\begin{array}{l}440^{\circ} \\
420^{\circ} \\
390^{\circ} \\
430^{\circ}\end{array}$ & $\begin{array}{l}51 \\
48 \\
49 \\
45 \\
50\end{array}$ \\
\hline
\end{tabular}


May-September 1977, 1978, and 1979--Continued

\begin{tabular}{|c|c|c|c|c|c|}
\hline $\begin{array}{l}\text { WET } \\
\text { BULB } \\
(F)\end{array}$ & $\begin{array}{l}\text { PAN } \\
\text { MAX } \\
\text { (F) }\end{array}$ & $\begin{array}{l}\text { WATER } \\
\text { MIN } \\
\text { (F) }\end{array}$ & $\begin{array}{l}\text { PRECIP } \\
\left(I N_{\bullet}\right)\end{array}$ & $\begin{array}{l}\text { WIND } \\
\text { (MI./DAY) }\end{array}$ & $\begin{array}{l}\text { PAN } \\
\text { EVAP } \\
\text { (IN.) }\end{array}$ \\
\hline
\end{tabular}

$\begin{array}{lllllll}45 . & 79 . & 43 . & 0.00 & 51 . & .32 & 464 . \\ 51 . & 79 . & 48 . & 0.00 & 60 . & .32 & 589 . \\ 42 . & 79 . & 52 . & 0.00 & 33 . & .68 & 472 . \\ 46 . & 79 . & 41 . & 0.00 & 43 . & .18 & 403 . \\ 49 . & 77 . & 46 . & 0.00 & 51 . & .18 & 431 .\end{array}$

$\begin{array}{lllllll}47 . & 77 . & 43 . & 0.00 & 52 . & .18 & 379 . \\ 47 . & 79 . & 41 . & 0.00 & 55 . & .23 & 386 . \\ 47 . & 70 . & 43 . & .07 & 58 . & .24 & 454 . \\ 50 . & 77 . & 45 . & 0.00 & 51 . & .35 & 435 . \\ 42 . & 73 . & 43 . & .08 & 60 . & .28 & 438 .\end{array}$

\begin{tabular}{|c|c|c|c|c|c|}
\hline $\begin{array}{l}45 . \\
41 . \\
41 . \\
42 . \\
41\end{array}$ & $\begin{array}{l}73 . \\
75 . \\
79 . \\
82 . \\
79 .\end{array}$ & $\begin{array}{l}43 . \\
41 . \\
41 . \\
41 .\end{array}$ & $\begin{array}{r}0.00 \\
.01 \\
0.00 \\
0.00 \\
0.00\end{array}$ & $\begin{array}{l}55 . \\
57 . \\
44 . \\
44 . \\
46 .\end{array}$ & $\begin{array}{l}.26 \\
.35 \\
.34 \\
.27 \\
.46\end{array}$ \\
\hline
\end{tabular}

$\begin{array}{lllllll}45 . & 81 . & 45 . & 0.00 & 44 . & .42 & 557 . \\ 43 . & 81 . & 43 . & 0.00 & 44 . & .27 & 444 . \\ 42 . & 79 . & 43 . & 0.00 & 37 . & .31 & 533 . \\ 44 . & 86 . & 43 . & 0.00 & 52 . & .32 & 384 . \\ 48 . & 79 . & 46 . & 0.00 & 53 . & .12 & 308 . \\ & & & & & & \\ 49 . & 70 . & 46 . & .09 & 54 . & .12 & 520 . \\ 47 . & 68 . & 46 . & .15 & 46 . & .31 & 428 . \\ 48 . & 82 . & 46 . & 0.00 & 53 . & .19 & 347 . \\ 45 . & 75 . & 46 . & 0.00 & 71 . & .23 & 348 . \\ 50 . & 70 . & 46 . & .92 & 45 . & .08 & 132 .\end{array}$


Table 6.--Climatic data, Antero Reservoir station,

\begin{tabular}{|c|c|c|c|c|c|c|}
\hline $\begin{array}{c}\text { DAY } \\
\text { OF } \\
\text { YEAR }\end{array}$ & MONTH & DAY & YEAR & $\begin{array}{l}\text { TEMP } \\
\text { MAX } \\
\text { (F) }\end{array}$ & $\begin{array}{l}\text { TREE } \\
\text { MIN } \\
\text { (F) }\end{array}$ & $\begin{array}{l}\text { DRY } \\
\text { BULB } \\
\text { (F) }\end{array}$ \\
\hline $\begin{array}{l}227 \\
228 \\
229 \\
230 \\
231\end{array}$ & $\begin{array}{l}8 \\
8 \\
8 \\
8 \\
8\end{array}$ & $\begin{array}{l}15 \\
16 \\
17 \\
18 \\
19\end{array}$ & $\begin{array}{l}79 \\
79 \\
79 \\
79 \\
79\end{array}$ & $\begin{array}{l}55 . \\
62 . \\
68 . \\
67 . \\
64 .\end{array}$ & $\begin{array}{l}46 . \\
460^{\circ} \\
38 \\
38 \\
34\end{array}$ & $\begin{array}{l}49 . \\
490^{\circ} \\
420^{\circ} \\
47{ }^{\circ}\end{array}$ \\
\hline $\begin{array}{l}232 \\
233 \\
234 \\
235 \\
236\end{array}$ & $\begin{array}{l}8 \\
8 \\
8 \\
8 \\
8\end{array}$ & $\begin{array}{l}20 \\
21 \\
22 \\
23 \\
24\end{array}$ & $\begin{array}{l}79 \\
79 \\
79 \\
79 \\
79\end{array}$ & $\begin{array}{l}62 . \\
60 . \\
66 . \\
69 . \\
70 .\end{array}$ & $\begin{array}{l}32 . \\
31 . \\
31 . \\
34 \circ \\
36 .\end{array}$ & $\begin{array}{l}38 \\
39 \\
42 \\
42 \\
43\end{array}$ \\
\hline $\begin{array}{l}237 \\
238 \\
239 \\
240 \\
241\end{array}$ & $\begin{array}{l}8 \\
8 \\
8 \\
8 \\
8\end{array}$ & $\begin{array}{l}25 \\
26 \\
27 \\
28 \\
29\end{array}$ & $\begin{array}{l}79 \\
79 \\
79 \\
79 \\
79\end{array}$ & $\begin{array}{l}10 . \\
68 \\
67 \\
12 \\
73\end{array}$ & $\begin{array}{l}38 . \\
37 . \\
32 . \\
31 . \\
29 .\end{array}$ & $\begin{array}{l}450^{\circ} \\
450^{\circ} \\
36 . \\
430^{\circ}\end{array}$ \\
\hline $\begin{array}{l}242 \\
243 \\
244 \\
245 \\
246\end{array}$ & $\begin{array}{l}8 \\
8 \\
9 \\
9 \\
9\end{array}$ & $\begin{array}{r}30 \\
31 \\
1 \\
2 \\
3\end{array}$ & $\begin{array}{l}79 \\
79 \\
79 \\
79 \\
79\end{array}$ & $\begin{array}{l}76 . \\
71 . \\
70 \\
75 . \\
74 .\end{array}$ & $\begin{array}{l}34{ }^{\circ} \\
34 \circ^{\circ} \\
260^{\circ} \\
300^{\circ} \\
290^{\circ}\end{array}$ & $\begin{array}{l}42 . \\
44 \\
38 \\
38 \\
38 \\
.\end{array}$ \\
\hline $\begin{array}{l}247 \\
248 \\
249 \\
250 \\
251\end{array}$ & $\begin{array}{l}9 \\
9 \\
9 \\
9 \\
9\end{array}$ & $\begin{array}{l}4 \\
5 \\
6 \\
7 \\
8\end{array}$ & $\begin{array}{l}79 \\
79 \\
79 \\
79 \\
79\end{array}$ & $\begin{array}{l}16 . \\
770^{\circ} \\
78 . \\
78 . \\
18 .\end{array}$ & $\begin{array}{l}34 . \\
28 . \\
32 . \\
41 . \\
33 .\end{array}$ & $\begin{array}{l}44 . \\
370^{\circ} \\
42 . \\
450^{\circ} \\
42 .\end{array}$ \\
\hline
\end{tabular}


May-September 1977, 1978, and 1979--Continued

$\begin{array}{lllllll}\text { WET } & \text { PAN WATER } & \text { PRECIP } & \text { WIND } & \text { PAN } & \text { SOLAR } \\ \text { BULB } & \text { MAX } & \text { MIN } & & & \text { EVAP } & \text { RAD } \\ \text { (F) } & \text { (F) } & \text { (F) } & \text { (IN.) } & \text { (MI./DAY) } & \text { (IN.) } & \text { (LY.) }\end{array}$

$\begin{array}{lllllll}47 . & 72 . & 46 . & .73 & 86 . & .21 & 367 . \\ 47 . & 66 . & 46 . & .03 & 69 . & .17 & 477 . \\ 41 . & 75 . & 45 . & 0.00 & 48 . & .22 & 377 . \\ 46 . & 72 . & 43 . & .03 & 46 . & .09 & 294 . \\ 43 . & 68 . & 39 . & .05 & 75 . & .31 & 586 .\end{array}$

$\begin{array}{lllllll}38 . & 70 . & 39 . & 0.00 & 73 . & .18 & 481 . \\ 40 . & 66 . & 37 . & 0.00 & 52 . & .19 & 387 . \\ 42 . & 64 . & 37 . & 0.00 & 34 . & .23 & 382 . \\ 42 . & 75 . & 37 . & 0.00 & 36 . & .14 & 423 . \\ 41 . & 77 . & 41 . & 0.00 & 42 . & .20 & 459 .\end{array}$

$\begin{array}{lllllll}43 . & 75 . & 41 . & 0.00 & 50 . & .25 & 380 . \\ 43 . & 68 . & 41 . & 0.00 & 48 . & .14 & 340 . \\ 36 . & 66 . & 37 . & 0.00 & 44 . & .14 & 490 . \\ 40 . & 73 . & 37 . & 0.00 & 59 . & .38 & 527 . \\ 36 . & 73 . & 37 . & 0.00 & 39 . & .20 & 498 .\end{array}$

$\begin{array}{lllllll}40 . & 79 . & 37 . & 0.00 & 57 . & .20 & 373 . \\ 42 . & 66 . & 37 . & .08 & 60 . & .24 & 589 . \\ 35 . & 73 . & 36 . & 0.00 & 51 . & .35 & 562 . \\ 38 . & 73 . & 36 . & 0.00 & 47 . & .22 & 478 . \\ 36 . & 73 . & 39 . & 0.00 & 27 . & .31 & 431 .\end{array}$

$\begin{array}{lllllll}43 . & 75 . & 37 . & 0.00 & 41 . & .19 & 510 . \\ 34 . & 79 . & 39 . & 0.00 & 27 . & .29 & 441 . \\ 38 . & 79 . & 37 . & 0.00 & 33 . & .23 & 366 . \\ 44 . & 75 . & 41 . & 0.00 & 27 . & .14 & 399 . \\ 39 . & 81 . & 41 . & 0.00 & 24 . & .28 & 325 .\end{array}$


Table 6.--Climatic data, Antero Reservoir station,

\begin{tabular}{|c|c|c|c|c|c|c|}
\hline $\begin{array}{r}\text { DAY } \\
\text { OF } \\
\text { YEAR }\end{array}$ & MONTH & DAY & YEAR & $\begin{array}{l}\text { TEMP } \\
\text { MAX } \\
\text { (F) }\end{array}$ & $\begin{array}{l}\text { URE } \\
\text { MIN } \\
\text { (F) }\end{array}$ & $\begin{array}{l}\text { DRY } \\
\text { BULB } \\
\text { (F) }\end{array}$ \\
\hline $\begin{array}{l}252 \\
253 \\
254 \\
255 \\
256\end{array}$ & $\begin{array}{l}9 \\
9 \\
9 \\
9 \\
9\end{array}$ & $\begin{array}{l}9 \\
10 \\
11 \\
12 \\
13\end{array}$ & $\begin{array}{l}19 \\
79 \\
79 \\
79 \\
19\end{array}$ & $\begin{array}{l}78 . \\
77 \\
76 \\
71 \\
65\end{array}$ & $\begin{array}{l}32{ }^{\circ} \\
34{ }^{\circ} \\
36{ }^{\circ} \\
30 \\
32 .\end{array}$ & $\begin{array}{l}27 . \\
420^{\circ} \\
420^{\circ} \\
34{ }^{\circ} \\
42 .\end{array}$ \\
\hline $\begin{array}{l}257 \\
258 \\
259 \\
260 \\
261\end{array}$ & $\begin{array}{l}9 \\
9 \\
9 \\
9 \\
9\end{array}$ & $\begin{array}{l}14 \\
15 \\
16 \\
17 \\
18\end{array}$ & $\begin{array}{l}79 \\
19 \\
79 \\
79 \\
19\end{array}$ & $\begin{array}{l}62 . \\
51 . \\
67 . \\
72 . \\
72 .\end{array}$ & $\begin{array}{l}320^{\circ} \\
140^{\circ} \\
190^{\circ} \\
210^{\circ} \\
23 .\end{array}$ & $\begin{array}{l}35 . \\
32 \\
35 \\
27 \\
31\end{array}$ \\
\hline $\begin{array}{l}262 \\
263 \\
264 \\
265 \\
266\end{array}$ & $\begin{array}{l}9 \\
9 \\
9 \\
9 \\
9\end{array}$ & $\begin{array}{l}19 \\
20 \\
21 \\
22 \\
23\end{array}$ & $\begin{array}{l}79 \\
79 \\
79 \\
79 \\
79\end{array}$ & $\begin{array}{l}65 . \\
71 . \\
60 . \\
64 . \\
65 .\end{array}$ & $\begin{array}{l}220^{\circ} \\
260^{\circ} \\
36{ }^{\circ} \\
29{ }^{\circ} \\
27 .\end{array}$ & $\begin{array}{l}28 . \\
* 4 \\
* * 4 \\
* 4\end{array}$ \\
\hline $\begin{array}{l}267 \\
268 \\
269 \\
270 \\
271\end{array}$ & $\begin{array}{l}9 \\
9 \\
9 \\
9 \\
9\end{array}$ & $\begin{array}{l}24 \\
25 \\
26 \\
27 \\
28\end{array}$ & $\begin{array}{l}79 \\
79 \\
79 \\
79 \\
79\end{array}$ & $\begin{array}{l}71 . \\
72 . \\
67 . \\
64 . \\
62 .\end{array}$ & $\begin{array}{l}26 . \\
34{ }^{\circ} \\
29{ }^{\circ} \\
28 . \\
21 .\end{array}$ & $\begin{array}{l}1 \% \\
42 . \\
34 . \\
34 . \\
28 .\end{array}$ \\
\hline $\begin{array}{l}272 \\
273\end{array}$ & $\begin{array}{l}9 \\
9\end{array}$ & $\begin{array}{l}29 \\
30\end{array}$ & $\begin{array}{l}79 \\
79\end{array}$ & $\begin{array}{l}68 . \\
71 .\end{array}$ & $\begin{array}{l}24^{\circ} \\
24{ }^{\circ}\end{array}$ & $\begin{array}{l}29 . \\
30 .\end{array}$ \\
\hline
\end{tabular}


May-September 1977, 1978, and 1979--Continued

\begin{tabular}{|c|c|c|c|c|c|}
\hline $\begin{array}{l}\text { WET } \\
\text { BULB } \\
\text { (F) }\end{array}$ & $\begin{array}{l}\text { PAN } \\
\text { MAX } \\
\text { (F) }\end{array}$ & $\begin{array}{r}\text { WATER } \\
\text { MI } \\
\text { IF }\end{array}$ & $\begin{array}{l}\text { PRECIP } \\
\left(I N_{0}\right)\end{array}$ & $\begin{array}{l}\text { WIND } \\
\text { (MI./DAY) }\end{array}$ & $\begin{array}{l}\text { PAN } \\
\text { EVAP } \\
\text { (IN.) }\end{array}$ \\
\hline
\end{tabular}

$\begin{array}{lllllll}36 . & 73 . & 41 . & 0.00 & 25 . & .16 & 291 . \\ 41 . & 70 . & 39 . & 0.00 & 46 . & .21 & 351 . \\ 41 . & 73 . & 41 . & .10 & 63 . & .25 & 373 . \\ 34 . & 70 . & 39 . & 0.00 & 83 . & .22 & 462 . \\ 39 . & 70 . & 37 . & 0.00 & 74 . & .09 & 268 .\end{array}$

\begin{tabular}{|c|c|c|c|c|c|c|}
\hline $\begin{array}{l}34 . \\
22 \\
32 . \\
26 . \\
29 .\end{array}$ & $\begin{array}{l}63 . \\
72 . \\
68 . \\
72 . \\
68 .\end{array}$ & $\begin{array}{l}36 . \\
32 \\
30 \\
32 \\
30\end{array}$ & $\begin{array}{l}.07 \\
0.00 \\
0.00 \\
0.00 \\
0.00\end{array}$ & $\begin{array}{l}31 . \\
23 . \\
34 . \\
61 . \\
39 .\end{array}$ & $\begin{array}{l}* * 4 \\
* * * \\
.24 \\
.22 \\
.24\end{array}$ & $\begin{array}{l}248 . \\
497 \\
477 \\
418 \\
397\end{array}$ \\
\hline $\begin{array}{l}26 . \\
* * 4 \\
* * 4 \\
* * 4\end{array}$ & $\begin{array}{l}68 . \\
66 . \\
61 . \\
72 . \\
63 .\end{array}$ & $\begin{array}{l}34 . \\
32 \\
28 \\
28 \\
36\end{array}$ & $\begin{array}{r}0.00 \\
0.00 \\
.037 \\
0.00 \\
0.00\end{array}$ & $\begin{array}{r}48 . \\
48, \\
* * * * \\
* * * *\end{array}$ & $\begin{array}{l}.14 \\
.14 \\
.15 \\
.16 \\
.20\end{array}$ & $\begin{array}{l}363 . \\
242 . \\
425, \\
307 . \\
366 .\end{array}$ \\
\hline $\begin{array}{l}4 \% \\
40 . \\
33 . \\
33 . \\
28 .\end{array}$ & $\begin{array}{l}72 . \\
70 . \\
66 . \\
68 . \\
59 .\end{array}$ & $\begin{array}{l}36 . \\
360^{\circ} \\
370^{\circ} \\
36 . \\
34 .\end{array}$ & $\begin{array}{l}0.00 \\
0.00 \\
0.00 \\
0.00 \\
0.00\end{array}$ & 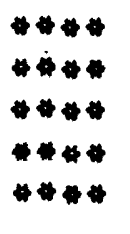 & $\begin{array}{l}.12 \\
.13 \\
.27 \\
.06 \\
.11\end{array}$ & $\begin{array}{l}373 . \\
302 . \\
350 . \\
317 \\
382 .\end{array}$ \\
\hline $\begin{array}{l}9.9 \\
00\end{array}$ & $\begin{array}{l}68 . \\
70 .\end{array}$ & $\begin{array}{l}34 . \\
34 .\end{array}$ & $\begin{array}{l}0.00 \\
0.00\end{array}$ & \#*** & $\begin{array}{r}.31 \\
.17\end{array}$ & $\begin{array}{l}377 . \\
346 .\end{array}$ \\
\hline
\end{tabular}



Table 7.--Climatic data, Garo station, May-September 1977 and 1978

\author{
PRECIP: Daily precipitation \\ DAILY AVG TEMP: Daily average air temperature \\ DAILY AVG REL HUM: Daily average relative humidity \\ SOLAR RAD: Daily average solar radiation \\ F: Degrees Fahrenheit \\ IN: Inches \\ MI: Mile \\ LY: Langleys per minute \\ $\star \star \star$ : Represents missing data
}


Table 7.--Climatic data, Garo station, May-September 1977 and 1978--Continued

\begin{tabular}{|c|c|c|c|c|c|c|c|c|}
\hline $\begin{array}{l}\text { DAY } \\
\text { OF } \\
\text { YEAR }\end{array}$ & MONTH & DAY & YEAR & $\begin{array}{l}\text { PRECIP } \\
\left(I N_{0}\right)\end{array}$ & $\begin{array}{c}\text { WIND } \\
(M I . / O A Y)\end{array}$ & $\begin{array}{l}\text { DAIL } \\
\text { TEMP } \\
\text { (F) }\end{array}$ & $\begin{array}{l}\text { AVG } \\
\text { REL HUM } \\
\text { (PERCENT) }\end{array}$ & $\begin{array}{l}\text { SOLAR } \\
\text { RAD } \\
\text { (LY.) }\end{array}$ \\
\hline $\begin{array}{l}121 \\
122 \\
123 \\
124 \\
125\end{array}$ & $\begin{array}{l}5 \\
5 \\
5 \\
5 \\
5\end{array}$ & $\begin{array}{l}1 \\
2 \\
3 \\
4 \\
5\end{array}$ & $\begin{array}{l}77 \\
77 \\
77 \\
77 \\
77\end{array}$ & $\begin{array}{l}0.00 \\
0.00 \\
0.00 \\
0.00 \\
0.00\end{array}$ & $\begin{array}{l}* * * * \\
* * * * \\
* * * * \\
* * * * \\
* * * *\end{array}$ & $\begin{array}{l}* * * * \\
* * * * \\
* * * \\
* * * \\
* * *\end{array}$ & 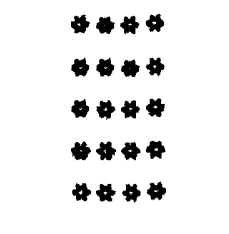 & 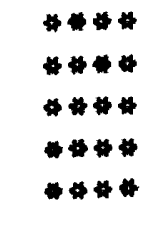 \\
\hline $\begin{array}{l}126 \\
127 \\
128 \\
129 \\
130\end{array}$ & $\begin{array}{l}5 \\
5 \\
5 \\
5 \\
5\end{array}$ & $\begin{array}{r}6 \\
7 \\
8 \\
9 \\
10\end{array}$ & $\begin{array}{l}77 \\
77 \\
77 \\
77 \\
77\end{array}$ & $\begin{array}{l}0.00 \\
0.00 \\
0.00 \\
0.00 \\
0.00\end{array}$ & 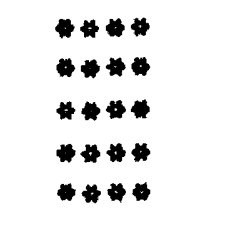 & $\begin{array}{l}\# * * * \\
* * * * \\
* * * * \\
* * * * \\
* * * *\end{array}$ & 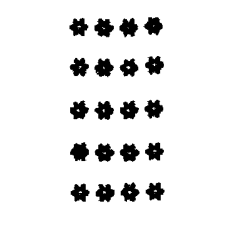 & 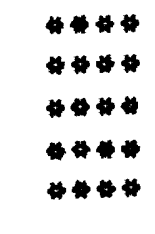 \\
\hline $\begin{array}{l}131 \\
132 \\
133 \\
134 \\
135\end{array}$ & $\begin{array}{l}5 \\
5 \\
5 \\
5 \\
5\end{array}$ & $\begin{array}{l}11 \\
12 \\
13 \\
14 \\
15\end{array}$ & $\begin{array}{l}77 \\
77 \\
77 \\
77 \\
77\end{array}$ & $\begin{array}{l}0.00 \\
0.00 \\
0.00 \\
0.00 \\
0.00\end{array}$ & 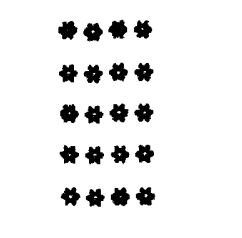 & $\begin{array}{l}* * * * \\
* * * 4 \\
* * * 4 \\
* * * *\end{array}$ & $\begin{array}{l}* * 4 * \\
* * 4 * \\
* * 4 \\
* * 4 \\
* * 4\end{array}$ & 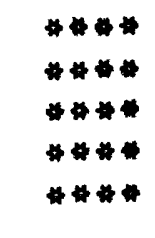 \\
\hline $\begin{array}{l}136 \\
137 \\
138 \\
139 \\
140\end{array}$ & $\begin{array}{l}5 \\
5 \\
5 \\
5 \\
5\end{array}$ & $\begin{array}{l}16 \\
17 \\
18 \\
19 \\
20\end{array}$ & $\begin{array}{l}77 \\
77 \\
77 \\
77 \\
77\end{array}$ & $\begin{array}{l}0.00 \\
0.00 \\
0.00 \\
0.00 \\
0.00\end{array}$ & 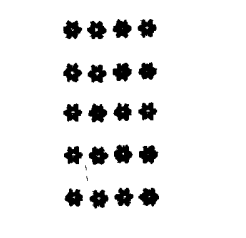 & 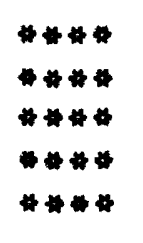 & 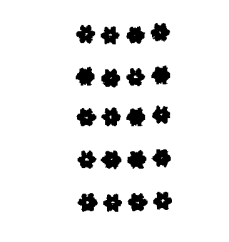 & $\begin{array}{l}* * 4 * \\
* * * * \\
* * * 4 \\
* * * * \\
* * * *\end{array}$ \\
\hline $\begin{array}{l}141 \\
142 \\
143 \\
144 \\
145\end{array}$ & $\begin{array}{l}5 \\
5 \\
5 \\
5 \\
5\end{array}$ & $\begin{array}{l}21 \\
22 \\
23 \\
24 \\
25\end{array}$ & $\begin{array}{l}77 \\
77 \\
77 \\
77 \\
77\end{array}$ & $\begin{array}{l}0.00 \\
0.00 \\
0.00 \\
0.00 \\
0.00\end{array}$ & 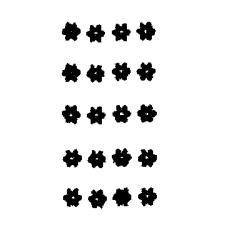 & 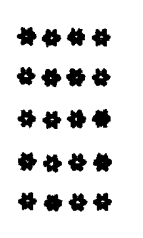 & 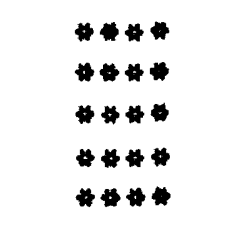 & 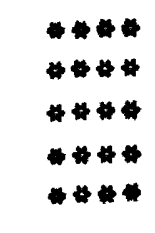 \\
\hline
\end{tabular}


Table 7.--Climatic data, Garo station, May-September 1977 and 1978--Continued

\begin{tabular}{|c|c|c|c|c|c|c|c|c|}
\hline $\begin{array}{r}\text { DAY } \\
\text { OF } \\
\text { YEAR }\end{array}$ & MONTH & DAY & YEAR & $\begin{array}{l}\text { PRECIP } \\
\text { (IN.) }\end{array}$ & $\begin{array}{l}\text { WIND } \\
\text { (MI./DAY) }\end{array}$ & $\begin{array}{l}\text { DAIL } \\
\text { TEMP } \\
\text { (F) }\end{array}$ & $\begin{array}{l}\text { AVG } \\
\text { REL HUM } \\
\text { (PERCENT) }\end{array}$ & $\begin{array}{l}\text { SOLAR } \\
\text { RAD } \\
(L Y .)\end{array}$ \\
\hline $\begin{array}{l}146 \\
147 \\
148 \\
149 \\
150\end{array}$ & $\begin{array}{l}5 \\
5 \\
5 \\
5 \\
5\end{array}$ & $\begin{array}{l}26 \\
27 \\
28 \\
29 \\
30\end{array}$ & $\begin{array}{l}77 \\
77 \\
77 \\
77 \\
77\end{array}$ & $\begin{array}{l}0.00 \\
0.00 \\
0.00 \\
0.00 \\
0.00\end{array}$ & 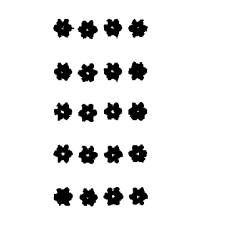 & $\begin{array}{l}* * * * \\
* * * 4 \\
* * * * \\
* * * 4 \\
* * * *\end{array}$ & 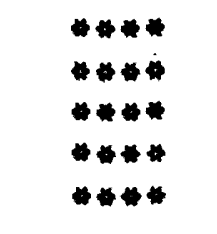 & 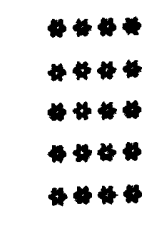 \\
\hline $\begin{array}{l}151 \\
152 \\
153 \\
154 \\
155\end{array}$ & $\begin{array}{l}5 \\
6 \\
6 \\
6 \\
6\end{array}$ & $\begin{array}{r}31 \\
1 \\
2 \\
3 \\
4\end{array}$ & $\begin{array}{l}77 \\
77 \\
77 \\
77 \\
77\end{array}$ & $\begin{array}{l}0.00 \\
0.00 \\
0.00 \\
0.00 \\
0.00\end{array}$ & 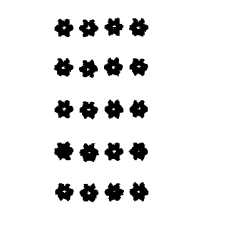 & 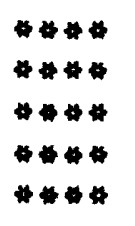 & 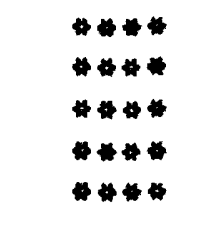 & 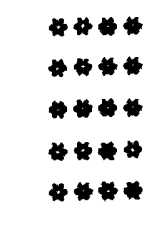 \\
\hline $\begin{array}{l}156 \\
157 \\
158 \\
159 \\
160\end{array}$ & $\begin{array}{l}6 \\
6 \\
6 \\
6 \\
6\end{array}$ & $\begin{array}{l}5 \\
6 \\
7 \\
8 \\
9\end{array}$ & $\begin{array}{l}77 \\
77 \\
77 \\
77 \\
77\end{array}$ & $\begin{array}{r}0.00 \\
0.00 \\
0.00 \\
0.00 \\
.11\end{array}$ & 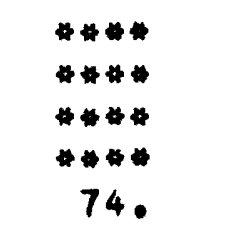 & 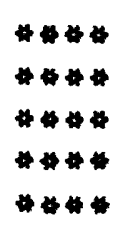 & 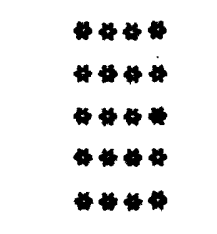 & 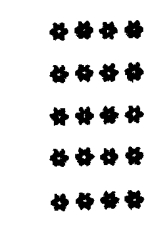 \\
\hline $\begin{array}{l}161 \\
162 \\
163 \\
164 \\
165\end{array}$ & $\begin{array}{l}6 \\
6 \\
6 \\
6 \\
6\end{array}$ & $\begin{array}{l}10 \\
11 \\
12 \\
13 \\
14\end{array}$ & $\begin{array}{l}77 \\
77 \\
77 \\
77 \\
77\end{array}$ & $\begin{array}{r}0.00 \\
.04 \\
.04 \\
0.00 \\
.31\end{array}$ & $\begin{array}{l}71 . \\
66 . \\
68 . \\
68 . \\
55 .\end{array}$ & 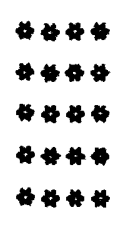 & 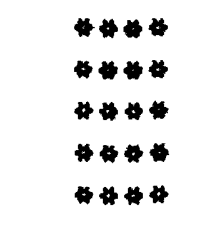 & 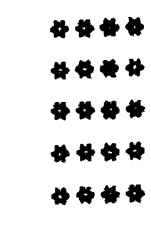 \\
\hline $\begin{array}{l}166 \\
167 \\
168 \\
169 \\
170\end{array}$ & $\begin{array}{l}6 \\
6 \\
6 \\
6 \\
6\end{array}$ & $\begin{array}{l}15 \\
16 \\
17 \\
18 \\
19\end{array}$ & $\begin{array}{l}77 \\
77 \\
77 \\
77 \\
77\end{array}$ & $\begin{array}{l}0.00 \\
0.00 \\
0.00 \\
0.00 \\
0.00\end{array}$ & $\begin{array}{r}79 \\
64 \\
155^{\circ} \\
112^{\circ}\end{array}$ & 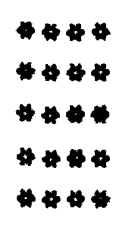 & 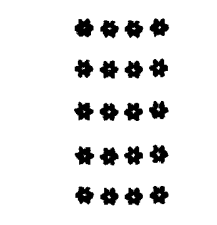 & 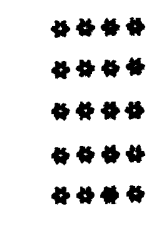 \\
\hline
\end{tabular}


Table 7.--Climatic data, Garo station, May-September 1977 and 1978--Continued

\begin{tabular}{|c|c|c|c|c|c|c|c|c|}
\hline $\begin{array}{r}\text { DAY } \\
\text { OF } \\
\text { YEAR }\end{array}$ & MONTH & DAY & YEAR & $\begin{array}{l}\text { PRECIP } \\
\text { (IN.) }\end{array}$ & $\begin{array}{l}\text { WIND } \\
\text { (MI./DAY) }\end{array}$ & $\begin{array}{l}\text { DAIL } \\
\text { TEMP } \\
\text { (F) }\end{array}$ & $\begin{array}{l}Y \text { AVG } \\
\text { REL HUM } \\
\text { (PERCENT) }\end{array}$ & $\begin{array}{l}\text { SOLAR } \\
\text { RAD } \\
\left(L Y_{\bullet}\right)\end{array}$ \\
\hline $\begin{array}{l}171 \\
172 \\
173 \\
174 \\
175\end{array}$ & $\begin{array}{l}6 \\
6 \\
6 \\
6 \\
6\end{array}$ & $\begin{array}{l}20 \\
21 \\
22 \\
23 \\
24\end{array}$ & $\begin{array}{l}77 \\
77 \\
77 \\
77 \\
77\end{array}$ & $\begin{array}{r}0.00 \\
0.00 \\
0.00 \\
0.00 \\
.01\end{array}$ & $\begin{array}{l}64 . \\
650^{\circ} \\
540^{\circ} \\
580^{\circ} \\
62 .\end{array}$ & 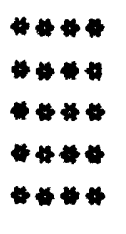 & 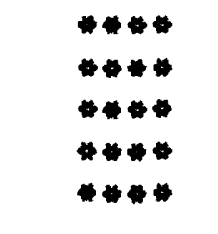 & 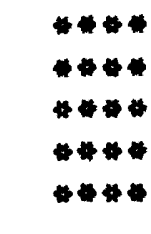 \\
\hline $\begin{array}{l}176 \\
177 \\
178 \\
179 \\
180\end{array}$ & $\begin{array}{l}6 \\
6 \\
6 \\
6 \\
6\end{array}$ & $\begin{array}{l}25 \\
26 \\
27 \\
28 \\
29\end{array}$ & $\begin{array}{l}77 \\
77 \\
77 \\
77 \\
77\end{array}$ & $\begin{array}{r}.01 \\
.02 \\
.02 \\
0.00 \\
0.00\end{array}$ & $\begin{array}{r}67 \\
74 \\
136 \\
54 \\
6\end{array}$ & 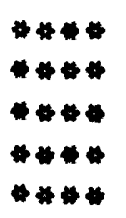 & $\begin{array}{l}* * * * \\
* * * * \\
* * * * \\
* * * * \\
* * * *\end{array}$ & 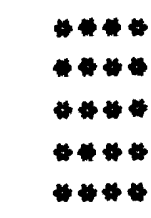 \\
\hline $\begin{array}{l}181 \\
182 \\
183 \\
184 \\
185\end{array}$ & $\begin{array}{l}6 \\
7 \\
7 \\
7 \\
7\end{array}$ & $\begin{array}{r}30 \\
1 \\
2 \\
3 \\
4\end{array}$ & $\begin{array}{l}77 \\
77 \\
77 \\
77 \\
77\end{array}$ & $\begin{array}{l}0.00 \\
0.00 \\
0.00 \\
0.00 \\
0.00\end{array}$ & $\begin{array}{r}144 . \\
87 . \\
78 \\
90 \\
81\end{array}$ & $\begin{array}{l}* * * * \\
* * * * \\
* * * * \\
* * * 4 \\
* * * *\end{array}$ & 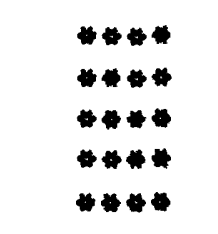 & 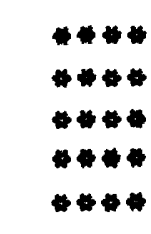 \\
\hline $\begin{array}{l}186 \\
187 \\
188 \\
189 \\
190\end{array}$ & $\begin{array}{l}7 \\
7 \\
7 \\
7 \\
7\end{array}$ & $\begin{array}{l}5 \\
6 \\
7 \\
8 \\
9\end{array}$ & $\begin{array}{l}77 \\
77 \\
77 \\
77 \\
17\end{array}$ & $\begin{array}{r}.01 \\
.05 \\
0.00 \\
0.00 \\
0.00\end{array}$ & $\begin{array}{l}26 . \\
48 . \\
580^{\circ} \\
76 . \\
73 .\end{array}$ & 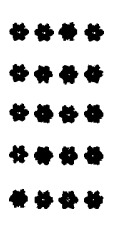 & 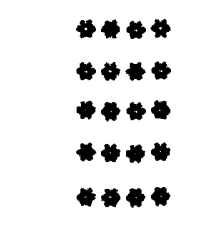 & 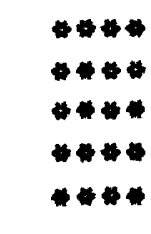 \\
\hline $\begin{array}{l}191 \\
192 \\
193 \\
194 \\
195\end{array}$ & $\begin{array}{l}7 \\
7 \\
7 \\
7 \\
7\end{array}$ & $\begin{array}{l}10 \\
11 \\
12 \\
13 \\
14\end{array}$ & $\begin{array}{l}77 \\
77 \\
77 \\
77 \\
77\end{array}$ & $\begin{array}{r}.03 \\
0.00 \\
0.00 \\
0.00 \\
.01\end{array}$ & $\begin{array}{r}52 \\
56 \\
135 \\
54 \\
34\end{array}$ & 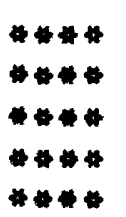 & 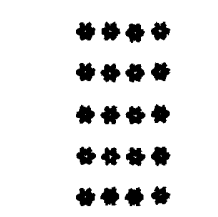 & $\begin{array}{l}* * * * \\
* * * * \\
* * * * \\
* * * *\end{array}$ \\
\hline
\end{tabular}


Table 7.--Climatic data, Garo station, May-September 1977 and 1978--Continued

\begin{tabular}{|c|c|c|c|c|c|c|c|c|}
\hline $\begin{array}{r}\text { DAY } \\
\text { OF } \\
\text { YEAR }\end{array}$ & MONTH & DAY & YEAR & $\begin{array}{l}\text { PRECIP } \\
(\text { IN.) }\end{array}$ & $\begin{array}{c}\text { WIND } \\
\text { (MI./OAY) }\end{array}$ & $\begin{array}{l}\text { DAILY } \\
\text { TEMP } \\
\text { (F) }\end{array}$ & $\begin{array}{l}\text { Y AVG } \\
\text { REL HUM } \\
\text { (PERCENT) }\end{array}$ & $\begin{array}{l}\text { SOLAF } \\
\text { RAD } \\
\text { (LY,) }\end{array}$ \\
\hline
\end{tabular}

\begin{tabular}{|c|c|c|c|c|c|c|c|}
\hline $\begin{array}{l}196 \\
197 \\
198 \\
199 \\
200\end{array}$ & $\begin{array}{l}7 \\
7 \\
7 \\
7 \\
7\end{array}$ & $\begin{array}{l}15 \\
16 \\
17 \\
18 \\
19\end{array}$ & $\begin{array}{l}77 \\
77 \\
77 \\
77 \\
77\end{array}$ & $\begin{array}{l}.01 \\
0.00 \\
0.00 \\
0.00 \\
0.00\end{array}$ & $\begin{array}{l}36 . \\
86 . \\
55 . \\
35 . \\
61 .\end{array}$ & 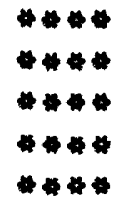 & 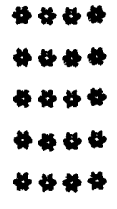 \\
\hline
\end{tabular}

201

202

203

204

205

206

207

208

209

210

211

212

213

214

215

216

217

218

219

220

\section{0}

21

22

23

24

25

26

27

28

29

30

31

1

?

3

$\begin{array}{ll}4 & 77 \\ 5 & 77 \\ 6 & 77 \\ 7 & 77 \\ 8 & 77\end{array}$

\section{7}

77

77

77

77

77

77

77

77

77

77

77

77

77

77

77

77

77

77

$\begin{array}{rl}.60 & 24 . \\ .33 & 49 \\ .06 & 69 \\ 0.00 & 62 \\ .15 & 26 .\end{array}$

\section{.33}

.62

.44

0.00

0.00

0.00

0.00

0.00

0.00

0.00

0.00

.19

0.00

0.00

0.00

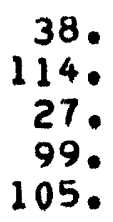

26.

36.

67.

76.

46.

56.

71.

61.

76.

61.
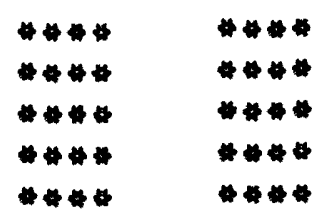

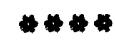

$\forall$ 수 숭

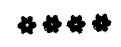

$4 * 4$

$4 * 4$

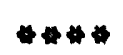

-4*4

57.

58.

61.

*4*

70.

63.

60.

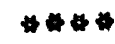

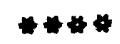

521.

490.

491.

63.

59.

64.

59.

60.

46.

43.

65.

39.

\#*4

57.

59.

62.

60.

59.

68.

76.

54.

50 .

61.
719.

752. 647. 648 . 465.

318. 518. 579. 455 . 353. 
Table 7.--Climatic data, Garo station, May-September 1977 and 1978--Continued

\begin{tabular}{|c|c|c|c|c|c|c|c|c|}
\hline $\begin{array}{l}\text { DAY } \\
\text { OF } \\
\text { YEAR }\end{array}$ & MONTH & DAY & YEAR & $\begin{array}{l}\text { PRECIP } \\
\text { (IN.) }\end{array}$ & $\begin{array}{c}\text { WIND } \\
\text { (MI./DAY) }\end{array}$ & $\begin{array}{l}\text { DAII } \\
\text { TEMP } \\
\text { (F) }\end{array}$ & $\begin{array}{l}\text { Y AVG } \\
\text { REL HUM } \\
\text { (PERCENT) }\end{array}$ & $\begin{array}{l}\text { SOLAR } \\
\text { RAD } \\
\text { (LY.) }\end{array}$ \\
\hline $\begin{array}{l}221 \\
222 \\
223 \\
224 \\
225\end{array}$ & $\begin{array}{l}8 \\
8 \\
8 \\
8 \\
8\end{array}$ & $\begin{array}{r}9 \\
10 \\
11 \\
12 \\
13\end{array}$ & $\begin{array}{l}77 \\
77 \\
77 \\
77 \\
77\end{array}$ & $\begin{array}{r}0.00 \\
.12 \\
.29 \\
0.00 \\
0.00\end{array}$ & $\begin{array}{l}41 . \\
12 . \\
72 . \\
51 . \\
64 .\end{array}$ & $\begin{array}{l}62 . \\
59 . \\
56 . \\
58 . \\
58 .\end{array}$ & $\begin{array}{l}76 . \\
790^{\circ} \\
730^{\circ} \\
54 . \\
58 .\end{array}$ & $\begin{array}{l}553 . \\
426 . \\
4740^{\circ} \\
492 . \\
484 .\end{array}$ \\
\hline $\begin{array}{l}226 \\
227 \\
228 \\
229 \\
230\end{array}$ & $\begin{array}{l}8 \\
8 \\
8 \\
8 \\
8\end{array}$ & $\begin{array}{l}14 \\
15 \\
16 \\
17 \\
18\end{array}$ & $\begin{array}{l}77 \\
77 \\
77 \\
77 \\
77\end{array}$ & $\begin{array}{l}0.00 \\
.03 \\
.57 \\
.35 \\
.01\end{array}$ & $\begin{array}{l}44 . \\
42 . \\
30 \\
60 \\
68 .\end{array}$ & $\begin{array}{l}57 . \\
57 . \\
58 . \\
57 . \\
57 .\end{array}$ & $\begin{array}{l}63 . \\
740^{\circ} \\
90 . \\
86 . \\
75 .\end{array}$ & $\begin{array}{l}510 . \\
292 . \\
262 . \\
248 . \\
359 .\end{array}$ \\
\hline $\begin{array}{l}231 \\
232 \\
233 \\
234 \\
235\end{array}$ & $\begin{array}{l}8 \\
8 \\
8 \\
8 \\
8\end{array}$ & $\begin{array}{l}19 \\
20 \\
21 \\
22 \\
23\end{array}$ & $\begin{array}{l}77 \\
77 \\
77 \\
77 \\
77\end{array}$ & $\begin{array}{r}.02 \\
.01 \\
0.00 \\
.10 \\
0.00\end{array}$ & $\begin{array}{l}60 . \\
46 . \\
51 . \\
80 . \\
56 .\end{array}$ & $\begin{array}{l}56 . \\
59 . \\
55 . \\
57 . \\
57 .\end{array}$ & $\begin{array}{l}84 . \\
720^{\circ} \\
770^{\circ} \\
68 \\
62 .\end{array}$ & $\begin{array}{l}373 . \\
396 . \\
405 . \\
* * * \\
* * *\end{array}$ \\
\hline $\begin{array}{l}236 \\
237 \\
238 \\
239 \\
240\end{array}$ & $\begin{array}{l}8 \\
8 \\
8 \\
8 \\
8\end{array}$ & $\begin{array}{l}24 \\
25 \\
26 \\
27 \\
28\end{array}$ & $\begin{array}{l}77 \\
77 \\
77 \\
77 \\
77\end{array}$ & $\begin{array}{l}0.00 \\
0.00 \\
0.00 \\
.01 \\
0.00\end{array}$ & $\begin{array}{l}60 . \\
630^{\circ} \\
950^{\circ} \\
750^{\circ} \\
41 .\end{array}$ & $\begin{array}{l}60 . \\
58 . \\
53 . \\
56 . \\
60 .\end{array}$ & $\begin{array}{l}57 . \\
58 . \\
650^{\circ} \\
59 \\
64 .\end{array}$ & $\begin{array}{l}* \# * \\
4 * * \\
458 . \\
448 \text {. } \\
402 \text {. }\end{array}$ \\
\hline $\begin{array}{l}241 \\
242 \\
243 \\
244 \\
245\end{array}$ & $\begin{array}{l}8 \\
8 \\
8 \\
9 \\
9\end{array}$ & $\begin{array}{r}29 \\
30 \\
31 \\
1 \\
2\end{array}$ & $\begin{array}{l}77 \\
77 \\
77 \\
77 \\
77\end{array}$ & $\begin{array}{l}0.00 \\
0.00 \\
0.00 \\
0.00 \\
0.00\end{array}$ & $\begin{array}{l}80 . \\
60 . \\
66 . \\
88 . \\
49 .\end{array}$ & $\begin{array}{l}58 . \\
60 . \\
63 . \\
54 . \\
55 .\end{array}$ & $\begin{array}{l}75{ }^{\circ} \\
64 . \\
520^{\circ} \\
52 . \\
74 .\end{array}$ & $\begin{array}{l}415 . \\
579 \\
540 \\
465 \\
\end{array}$ \\
\hline
\end{tabular}


Table 7.--Climatic data, Garo station, May-September 1977 and 1978--Continued

\begin{tabular}{|c|c|c|c|c|c|c|c|c|}
\hline $\begin{array}{l}\text { DAY } \\
\text { OF } \\
\text { YEAR }\end{array}$ & MONTH & DAY & YEAR & $\begin{array}{l}\text { PRECIP } \\
\text { (IN.) }\end{array}$ & $\begin{array}{l}\text { WINO } \\
\text { (MI./DAY) }\end{array}$ & $\begin{array}{l}\text { DAIL } \\
\text { TEMP } \\
\text { (F) }\end{array}$ & $\begin{array}{l}\text { Y AVG } \\
\text { REL HUM } \\
\text { (PERCENT) }\end{array}$ & $\begin{array}{l}\text { SOLAR } \\
\text { RAD } \\
\left(L Y_{\bullet}\right)\end{array}$ \\
\hline $\begin{array}{l}246 \\
247 \\
248 \\
249 \\
250\end{array}$ & $\begin{array}{l}9 \\
9 \\
9 \\
9 \\
9\end{array}$ & $\begin{array}{l}3 \\
4 \\
5 \\
6 \\
7\end{array}$ & $\begin{array}{l}77 \\
77 \\
77 \\
77 \\
77\end{array}$ & $\begin{array}{r}.01 \\
0.00 \\
.12 \\
0.00 \\
.10\end{array}$ & $\begin{array}{l}53 . \\
55 \\
32 \\
40 \\
41 .\end{array}$ & $\begin{array}{l}54 . \\
55{ }^{\circ} \\
53 . \\
55 . \\
57 .\end{array}$ & $\begin{array}{l}82 . \\
48 . \\
52 . \\
65 . \\
52 .\end{array}$ & $\begin{array}{l}* \# * 4 \\
* * 4 \\
34 * 4 \\
360 . \\
448 .\end{array}$ \\
\hline $\begin{array}{l}251 \\
252 \\
253 \\
254 \\
255\end{array}$ & $\begin{array}{l}9 \\
9 \\
9 \\
9 \\
9\end{array}$ & $\begin{array}{r}8 \\
9 \\
10 \\
11 \\
12\end{array}$ & $\begin{array}{l}77 \\
77 \\
77 \\
77 \\
77\end{array}$ & $\begin{array}{r}0.00 \\
0.00 \\
.01 \\
.01 \\
.07\end{array}$ & $\begin{array}{l}99 . \\
84 \circ^{\circ} \\
38 . \\
110^{\circ} \\
41 .\end{array}$ & $\begin{array}{c}4 * \\
51 . \\
51 . \\
49 .\end{array}$ & $\begin{array}{r}* \# \# \\
\# * \# \\
69 . \\
93 . \\
68 .\end{array}$ & $\begin{array}{l}564^{\circ} \\
369^{\circ} \\
367^{\circ} \\
327^{\circ} \\
450^{\circ}\end{array}$ \\
\hline $\begin{array}{l}256 \\
257 \\
258 \\
259 \\
260\end{array}$ & $\begin{array}{l}9 \\
9 \\
9 \\
9 \\
9\end{array}$ & $\begin{array}{l}13 \\
14 \\
15 \\
16 \\
17\end{array}$ & $\begin{array}{l}77 \\
77 \\
77 \\
77 \\
77\end{array}$ & $\begin{array}{l}0.00 \\
0.00 \\
0.00 \\
0.00 \\
0.00\end{array}$ & $\begin{array}{r}83 . \\
116 . \\
53 \\
189 \\
31 .\end{array}$ & $\begin{array}{l}46 . \\
48 \\
50 \\
48 \\
52\end{array}$ & $\begin{array}{l}74 \\
65 \\
66 \\
60 \\
47\end{array}$ & $\begin{array}{l}451 . \\
393^{\circ} \\
468^{\circ} \\
442^{\circ} \\
475 .\end{array}$ \\
\hline $\begin{array}{l}261 \\
262 \\
263 \\
264 \\
265\end{array}$ & $\begin{array}{l}9 \\
9 \\
9 \\
9 \\
9\end{array}$ & $\begin{array}{l}18 \\
19 \\
20 \\
21 \\
22\end{array}$ & $\begin{array}{l}77 \\
77 \\
77 \\
77 \\
77\end{array}$ & $\begin{array}{l}0.00 \\
0.00 \\
0.00 \\
0.00 \\
0.00\end{array}$ & $\begin{array}{r}25 . \\
10 \\
85 \\
176 \\
116 .\end{array}$ & $\begin{array}{l}50 \\
50 \\
* * * \\
* * * \\
* * *\end{array}$ & $\begin{array}{r}45 . \\
54 . \\
* 4 * \\
* * 4\end{array}$ & $\begin{array}{l}491^{\circ} \\
428^{\circ} \\
524^{\circ} \\
4620^{\circ} \\
470^{\circ}\end{array}$ \\
\hline $\begin{array}{l}266 \\
267 \\
268 \\
269 \\
270\end{array}$ & $\begin{array}{l}9 \\
9 \\
9 \\
9 \\
9\end{array}$ & $\begin{array}{l}23 \\
24 \\
25 \\
26 \\
27\end{array}$ & $\begin{array}{l}77 \\
77 \\
77 \\
77 \\
77\end{array}$ & $\begin{array}{l}0.00 \\
0.00 \\
0.00 \\
0.00 \\
0.00\end{array}$ & 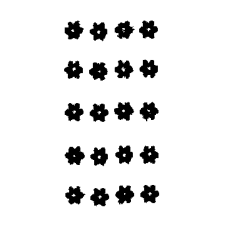 & $\begin{array}{l}\$ 4 \$ \\
50 \\
56 \\
53 \\
50\end{array}$ & $\begin{array}{l}\$ 4 \\
26 . \\
52 . \\
52 \\
54\end{array}$ & $\begin{array}{l}504 \\
451 . \\
498 \\
340 \\
408\end{array}$ \\
\hline
\end{tabular}


Table 7.--Climatic data, Garo station, May-September 1977 and 1978--Continued

\begin{tabular}{|c|c|c|c|c|c|c|c|c|}
\hline $\begin{array}{r}\text { DAY } \\
\text { OF } \\
\text { YEAR }\end{array}$ & MONTH & DAY & YEAR & $\begin{array}{l}\text { PRECIP } \\
\text { (IN.) }\end{array}$ & $\begin{array}{l}\text { WIND } \\
\text { (MI./DAY) }\end{array}$ & $\begin{array}{l}\text { DAIL } \\
\text { TEMP } \\
\text { (F) }\end{array}$ & $\begin{array}{l}\text { Y AVG } \\
\text { REL HUM } \\
\text { (PERCENT) }\end{array}$ & $\begin{array}{l}\text { SOLAR } \\
\text { RAD } \\
(L Y,)\end{array}$ \\
\hline $\begin{array}{l}271 \\
272 \\
273 \\
274 \\
121\end{array}$ & $\begin{array}{r}9 \\
9 \\
9 \\
10 \\
5\end{array}$ & $\begin{array}{r}28 \\
29 \\
30 \\
1 \\
1\end{array}$ & $\begin{array}{l}77 \\
77 \\
77 \\
77 \\
78\end{array}$ & $\begin{array}{l}0.00 \\
0.00 \\
0.00 \\
0.00 \\
.03\end{array}$ & $\begin{array}{r}* 4 * \\
* * 4 \\
* 4 * \\
* 4 * \\
65 .\end{array}$ & $\begin{array}{r}50 \\
50 \\
45 \\
40 \\
31\end{array}$ & $\begin{array}{l}52 \\
47 \\
55 \\
86\end{array}$ & $\begin{array}{l}297^{\circ} \\
374^{\circ} \\
356 . \\
268 .\end{array}$ \\
\hline $\begin{array}{l}122 \\
123 \\
124 \\
125 \\
126\end{array}$ & $\begin{array}{l}5 \\
5 \\
5 \\
5 \\
5\end{array}$ & $\begin{array}{l}2 \\
3 \\
4 \\
5 \\
6\end{array}$ & $\begin{array}{l}78 \\
78 \\
78 \\
78 \\
78\end{array}$ & $\begin{array}{r}.26 \\
.01 \\
0.00 \\
.03 \\
.18\end{array}$ & $\begin{array}{r}57 \\
140 \\
114 \\
169^{\circ} \\
95^{\circ}\end{array}$ & $\begin{array}{l}30 . \\
36 \\
28 \\
28 \\
29\end{array}$ & $\begin{array}{l}81 . \\
64 . \\
73 . \\
76 \\
85\end{array}$ & $\begin{array}{l}359 . \\
533 \\
475 \\
420 \\
295 .\end{array}$ \\
\hline $\begin{array}{l}127 \\
128 \\
129 \\
130 \\
131\end{array}$ & $\begin{array}{l}5 \\
5 \\
5 \\
5 \\
5\end{array}$ & $\begin{array}{r}7 \\
8 \\
9 \\
10 \\
11\end{array}$ & $\begin{array}{l}78 \\
78 \\
78 \\
78 \\
78\end{array}$ & $\begin{array}{r}0.00 \\
.01 \\
0.00 \\
0.00 \\
.99\end{array}$ & $\begin{array}{r}138 \\
86 \\
34 \\
99 \\
99\end{array}$ & $\begin{array}{l}20 \\
27 \\
36 \\
44 \\
43\end{array}$ & $\begin{array}{l}68 . \\
580^{\circ} \\
360^{\circ} \\
38 . \\
53 .\end{array}$ & $\begin{array}{l}678 . \\
461 . \\
696 . \\
654 . \\
585 .\end{array}$ \\
\hline $\begin{array}{l}132 \\
133 \\
134 \\
135 \\
136\end{array}$ & $\begin{array}{l}5 \\
5 \\
5 \\
5 \\
5\end{array}$ & $\begin{array}{l}12 \\
13 \\
14 \\
15 \\
16\end{array}$ & $\begin{array}{l}78 \\
78 \\
78 \\
78 \\
78\end{array}$ & $\begin{array}{l}.05 \\
0.00 \\
0.00 \\
0.00 \\
0.00\end{array}$ & $\begin{array}{r}69 \\
114 \\
77 \\
75 \\
100\end{array}$ & $\begin{array}{l}38 \\
46 \\
49 \\
53 \\
52\end{array}$ & $\begin{array}{l}41 . \\
32 . \\
42 . \\
27 . \\
36 .\end{array}$ & $\begin{array}{l}746 . \\
7040^{\circ} \\
696 . \\
660 . \\
732 .\end{array}$ \\
\hline $\begin{array}{l}137 \\
138 \\
139 \\
140 \\
141\end{array}$ & $\begin{array}{l}5 \\
5 \\
5 \\
5 \\
5\end{array}$ & $\begin{array}{l}17 \\
18 \\
19 \\
20 \\
21\end{array}$ & $\begin{array}{l}78 \\
78 \\
78 \\
78 \\
78\end{array}$ & $\begin{array}{l}0.00 \\
0.00 \\
0.00 \\
0.00 \\
.01\end{array}$ & $\begin{array}{r}186 . \\
66 \\
60 \\
71 \\
94\end{array}$ & $\begin{array}{l}41 \\
39 \\
46 \\
43 \\
41\end{array}$ & $\begin{array}{l}51 . \\
50 . \\
330^{\circ} \\
80 \\
74 .\end{array}$ & $\begin{array}{l}400 \\
750 \\
606 . \\
341 . \\
419 .\end{array}$ \\
\hline
\end{tabular}


Table 7.--Climatic data, Garo station, May-September 1977 and 1978--Continued

\begin{tabular}{|c|c|c|c|c|c|c|c|c|}
\hline $\begin{array}{r}\text { DAY } \\
\text { OF } \\
\text { YEAR }\end{array}$ & MONTH & DAY & YEAR & $\begin{array}{l}\text { PRECIP } \\
(\text { IN.) }\end{array}$ & $\begin{array}{c}\text { WIND } \\
\text { (MI./OAY) }\end{array}$ & $\begin{array}{l}\text { DAIL } \\
\text { TEMP } \\
\text { (F) }\end{array}$ & $\begin{array}{l}\text { Y AVG } \\
\text { REL HUM } \\
\text { (PERCENT) }\end{array}$ & $\begin{array}{l}\text { SOLAR } \\
\text { RAD } \\
(L Y,)\end{array}$ \\
\hline $\begin{array}{l}142 \\
143 \\
144 \\
145 \\
146\end{array}$ & $\begin{array}{l}5 \\
5 \\
5 \\
5 \\
5\end{array}$ & $\begin{array}{l}22 \\
23 \\
24 \\
25 \\
26\end{array}$ & $\begin{array}{l}78 \\
78 \\
78 \\
78 \\
78\end{array}$ & $\begin{array}{l}0.00 \\
0.00 \\
0.00 \\
0.00 \\
0.00\end{array}$ & $\begin{array}{r}103 . \\
82 . \\
110 . \\
85 . \\
129 .\end{array}$ & $\begin{array}{l}46 . \\
46 \\
45 \\
46 \\
45\end{array}$ & $\begin{array}{l}48 . \\
53 . \\
45 . \\
25 . \\
47 .\end{array}$ & $\begin{array}{l}636 . \\
564 . \\
734 . \\
609 . \\
556 .\end{array}$ \\
\hline $\begin{array}{l}147 \\
148 \\
149 \\
150 \\
151\end{array}$ & $\begin{array}{l}5 \\
5 \\
5 \\
5 \\
5\end{array}$ & $\begin{array}{l}27 \\
28 \\
29 \\
30 \\
31\end{array}$ & $\begin{array}{l}78 \\
78 \\
78 \\
78 \\
78\end{array}$ & $\begin{array}{r}.11 \\
.03 \\
0.00 \\
0.00 \\
0.00\end{array}$ & $\begin{array}{r}49^{\circ} \\
91^{\circ} \\
107^{\circ} \\
159^{\circ} \\
120^{\circ}\end{array}$ & $\begin{array}{l}40 \\
40 \\
43 \\
50 \\
44\end{array}$ & $\begin{array}{l}74 . \\
61 . \\
50 . \\
30 . \\
72 .\end{array}$ & $\begin{array}{l}379 . \\
528 . \\
707 . \\
626 . \\
651 .\end{array}$ \\
\hline $\begin{array}{l}152 \\
153 \\
154 \\
155 \\
156\end{array}$ & $\begin{array}{l}6 \\
6 \\
6 \\
6 \\
6\end{array}$ & $\begin{array}{l}1 \\
2 \\
3 \\
4 \\
5\end{array}$ & $\begin{array}{l}78 \\
78 \\
78 \\
78 \\
78\end{array}$ & $\begin{array}{l}0.00 \\
0.00 \\
0.00 \\
0.00 \\
.55\end{array}$ & $\begin{array}{l}89 . \\
67 . \\
92 . \\
750^{\circ} \\
43 .\end{array}$ & $\begin{array}{l}44 . \\
46 . \\
49 . \\
41 . \\
38\end{array}$ & $\begin{array}{l}82 . \\
73 . \\
67 . \\
97 . \\
94 .\end{array}$ & $\begin{array}{l}539 . \\
452 . \\
480 . \\
222 . \\
377 .\end{array}$ \\
\hline $\begin{array}{l}157 \\
158 \\
159 \\
160 \\
161\end{array}$ & $\begin{array}{l}6 \\
6 \\
6 \\
6 \\
6\end{array}$ & $\begin{array}{r}6 \\
7 \\
8 \\
9 \\
10\end{array}$ & $\begin{array}{l}78 \\
78 \\
78 \\
78 \\
78\end{array}$ & $\begin{array}{r}.23 \\
0.00 \\
.10 \\
0.00 \\
0.00\end{array}$ & $\begin{array}{r}97 . \\
93^{\circ} \\
130^{\circ} \\
146^{\circ} \\
33^{\circ}\end{array}$ & $\begin{array}{l}42 . \\
45 \\
47 \\
54 \\
58\end{array}$ & $\begin{array}{l}62 . \\
770^{\circ} \\
580^{\circ} \\
48 . \\
33 .\end{array}$ & $\begin{array}{l}696 . \\
482 . \\
696 . \\
719 . \\
631 .\end{array}$ \\
\hline $\begin{array}{l}162 \\
163 \\
164 \\
165 \\
166\end{array}$ & $\begin{array}{l}6 \\
6 \\
6 \\
6 \\
6\end{array}$ & $\begin{array}{l}11 \\
12 \\
13 \\
14 \\
15\end{array}$ & $\begin{array}{l}78 \\
78 \\
78 \\
78 \\
78\end{array}$ & $\begin{array}{l}0.00 \\
0.00 \\
0.00 \\
0.00 \\
0.00\end{array}$ & $\begin{array}{r}35 . \\
121 . \\
70 \\
110 \\
134 .\end{array}$ & $\begin{array}{l}56 . \\
53 \\
55 \\
55 \\
59\end{array}$ & $\begin{array}{l}35 . \\
66 . \\
50 . \\
40 . \\
34 .\end{array}$ & $\begin{array}{l}716 . \\
527 \\
600 \\
657 . \\
732 .\end{array}$ \\
\hline
\end{tabular}


Table 7.--Climatic data, Garo station, May-September 1977 and 1978--Continued

\begin{tabular}{|c|c|c|c|c|c|c|c|c|}
\hline $\begin{array}{r}\text { DAY } \\
\text { OF } \\
\text { YEAR }\end{array}$ & MONTH & DAY & YEAR & $\begin{array}{l}\text { PRECIP } \\
\text { (IN.) }\end{array}$ & $\begin{array}{l}\text { WIND } \\
\text { (MI./DAY) }\end{array}$ & $\begin{array}{l}\text { DAIL } \\
\text { TEMP } \\
\text { (F) }\end{array}$ & $\begin{array}{l}\text { Y AVG } \\
\text { REL HUM } \\
\text { (PERCENT) }\end{array}$ & $\begin{array}{l}\text { SOLAR } \\
\text { RAD } \\
\left(L Y_{.}\right)\end{array}$ \\
\hline $\begin{array}{l}167 \\
168 \\
169 \\
170 \\
171\end{array}$ & $\begin{array}{l}6 \\
6 \\
6 \\
6 \\
6\end{array}$ & $\begin{array}{l}16 \\
17 \\
18 \\
19 \\
20\end{array}$ & $\begin{array}{l}78 \\
78 \\
78 \\
78 \\
78\end{array}$ & $\begin{array}{l}0.00 \\
0.00 \\
0.00 \\
0.00 \\
0.00\end{array}$ & $\begin{array}{r}111 . \\
191 . \\
41 . \\
115 . \\
78 .\end{array}$ & $\begin{array}{l}57 . \\
51 \\
53 \\
56 \\
53 .\end{array}$ & $\begin{array}{l}260^{\circ} \\
30^{\circ} \\
49^{\circ} \\
44^{\circ} \\
54^{\circ}\end{array}$ & $\begin{array}{l}755 . \\
740 . \\
626 . \\
683 . \\
708 .\end{array}$ \\
\hline $\begin{array}{l}172 \\
173 \\
174 \\
175 \\
176\end{array}$ & $\begin{array}{l}6 \\
6 \\
6 \\
6 \\
6\end{array}$ & $\begin{array}{l}21 \\
22 \\
23 \\
24 \\
25\end{array}$ & $\begin{array}{l}78 \\
78 \\
78 \\
78 \\
78\end{array}$ & $\begin{array}{l}0.00 \\
0.00 \\
0.00 \\
0.00 \\
0.00\end{array}$ & $\begin{array}{r}140 \\
20 \\
63 \\
102 \\
121\end{array}$ & $\begin{array}{l}56 . \\
57 \\
61 \\
62 \\
61\end{array}$ & $\begin{array}{l}54{ }^{\circ} \\
430^{\circ} \\
430^{\circ} \\
40 . \\
36 .\end{array}$ & $\begin{array}{l}675 . \\
582 . \\
649 . \\
593 . \\
616 .\end{array}$ \\
\hline $\begin{array}{l}177 \\
178 \\
179 \\
180 \\
181\end{array}$ & $\begin{array}{l}6 \\
6 \\
6 \\
6 \\
6\end{array}$ & $\begin{array}{l}26 \\
27 \\
28 \\
29 \\
30\end{array}$ & $\begin{array}{l}78 \\
78 \\
78 \\
78 \\
78\end{array}$ & $\begin{array}{r}0.00 \\
0.00 \\
.09 \\
0.00 \\
.12\end{array}$ & $\begin{array}{l}97 . \\
47 . \\
51 . \\
67 .\end{array}$ & $\begin{array}{l}53 \\
53 \\
57 \\
55 \\
55\end{array}$ & $\begin{array}{l}42 . \\
73 \circ \\
61 . \\
81 . \\
67 .\end{array}$ & $\begin{array}{l}639 . \\
491 . \\
376 . \\
361 . \\
517 .\end{array}$ \\
\hline $\begin{array}{l}182 \\
183 \\
184 \\
185 \\
186\end{array}$ & $\begin{array}{l}7 \\
7 \\
7 \\
7 \\
7\end{array}$ & $\begin{array}{l}1 \\
2 \\
3 \\
4 \\
5\end{array}$ & $\begin{array}{l}78 \\
78 \\
78 \\
78 \\
78\end{array}$ & $\begin{array}{l}0.00 \\
0.00 \\
0.00 \\
0.00 \\
0.00\end{array}$ & $\begin{array}{r}92 . \\
58 . \\
23 \\
125 . \\
84 .\end{array}$ & $\begin{array}{l}55 \\
56 \\
58 \\
56 \\
56\end{array}$ & $\begin{array}{l}57 . \\
49 . \\
32 . \\
32 . \\
41 .\end{array}$ & $\begin{array}{l}494 . \\
556 . \\
600 . \\
749 . \\
619^{\circ}\end{array}$ \\
\hline $\begin{array}{l}187 \\
188 \\
189 \\
190 \\
191\end{array}$ & $\begin{array}{l}7 \\
7 \\
7 \\
7 \\
7\end{array}$ & $\begin{array}{r}6 \\
7 \\
8 \\
9 \\
10\end{array}$ & $\begin{array}{l}78 \\
78 \\
78 \\
78 \\
78\end{array}$ & $\begin{array}{l}0.00 \\
0.00 \\
0.00 \\
0.00 \\
0.00\end{array}$ & $\begin{array}{r}96 . \\
37 . \\
116 . \\
89 . \\
78 .\end{array}$ & $\begin{array}{l}54 \\
58 \\
60 \\
57 \\
54\end{array}$ & $\begin{array}{l}50 . \\
560^{\circ} \\
470^{\circ} \\
550^{\circ} \\
73 .\end{array}$ & $\begin{array}{l}687^{\circ} \\
664{ }^{\circ} \\
560^{\circ} \\
356 . \\
370^{\circ}\end{array}$ \\
\hline
\end{tabular}


Table 7.--Climatic data, Garo station, May-September 1977 and 1978--Continued

\begin{tabular}{|c|c|c|c|c|c|c|c|c|}
\hline $\begin{array}{r}\text { DAY } \\
\text { OF } \\
\text { YEAR }\end{array}$ & MONTH & DAY & YEAR & $\begin{array}{l}\text { PREC IF } \\
\text { (IN.) }\end{array}$ & $\begin{array}{c}\text { WIND } \\
\text { (MI./DAY) }\end{array}$ & $\begin{array}{r}\text { DAI } \\
\text { TEMP } \\
\text { (F) }\end{array}$ & $\begin{array}{l}\text { Y AVG } \\
\text { REL HUM } \\
\text { (PERCENT) }\end{array}$ & $\begin{array}{l}\text { SOLAR } \\
\text { RAD } \\
\text { (LY.) }\end{array}$ \\
\hline $\begin{array}{l}192 \\
193 \\
194 \\
195 \\
196\end{array}$ & $\begin{array}{l}7 \\
7 \\
7 \\
7 \\
7\end{array}$ & $\begin{array}{l}11 \\
12 \\
13 \\
14 \\
15\end{array}$ & $\begin{array}{l}78 \\
78 \\
78 \\
78 \\
78\end{array}$ & $\begin{array}{l}0.00 \\
0.00 \\
0.00 \\
0.00 \\
0.00\end{array}$ & $\begin{array}{l}60 . \\
44 . \\
530^{\circ} \\
60 . \\
67 .\end{array}$ & $\begin{array}{l}55 . \\
59 . \\
61 . \\
64 . \\
56 .\end{array}$ & $\begin{array}{l}66 . \\
62 . \\
57 . \\
50 . \\
65 .\end{array}$ & $\begin{array}{l}423 . \\
4670^{\circ} \\
5030^{\circ} \\
5130^{\circ} \\
240 .\end{array}$ \\
\hline $\begin{array}{l}197 \\
198 \\
199 \\
200 \\
201\end{array}$ & $\begin{array}{l}7 \\
7 \\
7 \\
7 \\
7\end{array}$ & $\begin{array}{l}16 \\
17 \\
18 \\
19 \\
20\end{array}$ & $\begin{array}{l}78 \\
78 \\
78 \\
78 \\
78\end{array}$ & $\begin{array}{l}0.00 \\
0.00 \\
.04 \\
0.00 \\
0.00\end{array}$ & $\begin{array}{l}62 . \\
950^{\circ} \\
50 \\
67 . \\
60 .\end{array}$ & $\begin{array}{l}55 . \\
52 . \\
67 . \\
59 . \\
55 .\end{array}$ & $\begin{array}{l}81 . \\
86 . \\
37 . \\
59 . \\
76 .\end{array}$ & $\begin{array}{l}323 . \\
503 . \\
588 . \\
431 . \\
446 .\end{array}$ \\
\hline $\begin{array}{l}202 \\
203 \\
204 \\
205 \\
206\end{array}$ & $\begin{array}{l}7 \\
7 \\
7 \\
7 \\
7\end{array}$ & $\begin{array}{l}21 \\
22 \\
23 \\
24 \\
25\end{array}$ & $\begin{array}{l}78 \\
78 \\
78 \\
78 \\
78\end{array}$ & $\begin{array}{r}.01 \\
0.00 \\
0.00 \\
.01 \\
0.00\end{array}$ & $\begin{array}{l}84 . \\
65 \\
51 . \\
57 \\
108\end{array}$ & $\begin{array}{l}58 . \\
57 . \\
58 . \\
58 . \\
60 .\end{array}$ & $\begin{array}{l}71 . \\
66 . \\
63 . \\
54 . \\
51 .\end{array}$ & $\begin{array}{l}497 . \\
599 . \\
556 . \\
534 . \\
550 .\end{array}$ \\
\hline $\begin{array}{l}207 \\
208 \\
209 \\
210 \\
211\end{array}$ & $\begin{array}{l}7 \\
7 \\
7 \\
7 \\
7\end{array}$ & $\begin{array}{l}26 \\
27 \\
28 \\
29 \\
30\end{array}$ & $\begin{array}{l}78 \\
78 \\
78 \\
78 \\
78\end{array}$ & $\begin{array}{r}0.00 \\
.17 \\
.01 \\
0.00 \\
.01\end{array}$ & $\begin{array}{r}44 . \\
39 . \\
119^{\circ} \\
23 . \\
59\end{array}$ & $\begin{array}{l}59 . \\
60 . \\
60 . \\
56 . \\
58 .\end{array}$ & $\begin{array}{l}60 . \\
58 . \\
62 . \\
79 . \\
67 .\end{array}$ & $\begin{array}{l}516 . \\
449 . \\
370 . \\
462 . \\
501 .\end{array}$ \\
\hline $\begin{array}{l}212 \\
213 \\
214 \\
215 \\
216\end{array}$ & $\begin{array}{l}7 \\
8 \\
8 \\
8 \\
8\end{array}$ & $\begin{array}{r}31 \\
1 \\
2 \\
3 \\
4\end{array}$ & $\begin{array}{l}78 \\
78 \\
78 \\
78 \\
78\end{array}$ & $\begin{array}{r}.19 \\
.01 \\
.05 \\
0.00 \\
.08\end{array}$ & $\begin{array}{r}4 * \\
107 \\
35 \\
80 \\
15 .\end{array}$ & $\begin{array}{r}4 \\
52 . \\
53 . \\
50 \\
53 .\end{array}$ & $\begin{array}{r}\$ 4 \% \\
74 . \\
76 . \\
87 . \\
71 .\end{array}$ & $\begin{array}{l}346 . \\
423 . \\
540 \\
366 . \\
488 .\end{array}$ \\
\hline
\end{tabular}


Table 7.--Climatic data, Garo station, May-September 1977 and 1978--Continued

\begin{tabular}{|c|c|c|c|c|c|c|c|c|}
\hline $\begin{array}{r}\text { DAY } \\
\text { OF } \\
\text { YEAR }\end{array}$ & MONTH & DAY & YEAR & $\begin{array}{l}\text { PRECIF } \\
(I N .)\end{array}$ & $\begin{array}{c}\text { WINU } \\
\text { (MI./DAY) }\end{array}$ & $\begin{array}{l}\text { DAII } \\
\text { TEMP } \\
\text { (F) }\end{array}$ & $\begin{array}{l}\text { Y AVG } \\
\text { REL HUM } \\
\text { (PERCENT) }\end{array}$ & $\begin{array}{l}\text { SOLAR } \\
\text { RAD } \\
(L Y .)\end{array}$ \\
\hline $\begin{array}{l}217 \\
218 \\
219 \\
220 \\
221\end{array}$ & $\begin{array}{l}8 \\
8 \\
8 \\
8 \\
8\end{array}$ & $\begin{array}{l}5 \\
6 \\
7 \\
8 \\
9\end{array}$ & $\begin{array}{l}78 \\
78 \\
78 \\
78 \\
78\end{array}$ & $\begin{array}{l}0.00 \\
0.00 \\
0.00 \\
0.00 \\
.01\end{array}$ & $\begin{array}{l}52 . \\
81 . \\
63{ }^{\circ} \\
54 . \\
47 .\end{array}$ & $\begin{array}{r}53 . \\
55 . \\
* 4 \\
53 . \\
* * *\end{array}$ & $\begin{array}{r}56 . \\
67 . \\
* * 4 \\
67 . \\
* * *\end{array}$ & $\begin{array}{l}625 . \\
387 . \\
441 . \\
340 . \\
488 .\end{array}$ \\
\hline $\begin{array}{l}222 \\
223 \\
224 \\
225 \\
226\end{array}$ & $\begin{array}{l}8 \\
8 \\
8 \\
8 \\
8\end{array}$ & $\begin{array}{l}10 \\
11 \\
12 \\
13 \\
14\end{array}$ & $\begin{array}{l}78 \\
78 \\
78 \\
78 \\
78\end{array}$ & $\begin{array}{r}.14 \\
.04 \\
.06 \\
.11 \\
0.00\end{array}$ & $\begin{array}{r}81 \\
45 \\
75 \\
24 \\
24 \\
126\end{array}$ & $\begin{array}{l}4 * 4 \\
54 . \\
55 . \\
53 . \\
44 .\end{array}$ & $\begin{array}{r}* * * \\
75 . \\
72 . \\
74 . \\
67 .\end{array}$ & $\begin{array}{l}289^{\circ} \\
360 . \\
376 . \\
462 . \\
439 .\end{array}$ \\
\hline $\begin{array}{l}227 \\
228 \\
229 \\
230 \\
231\end{array}$ & $\begin{array}{l}8 \\
8 \\
8 \\
8 \\
8\end{array}$ & $\begin{array}{l}15 \\
16 \\
17 \\
18 \\
19\end{array}$ & $\begin{array}{l}78 \\
78 \\
78 \\
78 \\
78\end{array}$ & $\begin{array}{l}.10 \\
0.00 \\
0.00 \\
0.00 \\
0.00\end{array}$ & $\begin{array}{r}133 . \\
64 \\
25 \\
111^{\circ} \\
54\end{array}$ & $\begin{array}{l}49 . \\
54 . \\
56 . \\
49 . \\
47 .\end{array}$ & $\begin{array}{l}47 . \\
460^{\circ} \\
39^{\circ} \\
600^{\circ} \\
69^{\circ}\end{array}$ & $\begin{array}{l}631 . \\
651 . \\
624 . \\
513 . \\
444 .\end{array}$ \\
\hline $\begin{array}{l}232 \\
233 \\
234 \\
235 \\
236\end{array}$ & $\begin{array}{l}8 \\
8 \\
8 \\
8 \\
8\end{array}$ & $\begin{array}{l}20 \\
21 \\
22 \\
23 \\
24\end{array}$ & $\begin{array}{l}78 \\
78 \\
78 \\
78 \\
78\end{array}$ & $\begin{array}{l}0.00 \\
0.00 \\
0.00 \\
0.00 \\
0.00\end{array}$ & $\begin{array}{l}67 . \\
41 . \\
770 \\
770 \\
23 .\end{array}$ & $\begin{array}{l}53 . \\
54 . \\
55 . \\
53 . \\
53 .\end{array}$ & $\begin{array}{l}63 . \\
62 . \\
76 . \\
67 . \\
69 .\end{array}$ & $\begin{array}{l}500 . \\
369 . \\
359 . \\
439 . \\
292 .\end{array}$ \\
\hline $\begin{array}{l}237 \\
238 \\
239 \\
240 \\
241\end{array}$ & $\begin{array}{l}8 \\
8 \\
8 \\
8 \\
8\end{array}$ & $\begin{array}{l}25 \\
26 \\
27 \\
28 \\
29\end{array}$ & $\begin{array}{l}78 \\
78 \\
78 \\
78 \\
78\end{array}$ & $\begin{array}{r}0.00 \\
.10 \\
0.00 \\
0.00 \\
.21\end{array}$ & $\begin{array}{l}79 . \\
750^{\circ} \\
45 . \\
64 . \\
56 .\end{array}$ & $\begin{array}{l}54 . \\
53 . \\
56 . \\
46 . \\
48 .\end{array}$ & $\begin{array}{l}85 . \\
62 . \\
48 . \\
71 . \\
84 .\end{array}$ & $\begin{array}{l}341 . \\
484{ }^{\circ} \\
484 . \\
441 . \\
389 .\end{array}$ \\
\hline 242 & 8 & 30 & 78 & 0.00 & 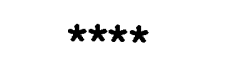 & 49 & 74. & 38 \\
\hline
\end{tabular}


$\cdots$

. 
